In cooperation with the Texas Department of Transportation

\title{
Effects of Regulation on L-moments of Annual Peak Streamflow in Texas
}

Water-Resources Investigations Report 01-4243

U.S. Department of the Interior

U.S. Geological Survey 
Technical Report Documentation Page

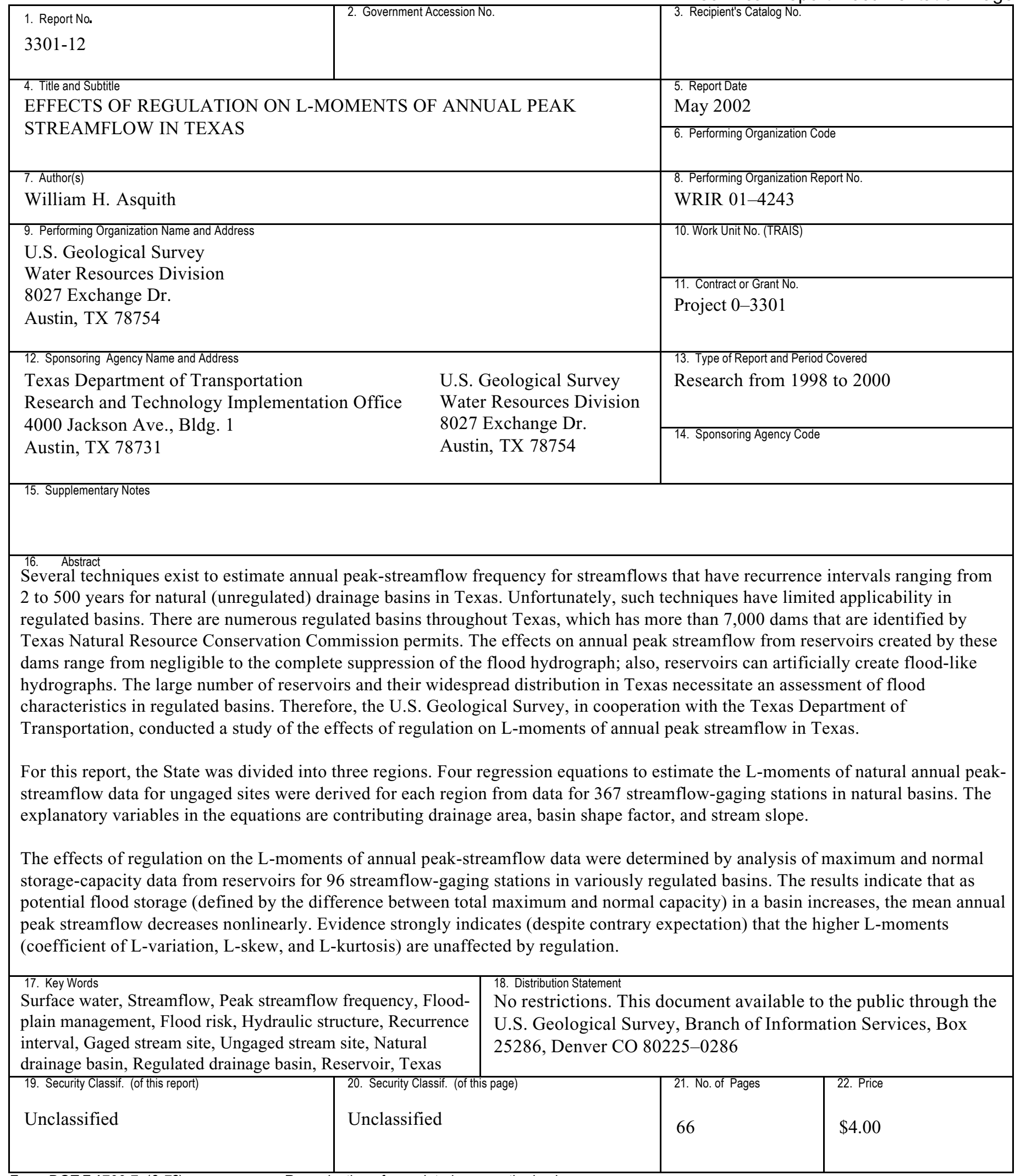

Form DOTF 1700.7 (8-72)

Reproduction of completed page authorized

Cover: Medina Dam and Medina Lake, August 1994 (photograph by Ted A. Small, U.S. Geological Survey). 
U.S. Department of the Interior

U.S. Geological Survey

\section{Effects of Regulation on L-moments of Annual Peak Streamflow in Texas}

By William H. Asquith

\section{U.S. GEOLOGICAL SURVEY}

Water-Resources Investigations Report 01-4243

In cooperation with the Texas Department of Transportation

Austin, Texas

2001 


\section{U.S. DEPARTMENT OF THE INTERIOR}

Gale A. Norton, Secretary

\section{U.S. GEOLOGICAL SURVEY}

Charles G. Groat, Director

Any use of trade, product, or firm names is for descriptive purposes only and does not imply endorsement by the U.S. Government.

For additional information write to

\section{District Chief}

U.S. Geological Survey

8027 Exchange Dr.

Austin, TX 78754-4733

E-mail: dc_tx@usgs.gov

Copies of this report can be purchased from

U.S. Geological Survey

Branch of Information Services

Box 25286

Denver, CO 80225-0286

E-mail: infoservices@usgs.gov 


\section{CONTENTS}

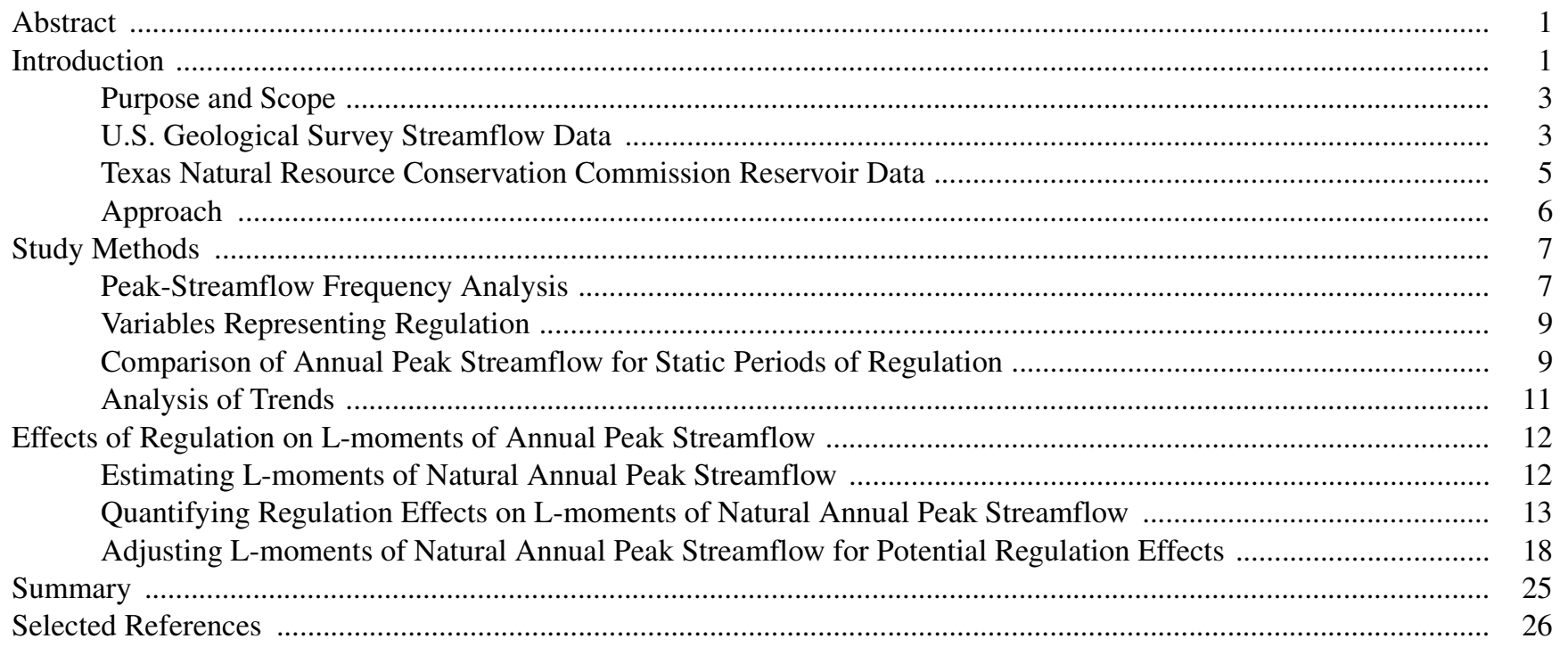

\section{PLATES}

(Plates are in pocket)

1-2. Maps showing:

1. Location of U.S. Geological Survey streamflow-gaging stations used in analysis

2. Location of dams in Texas Natural Resource Conservation Commission inventory of dams used in analysis

\section{FIGURES}

1-2. Maps showing:

1. Location of 367 streamflow-gaging stations with at least 10 years of annual peak-streamflow data through 1997 (designated as natural by 10-percent criterion)

2. Location of 96 streamflow-gaging stations with at least 10 years of annual peak-streamflow data through 1997 (designated as regulated by 10-percent criterion) that have static periods of regulation suitable for comparative analysis

3-13. Graphs showing:

3. Example of time series of cumulative changes in regulation for station 08136500 Concho River at Paint Rock, Texas

4. Relation between (a) change in maximum capacity, (b) change in normal capacity, and (c) change in difference between maximum and normal capacity to contributing drainage area in all regions

5. Relation between (a) change in maximum capacity, (b) change in normal capacity, and (c) change in difference between maximum and normal capacity to contributing drainage area in northeast region

6. Relation between (a) change in maximum capacity, (b) change in normal capacity, and (c) change in difference between maximum and normal capacity to contributing drainage area in southeast region

7. Relation between (a) change in maximum capacity, (b) change in normal capacity, and (c) change in difference between maximum and normal capacity to contributing drainage area in west region .......

8. Relation between change in mean annual peak streamflow to (a) change in maximum capacity and (b) change in normal capacity 
9. Relation between change in L-CV of annual peak streamflow to (a) change in maximum capacity and (b) change in normal capacity

10. Relation between change in L-skew of annual peak streamflow to (a) change in maximum capacity and (b) change in normal capacity

11. Relation between (a) change in mean annual peak streamflow and (b) change in L-CV of annual peak streamflow to change in difference between maximum and normal capacity

12. Relation between change in L-skew of annual peak streamflow and change in difference between maximum and normal capacity

13. Relation between (a) change in mean annual peak streamflow and (b) change in L-CV of annual peak streamflow to contributing drainage area

\section{TABLES}

1. Streamflow statistics and basin characteristics for 367 USGS streamflow-gaging stations with at least 10 years of annual peak-streamflow data through 1997 (designated as natural by 10-percent criterion)

2. Static periods of regulation suitable for comparative analysis for 96 USGS streamflow-gaging stations with at least 10 years of annual peak-streamflow data through 1997 (designated as regulated by 10percent criterion)

3. Texas Natural Resource Conservation Commission permitted dams with maximum capacities in excess of 10,000 acre-feet

4. Regression equations for estimation of L-moments and L-moment ratios of annual peak streamflow for three regions in Texas 


\title{
Effects of Regulation on L-moments of Annual Peak Streamflow in Texas
}

\author{
By William H. Asquith
}

\section{Abstract}

Several techniques exist to estimate annual peak-streamflow frequency for streamflows that have recurrence intervals ranging from 2 to 500 years for natural (unregulated) drainage basins in Texas. Unfortunately, such techniques have limited applicability in regulated basins. There are numerous regulated basins throughout Texas, which has more than 7,000 dams that are identified by Texas Natural Resource Conservation Commission permits. The effects on annual peak streamflow from reservoirs created by these dams range from negligible to the complete suppression of the flood hydrograph; also, reservoirs can artificially create flood-like hydrographs. The large number of reservoirs and their widespread distribution in Texas necessitate an assessment of flood characteristics in regulated basins. Therefore, the U.S. Geological Survey, in cooperation with the Texas Department of Transportation, conducted a study of the effects of regulation on L-moments of annual peak streamflow in Texas.

For this report, the State was divided into three regions. Four regression equations to estimate the L-moments of natural annual peak-streamflow data for ungaged sites were derived for each region from data for 367 streamflow-gaging stations in natural basins. The explanatory variables in the equations are contributing drainage area, basin shape factor, and stream slope.

The effects of regulation on the L-moments of annual peak-streamflow data were determined by analysis of maximum and normal storagecapacity data from reservoirs for 96 streamflowgaging stations in variously regulated basins. The results indicate that as potential flood storage (defined by the difference between total maximum and normal capacity) in a basin increases, the mean annual peak streamflow decreases nonlinearly. Evidence strongly indicates (despite contrary expectation) that the higher L-moments (coefficient of L-variation, L-skew, and L-kurtosis) are unaffected by regulation.

\section{INTRODUCTION}

Estimates of peak-streamflow frequency are useful for flood-plain management, for objective assessment of flood risk, and for cost-effective design of hydraulic structures. Peak-streamflow frequency refers to peak streamflows that have recurrence intervals of 2, $5,10,25,50,100,250$, and 500 years. Several techniques exist to estimate peak-streamflow frequency for gaged and ungaged stream sites in natural (unregulated) watersheds (basins) in Texas (Asquith and Slade, 1997, 1999). Although such techniques are sometimes applicable for gaged sites, the techniques are of questionable applicability for ungaged stream sites whose watersheds are affected by human-produced regulation, principally through the construction of dams. To address this situation, the U.S. Geological Survey (USGS), in cooperation with the Texas Department of Transportation (TxDOT), in 1998 began an investigation of flood characteristics as expressed by the L-moments of annual peak streamflow in regulated basins in Texas.

Historically, a natural basin in Texas has been defined by the USGS as a basin with less than 10percent impervious cover, less than 10 percent of its drainage area controlled by reservoirs, and no other human-related factors that would affect peak streamflow. Impervious cover is not a consideration for this report. At present (2001), the degree of actual control (regulation) in a given part of a basin is assessed by USGS personnel on a more qualitative than quantitative basis. A regulated basin thus was initially identified for this investigation as a basin for which 10 percent or more of the drainage area of the basin is affected or controlled by reservoirs. In some other states the USGS uses a 10-percent change in the annual peak streamflow 
to define a regulated basin. Benson $(1962,1964)$ determined that about 100 acre-feet per square mile $\left(\right.$ acre- $\mathrm{ft} / \mathrm{mi}^{2}$ ) of flood storage in the drainage area reduces the annual peak streamflow by about 10 percent in humid areas, and that about $50 \mathrm{acre}-\mathrm{ft} / \mathrm{mi}^{2}$ of flood storage reduces the annual peak streamflow by about 10 percent in arid areas.

The criterion of 10 percent or more of the drainage area controlled by reservoirs was used as a starting point for the investigation. Such a simple classification scheme, though easy to implement in practice, is fraught with problems. Regulation in reality encompasses a wide spectrum of human influences on annual peak streamflow. For example, a specific reservoir or suite of reservoirs might dramatically alter low flows and midrange flows but leave higher flows, such as the annual peaks, largely unaffected. Appreciable effort was spent during the course of the investigation to scientifically and statistically differentiate between the various degrees of regulation on annual peak streamflow in a given basin without regard to how regulation might influence other flows. In this report, "regulated" and its variants refer to the entire spectrum of human influence on peak streamflow, and "natural" refers to the unregulated end-member of the regulation spectrum. It is unknown whether the unregulated end-member fully applies for basins considered in this investigation because of widespread modification of watershed hydrology by dams throughout Texas.

To clarify, the popular binary designation of a watershed as either natural or regulated is not sufficient to accurately express the degree of regulation because of the presence of complex arrangements of flowaltering structures in the majority of basins across the entire range of drainage areas in Texas. Such structures include contour-plowing of agricultural lands for erosion control and enhanced water retention, bulldozed earthen embankments on the smallest of usually dry tributaries forming "Texas stock tanks," low-head dams built nearly as high as the tops of stream banks, small floodwater-retarding structures, constant-level recreational and water-supply reservoirs, and colossal flood-control reservoirs on many of the rivers in Texas.

The USGS, in cooperation with TxDOT, has comprehensively researched peak-streamflow frequency for streams having natural basins defined by the 10-percent controlled drainage area criterion in Texas (Asquith and Slade, 1995, 1997, 1999; Judd and others, 1996; and Slade and Asquith, 1996). During these natural peak-streamflow investigations, procedures were developed to estimate the 2-, 5-, 10-, 25-, 50-, and 100-year recurrence interval peak streamflow. Two dominant limitations of the natural peak-streamflow investigations are: (1) The USGS streamflow-gaging network in Texas lacks sufficient representation for small drainage basins (less than about 50 square miles $\left[\mathrm{mi}^{2}\right]$ ), resulting in considerable potential error in peak-streamflow frequency estimates for small basins; and (2) many of the moderate-tolarge watersheds in Texas (greater than about $200 \mathrm{mi}^{2}$ ) are controlled or regulated to variable extents by dams, flood-detention structures, and flood-retention structures. Therefore, the peak-streamflow data from many stations were excluded from the natural peakstreamflow investigations, and the applicability of resulting peak-streamflow frequency estimation procedures to natural basins is limited.

To address the first limitation of the investigations, the USGS, in cooperation with TxDOT, comprehensively researched heavy precipitation in Texas, including the depths for various durations and recurrence intervals or recurrence periods (Asquith, 1998a, 1999; Asquith and Famiglietti, 2000; and Lanning-Rush and others, 1998). Precipitation characteristics can be used, with appropriate statistical or simulation models, to estimate peak-streamflow characteristics for streams in small watersheds. The investigation of flooding characteristics for regulated basins in Texas presented in this report addresses the second limitation of the natural peak-streamflow investigations.

Peak streamflow in regulated basins is affected by runoff from the unregulated part of the basin and by runoff from the regulated part of the basin. The regulated runoff is affected by discharges from dams or reservoirs and by the quantity, type, and spatial distribution of flood-detention or flood-retention structures, commonly termed "small floodwater-retarding structures." Small floodwater-retarding structures is a broad term and includes all identified small-to-moderate-size dams in Texas whether or not the original purpose for their construction was flood control. Peak streamflows from reservoirs represent controlled discharges (releases) and uncontrolled discharges through pipes, spillways, or other structures. Controlled releases often are dictated by flood-management practices and water-supply concerns, which might have opposing objectives. There are more than 200 major reservoirs in Texas-those having maximum storage capacities in excess of 10,000 acre-feet (acre-ft). This total also includes a few offchannel and diversion-oriented reservoirs. 
The characteristics of regulated basins are difficult to assess and incorporate into procedures for peakstreamflow frequency estimation because of the inherent difficulties in quantifying the aggregate influence of all controls in a given basin. The effect of reservoirs on downstream peak streamflow can range from negligible to the complete suppression of the flood hydrograph; also, reservoir releases can artificially create flood-like hydrographs. Reservoir influence varies for the same reservoir or for a suite of reservoirs because of factors including storage conditions at the time of a flood, management decisions (if applicable) regarding reservoir operation, and magnitude and timing of peaks from the unregulated part of the basin. Thorough consideration of these factors, often in conjunction with streamflowrouting models, is required for the most accurate estimates of peak-streamflow frequency. Investigations encompassing the variety of possible reservoir influences are expensive. Also such investigations would be limited to watersheds that have a sufficient record of unregulated peak streamflow upstream from or coincident with a reservoir site, definable operating rules for the reservoir, and repetitive management practices. In such cases, the unregulated record can be converted to regulated record using hydraulic routing. This method commonly is used by the U.S. Army Corps of Engineers and others. Unfortunately, few basins have all the required data. As a result, an alternative approach was developed that uses readily available data.

\section{Purpose and Scope}

The purpose of this report is to document, quantify, and predict effects of regulation on annual peak streamflow in Texas through analysis of the changes in the L-moment statistics of the annual peak streamflow. The data for the study were limited to the USGS peakstreamflow database in Texas and related basin characteristics and limited to the Texas Natural Resource Conservation Commission (TNRCC) inventory of dams. The inventory of dams was acquired by the USGS in September 1997.

\section{U.S. Geological Survey Streamflow Data}

Streamflow statistics from the USGS database of annual peak-streamflow data of natural basins (10percent or less controlled drainage area) are used in later sections of this report. The 367 stations in natural basins identified for this report have an average record length of about 30 years. The stations all have at least 10 or more years of natural annual peak-streamflow data through 1997, are part of the Texas streamflow-gaging network, and are a subset of the natural stations used by Asquith and Slade (1997). The 367-station subset was chosen because about 190 of the approximately 560 stations identified by Asquith and Slade (1997) have less than 10 years of data and typically are in very small watersheds (less than about $10 \mathrm{mi}^{2}$ ). These small watersheds have very short record, often have relatively more error in the basin characteristic estimates, and the stage-discharge relation for the station often is theoretical (R.M. Slade, Jr., U.S. Geological Survey, oral commun., 2000). One station, 07300500 Salt Fork Red River at Mangum, Okla. (not part of the Texas network), was included because it has more than 60 years of peakstreamflow data and is located near a part of Texas where data are sparse.

The locations of the natural stations are shown on plate 1 and in figure 1 . The stations are identified in table 1 (at end of report) along with pertinent streamflow statistics that are discussed in later sections of this report. Also shown on plate 1 and in figure 1 is a threeregion delineation of Texas. These regions (northeast, southeast, and west) were selected on the basis of regional boundaries used by Asquith and Slade (1995, 1997), consideration of reservoir density, and (most importantly) density and locations of regulated streamflow-gaging stations. The regions are used in analyses discussed in later sections of this report titled, "Estimating L-moments of Natural Annual Peak Streamflow" and "Quantifying Regulation Effects on L-moments of Natural Annual Peak Streamflow."

The USGS database of annual peak-streamflow data also contains about 330 stations on regulated streams with an average record length of about 26 years. "Regulated" at this point in the report continues to refer to the historical 10-percent controlled drainage area criterion. Numerous USGS stations have both natural and regulated record. About 192 stations have 10 or more years of regulated record through 1997 . The average period of record for these stations is about 28 years. However, only 96 stations, which are a subset of the 192 regulated stations, were suitable for study — details are provided in later sections of this report. The mean period of regulated record for the 96 regulated stations is about 34 years. The locations of the 96 regulated stations are shown on plate 1 and in figure 2 . These stations (listed in table 2, at end of report, along with pertinent streamflow statistics and discussed in later sections of this report) are located in every major river basin in 


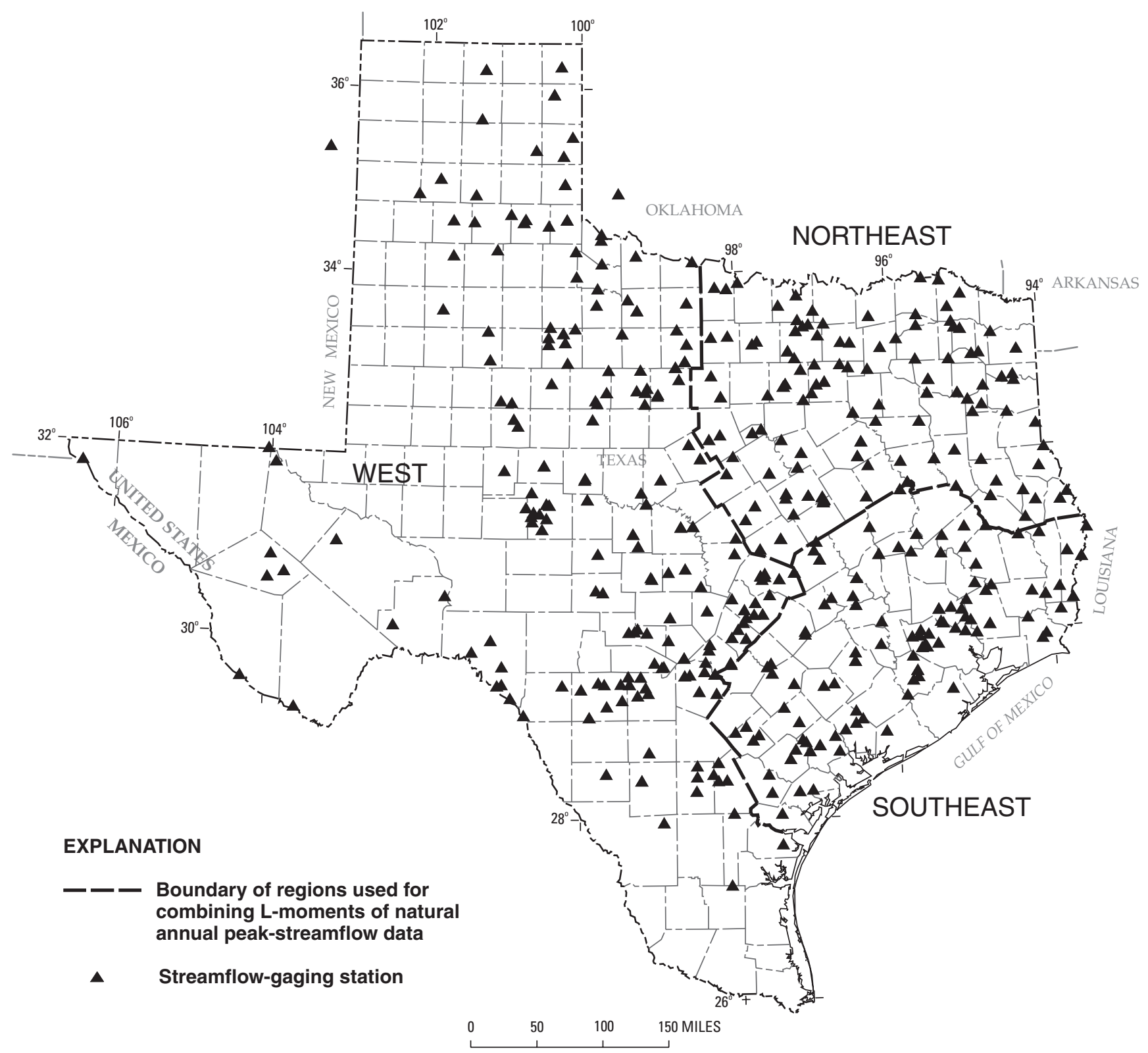

Figure 1. Location of 367 streamflow-gaging stations with at least 10 years of annual peak-streamflow data through 1997 (designated as natural by 10-percent criterion).

Texas and are distributed around the State. Because the data for these stations represent the combined influence or synthesis of the reservoir effects (regulation) and unmodified runoff from natural areas, the database can be used to assess regulated peak-streamflow frequency throughout much of the State.

Three physical basin characteristics were selected for the investigation: contributing drainage area, basin shape factor, and stream slope. The same basin characteristics were used by Asquith and Slade (1997). The contributing drainage area is expressed in square miles. The stream length for stream slope and basin shape factor computation is defined from the longest mapped channel from the station to the headwaters, on the basis of quadrangle maps prepared by the USGS (scale, 1:100,000). The basin shape factor is the ratio of the stream length to the contributing drainage area, which mathematically represents the square of the ratio of the longest stream length to the mean width of the basin. The basin shape factor is dimensionless. The stream 


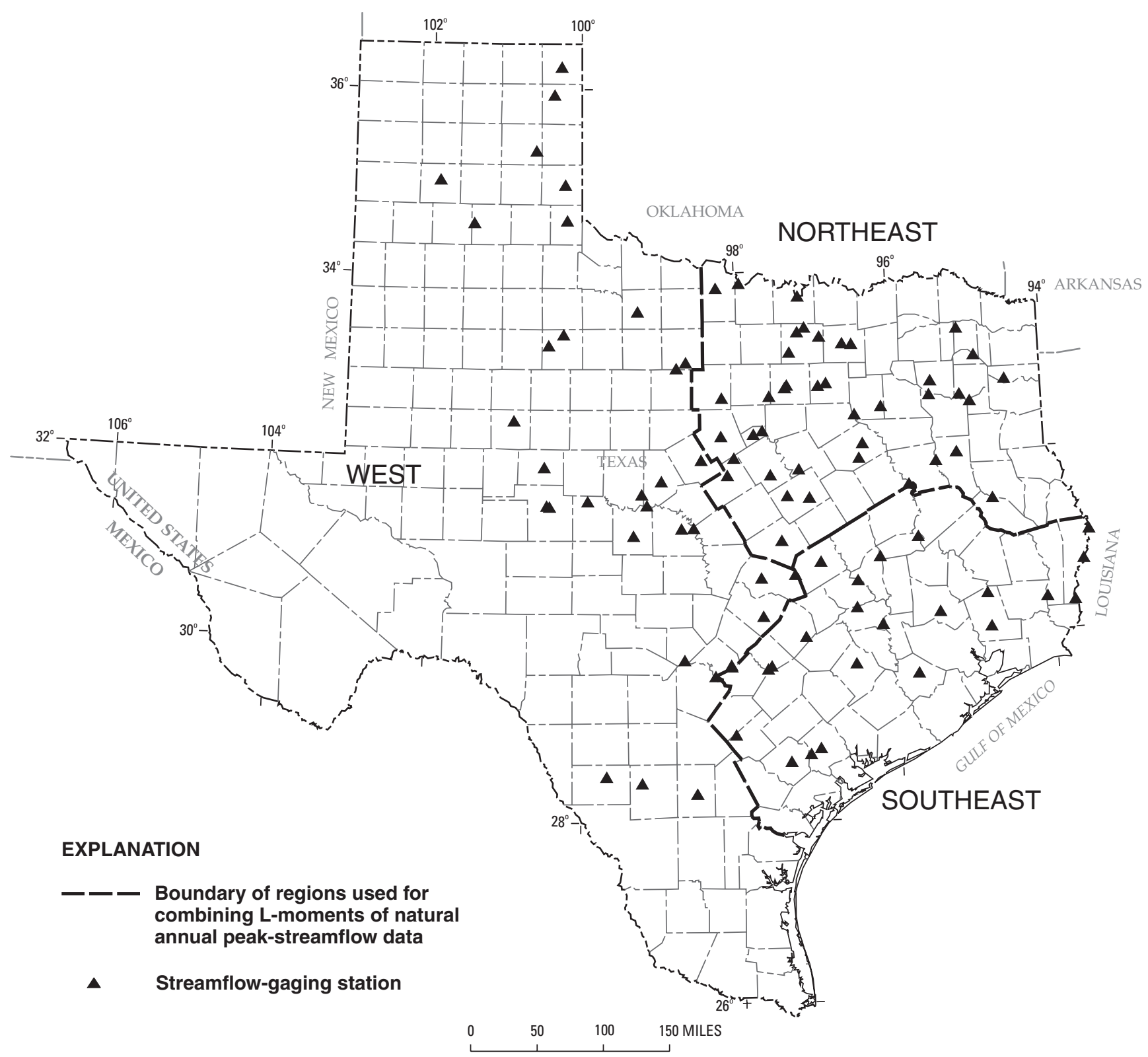

Figure 2. Location of 96 streamflow-gaging stations with at least 10 years of annual peak-streamflow data through 1997 (designated as regulated by 10-percent criterion) that have static periods of regulation suitable for comparative analysis.

slope, expressed in feet per mile, is the ratio of the difference in elevation of the longest mapped channel between the station and the headwaters to the length of the longest mapped channel.

\section{Texas Natural Resource Conservation Commission Inventory of Dams}

Another database pertinent to this investigation is maintained by the TNRCC; this database contains about
50 characteristics for each permitted dam in Texas. Some of the pertinent characteristics and the primary ones used for this report include:

Dam number-The TNRCC Dam Safety Team sevencharacter identification number.

Dam name-The official or most widely recognized name of the dam. The dam name is not necessarily the same as, or related to, the name of the impounded reservoir. These names can change with time. 
Reservoir name-The official or most widely recognized name of the reservoir.

Latitude and longitude - Latitude and longitude of the dam at its maximum section.

County-Name of the county where the dam is located.

Dam purpose-The purpose for which the reservoir is used. Some examples are water supply, domestic and livestock, irrigation, recreation, flood control, and fish and wildlife. For purposes of this study only dams having a flood-control purpose are separately identified; all others are lumped as nonflood control.

Year completed-The year that the original main dam structure was completed, which can have little relation to the year that the reservoir was initially filled.

Maximum capacity - The maximum capacity of the reservoir, which is defined as the total storage space in a reservoir below the maximum attainable watersurface elevation, rounded to the nearest whole acrefoot.

Normal capacity - The normal capacity of the reservoir, which is defined as the total storage space in a reservoir below the normal retention level or the lowest ungaged/ungated outlet (normal water level), rounded to the nearest whole acre-foot.

Surface area-The surface area of the reservoir at its normal retention level, in acres.

Drainage area-The contributing drainage area of the watershed upstream of the dam, in square miles.

The total number of dams in the inventory exceeds 7,300. Not all the data fields pertinent to this study were complete; for example latitude, longitude, and other important information might be missing. Excluding dams with extensive missing data resulted in 7,035 dams included in the database for this study. As part of the preparation of the dam database, it was determined that 17 of the 7,035 dams had incorrect latitude and longitude; these values were easily revised. The locations of the 7,035 dams are shown on plate 2; the dams are differentiated on the basis of maximum capacity (greater than or less than 10,000 acre-ft) and on whether flood control is one of the purposes for the dam.

A check to determine whether each of the 7,035 dams plotted on a map within its identified county indicated that only 59 dams were not in the identified county. Incorrectly located dams represent a very small fraction of the total number of dams, and associated capacities also were a very small fraction of the total storage and surface area values for the State. For example, the mean maximum capacity of the incorrectly located dams is 631 acre-ft-a very small amount. The locations of the 59 dams could not be easily corrected and were not corrected for this study.

For a small number of dams, the fields for maximum capacity and normal capacity are missing or zero. For a moderate number of dams, the surface area and drainage area are missing or zero. These missing values computationally were considered as zero-it was preferable to leave a dam in the analysis when at least one of either maximum or normal capacity, or surface area, was greater than zero. Of the 7,035 dams, 237 have missing maximum capacity, 113 have missing normal capacity, and 2,324 have missing surface area. The statewide total maximum capacity is about 98.6 million acre- $\mathrm{ft}$, total normal capacity is about 41.5 million acre$\mathrm{ft}$, and total surface area is about 1.25 million acres. The statewide totals are all underestimated because of missing data. The total surface area is the most underestimated. Reservoir surface area is not considered further in this report.

Finally, 217 dams have maximum capacities exceeding 10,000 acre-ft. These are referred to as "large" dams. Because this very small subset of dams has a disproportionate influence on streamflow, particular care was taken to verify the data fields. The large dams and their ancillary information are listed in table 3 (at end of report). A questionnaire was mailed to the owner/operator of each large dam in August 1998 to verify, update, or correct the information contained in the TNRCC inventory of dams. A summary of the results of the questionnaire is listed in table 3. The database for this study was revised, but the source TNRCC dam database was not revised using the revisions prior to the completion of this study.

\section{Approach}

The basic approach for the study is outlined below. Further details are provided throughout the remainder of the report.

\section{Estimate L-moments of natural annual peak} streamflow-A standard regional regression technique, following the model of Asquith and Slade (1997), was used to develop equations from the 367 streamflow-gaging stations in natural basins to estimate the L-moments of natural annual peak streamflow for ungaged sites. L-moment statistics are introduced later in the 
section titled "Peak-Streamflow Frequency Analysis." Separate equations were developed for the northeast, southeast, and west regions previously identified (pls. 1, 2; figs. 1, 2).

2. Quantify regulation effects on L-moments of annual peak streamflow - The effects of regulation on L-moments of natural annual peak streamflow were assessed by comparison of changes in L-moments to changes in regulation-that is, changes in upstream dam (reservoir) statistics-for 96 selected regulated streamflow-gaging stations. The changes in regulation were determined between consecutive static (unchanging) or acceptably static (minimally changing) time periods of dam influence.

3. Adjust L-moments of natural annual peak streamflow for regulation-Apply the results of step 2 to adjust L-moments estimated from step 1 for the estimated effects of regulation.

\section{STUDY METHODS}

This section outlines the four themes of analysis used. The themes are the statistical methods used to perform peak-streamflow frequency analysis, the variables representing regulation, the use of comparisons of annual peak streamflow for consecutive static or minimally changing periods of regulation, and the statistical method used in the analysis of trends in annual peak streamflow between static periods of regulation.

\section{Peak-Streamflow Frequency Analysis}

It has long been recognized that accurately estimating the magnitude and frequency of extreme hydrologic phenomena, such as peak-streamflow frequency, is difficult. The principal difficulty is that datasets generally are much shorter than the recurrence intervals (frequency) required by the objectives of a study. Quantile estimates (such as the 99-percent nonexceedance or 100-year flood) for large recurrence intervals commonly cannot be derived directly from the data. Thus, it is necessary to rely on a parametric probability distribution, which, when fitted to the data at the station, provides extrapolation to large recurrence intervals. A parametric probability distribution is exactly defined by a specified number of parameters- the number of parameters depends on the particular distribution. A probability distribution is fitted to the data by equating the moments of the data to the theoretical moments of a distribution, which in turn provides estimates of the parameters. The quantile accuracy of a particular distribution is directly related to the accuracy of the parameter estimates. Parameter accuracy in turn is directly related to the accuracy of the moment estimates. The identification of a suitable statistical distribution for estimating the peak-streamflow frequency is important. A comprehensive investigation of suitable distributions for Texas annual peak streamflow is beyond the scope of this report. However, some discussion of a candidate distribution is provided.

A fundamental element of a peak-streamflow frequency analysis is the statistical summarization of the recorded annual peak streamflow for a particular station. Traditionally, the peak-streamflow data have been statistically summarized by the product moments of the data (arithmetic mean, standard deviation or variance, skew, and kurtosis). The mathematical definitions of the product moments are widely known and require no formal definition here. However, these statistics are not always satisfactory for environmental datasets such as annual peak streamflow because the sample estimates of those statistics often exhibit large bias and sampling variance (Kirby, 1974; Wallis and others, 1974). To mitigate these problems, for this study, L-moment statistics, or L-moments, were used to summarize the data for each station (Stedinger and others, 1993). L-moments have many advantages over the product moments. Specifically, L-moments are less sensitive to the presence of outliers in the data, exhibit less bias, are more accurate in small samples, and do not require logarithmic transformation of the data for peakstreamflow frequency application. Hosking (1990) provides the definitive work on L-moment statistics. Hosking and Wallis (1997) provide the definitive work on application of L-moments for frequency analysis, including peak-streamflow frequency. L-moments are a relatively new development in the statistical sciences, thus a formal definition is provided here:

If $X_{1}, X_{2}, \ldots, X_{n}$ are $n$ independent and identically distributed continuous random variables having a cumulative distribution function $F(x)$, then the ordered values $X_{1: n} \leq X_{2: n} \leq \ldots \leq X_{n: n}$ are known as the order statistics (David, 1981) of the random sample $X_{1}, X_{2}$, $\ldots, X_{n}$. Clarification of the $X_{j: n}$ notation might be needed: $n$ represents the sample size, and $j$ represents the sequence order of a given sample when the entire sample is sorted in ascending order. The theoretical 
expectation of the $j$ th order statistic from a sample of size $n$ can be expressed in terms of the inverse cumulative distribution of $F(x), x(F)$, as:

$$
\begin{aligned}
E\left[X_{j: \mathrm{n}}\right]= & \frac{n !}{(j-1) !(n-j) !} \\
& \int_{0}^{1} x(F)^{j-1}(1-F)^{n-j} d F .
\end{aligned}
$$

In equation 1, the inverse cumulative distribution function is substituted for $x$. By taking linear combinations of the order statistic expectations, the L-moments are derived from the following equation:

$$
\lambda_{r+1}=\frac{1}{r+1} \sum_{k=0}^{r}(-1)^{k}\left(\begin{array}{l}
r \\
k
\end{array}\right) E\left[X_{(r-k+1):(r+1)}\right]
$$

From equation 2, the first four L-moments $\left(\lambda_{r}\right)$ are defined as:

$$
\begin{gathered}
\lambda_{1}=E\left[X_{1: 1}\right] ; \\
\lambda_{2}=\frac{1}{2}\left\{E\left[X_{2: 2}\right]-E\left[X_{1: 2}\right]\right\} ; \\
\lambda_{3}=\frac{1}{3}\left\{E\left[X_{3: 3}\right]-2 E\left[X_{2: 3}\right]+E\left[X_{1: 3}\right]\right\} ; \text { and } \\
\lambda_{4}=\frac{1}{4}\left\{E\left[X_{4: 4}\right]-3\left(E\left[X_{3: 4}\right]\right.\right. \\
\left.+3 E\left[Q_{2: 4}\right]-E\left[Q_{1: 4}\right]\right\} .
\end{gathered}
$$

The first L-moment is simply the mean. The second L-moment is one-half the expected difference between two randomly drawn samples, which measures dispersion of the data similar to the standard deviation. A very important property of the L-moment is that the first four L-moments can be formulated into values that are analogous - that is, they can be interpreted similarly - to the first four product moments. Summary L-moments of a sample are defined by the following:

$$
\begin{gathered}
\lambda_{1} \equiv \text { mean; } \lambda_{2} \equiv \text { L-scale; } \\
\tau_{2}=\frac{\lambda_{2}}{\lambda_{1}} \equiv \text { coefficient of L-variation (L-CV); } \\
\tau_{3}=\frac{\lambda_{3}}{\lambda_{2}} \equiv \text { L-skew; and } \tau_{4}=\frac{\lambda_{4}}{\lambda_{2}} \equiv \text { L-kurtosis. }
\end{gathered}
$$

The $\tau_{2}$ (often shown in the literature as just $\tau$ ), $\tau_{3}$, and $\tau_{4}$ statistics are sometimes known as the
"L-moment ratios," but for brevity here they are referred to as "L-moments."

The theoretical L-moments of a distribution with an expressible known inverse cumulative distribution, are calculated from:

$$
\lambda_{r+1}=\int_{0}^{1} x\left\{\sum_{k=0}^{r}(-1)^{r-k}\left(\begin{array}{l}
r \\
k
\end{array}\right)\left(\begin{array}{c}
r+k \\
k
\end{array}\right) F^{k}\right\} d F .
$$

In equation $8, x(F)$ is substituted for $x$.

To fit a distribution to data that have been summarized by L-moments, the L-moments of the data are equated to the theoretical L-moments of a probability distribution. Numerous distributions are compatible with L-moment theory such as the three-parameter generalized extreme value, generalized Pareto, Pearson Type III distributions, and four-parameter kappa distribution. The kappa distribution is a potentially attractive model for Texas annual peak-streamflow magnitude and frequency. Because the kappa distribution has four parameters instead of the usual three parameters common in distributions used in frequency analysis (for example, Asquith, 1998a; Interagency Advisory Committee on Water Data, 1982), it is a more flexible distribution and is therefore better able to model the distribution of annual peak streamflow for a station.

The flexibility of the kappa distribution is an important issue. The shapes of the distributions of regulated peak streamflow vary widely from site to site and often are quite different from those distributions suggested by experience or extreme-value theory in natural basins. The author did much exploratory research using the widely accepted technique of L-moment ratio diagrams (Vogel and Fennessey, 1993) and found that, on the diagrams, the regulated peak streamflow $\tau_{3}$ and $\tau_{4}$ pairs typically varied from the 3-parameter generalized Pareto distribution to above the 3-parameter generalized logistic distribution. The 4-parameter kappa distribution is capable of assuming the shape of either of these distributions and the solution space between them. The kappa does not have a solution above the generalized logistic distribution - this fact has important ramifications on the analysis. The additional flexibility of the kappa distribution comes at the sometimes substantial cost of having to estimate a fourth parameter, which usually cannot be done reliably with single-station data. Therefore, a regionalization technique that pools or combines annual peak-streamflow data from numerous stations should be used to estimate the L-moments. 
The kappa distribution is well documented by Hosking (1994) and Hosking and Wallis (1997, p. 202204). The kappa distribution has the following form

$$
Q(T)=\xi+\frac{\alpha}{\kappa}\left\{1-\left[\frac{1-F^{h}}{h}\right]^{\kappa}\right\},
$$

where

$Q(T)=T$-year peak streamflow associated with nonexceedance probability,

$$
F=1-1 / T \text {, and }
$$

$\xi, \alpha, \kappa, h=$ location, scale, shape 1 , and shape 2 parameters of the kappa distribution.

Hosking (1996) provides Fortran algorithms to facilitate parameter estimation using the L-moments for numerous distributions including the kappa. These algorithms could be used to make estimates of peakstreamflow frequency.

\section{Variables Representing Regulation}

Numerous characteristics or variables directly affect the degree of regulation of annual peak streamflow in a given basin. Other variables indirectly affect the degree of regulation, and others are useful surrogates for indeterminable variables. Some variables directly affecting the degree of regulation include the presence of active flood-control dams (such as TX01087 Mansfield Dam, Austin, Tex., which impounds Lake Travis), the fraction of total basin drainage area that is controlled, the flood-storage capacity of dams in the basin, and management practices if applicable. Some variables indirectly affecting the degree of regulation include the spatial distribution of passive flood control (such as small floodwaterretarding structures), the proximity of active flood control to the outlet or given stream site of the basin (amount of intervening unregulated drainage area), and the meteorological mechanisms that generate floods.

Meteorological mechanisms that generate floods include the duration, intensity, and areal extent of storms. Because of the sometimes incredibly intense and areally restricted nature of storms in Texas (Asquith, 1998a, 1999), even small unregulated areas of otherwise regulated basins can produce peak streamflow of approximately natural proportions and recurrence intervals. Use of all the identified variables is outside the scope of this investigation, even if some could be reliably quantified. Therefore, this investigation relied on variables available from the TNRCC inventory of dams to assess the effects of regulation on annual peak streamflow.

Three of the variables available from the inventory of dams are maximum capacity, normal capacity, and reservoir surface area. A fourth variable computed for this investigation is the difference between maximum capacity and normal capacity. This variable is a surrogate for the potential flood storage of a dam. Potential flood storage is a hypothetical value and therefore is only a rough estimate of the total floodcontrolling capacity in a watershed. The differences between maximum and normal capacity likely underestimate the potential flood storage for reservoirs in western parts of Texas. Western reservoirs often operate below normal capacity. Complicating matters further, regulation has universally increased since the beginning of recorded Texas history, and each watershed has a unique history and extent of dam construction. Therefore, only static time periods of dam influence are suitable for analysis.

\section{Comparison of Annual Peak Streamflow for Static Periods of Regulation}

Not all of the 192 stations in Texas that have 10 or more years of regulated record are suitable for the extended statistical analysis required to meet the objectives of this investigation. The regulated stations that are most pertinent for this investigation are those with periods of record that have two or more consecutive static or minimally changing periods of regulation. Of the 192 stations, 96 were identified as meeting this criterion. For each of the 192 original regulated stations, a time series of cumulative changes in the maximum capacity, normal capacity, and reservoir surface area was generated. An example time series for station 08136500 Concho River at Paint Rock, Tex. (see table 2) is shown in figure 3 . The changes in regulation for a particular station are not always as distinct as those shown for the Concho River at Paint Rock. For many regulated stations, less abrupt or gradual changes in regulation have occurred. Stations with gradual changes in regulation (judged subjectively) were not used.

The period of annual peak-streamflow record for the Paint Rock station, 1916-97, is indicated in the bottom graph of figure 3 . The record for the station was designated as regulated (beginning in 1931) by the 10-percent criterion because of the construction of Nasworthy Dam (TX03139), which was built in about 1930. Three static periods of regulation are identified 

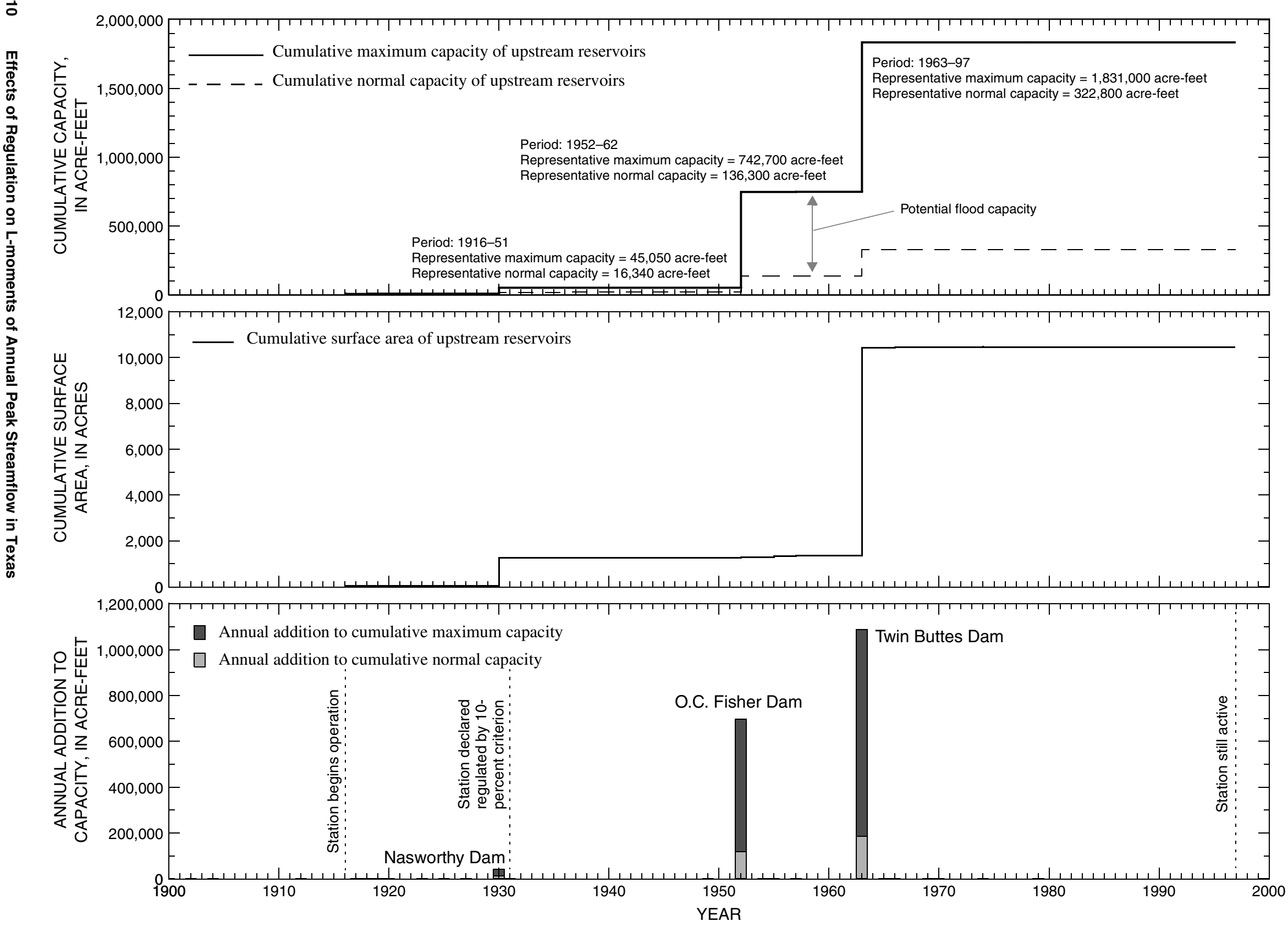

Figure 3. Example of time series of cumulative changes in regulation for station 08136500 Concho River at Paint Rock, Texas. 
(1916-51, 1952-1962, and 1963-97). It was decided, through exploratory analysis of peak-streamflow data for this station and for several others throughout the State, that the original 10-percent criterion defining regulation is probably too small to identify changes due to regulation. Preliminary analysis indicated that Nasworthy Dam did not appreciably affect annual peak streamflow for the station. Hence, the periods 1916-30 and 1931-1951 were combined. A similar combining of record was done for some of the 95 remaining regulated stations. (Such combining of record was not done for the natural peak-streamflow frequency analysis, which relied entirely on the 10-percent controlled drainage area criterion, in the section, "Estimating L-moments of Natural Annual Peak Streamflow.") It was assumed that the representative maximum and normal capacities shown in figure 3 are representative of the respective static periods. The representative maximum and normal capacities were almost always the average or near average values for the time period as dictated by the temporal distribution of dam construction and availability of peak-streamflow data.

The example cumulative regulation time series shown in figure 3 also has the station record identified. For the Paint Rock station, there are three static periods. A requirement for a station to be suitable for analysis is that the length of each static period is sufficiently long for reliable statistical analysis. This requirement improves the accuracy of comparisons of statistics of two consecutive periods. The static periods were arbitrarily designated on the basis of hydrologic judgment and comparison to nearby stations. Considerable attention was given to the selection of static periods. A representative maximum capacity and normal capacity for each period was determined. Preliminary analysis indicated that surface area of the reservoir at normal capacity provided no additional information about the effects of regulation and therefore is excluded from the remainder of this report. The 96 selected stations, the associated static periods, and maximum and normal capacities are listed in table 2.

The most succinct measure of the effects of regulation is the change or potential change in the L-moments of annual peak streamflow. If the L-moments do not change with a change in regulation characteristics, then the logical conclusion is that the change in regulation does not affect annual peak streamflow. (It is possible that a given degree of regulation affects only parts of the hydrologic regime, such as low flows, even if the larger [greater than median or 2-year] annual peak streamflows are apparently unaffected.) However, if the annual peak-streamflow statistics do change, then the logical conclusion is that the given degree of regulation does affect the peak streamflow.

To quantify the effects of regulation, the changes in L-moments of annual peak streamflow for each available pair of consecutive static periods were determined. The changes in the L-moments of the streamflow, such as the changes in mean peak streamflow, were related to changes in reservoir characteristics (hence, degree of regulation). For each of the regulated stations with two or more static periods of regulation, the L-moments of the streamflow for each static period were estimated. Subsequently, the change in the L-moments between two consecutive static periods as well as the change in reservoir characteristics were calculated. Also, a statistical test, the Mann-Kendall test (see the following section of this report) was done along the annual peakstreamflow time series of each pair of consecutive static periods.

\section{Analysis of Trends}

The Mann-Kendall test, or Kendall correlation (Helsel and Hirsch, 1992; Hollander and Wolfe, 1973), was used to indicate whether the annual peak streamflows for a station have historical trends with time. The Mann-Kendall test is a nonparametric, rank-based hypothesis test. That is, no assumption of normally distributed data is required, and the test is based on the ranks of the data rather than on the actual data. The data are tested to determine whether the null hypothesisthat there is no trend in the data-is supported by the strength of the evidence provided by the data. The outcome of the test is a decision whether or not to reject the null hypothesis in favor of an alternative hypothesis. The alternative hypothesis is that there is a downward trend in the data. The Mann-Kendall test commonly is used in a two-tailed fashion-that is, either upward or downward trends are of interest. However, for the case of streamflow that is potentially affected by regulation, a logical expectation is that potential trends in annual peak streamflow are downward. Therefore, a one-tailed test was used in the analysis of trends in annual peak streamflow. The decision to reject the null hypothesis in favor of the alternate hypothesis is made on the basis of the $\mathrm{p}$-value from the test. The $\mathrm{p}$-value indicates the strength of the evidence against the null hypothesis; the smaller the p-value, the stronger the evidence against the null hypothesis. Downward trends were identified as 
significant for this report if a computed p-value was less than or equal to 0.10 . The Mann-Kendall test was chosen over rank-sum nonparametric tests because the Mann-Kendall test is sensitive to both discrete and gradual changes in a random variable (peak streamflow) in time. Regulation is a temporal changing feature of many basins considered here; hence the Mann-Kendall test is preferred.

\section{EFFECTS OF REGULATION ON L-MOMENTS OF ANNUAL PEAK STREAMFLOW}

\section{Estimating L-moments of Natural Annual Peak Streamflow}

A multiple weighted least-squares regression was done for each of the designated northeast, southeast, and west regions. Multiple regression analysis establishes a mathematical relation between one dependent and two or more independent variables. The mean, L-CV, L-skew, and L-kurtosis were used as dependent variables; and the contributing drainage area, basin shape factor, and stream slope basin characteristics were used as independent variables. Logarithmic transformations of the mean and the independent variables were used to increase the linearity of the relation between the dependent and independent variables.

A step-forward weighted least-squares (WLS) regression procedure (Statware, Inc., 1990) was used to develop equations to estimate the L-moments for stream sites in natural basins in Texas. The 367 natural streamflow-gaging stations previously identified (table 1) were used. In WLS regression, each datapoint is given a weight that is representative of the relative accuracy of the dependent variable. Greater weights are assigned to values that have greater accuracy. Because the sampling variance (error) of the L-moments decreases with increasing sample size, the number of natural annual peak-streamflow years for each station was used as the weighting variable. The equations to estimate the L-moments of natural annual peak streamflow for each region are listed in table 4 (at end of report). Diagnostic statistics, R-squared, and standard error, are reported in table 4 . The residuals for each equation were analyzed (results not presented in this report), and the results indicate that each equation has no substantial bias for the range of independent variables included.

For the equations estimating the mean annual peak streamflow, each basin characteristic is included.
The p-values for each coefficient are less than 0.10, except for the basin shape factor for the northeast region ( $\mathrm{p}$-value $=0.37)$. Shape factor was retained in the northeast equation for consistency with mean equations for the other two regions.

For the northeast region $\mathrm{L}-\mathrm{CV}$ equation, the coefficient on drainage area had a p-value of 0.04 , and the other coefficient $\mathrm{p}$-values were greater than 0.70 . For the southeast region L-CV equation, all three basin characteristics are included. The p-value for each coefficient was less than 0.06 . For the west region $\mathrm{L}-\mathrm{CV}$ equation, contributing drainage area and basin shape factor are included. The $\mathrm{p}$-value for each coefficient is less than 0.11 , not 0.10 as described for the mean or L-skew equations.

No independent variables were significant for estimating L-skew for the northeast region. Therefore, the regional mean value was used. The regional standard deviation for L-skew provides a means for assessing relative error. For the southeast region L-skew equation, contributing drainage area and stream slope are included. The p-value for each coefficient is less than 0.10 . Only basin shape factor, with a p-value of 0.01 , is included for the west region L-skew equation.

No independent variables were significant for estimating L-kurtosis for any of the three regions. Therefore, the regional mean value was used. The regional standard deviation for L-kurtosis provides a means for assessing relative error.

The largest errors were for the west region equations. This is likely because the west region is the largest region and, therefore, has the greatest heterogeneity among watersheds. Also, semiarid areas such as the west region show more year-to-year variation in annual peak streamflow than do more humid areas, which is evident because the west region $\mathrm{L}-\mathrm{CV}(0.551)$ is greater than the L-CV (0.434 and 0.462) for the other two regions.

The equations can be used to estimate the L-moments of the annual peak streamflow for gaged and ungaged sites in their respective regions. From the estimates of the L-moments, estimates of the parameters for a distribution can be derived. To illustrate application, suppose the kappa distribution is suitable for northeast region station 08018730 Burke Creek near Yantis, Tex., with a contributing drainage area, basin shape factor, and stream slope of $33.1 \mathrm{mi}^{2}, 2.88$, and 10.41 feet per mile (ft/mi), respectively. The natural L-moments are 2,670 cubic feet per second $\left(\mathrm{ft}^{3} / \mathrm{s}\right)$; 0.449 ; 0.315 ; and 0.203 for the mean, L-CV, L-skew, 
and L-kurtosis, respectively. The corresponding parameters of the kappa distribution are $1,226 \mathrm{ft}^{3} / \mathrm{s} ; 1,609$ $\mathrm{ft}^{3} / \mathrm{s} ;-0.148$; and 0.327 for $\xi, \alpha, \kappa$, and $h$, respectively. The 2-year peak thus is $2,020 \mathrm{ft}^{3} / \mathrm{s}$, and the 100 -year peak is $11,800 \mathrm{ft}^{3} / \mathrm{s}$. For comparison, the 2-year and 100 -year peaks from the regional regression equations of Asquith and Slade (1997) are 1,680 and 11,350 ft $\mathrm{ft}^{3} / \mathrm{s}$, respectively. The equations and L-moment compatible distributions such as the kappa distribution provide an alternative to other methods, principally Asquith and Slade $(1997,1999)$ and a substantially independent method from the Asquith and Slade reports to estimate natural peak-streamflow frequency for stream sites in Texas. It is unknown to what extent the natural L-moment based peak-streamflow frequency estimates might differ from those derived using the methods of Asquith and Slade (1997) or (1999) or derived specifically from gaged data. Substantial variation among estimates from the methods is expected.

\section{Quantifying Regulation Effects on L-moments of Natural Annual Peak Streamflow}

Quantifying the effects of regulation on annual peak streamflow is a difficult problem. The degree of regulation can be extremely site specific for many streams and provide "limited" to "no" statistical transferability. For other regulated sites, however, streamflow statistics and statistics reflecting the effects of regulation on peak streamflow are transferable. For a stream site that has passive flood control, the effect of regulation would be to systematically reduce the mean annual peak streamflow, to potentially reduce the variability (L-scale, therefore L-CV) of the annual peak streamflows, and to possibly alter the higher moment ratios (L-skew and L-kurtosis). The amount of systematic reduction or other changes in the L-moments might correlate to changes in regulation (reservoir characteristics). If suitable correlations exist, then the degree of regulation is statistically transferable to sites that have similar regulation.

However, for a stream site that has active flood control, regulation would affect all the streamflow statistics in unpredictable and potentially temporally changing ways; although for Texas, it might be expected that the mean and the variability of the annual peak streamflow would be reduced because of competing water-supply and flood-control regulatory actions. Water-supply actions, including those associated with irrigation, tend to increase the smaller annual peaks, and flood-control actions tend to decrease the larger annual peaks. Thus the variability is reduced by this two-tailed compression of the peak-streamflow distribution. Transferability of the relation between annual peakstreamflow statistics and active flood control is, therefore, more limited.

Because of highly site-specific characteristics of regulation, any attempt to generalize the relation between degree of regulation and annual peak streamflow should be approached with caution. Though the analysis presented in this section provides (to the author's knowledge) the first statewide regional assessment of regulation on annual peak streamflow, the assessment is made difficult by factors such as proximity of the stream site to active flood-control dams, degree of direct influence of mankind on the hydrologic regime, and unpredictable characteristics of the basin.

For each of the static or near-static periods of regulation (discussed in a previous section in this report), the change in maximum capacity, change in normal capacity, and change in the difference between maximum and normal capacity (potential flood storage) are graphed as a function of drainage area upstream of each site (fig. 4a, b, c). Because of the large range in drainage areas, the reservoir-characteristic changes were divided by contributing drainage area to standardize the regulation variables for contributing drainage area. The units of the reservoir-characteristic changes thus are acre-feet per square mile. The three graphs in figure 4 also indicate the stations that have MannKendall p-values less than 0.10 (trend) and those that do not (no trend). An apparent downward trend of each ordinate (vertical axis) is a product of plotting a function of Area $^{-1}$ against Area. The Mann-Kendall test indicates (fig. 4c), as expected, that changes in the difference between maximum and normal capacity generally are greater for those stations (with the same drainage area) that have a trend than for those that do not have a trend.

Similar plots for each region are shown in figures 5-7. For each of the regions, the change in maximum capacity and change in difference between maximum and normal capacity shows the most contrast between trend and no-trend stations. Some regional differences are that the drainage areas for the southeast region stations generally are larger. Most of the stations in the semiarid west region have trends, whereas most stations in the other two regions do not. Since runoff rates are much smaller in the west, a reservoir of a given size controls a larger portion of the runoff in the west. Stated another way, the effectiveness of a reservoir is a 

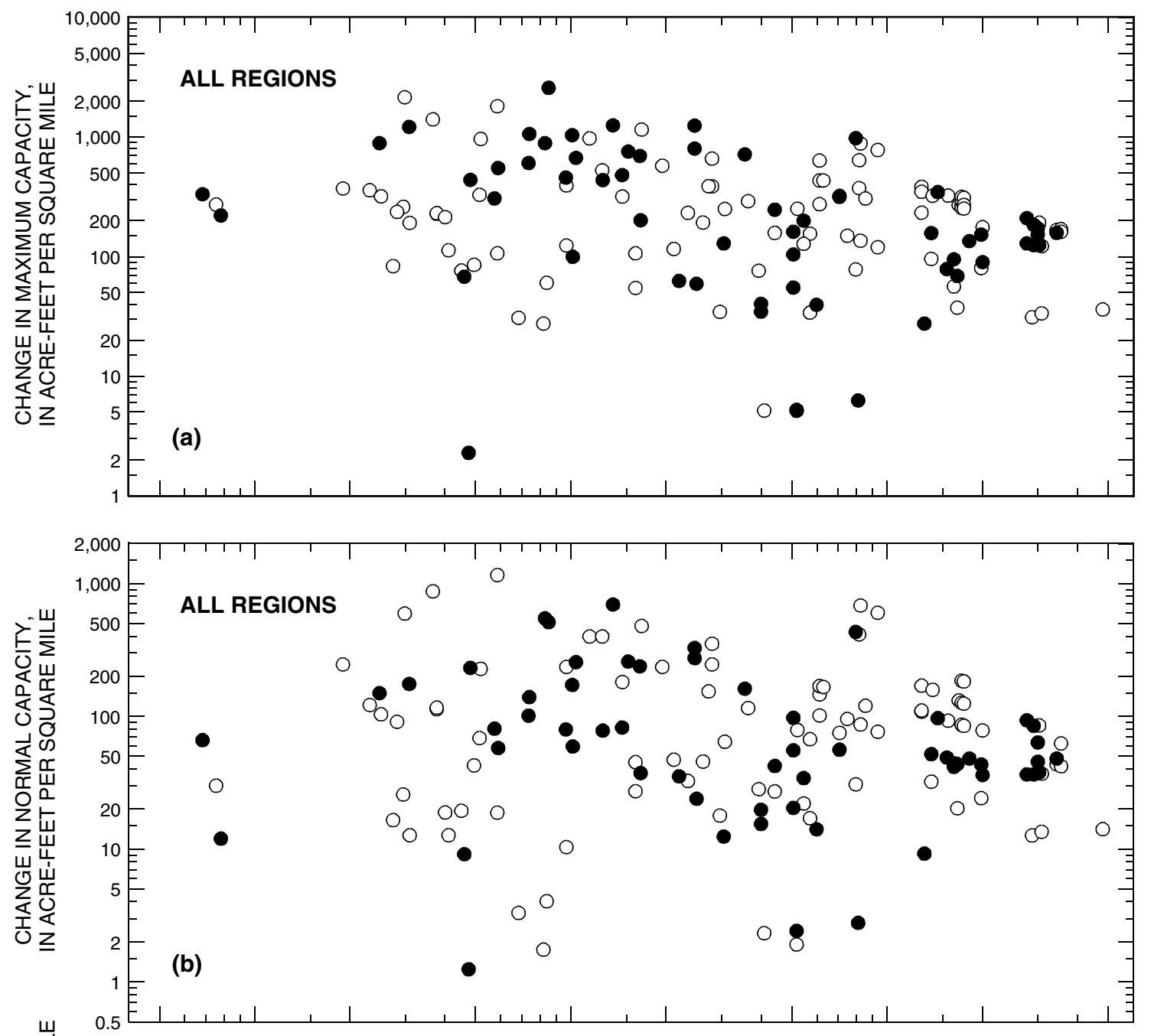

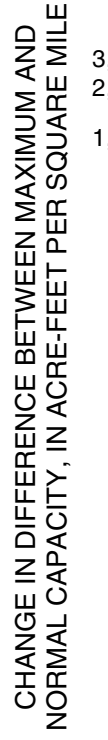

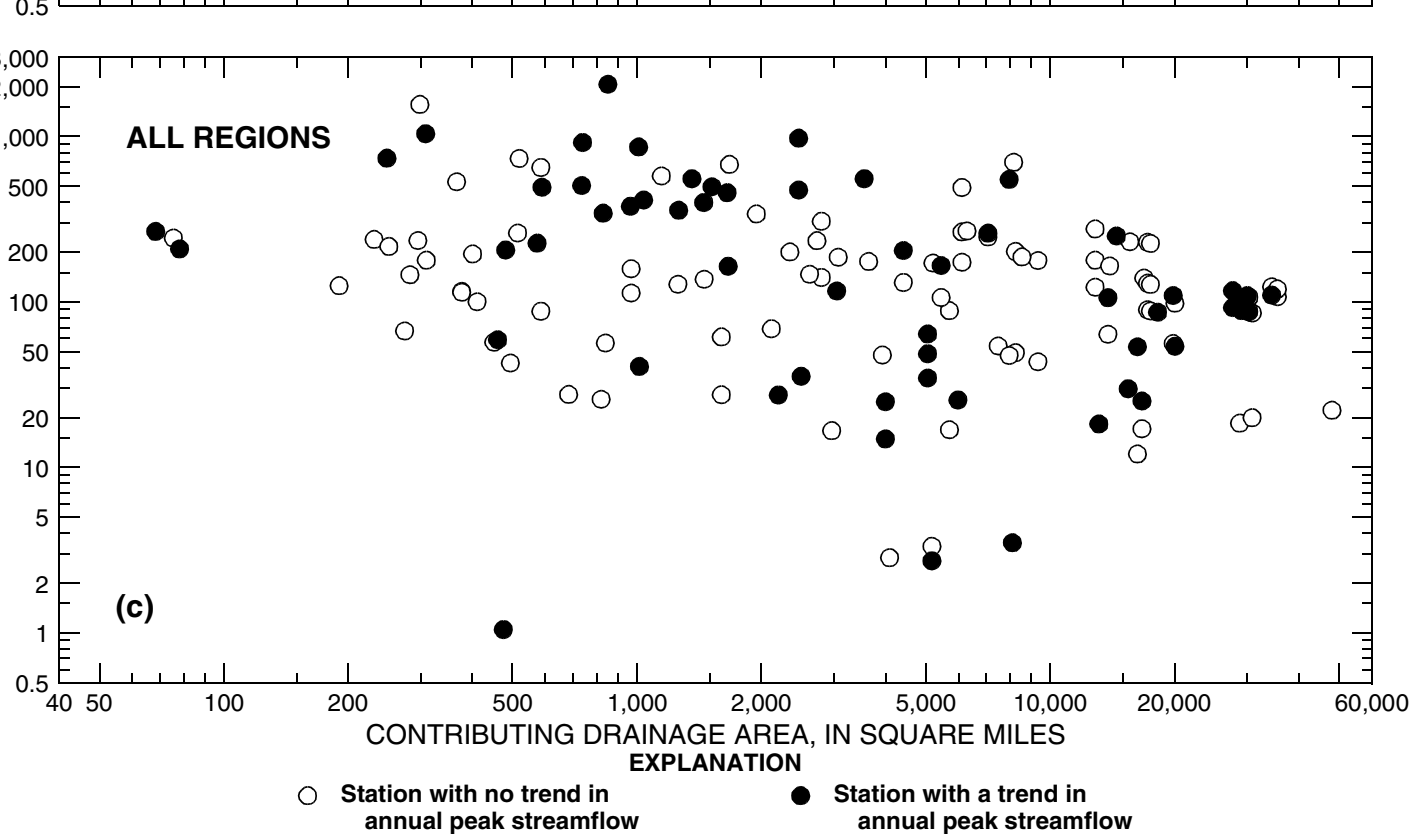

Figure 4. Relation between (a) change in maximum capacity, (b) change in normal capacity, and (c) change in difference between maximum and normal capacity to contributing drainage area in all regions. 

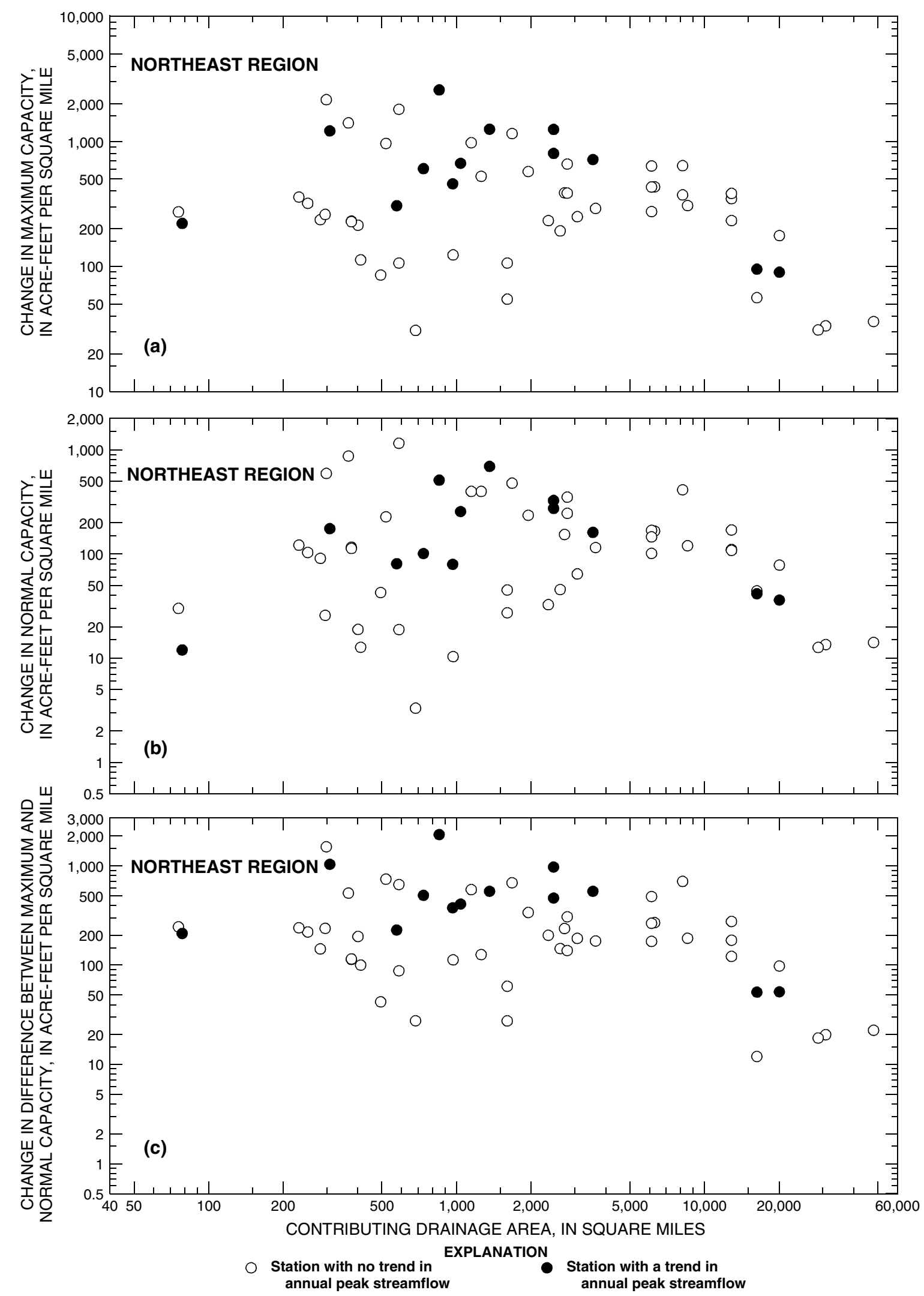

Figure 5. Relation between (a) change in maximum capacity, (b) change in normal capacity, and (c) change in difference between maximum and normal capacity to contributing drainage area in northeast region. 

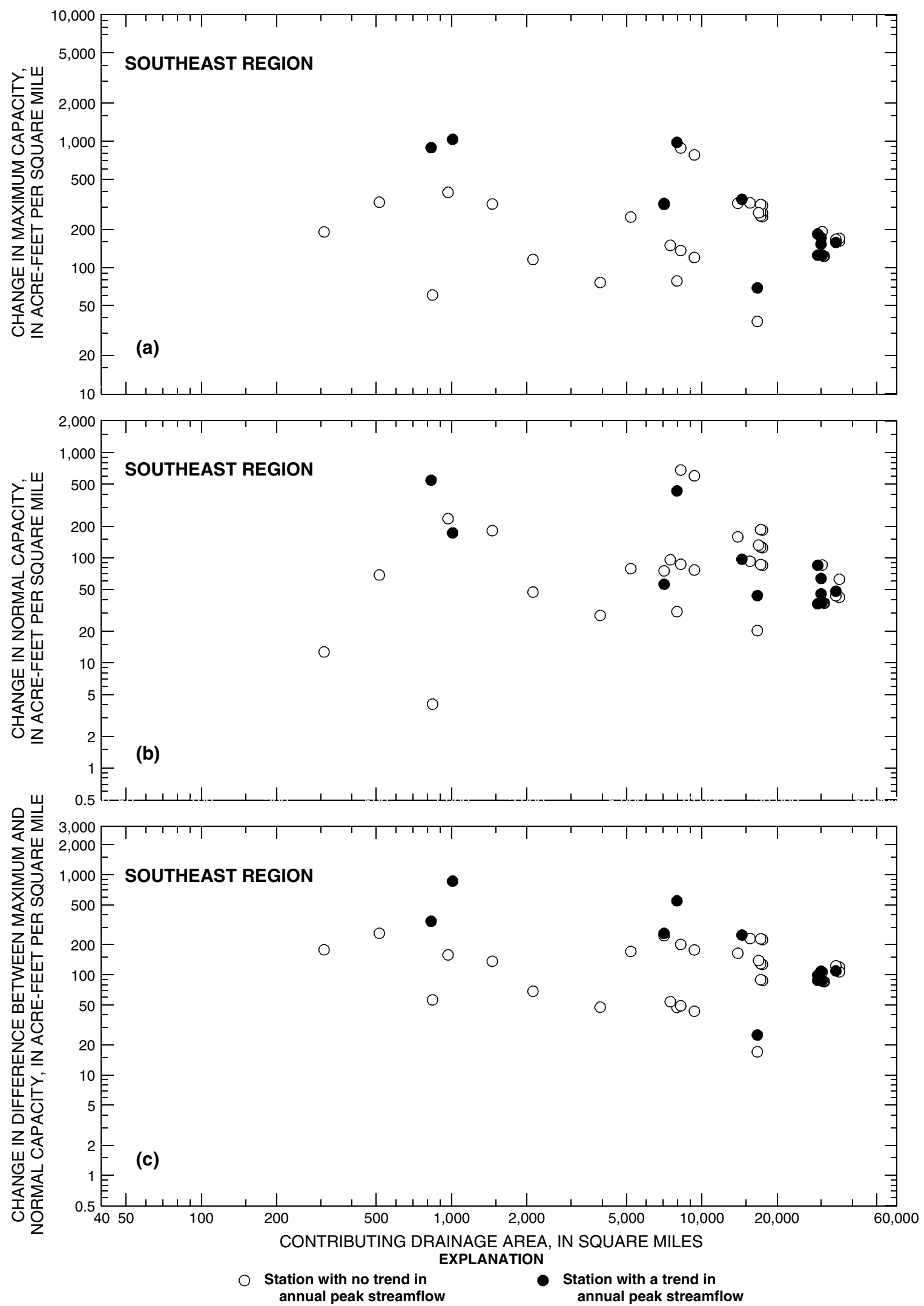

Figure 6. Relation between (a) change in maximum capacity, (b) change in normal capacity, and (c) change in difference between maximum and normal capacity to contributing drainage area in southeast region. 

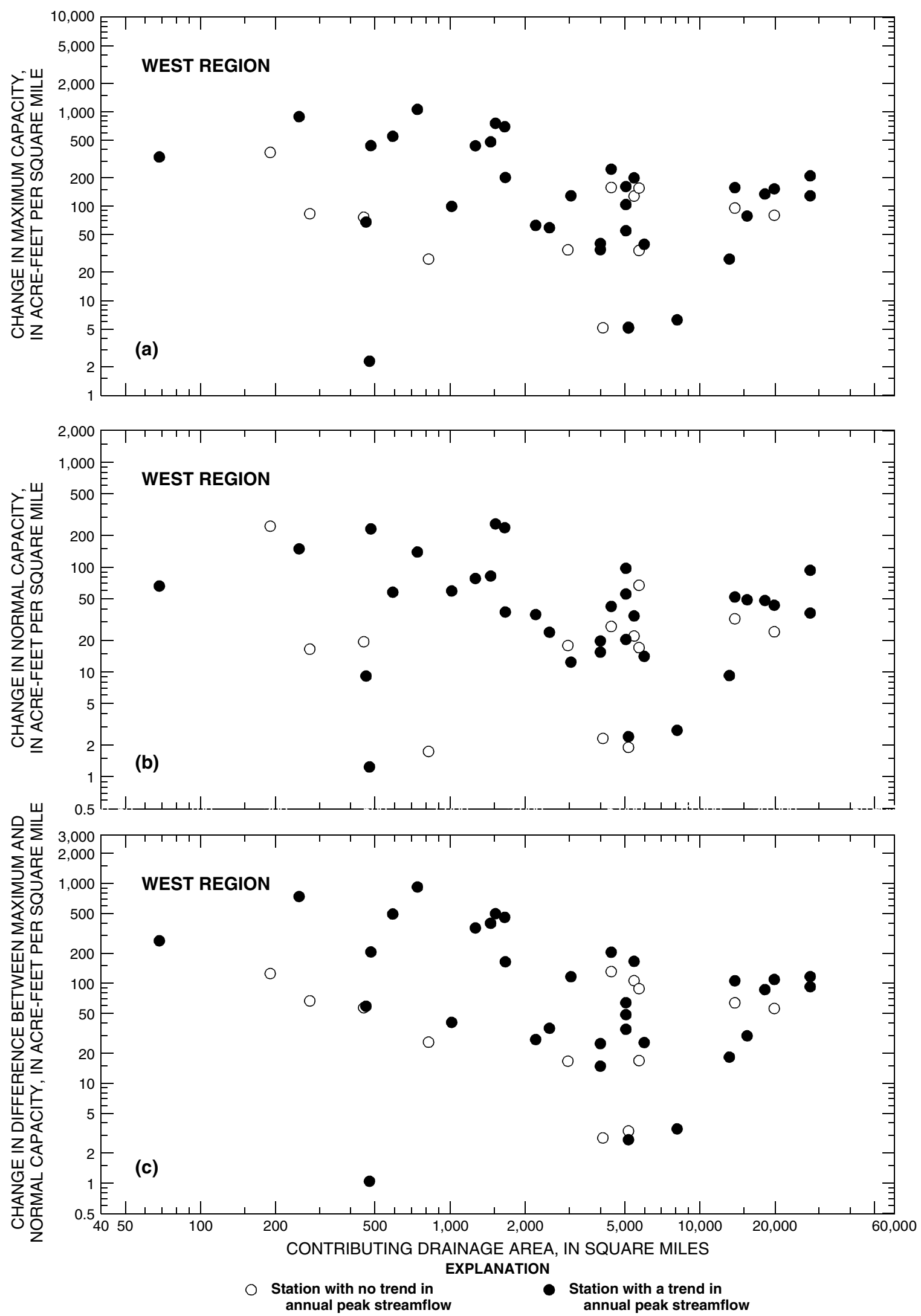

Figure 7. Relation between (a) change in maximum capacity, (b) change in normal capacity, and (c) change in difference between maximum and normal capacity to contributing drainage area in west region. 
function of its capacity to expected runoff volumes, and runoff volumes are low (relative to the east) in west Texas. In conclusion, reservoirs in the west region affect peak streamflow to a greater degree than those in the northeast or southeast regions.

\section{Adjusting L-moments of Natural Annual Peak Streamflow for Potential Regulation Effects}

To adjust the L-moments and L-moment ratios of natural annual peak streamflow for potential effects of regulation (whether estimated from the equations [table 4] or through other methods), considerable exploratory analysis of the change in L-moments and their relation to change in regulation was required. This section documents the most important aspects of the analysis and presents graphs showing the relation between change in L-moments and change in maximum capacity, change in normal capacity, change in difference between maximum and normal capacity, and contributing drainage area (figs. 8-13).

The relations between change in the mean annual peak streamflow to change in maximum capacity and to change in normal capacity are shown in figure 8 . The relation between change in mean annual peak streamflow and change in the difference between maximum and normal capacity is shown in figure 11a. In each figure, the change in mean peak is graphed as a function of one of the three types of reservoir-characteristic changes. Also in each figure, the region for each point is indicated and whether a trend in annual peak streamflow exists for two consecutive static reservoir periods.

Clearly, a decreasing relation between change in the mean and degree of regulation (as evidenced by trends in the three reservoir-characteristic changes) exists. Few points plot above the zero-change line. Only one of the points that plot above the zero-change line was from data having a trend. The points that have trends generally show greater decreases in the mean peak with regulation than points that do not have trends. The change in normal capacity (fig. 8b) does not indicate as strong a mean-peak-to-regulation relation as does the maximum capacity (fig. 8a). The change in normal capacity also does not show as strong a relation as does the change in difference between maximum and normal capacity (fig. 11a). Accordingly, the normal capacity is not considered further.

The change in difference between maximum and normal capacity (fig. 11a) was selected rather than the change in maximum capacity (fig. 8a) to estimate the change in mean annual peak streamflow for ungaged sites for a specific region and for change in regulation. The relation between mean annual peak streamflow and change in regulation is better defined in figure $11 \mathrm{a}$. It would be expected, on a conceptual basis, that potential flood storage in a basin (difference between maximum and normal capacity per square mile) would have a more direct influence on annual peak streamflow than maximum or normal capacity alone does.

Two graphically fitted line segments in figure 11a reflect the approximate relation between change in mean annual peak streamflow and potential flood storage. The equations for the lines are shown on the figure. For potential flood storage values less than about 20 acre- $\mathrm{ft} / \mathrm{mi}^{2}$, the change in the mean is zero. For potential flood storage greater than about 400 acre$\mathrm{ft} / \mathrm{mi}^{2}$, the results of this report are considered not applicable.

Figure 9 shows the relation between change in L-CV of annual peak streamflow to change in maximum capacity (fig. 9a) and change in normal capacity (fig. 9b). The relation between change in $\mathrm{L}-\mathrm{CV}$ and change in the difference between maximum and normal capacity is shown in figure $11 \mathrm{~b}$. The region for each point is indicated and whether a trend was detected in annual peak streamflow for two consecutive static reservoir periods. From the figures $(9 a, b ; 11 b)$, it is evident that little-to-no functional relation exists between the change in $\mathrm{L}-\mathrm{CV}$ and the change in regulation. Thus, the magnitude of L-CV probably is not influenced by regulation. This conclusion is consistent for each region, whether or not a trend was detected. However, a closer inspection of the figures indicates that an argument could be made that a very subtle decreasing relation between change in $\mathrm{L}-\mathrm{CV}$ and change in regulation might exist. This is only partially consistent with a logical assessment of the effects of regulation on L-moments of annual peak streamflow and requires some additional discussion.

A decrease in $\mathrm{L}-\mathrm{CV}$ with regulation might be expected because a decrease in $\mathrm{L}-\mathrm{CV}$ implies a decrease in relative variability of the annual peak streamflow, which reflects the peak-streamflow buffering capability of reservoirs when they are used for competing flood-control and water-supply objectives. What is not consistent with a decreasing relation between $\mathrm{L}-\mathrm{CV}$ and regulation is that the relation would not be expected to exist above the zero-change line (positive changes in $\mathrm{L}-\mathrm{CV}$ ). Factors that cause a positive change in $\mathrm{L}-\mathrm{CV}$ include either a decrease in the 

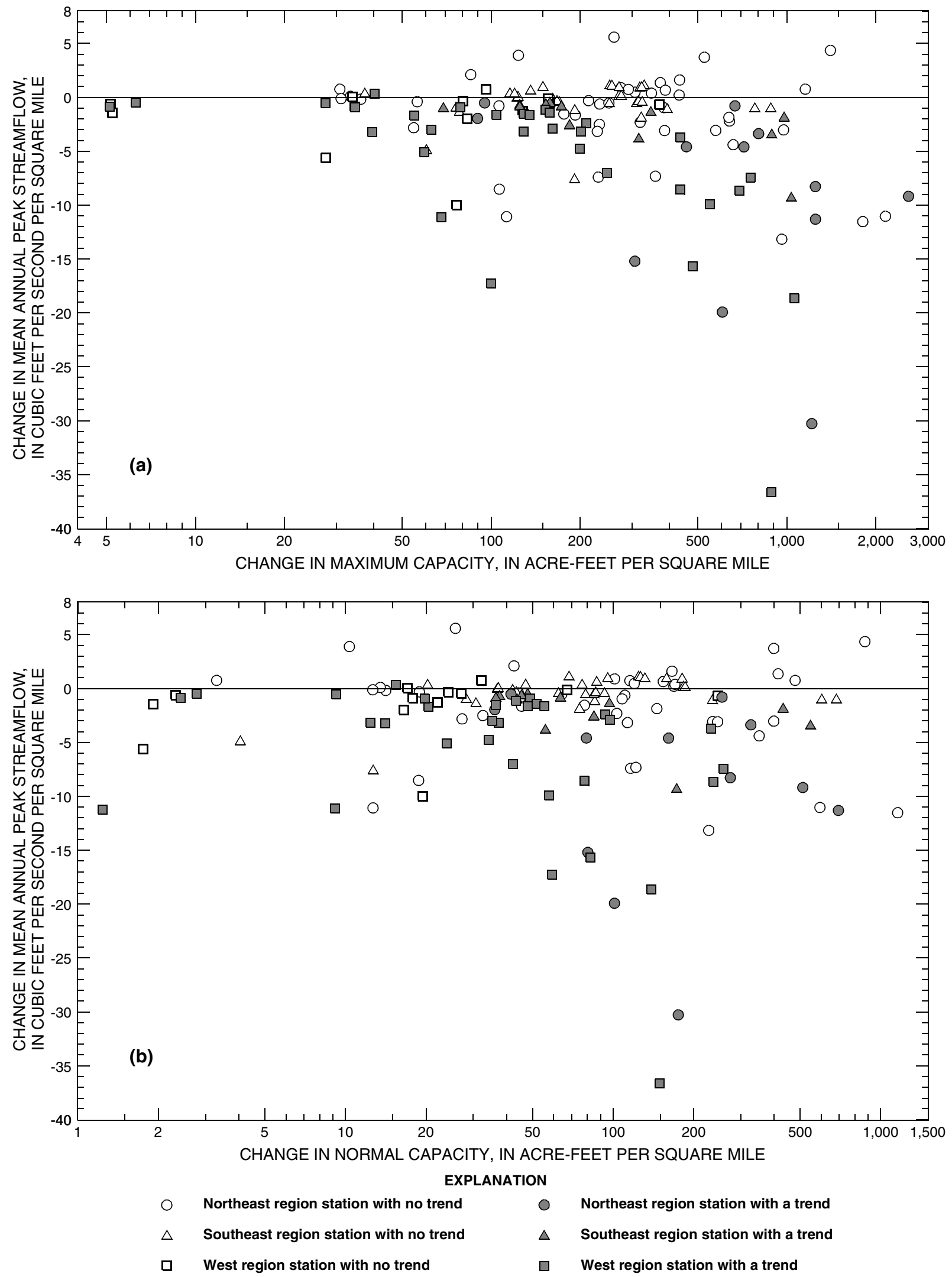

Figure 8. Relation between change in mean annual peak streamflow to (a) change in maximum capacity and (b) change in normal capacity 

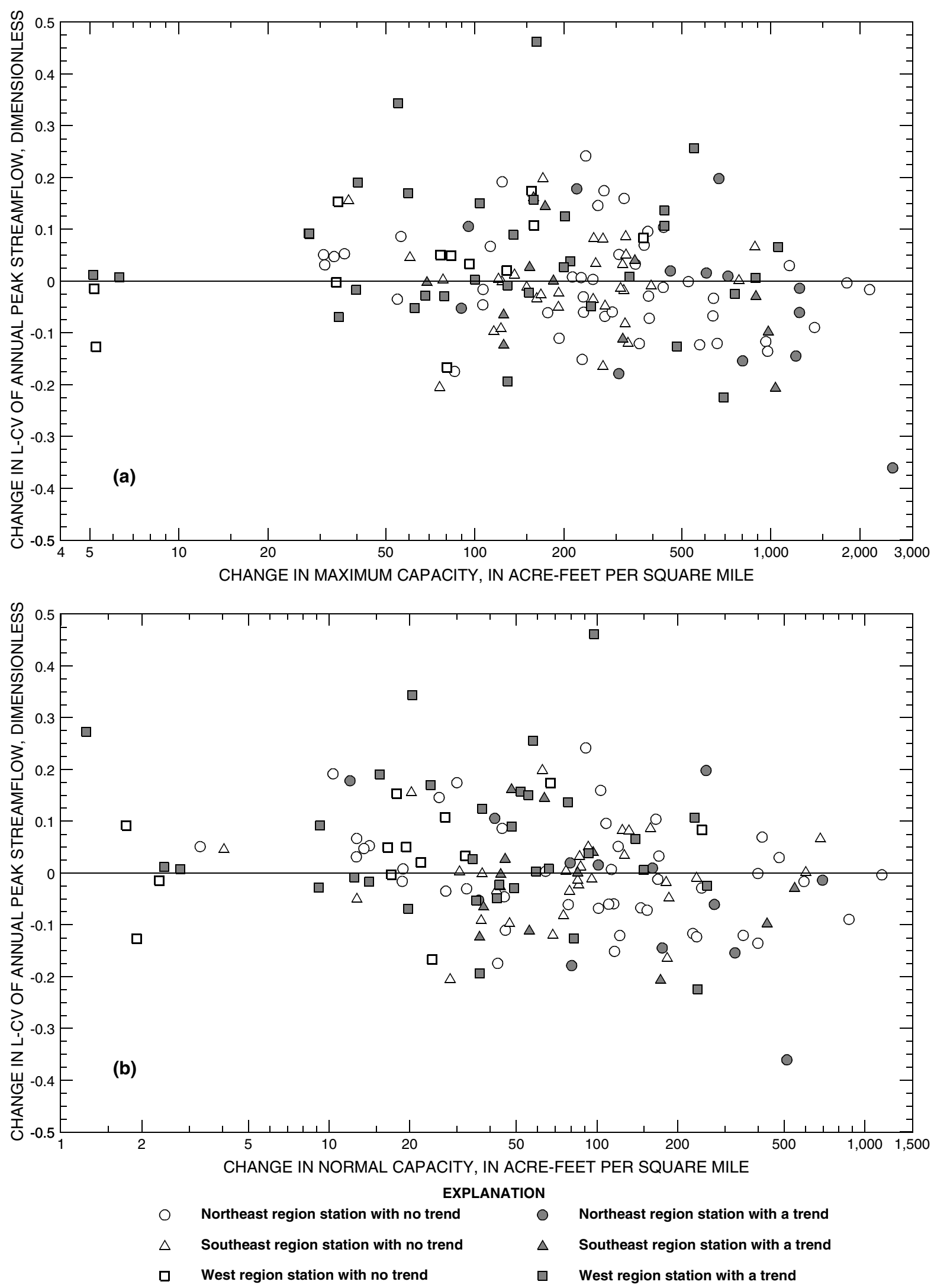

Figure 9. Relation between change in L-CV of annual peak streamflow to (a) change in maximum capacity and (b) change in normal capacity. 

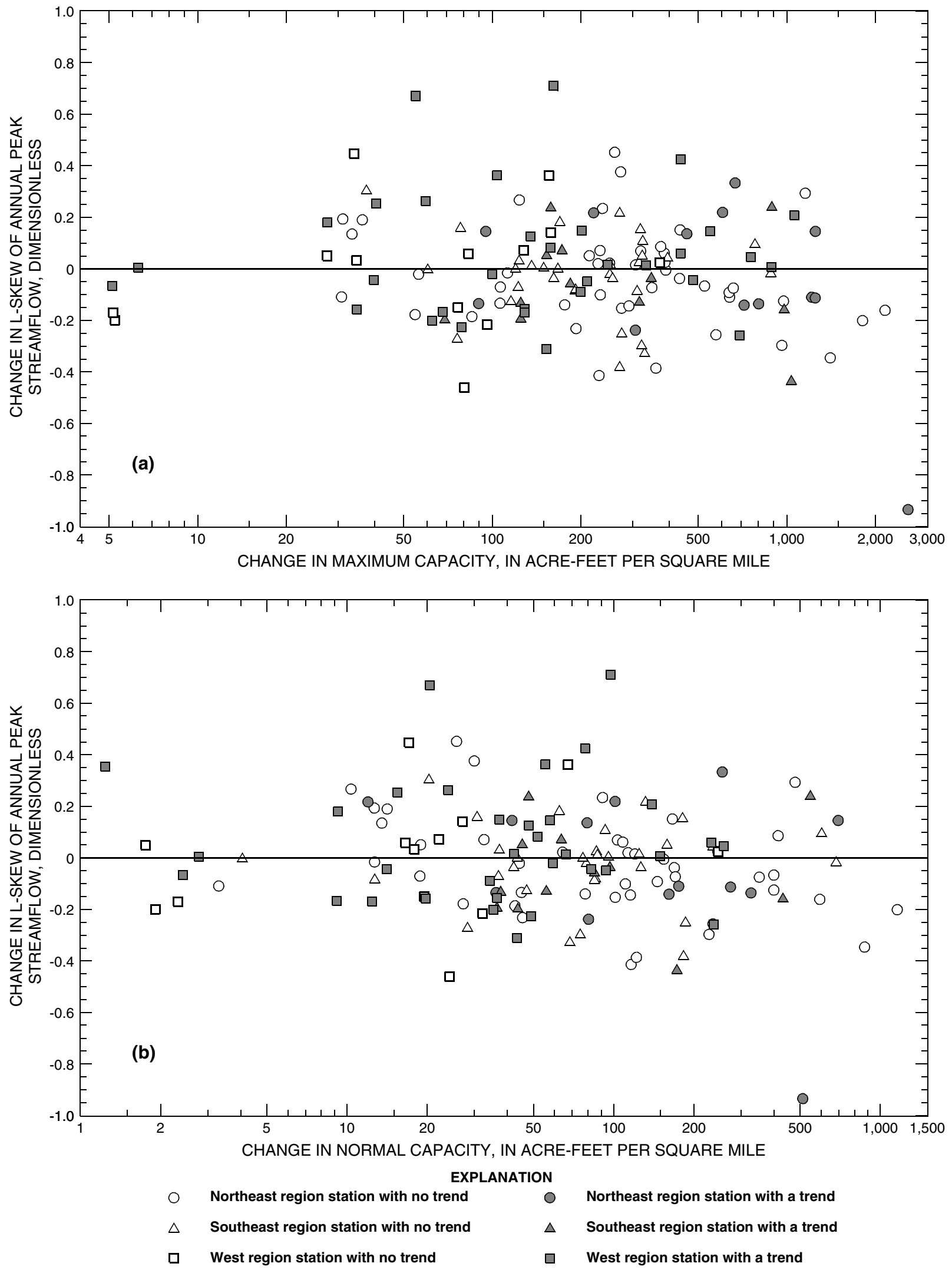

Figure 10. Relation between change in L-skew of annual peak streamflow to (a) change in maximum capacity and (b) change in normal capacity. 

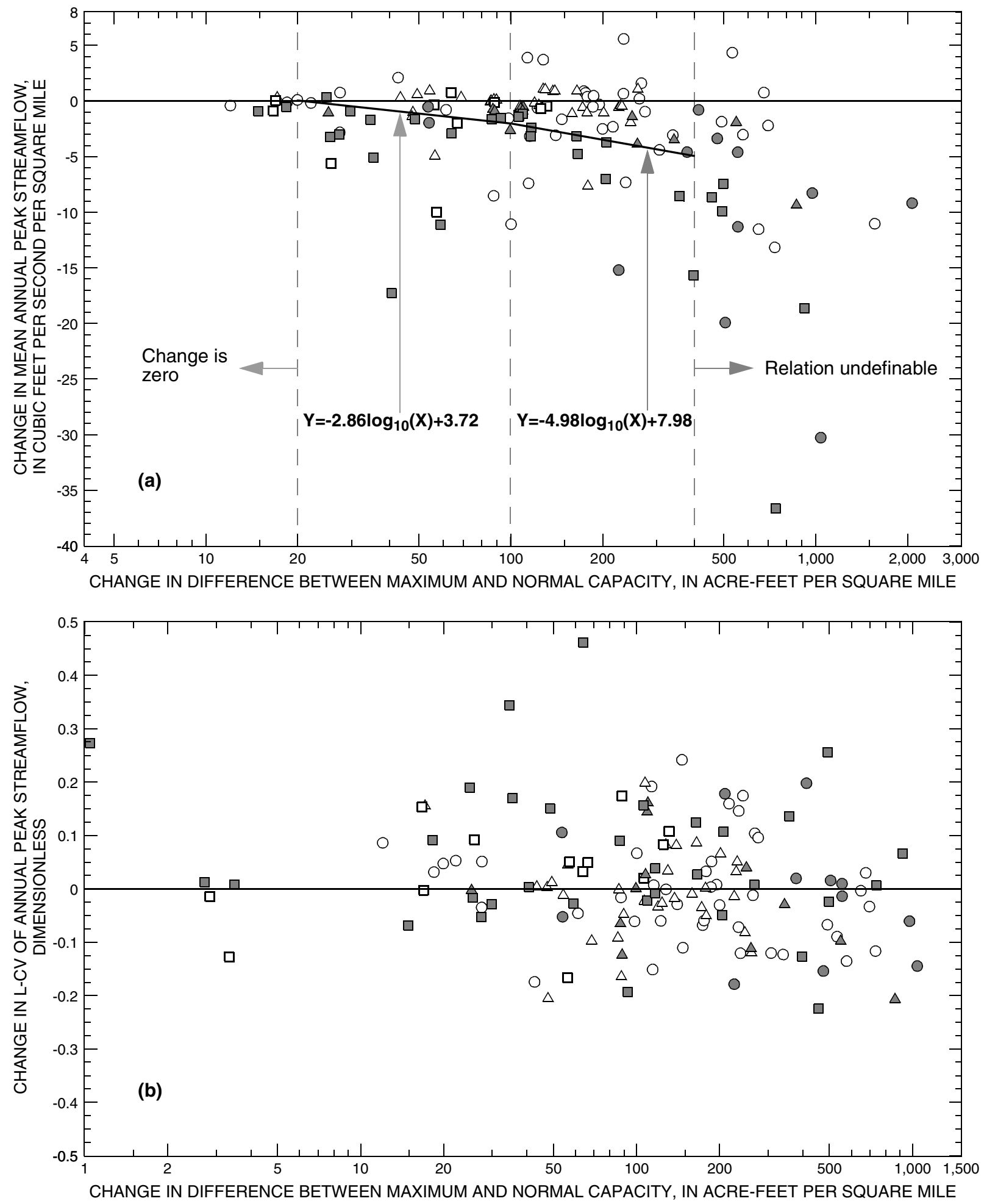

$\begin{array}{llll} & \text { EXPLANATION } & \\ & \text { Northeast region station with no trend } & O & \text { Northeast region station with a trend } \\ \triangle & \text { Southeast region station with no trend } & \Delta & \text { Southeast region station with a trend } \\ \square & \text { West region station with no trend } & \square & \text { West region station with a trend }\end{array}$

Figure 11. Relation between (a) change in mean annual peak streamflow and (b) change in L-CV of annual peak streamflow to change in difference between maximum and normal capacity. 


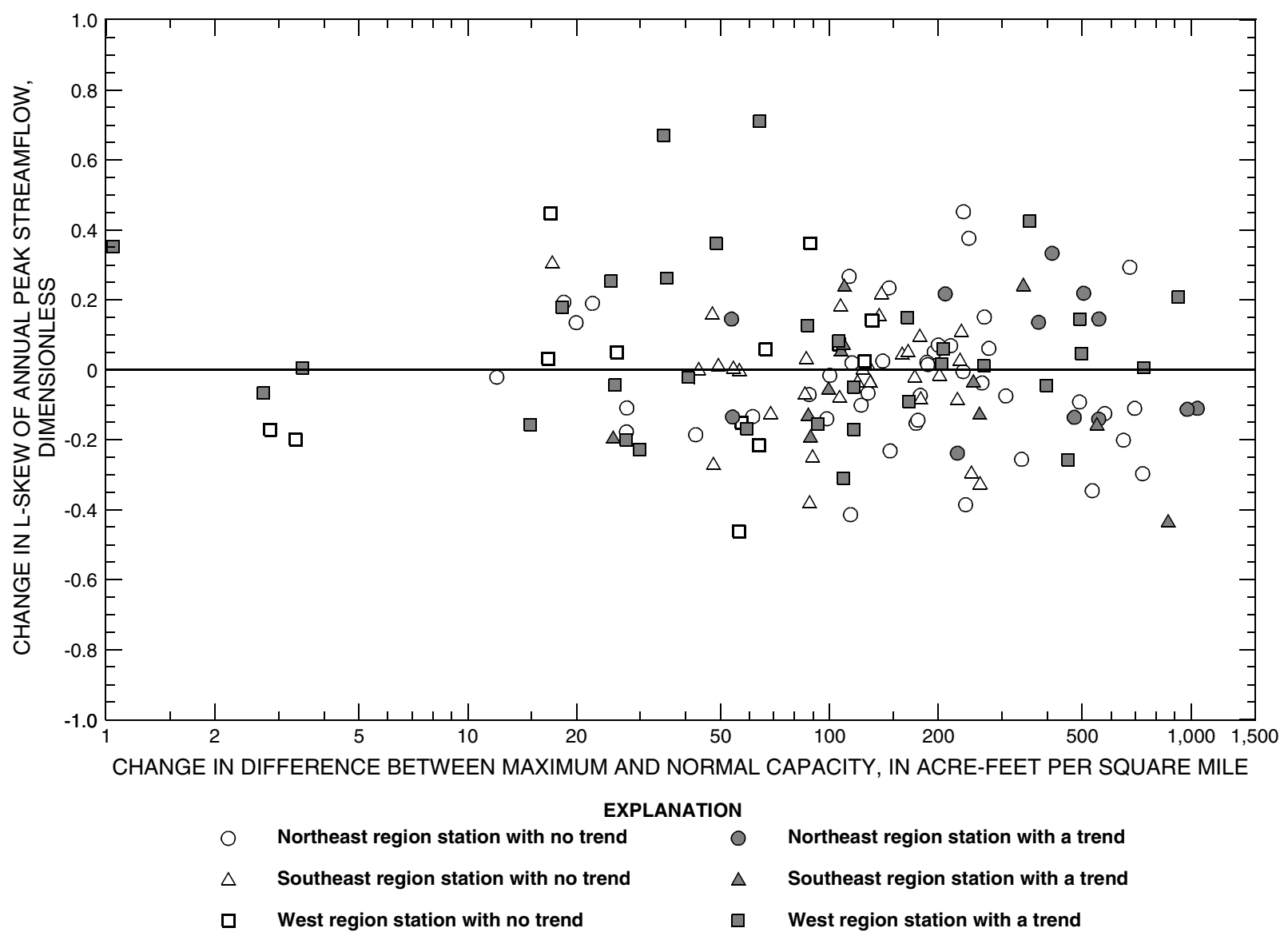

Figure 12. Relation between change in L-skew of annual peak streamflow and change in difference between maximum and normal capacity.

mean, an increase in the L-scale (variation), or both. Regulation is often thought to decrease the mean, which could increase L-CV if the natural or "background" variation remains constant. Natural variation is an inherent function of precipitation variability. Whether a trend was detected provides little additional insight. The author concluded, for purposes of application, that there is negligible or no relation of $\mathrm{L}-\mathrm{CV}$ to regulation.

The relation between change in L-skew of annual peak streamflow to change in maximum capacity and change in normal capacity is shown in figure 10, and the relation between change in the L-skew of annual peak streamflow and change in the difference between maximum and normal capacity is shown in figure 12. In each figure, the change in L-skew is graphed as a function of one of the three types of reservoir-characteristic changes. Also, the region for each point is indicated and whether a trend was detected in annual peak streamflow for two consecutive static reservoir periods. From the figures, it is evident that little or no functional relation exists between the change in L-skew, hence magnitude of L-skew, and changes in regulation. This holds true for each region, whether or not a trend was detected. On the basis of the data available, the conclusion is that the degree of regulation in a particular basin has negligible influence on the L-skew for the basin. Similar results were seen for L-kurtosis, and the same conclusion was made (results not presented here).

Additional comparisons between change in the mean and L-CV of annual peak streamflow and contributing drainage area are needed to assess the effects of basin scale on these statistics. Figure 13 provides the comparison. As with figures 8-12, the region for each point is indicated and whether a trend was detected in annual peak streamflow for two consecutive static reservoir periods. From figure 13a, it is apparent that a strong nonlinear decreasing-magnitude-withincreasing-area relation exists in points having trends for drainage areas as large as about $8,000 \mathrm{mi}^{2}$. Specifically, the largest changes in the mean occur for the 

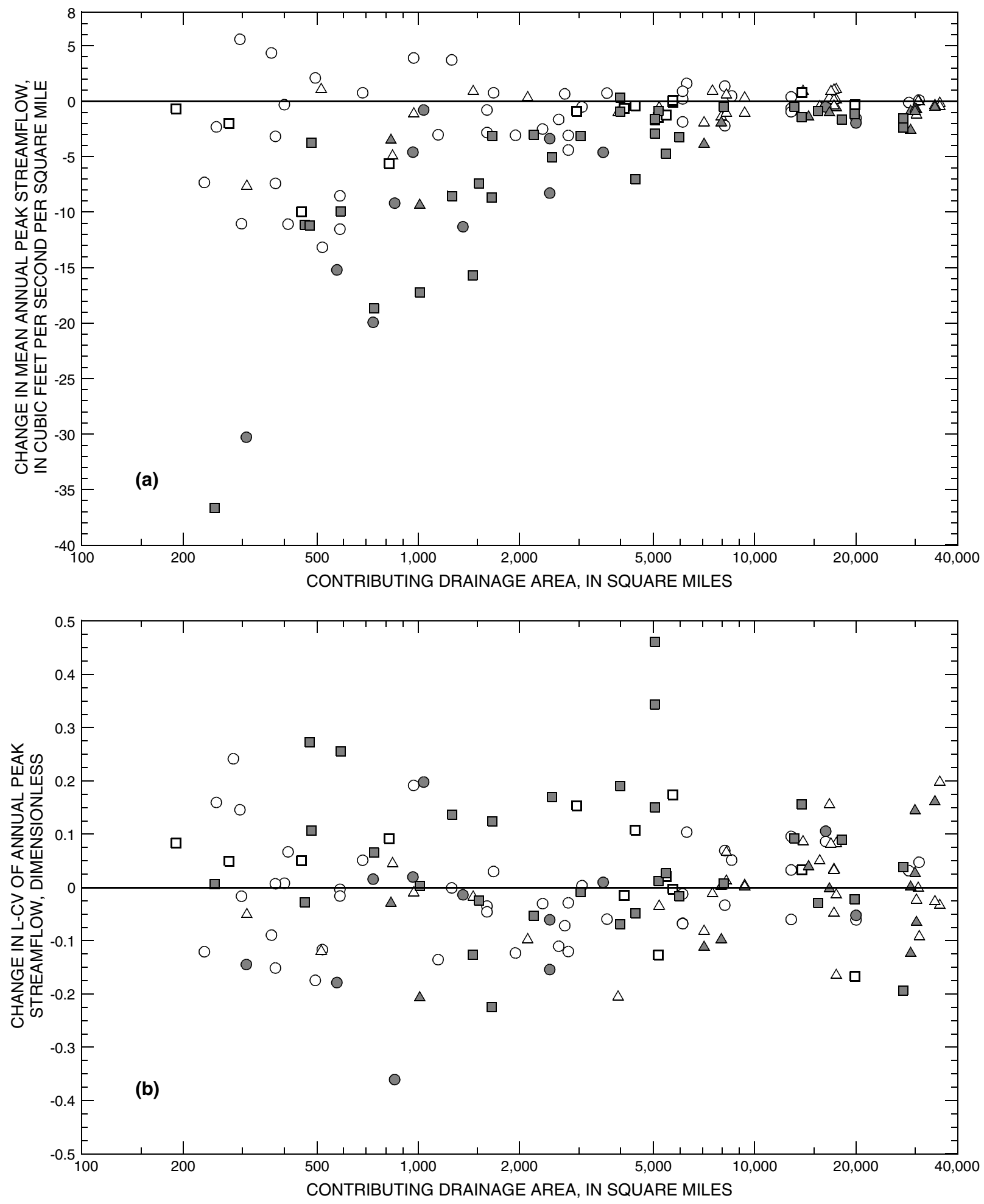

$\begin{array}{llll} & \text { Northeast region station with no trend } & & \text { Northeast region station with a trend } \\ \triangle & \text { Southeast region station with no trend } & \Delta & \text { Southeast region station with a trend } \\ \square & \text { West region station with no trend } & \square & \text { West region station with a trend }\end{array}$

Figure 13. Relation between (a) change in mean annual peak streamflow and (b) change in L-CV of annual peak streamflow to contributing drainage area. 
smallest basins. It is not apparent from figure 13a whether a similar relation exists for points without a trend. This observation is important because the drainage area is on the horizontal axis, and the inverse of area is on the vertical axis. Figure 13b indicates little or no functional relation between change in $\mathrm{L}-\mathrm{CV}$ and contributing drainage area. This lack of relation is consistent with previous L-CV graphs.

Changes in reservoir characteristics are estimated for an ungaged site by determining the maximum and normal capacity for current conditions and assuming that the reservoir characteristics are zero for the natural condition. For example, consider a hypothetical 1,000$\mathrm{mi}^{2}$ basin in the southeast region with a basin shape factor of 30, a slope of $10 \mathrm{ft} / \mathrm{mi}$, and a potential flood storage of about $200 \mathrm{acre}-\mathrm{ft} / \mathrm{mi}^{2}$ (current potential flood storage $=200,000$ acre-ft). The peak streamflow for such a basin might have been influenced by regulation on the basis of inspection of how $1,000 \mathrm{mi}^{2}$ and $200 \mathrm{acre}-\mathrm{ft} / \mathrm{mi}^{2}$ would plot in figure $6 \mathrm{c}$. For this example, it is assumed that consideration of regulation effects is warranted. From figure 11a, the applicable equation $\left(-4.98 \log _{10}[200]+7.98\right)$ produces a reduction in the mean of about 3.5 cubic feet per second per square mile $\left(\mathrm{ft}^{3} / \mathrm{s}-\mathrm{mi}^{2}\right)$.

\section{SUMMARY}

Estimates of peak-streamflow frequency are useful for flood-plain management, for objective assessment of flood risk, and for cost-effective design of hydraulic structures. Peak-streamflow frequency refers to peak streamflows that have recurrence intervals of 2 , $5,10,25,50,100,250$, and 500 years. Numerous techniques exist to estimate peak-streamflow frequency for gaged and ungaged stream sites in natural (unregulated) watersheds in Texas. However, such techniques are of questionable applicability for stream sites in watersheds affected by human-produced regulation, principally through the construction of dams. To address this situation, the USGS, in cooperation with TxDOT, has investigated flood characteristics in regulated basins in Texas.

Historically, a natural basin in Texas has been defined by the USGS as a basin with less than 10percent impervious cover, less than 10 percent of its drainage area controlled by reservoirs, and no other human-related factors that would affect peak streamflow. A regulated basin, therefore, was initially defined as a basin for which 10 percent or more of the drainage area of the basin is affected or controlled by reservoirs.

The State was divided into three regions. These regions (northeast, southeast, and west) were selected on the basis of regional boundaries used in a previous, related study, consideration of reservoir density, and density and locations of regulated streamflow-gaging stations. Four regression equations were derived for each region from data for 367 streamflow-gaging stations in basins designated as natural by the 10 percent criterion to estimate the L-moments of annual peak-streamflow data for ungaged sites. The stations used all have 10 or more years of natural annual peak-streamflow data through 1997 and have an average period of record of about 30 years. Four of the resulting 12 equations are constants. The independent variables in the equations are contributing drainage area, basin shape factor, and stream slope. A natural peak-streamflow frequency curve can be subsequently produced by fitting a distribution to the estimated natural L-moments.

The TNRCC database of dams was used to produce statistics reflecting the effects of regulation on peak streamflow. Some of the characteristics used in this investigation included number of the dam, name of the dam, reservoir name, latitude and longitude, county, purpose of the dam, year completed, maximum capacity, normal capacity, surface area, and drainage area. The total number of dams in the database exceeds 7,300. However, not all the data fields pertinent to this investigation were complete-latitude, longitude, and other important information sometimes were missing. Excluding dams with extensive missing data resulted in 7,035 dams used in the analysis. The statewide total maximum capacity is about 98.6 million acre- $\mathrm{ft}$, total normal capacity is about 41.5 million acre- $\mathrm{ft}$, and total surface area is about 1.25 million acres. Four variables were used to represent regulation in a basin. Three of the variables are maximum capacity, normal capacity, and surface area, the values of which were taken directly from the inventory of dams. The fourth variable, potential flood storage, was computed as the difference between maximum capacity and normal capacity. Potential flood storage is a hypothetical number and, therefore, is only a rough estimate of the total floodcontrolling capacity in a basin.

The USGS database of annual peak streamflow contains data from about 330 stations in basins designated as regulated by the 10 -percent criterion. Many stations have both natural and regulated record. About 
192 stations have 10 or more years of regulated record through 1997. The average period of record for these stations is about 28 years. For each of the 192 original regulated stations, a time series of cumulative changes in the maximum capacity, normal capacity, and surface area (cumulative changes in regulation) was generated. The stations that are most pertinent are those with periods of record that have two or more consecutive static or minimally changing periods of regulation. Of the 192 stations, 96 were identified as meeting this criterion. The mean period of regulated record for those 96 stations is about 34 years. A representative maximumcapacity and normal-capacity value for each station and for each static period was determined.

The effects of regulation were quantified by the changes in L-moments of annual peak streamflow for each available pair of consecutive static periods. The change in the L-moments of the streamflow were related to changes in reservoir characteristics (therefore, degree of regulation). For each of the regulated stations with two or more static periods of regulation, the L-moments of the streamflow for each static period were estimated. Subsequently, the change in the L-moments between two consecutive static periods as well as the change in reservoir characteristics were calculated. Also, the Mann-Kendall test was done on the annual peak-streamflow time series of each pair of consecutive static periods to indicate trends in annual peak streamflow with time.

Analysis of the relations between the L-moment and the variables representing regulation indicates that L-moments other than the mean are negligibly affected by regulation. The analysis indicated that as potential flood storage in a basin increases, the mean annual peak streamflow decreases nonlinearly.

\section{SELECTED REFERENCES}

Asquith, W.H., 1998a, Depth-duration frequency of precipitation for Texas: U.S. Geological Survey WaterResources Investigations Report 98-4044, 107 p., 3 app. 1998b, Peak-flow frequency for tributaries of the Colorado River downstream of Austin, Texas: U.S. Geological Survey Water-Resources Investigations Report 98-4015, 19 p., 1 app.

1999, Areal reduction factors for the 1-day design storm in Texas: U.S. Geological Survey WaterResources Investigations Report 99-4267, 81 p.

Asquith, W.H., and Famiglietti, J.S., 2000, Precipitation areal-reduction factor estimation using an annual- maxima centered approach: Journal of Hydrology, v. 230, p. 55-69.

Asquith, W.H., and Slade, R.M., Jr., 1995, Documented and potential extreme peak discharges and relation between potential extreme peak discharges and probable maximum flood peak discharges in Texas: U.S. Geological Survey Water-Resources Investigations Report 95-4249, 58 p.

1997, Regional equations for estimation of peakstreamflow frequency for natural basins in Texas: U.S. Geological Survey Water-Resources Investigations Report 96-4307, 68 p.

1999, Site-specific estimation of peak-streamflow frequency using generalized least-squares regression for natural basins in Texas: U.S. Geological Survey WaterResources Investigations Report 99-4172, 19 p.

Benson, M.A., 1962, Factors influencing the occurrence of floods in a humid region of diverse terrain: U.S. Geological Survey Water-Supply Paper 1580-B, 64 p.

1964, Factors influencing the occurrence of floods in the southwest: U.S. Geological Survey Water-Supply Paper 1580-D, 72 p.

David, H.A., 1981, Order statistics: New York, John Wiley, $360 \mathrm{p}$.

Helsel, D.R., and Hirsch, R.M., 1992, Studies in environmental science 49-Statistical methods in water resources: New York, Elsevier, 522 p.

Hollander M., and Wolfe, D.A., 1973, Nonparametric statistical methods: New York, John Wiley, 503 p.

Hosking, J.R.M., 1990, L-moments-Analysis and estimation of distributions using linear combinations of order statistics: Journal Royal Statistical Society B, v. 52, no. 1 , p. 105-124.

1994, The four-parameter kappa distribution: IBM Journal of Research and Development, v. 38, p. 251-258.

1996, Fortran routines for use with the method of L-moments, version 3: Yorktown Heights, N.Y., IBM Research Division, Research Report RC 20525.

Hosking, J.R.M., and Wallis, J.R., 1997, Regional frequency analysis-An approach based on L-moments: Cambridge University Press, 224 p.

Interagency Advisory Committee on Water Data, 1982, Guidelines for determining flood flow frequency: Reston, Va., U.S. Geological Survey, Office of Water Data Coordination, Hydrology Subcommittee Bulletin 17B [variously paged].

Judd, L.J., Asquith, W.H., and Slade, R.M., Jr., 1996, Techniques to estimate generalized skew coefficients of annual peak streamflow for natural basins in Texas: U.S. Geological Survey Water-Resources Investigations Report 96-4117, 28 p. 
Kirby, W.H., 1974, Algebraic boundness of sample statistics: Water Resources Research, v. 10, no. 2, p. 220-222.

Lanning-Rush, Jennifer, Asquith, W.H., and Slade, R.M., Jr., 1998, Extreme precipitation depths for Texas, excluding the Trans-Pecos region: U.S. Geological Survey WaterResources Investigations Report 98-4099, 38 p.

Slade, R.M., Jr., and Asquith, W.H., 1996, Peak data for U.S. Geological Survey gaging stations, Texas network; and computer program to estimate peak-streamflow frequency: U.S. Geological Survey Open-File Report 96-148, 57 p.
Statware, Inc., 1990, SREGRES in Statit statistics reference manual (release 2.3X): Corvallis, Oreg., Statware, Inc. [variously paged].

Stedinger, J.R., Vogel, R.M., and Foufoula-Georgiou, Efi, 1993, Frequency analysis of extreme events, in Maidment, D.A., ed., Handbook of applied hydrology, chap. 18: New York, McGraw-Hill, p. 18.1-66.

Vogel, R.M., and Fennessey, N.M., 1993, L-moment diagrams should replace product moment diagrams: Water Resources Research, v. 29, no. 6, p. 1,745-1,752.

Wallis, J.R., Matalas, N.C., and Slack, J.R., 1974, Just a moment: Water Resources Research, v. 10, no. 2, p. 211-219. 
$\approx \quad$ Table 1. Streamflow statistics and basin characteristics for 367 USGS streamflow-gaging stations with at least 10 years of annual peak-streamflow data through 1997 (designated as natural by 10-percent criterion)

Seq., sequence; $\mathrm{mi}^{2}$, square miles; $\mathrm{ft} / \mathrm{mi}$, feet per mile; $\mathrm{ft}^{3} / \mathrm{s}$, cubic feet per second; $\mathrm{W}$, west; NE, northeast; SE, southeast]

\begin{tabular}{|c|c|c|c|c|c|c|c|c|c|c|c|c|c|}
\hline \multirow{2}{*}{$\begin{array}{l}\text { Seq. } \\
\text { no. }\end{array}$} & \multirow{2}{*}{$\begin{array}{c}\text { USGS } \\
\text { station } \\
\text { no. } \\
\text { (pl. 1) }\end{array}$} & \multirow[b]{2}{*}{ USGS station name } & \multirow[b]{2}{*}{ Latitude } & \multirow[b]{2}{*}{ Longitude } & \multirow[b]{2}{*}{ Region } & \multirow{2}{*}{$\begin{array}{c}\text { Years } \\
\text { of } \\
\text { natural } \\
\text { annual } \\
\text { peak } \\
\text { stream- } \\
\text { flow }\end{array}$} & \multirow{2}{*}{$\begin{array}{c}\text { Contri- } \\
\text { buting } \\
\text { drainage } \\
\text { area } \\
\left(\mathrm{mi}^{2}\right)\end{array}$} & \multirow{2}{*}{$\begin{array}{c}\text { Basin } \\
\text { shape } \\
\text { factor } \\
\text { (dimen- } \\
\text { sion- } \\
\text { less) }\end{array}$} & \multirow{2}{*}{$\begin{array}{l}\text { Stream } \\
\text { slope } \\
(\mathrm{ft} / \mathrm{mi})\end{array}$} & \multicolumn{4}{|c|}{$\begin{array}{c}\text { L-moment statistics of annual } \\
\text { peak-streamflow data }\end{array}$} \\
\hline & & & & & & & & & & $\begin{array}{l}\text { Mean } \\
\left(\mathrm{ft}^{3} / \mathrm{s}\right)\end{array}$ & $\begin{array}{l}\text { L-scale } \\
\left(\mathrm{ft}^{3} / \mathrm{s}\right)\end{array}$ & L-skew & L-kurtosis \\
\hline 1 & 07227100 & Revuelto Creek near Logan, New Mexico & $35^{\circ} 20^{\prime} 24^{\prime \prime}$ & $103^{\circ} 23^{\prime} 24^{\prime \prime}$ & $\mathrm{W}$ & 38 & 786 & 5.07 & 13.20 & 7,701 & $3,095.4$ & 0.407 & 0.196 \\
\hline 2 & 07227920 & Dixon Creek near Borger, Texas & $35^{\circ} 39^{\prime} 53^{\prime \prime}$ & $101^{\circ} 21^{\prime} 02^{\prime \prime}$ & $\mathrm{W}$ & 15 & 134 & 5.56 & 23.55 & 1,486 & 687.8 & .145 & -.019 \\
\hline 3 & 07228000 & Canadian River near Canadian, Texas & $35^{\circ} 56^{\prime} 06^{\prime \prime}$ & $100^{\circ} 22^{\prime} 13^{\prime \prime}$ & $\mathrm{W}$ & 26 & 18,178 & 14.64 & 16.65 & 39,515 & $17,154.3$ & .263 & .053 \\
\hline 4 & 07233500 & Palo Duro Creek near Spearman, Texas & $36^{\circ} 12^{\prime} 08^{\prime \prime}$ & $101^{\circ} 18^{\prime} 20^{\prime \prime}$ & $\mathrm{W}$ & 35 & 440 & 13.98 & 10.17 & 3,711 & $2,024.7$ & .479 & .301 \\
\hline 5 & 07295500 & Tierra Blanca Creek above Buffalo Lake near Umbarger, Texas & $34^{\circ} 50^{\prime} 55^{\prime \prime}$ & $102^{\circ} 10^{\prime} 32^{\prime \prime}$ & $\mathrm{W}$ & 21 & 538 & 27.85 & 9.73 & 2,165 & $1,360.7$ & .507 & .345 \\
\hline 6 & 07297910 & Prairie Dog Town Fork Red River near Wayside, Texas & $34^{\circ} 50^{\prime} 15^{\prime \prime}$ & $101^{\circ} 24^{\prime} 49^{\prime \prime}$ & $\mathrm{W}$ & 30 & 930 & 47.93 & 11.24 & 14,104 & $7,304.1$ & .427 & .237 \\
\hline 7 & 07298000 & North Tule Draw at Reservoir near Tulia, Texas & $34^{\circ} 33^{\prime} 34^{\prime \prime}$ & $101^{\circ} 42^{\prime} 33^{\prime \prime}$ & $\mathrm{W}$ & 31 & 65 & 19.65 & 10.65 & 1,712 & $1,060.0$ & .476 & .281 \\
\hline 8 & 07298500 & Prairie Dog Town Fork Red River near Brice, Texas & $34^{\circ} 37^{\prime} 40^{\prime \prime}$ & $100^{\circ} 56^{\prime} 25^{\prime \prime}$ & $\mathrm{W}$ & 11 & 1,581 & 40.80 & 10.98 & 25,957 & $8,113.6$ & .133 & -.034 \\
\hline 9 & 07299200 & Prairie Dog Town Fork Red River near Lakeview, Texas & $34^{\circ} 34^{\prime} 23^{\prime \prime}$ & $100^{\circ} 44^{\prime} 43^{\prime \prime}$ & $\mathrm{W}$ & 16 & 2,023 & 35.33 & 10.87 & 27,975 & $11,231.7$ & .477 & .302 \\
\hline 10 & 07299300 & Little Red River near Turkey, Texas & $34^{\circ} 32^{\prime} 27^{\prime \prime}$ & $100^{\circ} 46^{\prime} 13^{\prime \prime}$ & $\mathrm{W}$ & 14 & 139 & 9.47 & 34.35 & 2,605 & 491.7 & -.154 & .108 \\
\hline 11 & 07299500 & Prairie Dog Town Fork Red River near Estelline, Texas & $34^{\circ} 30^{\prime} 20^{\prime \prime}$ & $100^{\circ} 26^{\prime} 10^{\prime \prime}$ & $\mathrm{W}$ & 12 & 2,524 & 33.12 & 10.67 & 26,730 & $9,412.4$ & .179 & .096 \\
\hline 12 & 07299540 & Prairie Dog Town Fork Red River near Childress, Texas & $34^{\circ} 34^{\prime} 09^{\prime \prime}$ & $100^{\circ} 11^{\prime} 37^{\prime \prime}$ & $\mathrm{W}$ & 14 & 2,956 & 32.13 & 10.38 & 31,103 & $14,529.1$ & .392 & .139 \\
\hline 13 & 07299570 & Red River near Quanah, Texas & $34^{\circ} 24^{\prime} 47^{\prime \prime}$ & $99^{\circ} 44^{\prime} 03^{\prime \prime}$ & $\mathrm{W}$ & 19 & 3,552 & 33.79 & 9.87 & 27,051 & $10,463.8$ & .296 & .171 \\
\hline 14 & 07299670 & Groesbeck Creek at SH 6 near Quanah, Texas & $34^{\circ} 21^{\prime} 16^{\prime \prime}$ & $99^{\circ} 44^{\prime} 24^{\prime \prime}$ & $\mathrm{W}$ & 36 & 303 & 5.84 & 10.22 & 3,613 & $2,097.8$ & .457 & .219 \\
\hline 15 & 07300000 & Salt Fork Red River near Wellington, Texas & $34^{\circ} 57^{\prime} 27^{\prime \prime}$ & $100^{\circ} 13^{\prime} 14^{\prime \prime}$ & $\mathrm{W}$ & 15 & 1,013 & 7.59 & 16.72 & 33,088 & $19,882.9$ & .522 & .314 \\
\hline 16 & 07300500 & Salt Fork Red River at Mangum, Oklahoma & $34^{\circ} 51^{\prime} 30^{\prime \prime}$ & $99^{\circ} 30^{\prime} 30^{\prime \prime}$ & $\mathrm{W}$ & 60 & 1,357 & 12.84 & 13.80 & 16,782 & $7,311.3$ & .324 & .164 \\
\hline 17 & 07301300 & North Fork Red River near Shamrock, Texas & $35^{\circ} 15^{\prime} 51^{\prime \prime}$ & $100^{\circ} 14^{\prime} 29^{\prime \prime}$ & $\mathrm{W}$ & 34 & 703 & 18.50 & 9.61 & 5,824 & $2,574.4$ & .392 & .295 \\
\hline 18 & 07301410 & Sweetwater Creek near Kelton, Texas & $35^{\circ} 28^{\prime} 23^{\prime \prime}$ & $100^{\circ} 07^{\prime} 14^{\prime \prime}$ & $\mathrm{W}$ & 36 & 267 & 7.66 & 15.89 & 701 & 303.7 & .294 & .234 \\
\hline 19 & 07307500 & Quitaque Creek near Quitaque, Texas & $34^{\circ} 14^{\prime} 24^{\prime \prime}$ & $101^{\circ} 07^{\prime} 03^{\prime \prime}$ & $\mathrm{W}$ & 14 & 35 & 17.25 & 25.10 & 1,539 & 785.1 & .534 & .404 \\
\hline 20 & 07307800 & Pease River near Childress, Texas & $34^{\circ} 13^{\prime} 39^{\prime \prime}$ & $100^{\circ} 04^{\prime} 24^{\prime \prime}$ & $\mathrm{W}$ & 33 & 2,195 & 5.35 & 16.22 & 8,427 & $3,158.9$ & .272 & .183 \\
\hline 21 & 07308000 & Pease River near Crowell, Texas & $34^{\circ} 05^{\prime} 45^{\prime \prime}$ & $99^{\circ} 43^{\prime} 47^{\prime \prime}$ & $\mathrm{W}$ & 23 & 2,478 & 8.02 & 13.66 & 44,217 & $14,584.6$ & .176 & .175 \\
\hline 22 & 07308200 & Pease River near Vernon, Texas & $34^{\circ} 10^{\prime} 44^{\prime \prime}$ & $99^{\circ} 16^{\prime} 40^{\prime \prime}$ & $\mathrm{W}$ & 36 & 2,929 & 10.97 & 11.60 & 13,122 & $5,534.3$ & .228 & .069 \\
\hline 23 & 07308500 & Red River near Burkburnett, Texas & $34^{\circ} 06^{\prime} 36^{\prime \prime}$ & $98^{\circ} 31^{\prime} 53^{\prime \prime}$ & $\mathrm{W}$ & 38 & 14,634 & 14.79 & 8.36 & 41,897 & $18,346.6$ & .401 & .283 \\
\hline 24 & 07311600 & North Wichita River near Paducah, Texas & $33^{\circ} 57^{\prime} 02^{\prime \prime}$ & $100^{\circ} 03^{\prime} 52^{\prime \prime}$ & $\mathrm{W}$ & 24 & 540 & 6.92 & 14.49 & 4,012 & $2,007.2$ & .360 & .235 \\
\hline 25 & 07311700 & North Wichita River near Truscott, Texas & $33^{\circ} 49^{\prime} 14^{\prime \prime}$ & $99^{\circ} 47^{\prime} 10^{\prime \prime}$ & $\mathrm{W}$ & 37 & 937 & 9.82 & 11.12 & 6,783 & $3,354.0$ & .453 & .280 \\
\hline 26 & 07311800 & South Fork Wichita River near Benjamin, Texas & $33^{\circ} 38^{\prime} 39^{\prime \prime}$ & $99^{\circ} 48^{\prime} 02^{\prime \prime}$ & $\mathrm{W}$ & 37 & 584 & 18.24 & 9.63 & 4,534 & $1,943.5$ & .429 & .166 \\
\hline 27 & 07311900 & Wichita River near Seymour, Texas & $33^{\circ} 42^{\prime} 01^{\prime \prime}$ & $99^{\circ} 23^{\prime} 18^{\prime \prime}$ & $\mathrm{W}$ & 20 & 1,874 & 12.85 & 7.57 & 10,290 & $2,990.8$ & .220 & .154 \\
\hline
\end{tabular}


Table 1. Streamflow statistics and basin characteristics for 367 USGS streamflow-gaging stations with at least 10 years of annual peak-streamflow data through 1997 (designated as natural by 10-percent criterion)—Continued

\begin{tabular}{|c|c|c|c|c|c|c|c|c|c|c|c|c|c|}
\hline \multirow{2}{*}{$\begin{array}{l}\text { Seq. } \\
\text { no. }\end{array}$} & \multirow{2}{*}{$\begin{array}{l}\text { USGS } \\
\text { station } \\
\text { no. } \\
\text { (pl. 1) }\end{array}$} & \multirow{2}{*}{ USGS station name } & \multirow{2}{*}{ Latitude } & \multirow{2}{*}{ Longitude } & \multirow{2}{*}{ Region } & \multirow{2}{*}{$\begin{array}{c}\text { Years } \\
\text { of } \\
\text { natural } \\
\text { annual } \\
\text { peak } \\
\text { stream- } \\
\text { flow }\end{array}$} & \multirow{2}{*}{$\begin{array}{c}\text { Contri- } \\
\text { buting } \\
\text { drainage } \\
\text { area } \\
\left(\mathrm{mi}^{2}\right)\end{array}$} & \multirow{2}{*}{$\begin{array}{c}\text { Basin } \\
\text { shape } \\
\text { factor } \\
\text { (dimen- } \\
\text { sion- } \\
\text { less) }\end{array}$} & \multirow{2}{*}{$\begin{array}{c}\text { Stream } \\
\text { slope } \\
\text { (ft/mi) }\end{array}$} & \multicolumn{4}{|c|}{$\begin{array}{l}\text { L-moment statistics of annual } \\
\text { peak-streamflow data }\end{array}$} \\
\hline & & & & & & & & & & $\begin{array}{l}\text { Mean } \\
\left(\mathrm{ft}^{3} / \mathrm{s}\right)\end{array}$ & $\begin{array}{l}\text { L-scale } \\
\left(\mathrm{ft}^{3} / \mathrm{s}\right)\end{array}$ & L-skew & L-kurtosis \\
\hline 28 & 07314500 & Little Wichita River near Archer City, Texas & $33^{\circ} 39^{\prime} 45^{\prime \prime}$ & $98^{\circ} 36^{\prime} 46^{\prime \prime}$ & $\mathrm{W}$ & 15 & 481 & 12.68 & 5.42 & 4,687 & $2,296.3$ & 0.562 & 0.345 \\
\hline 29 & 07315200 & East Fork Little Wichita River near Henrietta, Texas & $33^{\circ} 48^{\prime} 46^{\prime \prime}$ & $98^{\circ} 05^{\prime} 05^{\prime \prime}$ & $\mathrm{NE}$ & 30 & 178 & 5.51 & 8.90 & 4,683 & $3,164.9$ & .575 & .294 \\
\hline 30 & 07332600 & Bois d'Arc Creek near Randolph, Texas & $33^{\circ} 28^{\prime} 32^{\prime \prime}$ & $96^{\circ} 12^{\prime} 52^{\prime \prime}$ & $\mathrm{NE}$ & 23 & 72 & 4.49 & 10.41 & 9,057 & $2,412.0$ & -.016 & .177 \\
\hline 31 & 07335500 & Red River at Arthur City, Texas & $33^{\circ} 52^{\prime} 30^{\prime \prime}$ & $95^{\circ} 30^{\prime} 06^{\prime \prime}$ & $\mathrm{NE}$ & 13 & 38,595 & 10.39 & 7.03 & 119,823 & $54,530.8$ & .443 & .230 \\
\hline 32 & 07336750 & Little Pine Creek near Kanawha, Texas & $33^{\circ} 50^{\prime} 26^{\prime \prime}$ & $95^{\circ} 15^{\prime} 55^{\prime \prime}$ & $\mathrm{NE}$ & 12 & 75.4 & 5.10 & 6.53 & 8,466 & $3,951.3$ & .473 & .390 \\
\hline 33 & 07336800 & Pecan Bayou near Clarksville, Texas & $33^{\circ} 41^{\prime} 07^{\prime \prime}$ & $94^{\circ} 59^{\prime} 41^{\prime \prime}$ & $\mathrm{NE}$ & 16 & 100 & 7.57 & 5.48 & 5,352 & $2,239.0$ & .378 & .428 \\
\hline 34 & 07342470 & South Sulphur River near Commerce, Texas & $33^{\circ} 13^{\prime} 11^{\prime \prime}$ & $95^{\circ} 51^{\prime} 45^{\prime \prime}$ & $\mathrm{NE}$ & 12 & 189 & 6.10 & 6.57 & 11,743 & $2,628.2$ & .353 & .527 \\
\hline 35 & 07342500 & South Sulphur River near Cooper, Texas & $33^{\circ} 21^{\prime} 20^{\prime \prime}$ & $95^{\circ} 35^{\prime} 39^{\prime \prime}$ & $\mathrm{NE}$ & 49 & 527 & 8.00 & 4.75 & 15,849 & $5,116.9$ & .236 & .155 \\
\hline 36 & 07343000 & North Sulphur River near Cooper, Texas & $33^{\circ} 28^{\prime} 29^{\prime \prime}$ & $95^{\circ} 35^{\prime} 15^{\prime \prime}$ & $\mathrm{NE}$ & 48 & 276 & 6.18 & 6.83 & 36,351 & $8,644.3$ & .091 & .246 \\
\hline 37 & 07343200 & Sulphur River near Talco, Texas & $33^{\circ} 23^{\prime} 10^{\prime \prime}$ & $95^{\circ} 07 ' 56^{\prime \prime}$ & $\mathrm{NE}$ & 35 & 1,365 & 8.75 & 3.54 & 33,231 & $8,605.5$ & .142 & .134 \\
\hline 38 & 07343300 & Cuthand Creek near Bogata, Texas & $33^{\circ} 32^{\prime} 51^{\prime \prime}$ & $95^{\circ} 10^{\prime} 22^{\prime \prime}$ & $\mathrm{NE}$ & 11 & 69 & 6.07 & 7.85 & 6,306 & $2,716.4$ & .450 & .409 \\
\hline 39 & 07343500 & White Oak Creek near Talco, Texas & $33^{\circ} 19^{\prime} 20^{\prime \prime}$ & $95^{\circ} 05^{\prime} 33^{\prime \prime}$ & $\mathrm{NE}$ & 23 & 494 & 9.65 & 3.94 & 15,002 & $6,255.3$ & .266 & .151 \\
\hline 40 & 07343800 & White Oak Creek below Talco, Texas & $33^{\circ} 18^{\prime} 00^{\prime \prime}$ & $95^{\circ} 01^{\prime} 00^{\prime \prime}$ & $\mathrm{NE}$ & 13 & 579 & 9.97 & 3.76 & 27,737 & $9,279.7$ & .395 & .463 \\
\hline 41 & 07344000 & Sulphur River near Darden, Texas & $33^{\circ} 15^{\prime} 00^{\prime \prime}$ & $94^{\circ} 37^{\prime} 00^{\prime \prime}$ & $\mathrm{NE}$ & 47 & 2,774 & 11.12 & 2.63 & 35,289 & $13,706.1$ & .260 & .181 \\
\hline 42 & 07344486 & Brushy Creek at Scroggins, Texas & $32^{\circ} 58^{\prime} 32^{\prime \prime}$ & $95^{\circ} 11^{\prime} 03^{\prime \prime}$ & $\mathrm{NE}$ & 19 & 23.4 & 1.77 & 24.49 & 2,529 & $1,334.7$ & .436 & .287 \\
\hline 43 & 07344500 & Big Cypress Creek near Pittsburg, Texas & $33^{\circ} 01^{\prime} 15^{\prime \prime}$ & $94^{\circ} 52^{\prime} 55^{\prime \prime}$ & $\mathrm{NE}$ & 22 & 366 & 3.70 & 6.87 & 11,815 & $5,959.4$ & .480 & .336 \\
\hline 44 & 07345000 & Boggy Creek near Daingerfield, Texas & $33^{\circ} 02^{\prime} 10^{\prime \prime}$ & $94^{\circ} 47^{\prime} 15^{\prime \prime}$ & $\mathrm{NE}$ & 34 & 72 & 4.25 & 6.94 & 4,458 & $2,409.3$ & .527 & .370 \\
\hline 45 & 07346000 & Big Cypress Creek near Jefferson, Texas & $32^{\circ} 44^{\prime} 58^{\prime \prime}$ & $94^{\circ} 29^{\prime} 55^{\prime \prime}$ & $\mathrm{NE}$ & 45 & 850 & 6.88 & 4.29 & 10,413 & $5,390.5$ & .428 & .238 \\
\hline 46 & 07346045 & Black Cypress Bayou at Jefferson, Texas & $32^{\circ} 46^{\prime} 40^{\prime \prime}$ & $94^{\circ} 21^{\prime 2} 26^{\prime \prime}$ & $\mathrm{NE}$ & 29 & 365 & 7.80 & 3.75 & 4,128 & $1,632.4$ & .228 & .152 \\
\hline 47 & 07346050 & Little Cypress Creek near Ore City, Texas & $32^{\circ} 40^{\prime} 21^{\prime \prime}$ & $94^{\circ} 45^{\prime} 03^{\prime \prime}$ & $\mathrm{NE}$ & 35 & 383 & 5.96 & 4.47 & 5,688 & $2,721.5$ & .331 & .165 \\
\hline 48 & 07346070 & Little Cypress Creek near Jefferson, Texas & $32^{\circ} 42^{\prime} 50^{\prime \prime}$ & $94^{\circ} 20^{\prime} 44^{\prime \prime}$ & $\mathrm{NE}$ & 52 & 675 & 10.84 & 3.14 & 6,439 & $3,075.3$ & .407 & .250 \\
\hline 49 & 07346140 & Frazier Creek near Linden, Texas & $33^{\circ} 03^{\prime} 14^{\prime \prime}$ & $94^{\circ} 17^{\prime} 24^{\prime \prime}$ & $\mathrm{NE}$ & 27 & 48 & 2.62 & 10.53 & 2,123 & $1,075.5$ & .321 & .100 \\
\hline 50 & 08017200 & Cowleech Fork Sabine River at Greenville, Texas & $33^{\circ} 07^{\prime} 58^{\prime \prime}$ & $96^{\circ} 04^{\prime} 36^{\prime \prime}$ & $\mathrm{NE}$ & 39 & 77.7 & 4.66 & 8.96 & 5,828 & $1,621.5$ & .203 & .284 \\
\hline 51 & 08017300 & South Fork Sabine River near Quinlan, Texas & $32^{\circ} 53^{\prime} 52^{\prime \prime}$ & $96^{\circ} 15^{\prime} 11^{\prime \prime}$ & $\mathrm{NE}$ & 39 & 78.7 & 4.34 & 8.88 & 10,379 & $3,532.5$ & .087 & .044 \\
\hline 52 & 08018500 & Sabine River near Mineola, Texas & $32^{\circ} 36^{\prime} 49^{\prime \prime}$ & $95^{\circ} 29^{\prime} 08^{\prime \prime}$ & $\mathrm{NE}$ & 22 & 1,357 & 7.82 & 3.47 & 27,824 & $11,535.7$ & .156 & .074 \\
\hline 53 & 08018730 & Burke Creek near Yantis, Texas & $32^{\circ} 59^{\prime} 26^{\prime \prime}$ & $95^{\circ} 37^{\prime} 18^{\prime \prime}$ & $\mathrm{NE}$ & 11 & 33.1 & 2.88 & 10.41 & 2,875 & 715.0 & .101 & .473 \\
\hline 54 & 08019000 & Lake Fork Creek near Quitman, Texas & $32^{\circ} 45^{\prime} 47^{\prime \prime}$ & $95^{\circ} 27^{\prime} 46^{\prime \prime}$ & $\mathrm{NE}$ & 42 & 585 & 4.28 & 4.92 & 16,189 & $7,212.2$ & .351 & .248 \\
\hline 55 & 08019500 & Big Sandy Creek near Big Sandy, Texas & $32^{\circ} 36^{\prime} 14^{\prime \prime}$ & $95^{\circ} 05^{\prime} 29^{\prime \prime}$ & $\mathrm{NE}$ & 24 & 231 & 9.06 & 5.81 & 4,324 & $2,204.7$ & .490 & .361 \\
\hline 56 & 08020000 & Sabine River near Gladewater, Texas & $32^{\circ} 31^{\prime} 37^{\prime \prime}$ & $94^{\circ} 57^{\prime} 36^{\prime \prime}$ & $\mathrm{NE}$ & 29 & 2,791 & 9.39 & 2.57 & 28,418 & $14,492.2$ & .352 & .125 \\
\hline
\end{tabular}


$\omega$ Table 1. Streamflow statistics and basin characteristics for 367 USGS streamflow-gaging stations with at least 10 years of annual peak-streamflow data through 1997 (designated as natural by 10-percent criterion)-Continued

\begin{tabular}{|c|c|c|c|c|c|c|c|c|c|c|c|c|c|}
\hline \multirow{2}{*}{$\begin{array}{l}\text { Seq. } \\
\text { no. }\end{array}$} & \multirow{2}{*}{$\begin{array}{c}\text { USGS } \\
\text { station } \\
\text { no. } \\
\text { (pl. 1) }\end{array}$} & \multirow{2}{*}{ USGS station name } & \multirow{2}{*}{ Latitude } & \multirow{2}{*}{ Longitude } & \multirow{2}{*}{ Region } & \multirow{2}{*}{$\begin{array}{l}\text { Years } \\
\text { of } \\
\text { natural } \\
\text { annual } \\
\text { peak } \\
\text { stream- } \\
\text { flow }\end{array}$} & \multirow{2}{*}{$\begin{array}{l}\text { Contri- } \\
\text { buting } \\
\text { drainage } \\
\text { area } \\
\left(\mathrm{mi}^{2}\right)\end{array}$} & \multirow{2}{*}{$\begin{array}{c}\text { Basin } \\
\text { shape } \\
\text { factor } \\
\text { (dimen- } \\
\text { sion- } \\
\text { less) }\end{array}$} & \multirow{2}{*}{$\begin{array}{c}\text { Stream } \\
\text { slope } \\
\text { (ft/mi) }\end{array}$} & \multicolumn{4}{|c|}{$\begin{array}{l}\text { L-moment statistics of annual } \\
\text { peak-streamflow data }\end{array}$} \\
\hline & & & & & & & & & & $\begin{array}{l}\text { Mean } \\
\left(\mathrm{ft}^{3} / \mathrm{s}\right)\end{array}$ & $\begin{array}{l}\text { L-scale } \\
\left(\mathrm{ft}^{3} / \mathrm{s}\right)\end{array}$ & L-skew & L-kurtosis \\
\hline 57 & 08020500 & Sabine River near Longview, Texas & $32^{\circ} 28^{\prime} 00^{\prime \prime}$ & $94^{\circ} 46^{\prime} 50^{\prime \prime}$ & $\mathrm{NE}$ & 11 & 2,947 & 11.76 & 2.29 & 13,335 & $4,034.2$ & -0.031 & -0.107 \\
\hline 58 & 08020700 & Rabbit Creek at Kilgore, Texas & $32^{\circ} 23^{\prime} 17^{\prime \prime}$ & $94^{\circ} 54^{\prime} 11^{\prime \prime}$ & $\mathrm{NE}$ & 13 & 75.8 & 2.55 & 13.91 & 3,566 & $1,921.6$ & .427 & .285 \\
\hline 59 & 08022000 & Sabine River near Tatum, Texas & $32^{\circ} 22^{\prime} 11^{\prime \prime}$ & $94^{\circ} 27^{\prime} 28^{\prime \prime}$ & $\mathrm{NE}$ & 22 & 3,493 & 13.85 & 2.04 & 30,082 & $14,522.4$ & .376 & .182 \\
\hline 60 & 08022400 & Socagee Creek near Carthage, Texas & $32^{\circ} 13^{\prime} 54^{\prime \prime}$ & $94^{\circ} 05^{\prime} 31^{\prime \prime}$ & $\mathrm{NE}$ & 12 & 82.6 & 4.00 & 6.86 & 2,266 & $1,058.0$ & .422 & .259 \\
\hline 61 & 08022500 & Sabine River at Logansport, Louisiana & $31^{\circ} 58^{\prime} 20^{\prime \prime}$ & $94^{\circ} 00^{\prime} 22^{\prime \prime}$ & $\mathrm{NE}$ & 57 & 4,842 & 16.90 & 1.65 & 22,596 & $8,520.2$ & .281 & .115 \\
\hline 62 & 08023200 & Tenaha Creek near Shelbyville, Texas & $31^{\circ} 45^{\prime} 56^{\prime \prime}$ & $94^{\circ} 05^{\prime} 02^{\prime \prime}$ & $\mathrm{NE}$ & 30 & 97.8 & 3.96 & 9.00 & 4,443 & $2,293.2$ & .456 & .288 \\
\hline 63 & 08024400 & Sabine River near Milam, Texas & $31^{\circ} 28^{\prime} 01^{\prime \prime}$ & $93^{\circ} 44^{\prime} 41^{\prime \prime}$ & $\mathrm{NE}$ & 23 & 6,508 & 16.68 & 1.48 & 31,036 & $11,070.3$ & .336 & .107 \\
\hline 64 & 08024500 & Palo Gaucho Bayou near Hemphill, Texas & $31^{\circ} 23^{\prime} 10^{\prime \prime}$ & $93^{\circ} 50^{\prime} 08^{\prime \prime}$ & $\mathrm{NE}$ & 14 & 123 & 4.46 & 11.76 & 3,670 & $1,967.0$ & .527 & .349 \\
\hline 65 & 08028500 & Sabine River near Bon Weir, Texas & $30^{\circ} 44^{\prime} 49^{\prime \prime}$ & $93^{\circ} 36^{\prime} 30^{\prime \prime}$ & SE & 41 & 8,229 & 19.81 & 1.50 & 37,132 & $10,493.2$ & .312 & .213 \\
\hline 66 & 08029500 & Big Cow Creek near Newton, Texas & $30^{\circ} 49^{\prime} 08^{\prime \prime}$ & $93^{\circ} 47^{\prime} 07^{\prime \prime}$ & $\mathrm{SE}$ & 46 & 128 & 5.22 & 12.06 & 4,072 & $1,931.4$ & .469 & .254 \\
\hline 67 & 08030000 & Cypress Creek near Buna, Texas & $30^{\circ} 25^{\prime} 52^{\prime \prime}$ & $93^{\circ} 54^{\prime} 28^{\prime \prime}$ & $\mathrm{SE}$ & 32 & 69.2 & 4.81 & 4.50 & 2,544 & 836.6 & .303 & .256 \\
\hline 68 & 08030500 & Sabine River near Ruliff, Texas & $30^{\circ} 18^{\prime} 13^{\prime \prime}$ & $93^{\circ} 44^{\prime} 37^{\prime \prime}$ & SE & 54 & 9,329 & 22.74 & 1.43 & 42,033 & $11,556.7$ & .232 & .134 \\
\hline 69 & 08031000 & Cow Bayou near Mauriceville, Texas & $30^{\circ} 11^{\prime} 10^{\prime \prime}$ & $93^{\circ} 54^{\prime} 30^{\prime \prime}$ & $\mathrm{SE}$ & 34 & 83.3 & 7.62 & 2.60 & 1,701 & 613.5 & .337 & .167 \\
\hline 70 & 08031200 & Kickapoo Creek near Brownsboro, Texas & $32^{\circ} 18^{\prime} 34^{\prime \prime}$ & $95^{\circ} 36^{\prime} 19^{\prime \prime}$ & $\mathrm{NE}$ & 28 & 232 & 5.44 & 4.34 & 4,568 & $2,066.2$ & .328 & .193 \\
\hline 71 & 08032000 & Neches River near Neches, Texas & $31^{\circ} 53^{\prime} 32^{\prime \prime}$ & $95^{\circ} 25^{\prime} 50^{\prime \prime}$ & $\mathrm{NE}$ & 23 & 1,145 & 6.06 & 3.19 & 10,817 & $4,796.2$ & .342 & .246 \\
\hline 72 & 08032500 & Neches River near Alto, Texas & $31^{\circ} 34^{\prime} 45^{\prime \prime}$ & $95^{\circ} 09^{\prime} 55^{\prime \prime}$ & $\mathrm{NE}$ & 18 & 1,945 & 10.38 & 2.24 & 12,693 & $6,170.9$ & .365 & .118 \\
\hline 73 & 08033000 & Neches River near Diboll, Texas & $31^{\circ} 07^{\prime} 58^{\prime \prime}$ & $94^{\circ} 48^{\prime} 35^{\prime \prime}$ & $\mathrm{NE}$ & 23 & 2,724 & 17.07 & 1.87 & 14,510 & $6,501.0$ & .317 & .178 \\
\hline 74 & 08033300 & Piney Creek near Groveton, Texas & $31^{\circ} 08^{\prime} 25^{\prime \prime}$ & $95^{\circ} 05^{\prime} 11^{\prime \prime}$ & SE & 28 & 79 & 5.69 & 7.58 & 2,183 & $1,128.7$ & .289 & .008 \\
\hline 75 & 08033500 & Neches River near Rockland, Texas & $31^{\circ} 01^{\prime} 29^{\prime \prime}$ & $94^{\circ} 23^{\prime} 55^{\prime \prime}$ & SE & 27 & 3,636 & 18.83 & 1.71 & 17,880 & $6,950.8$ & .261 & .080 \\
\hline 76 & 08033900 & East Fork Angelina River near Cushing, Texas & $31^{\circ} 51^{\prime} 36^{\prime \prime}$ & $94^{\circ} 49^{\prime} 23^{\prime \prime}$ & $\mathrm{NE}$ & 26 & 158 & 4.69 & 7.47 & 5,463 & $2,738.9$ & .361 & .206 \\
\hline 77 & 08037000 & Angelina River near Lufkin, Texas & $31^{\circ} 27^{\prime} 26^{\prime \prime}$ & $94^{\circ} 43^{\prime} 34^{\prime \prime}$ & $\mathrm{NE}$ & 28 & 1,600 & 9.05 & 2.73 & 11,235 & $4,972.2$ & .343 & .187 \\
\hline 78 & 08037050 & Bayou Lanana at Nacogdoches, Texas & $31^{\circ} 36^{\prime} 58^{\prime \prime}$ & $94^{\circ} 38^{\prime} 28^{\prime \prime}$ & $\mathrm{NE}$ & 27 & 31.3 & 5.54 & 13.46 & 3,049 & $1,450.6$ & .397 & .272 \\
\hline 79 & 08038000 & Attoyac Bayou near Chireno, Texas & $31^{\circ} 30^{\prime} 15^{\prime \prime}$ & $94^{\circ} 18^{\prime} 15^{\prime \prime}$ & $\mathrm{NE}$ & 59 & 503 & 11.52 & 4.44 & 8,725 & $4,162.0$ & .285 & .110 \\
\hline 80 & 08038500 & Angelina River near Zavalla, Texas & $31^{\circ} 12^{\prime} 41^{\prime \prime}$ & $94^{\circ} 17^{\prime} 40^{\prime \prime}$ & $\mathrm{NE}$ & 14 & 2,892 & 8.77 & 2.06 & 12,439 & $5,885.0$ & .389 & .186 \\
\hline 81 & 08039100 & Ayish Bayou near San Augustine, Texas & $31^{\circ} 23^{\prime} 46^{\prime \prime}$ & $94^{\circ} 09^{\prime} 03^{\prime \prime}$ & $\mathrm{NE}$ & 40 & 89 & 5.53 & 10.06 & 5,093 & $2,228.6$ & .318 & .206 \\
\hline 82 & 08039500 & Angelina River at Horger, Texas & $31^{\circ} 02^{\prime} 08^{\prime \prime}$ & $94^{\circ} 07^{\prime} 48^{\prime \prime}$ & SE & 30 & 3,486 & 9.74 & 2.35 & 19,032 & $6,369.9$ & .303 & .131 \\
\hline 83 & 08041000 & Neches River at Evadale, Texas & $30^{\circ} 21^{\prime} 20^{\prime \prime}$ & $94^{\circ} 05^{\prime} 35^{\prime \prime}$ & $\mathrm{SE}$ & 32 & 7,951 & 16.17 & 1.45 & 37,964 & $12,739.7$ & .236 & .066 \\
\hline 84 & 08041500 & Village Creek near Kountze, Texas & $30^{\circ} 23^{\prime} 52^{\prime \prime}$ & $94^{\circ} 15^{\prime} 48^{\prime \prime}$ & SE & 61 & 860 & 7.18 & 4.47 & 14,340 & $6,991.2$ & .426 & .223 \\
\hline 85 & 08041700 & Pine Island Bayou near Sour Lake, Texas & $30^{\circ} 06^{\prime} 21^{\prime \prime}$ & $94^{\circ} 20^{\prime} 04^{\prime \prime}$ & SE & 30 & 336 & 8.95 & 2.27 & 7,223 & $3,761.9$ & .597 & .459 \\
\hline
\end{tabular}


Table 1. Streamflow statistics and basin characteristics for 367 USGS streamflow-gaging stations with at least 10 years of annual peak-streamflow data through 1997 (designated as natural by 10-percent criterion)—Continued

\begin{tabular}{|c|c|c|c|c|c|c|c|c|c|c|c|c|c|}
\hline \multirow{2}{*}{$\begin{array}{l}\text { Seq. } \\
\text { no. }\end{array}$} & \multirow{2}{*}{$\begin{array}{c}\text { USGS } \\
\text { station } \\
\text { no. } \\
\text { (pl. 1) }\end{array}$} & \multirow{2}{*}{ USGS station name } & \multirow{2}{*}{ Latitude } & \multirow{2}{*}{ Longitude } & \multirow{2}{*}{ Region } & \multirow{3}{*}{$\begin{array}{c}\begin{array}{c}\text { Years } \\
\text { of } \\
\text { natural } \\
\text { annual } \\
\text { peak } \\
\text { stream- } \\
\text { flow }\end{array} \\
30\end{array}$} & \multirow{3}{*}{$\begin{array}{c}\begin{array}{c}\text { Contri- } \\
\text { buting } \\
\text { drainage } \\
\text { area } \\
\left(\mathbf{m i}^{2}\right)\end{array} \\
262\end{array}$} & \multirow{3}{*}{$\begin{array}{r}\begin{array}{c}\text { Basin } \\
\text { shape } \\
\text { factor } \\
\text { (dimen- } \\
\text { sion- } \\
\text { less) }\end{array} \\
2.49\end{array}$} & \multirow{2}{*}{$\begin{array}{l}\text { Stream } \\
\text { slope } \\
(\mathrm{ft} / \mathrm{mi})\end{array}$} & \multicolumn{4}{|c|}{$\begin{array}{l}\text { L-moment statistics of annual } \\
\text { peak-streamflow data }\end{array}$} \\
\hline & & & & & & & & & & $\begin{array}{l}\text { Mean } \\
\left(\mathrm{ft}^{3} / \mathrm{s}\right)\end{array}$ & $\begin{array}{c}\text { L-scale } \\
\left(\mathrm{ft}^{3} / \mathbf{s}\right)\end{array}$ & L-skew & L-kurtosis \\
\hline 86 & 08042000 & Taylor Bayou near Labelle, Texas & $29^{\circ} 52^{\prime} 30^{\prime \prime}$ & $94^{\circ} 09^{\prime} 34^{\prime \prime}$ & SE & & & & 1.22 & 5,849 & 851.8 & 0.032 & 0.222 \\
\hline 87 & 08042500 & Hillebrandt Bayou near Lovell Lake, Texas & $29^{\circ} 55^{\prime} 44^{\prime \prime}$ & $94^{\circ} 06^{\prime} 35^{\prime \prime}$ & SE & 30 & 128 & 2.25 & 1.55 & 6,060 & $1,523.2$ & .125 & .191 \\
\hline 88 & 08042700 & North Creek near Jacksboro, Texas & $33^{\circ} 16^{\prime} 57^{\prime \prime}$ & $98^{\circ} 17^{\prime} 53^{\prime \prime}$ & $\mathrm{NE}$ & 18 & 21.6 & 5.39 & 27.97 & 2,527 & $1,009.9$ & .265 & .183 \\
\hline 89 & 08042800 & West Fork Trinity River near Jacksboro, Texas & $33^{\circ} 17^{\prime} 36^{\prime \prime}$ & $98^{\circ} 04^{\prime} 43^{\prime \prime}$ & $\mathrm{NE}$ & 19 & 683 & 5.83 & 5.35 & 6,272 & $3,808.0$ & .609 & .378 \\
\hline 90 & 08043500 & West Fork Trinity River at Bridgeport, Texas & $33^{\circ} 12^{\prime} 05^{\prime \prime}$ & $97^{\circ} 45^{\prime} 21^{\prime \prime}$ & $\mathrm{NE}$ & 25 & 1147 & 9.70 & 4.54 & 8,300 & $2,578.6$ & .215 & .151 \\
\hline 91 & 08044000 & Big Sandy Creek near Bridgeport, Texas & $33^{\circ} 13^{\prime} 54 "$ & $97^{\circ} 41^{\prime} 40^{\prime \prime}$ & $\mathrm{NE}$ & 19 & 333 & 7.42 & 8.18 & 7,253 & $4,758.2$ & .646 & .543 \\
\hline 92 & 08047500 & Clear Fork Trinity River at Fort Worth, Texas & $32^{\circ} 43^{\prime} 56^{\prime \prime}$ & $97^{\circ} 21 ' 31^{\prime \prime}$ & $\mathrm{NE}$ & 29 & 518 & 6.96 & 11.20 & 13,513 & $6,676.2$ & .515 & .442 \\
\hline 93 & 08048000 & West Fork Trinity River at Fort Worth, Texas & $32^{\circ} 45^{\prime} 39^{\prime \prime}$ & $97^{\circ} 19^{\prime} 56^{\prime \prime}$ & $\mathrm{NE}$ & 11 & 2,615 & 11.36 & 4.11 & 16,352 & $8,733.6$ & .731 & .718 \\
\hline 94 & 08049550 & Big Bear Creek near Grapevine, Texas & $32^{\circ} 54^{\prime} 48^{\prime \prime}$ & $97^{\circ} 07^{\prime} 44^{\prime \prime}$ & $\mathrm{NE}$ & 13 & 29.6 & 7.48 & 16.76 & 1,386 & 485.3 & .026 & .083 \\
\hline 95 & 08049580 & Mountain Creek near Venus, Texas & $32^{\circ} 39^{\prime} 07^{\prime \prime}$ & $96^{\circ} 59^{\prime} 24 "$ & $\mathrm{NE}$ & 12 & 25.5 & 36.62 & 12.24 & 3,814 & $1,918.3$ & .287 & .017 \\
\hline 96 & 08049700 & Walnut Creek near Mansfield, Texas & $32^{\circ} 34^{\prime} 51^{\prime \prime}$ & $97^{\circ} 06^{\prime} 06^{\prime \prime}$ & $\mathrm{NE}$ & 37 & 62.8 & 5.71 & 15.07 & 4,372 & $1,976.4$ & .351 & .246 \\
\hline 97 & 08050200 & Elm Fork Trinity Subwatershed No. 6-0 near Muenster, Texas & $33^{\circ} 37^{\prime} 13^{\prime \prime}$ & $97^{\circ} 24^{\prime} 15^{\prime \prime}$ & $\mathrm{NE}$ & 17 & .77 & .18 & 53.20 & 334 & 118.7 & .190 & .182 \\
\hline 98 & 08050400 & Elm Fork Trinity River at Gainesville, Texas & $33^{\circ} 27^{\prime} 27^{\prime \prime}$ & $97^{\circ} 09^{\prime} 22^{\prime \prime}$ & $\mathrm{NE}$ & 12 & 174 & 1.66 & 22.19 & 13,128 & $3,982.7$ & .141 & -.070 \\
\hline 99 & 08050800 & Timber Creek near Collinsville, Texas & $33^{\circ} 33^{\prime} 16^{\prime \prime}$ & $96^{\circ} 56^{\prime} 49^{\prime \prime}$ & $\mathrm{NE}$ & 12 & 38.8 & 5.23 & 13.13 & 4,172 & $2,254.3$ & .326 & .021 \\
\hline 100 & 08051000 & Isle du Bois Creek near Pilot Point, Texas & $33^{\circ} 24^{\prime} 23^{\prime \prime}$ & $97^{\circ} 00^{\prime} 45^{\prime \prime}$ & $\mathrm{NE}$ & 37 & 266 & 3.73 & 8.34 & 9,885 & $5,134.3$ & .404 & .178 \\
\hline 101 & 08051500 & Clear Creek near Sanger, Texas & $33^{\circ} 20^{\prime} 21^{\prime \prime}$ & $97^{\circ} 10^{\prime} 51^{\prime \prime}$ & $\mathrm{NE}$ & 32 & 295 & 7.99 & 10.13 & 7,408 & $3,103.9$ & .130 & .048 \\
\hline 102 & 08052630 & Little Elm Creek Subwatershed No. 10 near Gunter, Texas & $33^{\circ} 24^{\prime} 33^{\prime \prime}$ & $96^{\circ} 48^{\prime} 41^{\prime \prime}$ & $\mathrm{NE}$ & 11 & 2.10 & 3.12 & 39.74 & 1,332 & 485.6 & .176 & .261 \\
\hline 103 & 08053500 & Denton Creek near Justin, Texas & $33^{\circ} 07^{\prime} 08^{\prime \prime}$ & $97^{\circ} 17^{\prime} 25^{\prime \prime}$ & $\mathrm{NE}$ & 14 & 400 & 9.72 & 8.41 & 7,712 & $4,534.3$ & .430 & .134 \\
\hline 104 & 08054000 & Denton Creek near Roanoke, Texas & $33^{\circ} 02^{\prime} 24^{\prime \prime}$ & $97^{\circ} 12^{\prime} 17^{\prime \prime}$ & $\mathrm{NE}$ & 21 & 621 & 9.63 & 7.72 & 13,293 & $5,974.3$ & .423 & .278 \\
\hline 105 & 08055500 & Elm Fork Trinity River near Carrollton, Texas & $32^{\circ} 57^{\prime} 57^{\prime \prime}$ & $96^{\circ} 56^{\prime} 39^{\prime \prime}$ & $\mathrm{NE}$ & 28 & 2,459 & 4.22 & 5.25 & 27,565 & $12,953.9$ & .426 & .156 \\
\hline 106 & 08057000 & Trinity River at Dallas, Texas & $32^{\circ} 46^{\prime} 29^{\prime \prime}$ & $96^{\circ} 49^{\prime} 18^{\prime \prime}$ & $\mathrm{NE}$ & 30 & 6,106 & 8.37 & 3.66 & 30,225 & $14,851.7$ & .447 & .255 \\
\hline 107 & 08061500 & East Fork Trinity River near Rockwall, Texas & $32^{\circ} 55^{\prime} 25^{\prime \prime}$ & $96^{\circ} 30^{\prime} 20^{\prime \prime}$ & $\mathrm{NE}$ & 29 & 840 & 3.34 & 7.56 & 23,782 & $9,231.4$ & .189 & .080 \\
\hline 108 & 08061540 & Rowlett Creek near Sachse, Texas & $32^{\circ} 57^{\prime} 35^{\prime \prime}$ & $96^{\circ} 366^{\prime} 51^{\prime \prime}$ & $\mathrm{NE}$ & 29 & 120 & 4.63 & 12.11 & 12,370 & $5,073.4$ & .214 & .045 \\
\hline 109 & 08062500 & Trinity River near Rosser, Texas & $32^{\circ} 25^{\prime} 35^{\prime \prime}$ & $96^{\circ} 27^{\prime} 46^{\prime \prime}$ & $\mathrm{NE}$ & 14 & 8,146 & 9.17 & 3.31 & 34,514 & $10,838.5$ & .066 & -.042 \\
\hline 110 & 08063000 & Cedar Creek near Mabank, Texas & $32^{\circ} 19^{\prime} 45^{\prime \prime}$ & $96^{\circ} 10^{\prime} 05^{\prime \prime}$ & $\mathrm{NE}$ & 27 & 733 & 3.82 & 4.90 & 17,443 & $5,914.9$ & .149 & .058 \\
\hline 111 & 08063500 & Richland Creek near Richland, Texas & $31^{\circ} 57^{\prime} 02^{\prime \prime}$ & $96^{\circ} 25^{\prime} 16^{\prime \prime}$ & $\mathrm{NE}$ & 24 & 734 & 4.87 & 8.12 & 25,374 & $10,079.0$ & .130 & .031 \\
\hline 112 & 08064500 & Chambers Creek near Corsicana, Texas & $32^{\circ} 06^{\prime} 29^{\prime \prime}$ & $96^{\circ} 22^{\prime} 14^{\prime \prime}$ & $\mathrm{NE}$ & 22 & 963 & 7.66 & 6.26 & 18,955 & $6,989.6$ & .154 & .123 \\
\hline 113 & 08064700 & Tehuacana Creek near Streetman, Texas & $31^{\circ} 50^{\prime} 54^{\prime \prime}$ & $96^{\circ} 17^{\prime} 23^{\prime \prime}$ & $\mathrm{NE}$ & 30 & 142 & 4.08 & 10.77 & 13,866 & $7,820.9$ & .616 & .392 \\
\hline 114 & 08064800 & Catfish Creek near Tennessee Colony, Texas & $31^{\circ} 52^{\prime} 51^{\prime \prime}$ & $95^{\circ} 52^{\prime} 07^{\prime \prime}$ & $\mathrm{NE}$ & 28 & 207 & 4.71 & 6.72 & 1,846 & 933.2 & .473 & .299 \\
\hline
\end{tabular}


w Table 1. Streamflow statistics and basin characteristics for 367 USGS streamflow-gaging stations with at least 10 years of annual peak-streamflow data through 1997 (designated as natural by 10-percent criterion)-Continued

\begin{tabular}{|c|c|c|c|c|c|c|c|c|c|c|c|c|c|}
\hline \multirow{2}{*}{$\begin{array}{l}\text { Seq. } \\
\text { no. }\end{array}$} & \multirow{2}{*}{$\begin{array}{c}\text { USGS } \\
\text { station } \\
\text { no. } \\
\text { (pl. 1) }\end{array}$} & \multirow{2}{*}{ USGS station name } & \multirow{2}{*}{ Latitude } & \multirow{2}{*}{ Longitude } & \multirow{2}{*}{ Region } & \multirow{3}{*}{$\begin{array}{c}\begin{array}{c}\text { Years } \\
\text { of } \\
\text { natural } \\
\text { annual } \\
\text { peak } \\
\text { stream- } \\
\text { flow }\end{array} \\
29\end{array}$} & \multirow{3}{*}{$\begin{array}{c}\begin{array}{c}\text { Contri- } \\
\text { buting } \\
\text { drainage } \\
\text { area } \\
\left(\mathbf{m i}^{2}\right)\end{array} \\
12,833\end{array}$} & \multirow{3}{*}{$\begin{array}{c}\begin{array}{c}\text { Basin } \\
\text { shape } \\
\text { factor } \\
\text { (dimen- } \\
\text { sion- } \\
\text { less) }\end{array} \\
13.00\end{array}$} & \multirow{2}{*}{$\begin{array}{c}\text { Stream } \\
\text { slope } \\
(\mathrm{ft} / \mathrm{mi})\end{array}$} & \multicolumn{4}{|c|}{$\begin{array}{l}\text { L-moment statistics of annual } \\
\text { peak-streamflow data }\end{array}$} \\
\hline & & & & & & & & & & $\begin{array}{l}\text { Mean } \\
\left(\mathrm{ft}^{3} / \mathrm{s}\right)\end{array}$ & $\begin{array}{c}\text { L-scale } \\
\left(\mathrm{ft}^{3} / \mathrm{s}\right)\end{array}$ & L-skew & L-kurtosis \\
\hline 115 & 08065000 & Trinity River near Oakwood, Texas & $31^{\circ} 38^{\prime} 54^{\prime \prime}$ & $95^{\circ} 47^{\prime} 21^{\prime \prime}$ & $\mathrm{NE}$ & & & & 2.55 & 48,348 & $18,871.9$ & 0.336 & 0.164 \\
\hline 116 & 08065200 & Upper Keechi Creek near Oakwood, Texas & $31^{\circ} 34^{\prime} 11^{\prime \prime}$ & $95^{\circ} 53^{\prime} 17^{\prime \prime}$ & SE & 36 & 150 & 5.39 & 8.31 & 6,881 & $4,010.8$ & .433 & .225 \\
\hline 117 & 08065500 & Trinity River near Midway, Texas & $31^{\circ} 04^{\prime} 28^{\prime \prime}$ & $95^{\circ} 41^{\prime} 57^{\prime \prime}$ & SE & 14 & 14,450 & 16.38 & 2.20 & 54,986 & $23,922.0$ & .405 & .149 \\
\hline 118 & 08065700 & Caney Creek near Madisonville, Texas & $30^{\circ} 56^{\prime} 12^{\prime \prime}$ & $95^{\circ} 56^{\prime} 07^{\prime \prime}$ & SE & 13 & 112 & 5.50 & 10.31 & 5,955 & $2,686.7$ & .297 & .020 \\
\hline 119 & 08065800 & Bedias Creek near Madisonville, Texas & $30^{\circ} 53^{\prime} 03^{\prime \prime}$ & $95^{\circ} 46^{\prime} 39^{\prime \prime}$ & SE & 30 & 321 & 4.46 & 7.80 & 12,567 & $5,696.8$ & .280 & .032 \\
\hline 120 & 08066000 & Trinity River at Riverside, Texas & $30^{\circ} 51^{\prime} 33^{\prime \prime}$ & $95^{\circ} 23^{\prime} 55^{\prime \prime}$ & SE & 51 & 15,589 & 18.22 & 2.01 & 43,371 & $14,327.1$ & .225 & .162 \\
\hline 121 & 08066100 & White Rock Creek near Trinity, Texas & $31^{\circ} 03^{\prime} 06^{\prime \prime}$ & $95^{\circ} 22^{\prime} 40^{\prime \prime}$ & SE & 18 & 222 & 4.16 & 9.18 & 7,672 & $3,357.8$ & .178 & .049 \\
\hline 122 & 08066170 & Kickapoo Creek near Onalaska, Texas & $30^{\circ} 54^{\prime} 25^{\prime \prime}$ & $95^{\circ} 05^{\prime} 18^{\prime \prime}$ & SE & 32 & 57 & 2.88 & 12.80 & 8,917 & $4,954.7$ & .662 & .541 \\
\hline 123 & 08066200 & Long King Creek at Livingston, Texas & $30^{\circ} 42^{\prime} 58^{\prime \prime}$ & $94^{\circ} 57^{\prime} 31^{\prime \prime}$ & SE & 35 & 141 & 3.46 & 10.70 & 9,615 & $4,788.3$ & .472 & .287 \\
\hline 124 & 08066300 & Menard Creek near Rye, Texas & $30^{\circ} 28^{\prime} 52^{\prime \prime}$ & $94^{\circ} 46^{\prime} 46^{\prime \prime}$ & SE & 32 & 152 & 10.10 & 7.54 & 3,615 & $1,816.0$ & .431 & .250 \\
\hline 125 & 08066400 & Big Creek near Shepherd, Texas & $30^{\circ} 30^{\prime} 59^{\prime \prime}$ & $94^{\circ} 59^{\prime} 06^{\prime \prime}$ & $\mathrm{SE}$ & 24 & 38.8 & 5.38 & 18.16 & 2,055 & $1,488.9$ & .746 & .640 \\
\hline 126 & 08066500 & Trinity River at Romayor, Texas & $30^{\circ} 25^{\prime} 30^{\prime \prime}$ & $94^{\circ} 51^{\prime} 02^{\prime \prime}$ & SE & 30 & 17,186 & 20.91 & 1.96 & 45,743 & $13,229.3$ & .213 & .178 \\
\hline 127 & 08067000 & Trinity River at Liberty, Texas & $30^{\circ} 03^{\prime} 27^{\prime \prime}$ & $94^{\circ} 49^{\prime} 05^{\prime \prime}$ & SE & 14 & 17,468 & 24.09 & 1.86 & 48,100 & $16,473.6$ & .302 & .167 \\
\hline 128 & 08067500 & Cedar Bayou near Crosby, Texas & $29^{\circ} 58^{\prime} 20^{\prime \prime}$ & $94^{\circ} 59^{\prime} 10^{\prime \prime}$ & SE & 26 & 64.9 & 4.11 & 2.81 & 2,620 & 784.6 & .271 & .197 \\
\hline 129 & 08068000 & West Fork San Jacinto River near Conroe, Texas & $30^{\circ} 14^{\prime} 40^{\prime \prime}$ & $95^{\circ} 27^{\prime} 25^{\prime \prime}$ & SE & 37 & 828 & 3.89 & 5.09 & 17,914 & $9,850.7$ & .457 & .314 \\
\hline 130 & 08068500 & Spring Creek near Spring, Texas & $30^{\circ} 06^{\prime} 37^{\prime \prime}$ & $95^{\circ} 26^{\prime} 10^{\prime \prime}$ & SE & 59 & 409 & 6.40 & 6.15 & 9,110 & $4,856.2$ & .466 & .354 \\
\hline 131 & 08068520 & Spring Creek at Spring, Texas & $30^{\circ} 05^{\prime} 31^{\prime \prime}$ & $95^{\circ} 24^{\prime} 21^{\prime \prime}$ & SE & 58 & 419 & 7.15 & 5.93 & 9,927 & $5,484.6$ & .485 & .355 \\
\hline 132 & 08068720 & Cypress Creek at Katy-Hockley Road near Hockley, Texas & $29^{\circ} 57^{\prime} 00^{\prime \prime}$ & $95^{\circ} 48^{\prime} 29^{\prime \prime}$ & SE & 22 & 110 & 6.05 & 5.85 & 1,246 & 368.5 & .044 & .071 \\
\hline 133 & 08068740 & Cypress Creek at House-Hahl Road near Cypress, Texas & $29^{\circ} 57^{\prime} 32^{\prime \prime}$ & $95^{\circ} 43^{\prime} 03^{\prime \prime}$ & SE & 23 & 131 & 7.68 & 4.96 & 1,610 & 475.7 & .293 & .256 \\
\hline 134 & 08068780 & Little Cypress Creek near Cypress, Texas & $30^{\circ} 00^{\prime} 57^{\prime \prime}$ & $95^{\circ} 41^{\prime} 50^{\prime \prime}$ & SE & 15 & 41 & 4.07 & 5.84 & 1,535 & 652.7 & .333 & .130 \\
\hline 135 & 08068800 & Cypress Creek at Grant Road near Cypress, Texas & $29^{\circ} 58^{\prime} 24^{\prime \prime}$ & $95^{\circ} 35^{\prime} 54^{\prime \prime}$ & SE & 15 & 214 & 8.20 & 5.01 & 3,083 & $1,004.4$ & .300 & .471 \\
\hline 136 & 08069500 & West Fork San Jacinto River near Humble, Texas & $30^{\circ} 01^{\prime} 37^{\prime \prime}$ & $95^{\circ} 15^{\prime} 28^{\prime \prime}$ & $\mathrm{SE}$ & 26 & 1,741 & 3.93 & 4.16 & 33,503 & $19,472.4$ & .613 & .431 \\
\hline 137 & 08070000 & East Fork San Jacinto River near Cleveland, Texas & $30^{\circ} 20^{\prime} 11^{\prime \prime}$ & $95^{\circ} 06^{\prime} 14^{\prime \prime}$ & SE & 58 & 325 & 6.29 & 5.51 & 9,459 & $5,464.9$ & .531 & .354 \\
\hline 138 & 08070200 & East Fork San Jacinto River near New Caney, Texas & $30^{\circ} 08^{\prime} 43^{\prime \prime}$ & $95^{\circ} 07^{\prime} 27^{\prime \prime}$ & SE & 12 & 338 & 11.76 & 4.74 & 10,913 & $7,454.2$ & .800 & .708 \\
\hline 139 & 08070500 & Caney Creek near Splendora, Texas & $30^{\circ} 15^{\prime} 34^{\prime \prime}$ & $95^{\circ} 18^{\prime} 08^{\prime \prime}$ & SE & 54 & 105 & 8.71 & 8.79 & 4,548 & $2,407.0$ & .586 & .447 \\
\hline 140 & 08071000 & Peach Creek at Splendora, Texas & $30^{\circ} 13^{\prime} 57^{\prime \prime}$ & $95^{\circ} 10^{\prime} 05^{\prime \prime}$ & $\mathrm{SE}$ & 34 & 117 & 7.93 & 9.91 & 3,720 & $2,468.4$ & .607 & .428 \\
\hline 141 & 08071280 & Luce Bayou above Lake Houston near Huffman, Texas & $30^{\circ} 06^{\prime} 34^{\prime \prime}$ & $95^{\circ} 03^{\prime} 35^{\prime \prime}$ & SE & 13 & 218 & 8.10 & 6.17 & 7,590 & $4,148.6$ & .468 & .231 \\
\hline 142 & 08071500 & San Jacinto River near Huffman, Texas & $29^{\circ} 59^{\prime} 40^{\prime \prime}$ & $95^{\circ} 08^{\prime} 00^{\prime \prime}$ & SE & 17 & 2,800 & 3.10 & 3.70 & 46,391 & $26,645.7$ & .574 & .392 \\
\hline 143 & 08072300 & Buffalo Bayou near Katy, Texas & $29^{\circ} 44^{\prime} 35^{\prime \prime}$ & $95^{\circ} 48^{\prime} 24^{\prime \prime}$ & SE & 20 & 63.3 & 2.14 & 4.08 & 1,943 & 493.8 & -.001 & 099 \\
\hline
\end{tabular}


Table 1. Streamflow statistics and basin characteristics for 367 USGS streamflow-gaging stations with at least 10 years of annual peak-streamflow data through 1997 (designated as natural by 10-percent criterion)—Continued

\begin{tabular}{|c|c|c|c|c|c|c|c|c|c|c|c|c|c|}
\hline \multirow{2}{*}{$\begin{array}{l}\text { Seq. } \\
\text { no. }\end{array}$} & \multirow{2}{*}{$\begin{array}{c}\text { USGS } \\
\text { station } \\
\text { no. } \\
\text { (pl. 1) }\end{array}$} & \multirow{2}{*}{ USGS station name } & \multirow{2}{*}{ Latitude } & \multirow{2}{*}{ Longitude } & \multirow{2}{*}{ Region } & \multirow{3}{*}{$\begin{array}{c}\begin{array}{c}\text { Years } \\
\text { of } \\
\text { natural } \\
\text { annual } \\
\text { peak } \\
\text { stream- } \\
\text { flow }\end{array} \\
20\end{array}$} & \multirow{3}{*}{$\begin{array}{c}\begin{array}{c}\text { Contri- } \\
\text { buting } \\
\text { drainage } \\
\text { area } \\
\left(\mathrm{mi}^{2}\right)\end{array} \\
21.5\end{array}$} & \multirow{3}{*}{$\begin{array}{r}\begin{array}{c}\text { Basin } \\
\text { shape } \\
\text { factor } \\
\text { (dimen- } \\
\text { sion- } \\
\text { less) }\end{array} \\
4.76\end{array}$} & \multirow{2}{*}{$\begin{array}{c}\text { Stream } \\
\text { slope } \\
\text { (ft/mi) }\end{array}$} & \multicolumn{4}{|c|}{$\begin{array}{l}\text { L-moment statistics of annual } \\
\text { peak-streamflow data }\end{array}$} \\
\hline & & & & & & & & & & $\begin{array}{l}\text { Mean } \\
\left(\mathrm{ft}^{3} / \mathrm{s}\right)\end{array}$ & $\begin{array}{l}\text { L-scale } \\
\left(\mathrm{ft}^{3} / \mathrm{s}\right)\end{array}$ & L-skew & L-kurtosis \\
\hline 144 & 08072730 & Bear Creek near Barker, Texas & $29^{\circ} 49^{\prime} 50^{\prime \prime}$ & $95^{\circ} 41^{\prime} 12^{\prime \prime}$ & SE & & & & 4.96 & 720 & 199.9 & 0.239 & 0.256 \\
\hline 145 & 08072760 & Langham Creek at West Little York Road near Addicks, Texas & $29^{\circ} 52^{\prime} 01^{\prime \prime}$ & $95^{\circ} 38^{\prime} 47^{\prime \prime}$ & $\mathrm{SE}$ & 17 & 24.6 & 3.01 & 4.19 & 1,026 & 293.4 & .041 & .060 \\
\hline 146 & 08072800 & Langham Creek near Addicks, Texas & $29^{\circ} 50^{\prime} 08^{\prime \prime}$ & $95^{\circ} 37^{\prime} 30^{\prime \prime}$ & SE & 11 & 48.9 & 1.86 & 4.81 & 1,432 & 494.1 & .351 & .123 \\
\hline 147 & 08074020 & Whiteoak Bayou at Alabonson Road at Houston, Texas & $29^{\circ} 52^{\prime} 14^{\prime \prime}$ & $95^{\circ} 28^{\prime} 49^{\prime \prime}$ & SE & 13 & 34.5 & 4.49 & 4.22 & 4,340 & $1,341.7$ & .155 & .043 \\
\hline 148 & 08078000 & Chocolate Bayou near Alvin, Texas & $29^{\circ} 22^{\prime} 09^{\prime \prime}$ & $95^{\circ} 19^{\prime} 14^{\prime \prime}$ & SE & 51 & 87.7 & 3.44 & 2.74 & 2,970 & $1,063.5$ & .374 & .407 \\
\hline 149 & 08079500 & $\begin{array}{l}\text { North Fork Double Mountain Fork Brazos River at Lubbock, } \\
\text { Texas }\end{array}$ & $33^{\circ} 35^{\prime} 08^{\prime \prime}$ & $101^{\circ} 49^{\prime} 40^{\prime \prime}$ & $\mathrm{W}$ & 12 & 200 & 80.93 & 7.43 & 464 & 380.8 & .694 & .471 \\
\hline 150 & 08079600 & Double Mountain Fork Brazos River at Justiceburg, Texas & $33^{\circ} 02^{\prime} 18^{\prime \prime}$ & $101^{\circ} 11^{\prime} 50^{\prime \prime}$ & $\mathrm{W}$ & 36 & 244 & 5.61 & 16.67 & 12,207 & $6,224.7$ & .372 & .146 \\
\hline 151 & 08080500 & Double Mountain Fork Brazos River near Aspermont, Texas & $33^{\circ} 00^{\prime} 29^{\prime \prime}$ & $100^{\circ} 10^{\prime} 49^{\prime \prime}$ & $\mathrm{W}$ & 69 & 1,864 & 57.11 & 7.41 & 19,723 & $8,574.1$ & .312 & .202 \\
\hline 152 & 08080540 & McDonald Creek near Post, Texas & $33^{\circ} 21^{\prime} 03^{\prime \prime}$ & $101^{\circ} 13^{\prime} 36^{\prime \prime}$ & $\mathrm{W}$ & 13 & 79.2 & 4.38 & 33.46 & 2,209 & $1,503.2$ & .728 & .645 \\
\hline 153 & 08080700 & Running Water Draw at Plainview, Texas & $34^{\circ} 10^{\prime} 44^{\prime \prime}$ & $101^{\circ} 42^{\prime} 08^{\prime \prime}$ & $\mathrm{W}$ & 37 & 382 & 47.17 & 9.07 & 1,064 & 828.9 & .701 & .526 \\
\hline 154 & 08081200 & Croton Creek near Jayton, Texas & $33^{\circ} 17^{\prime} 18^{\prime \prime}$ & $100^{\circ} 25^{\prime} 52^{\prime \prime}$ & $\mathrm{W}$ & 27 & 290 & 13.49 & 18.99 & 3,686 & $1,543.8$ & .166 & .077 \\
\hline 155 & 08081500 & Salt Croton Creek near Aspermont, Texas & $33^{\circ} 24^{\prime} 03^{\prime \prime}$ & $100^{\circ} 24^{\prime} 29^{\prime \prime}$ & $\mathrm{W}$ & 21 & 64.3 & 3.40 & 23.31 & 5,430 & $3,306.2$ & .541 & .336 \\
\hline 156 & 08082000 & Salt Fork Brazos River near Aspermont, Texas & $33^{\circ} 20^{\prime} 02^{\prime \prime}$ & $100^{\circ} 14^{\prime} 16^{\prime \prime}$ & $\mathrm{W}$ & 27 & 2,496 & 47.49 & 8.62 & 19,570 & $5,858.3$ & .131 & .105 \\
\hline 157 & 08082100 & Stinking Creek near Aspermont, Texas & $33^{\circ} 14^{\prime} 00^{\prime \prime}$ & $100^{\circ} 12^{\prime} 47^{\prime \prime}$ & $\mathrm{W}$ & 18 & 88.8 & 6.96 & 10.69 & 894 & 447.6 & .410 & .187 \\
\hline 158 & 08082180 & North Croton Creek near Knox City, Texas & $33^{\circ} 22^{\prime} 59^{\prime \prime}$ & $100^{\circ} 04^{\prime} 51^{\prime \prime}$ & $\mathrm{W}$ & 21 & 251 & 12.83 & 11.85 & 3,667 & $2,644.1$ & .693 & .555 \\
\hline 159 & 08082500 & Brazos River at Seymour, Texas & $33^{\circ} 34^{\prime} 51^{\prime \prime}$ & $99^{\circ} 16^{\prime} 02^{\prime \prime}$ & $\mathrm{W}$ & 50 & 5,972 & 33.74 & 7.40 & 30,968 & $11,917.5$ & .279 & .124 \\
\hline 160 & 08082700 & Millers Creek near Munday, Texas & $33^{\circ} 19^{\prime} 45^{\prime \prime}$ & $99^{\circ} 27^{\prime} 53^{\prime \prime}$ & $\mathrm{W}$ & 34 & 104 & 8.39 & 7.00 & 1,989 & $1,625.8$ & .779 & .660 \\
\hline 161 & 08083100 & Clear Fork Brazos River near Roby, Texas & $32^{\circ} 47^{\prime} 15^{\prime \prime}$ & $100^{\circ} 23^{\prime} 18^{\prime \prime}$ & $\mathrm{W}$ & 36 & 228 & 6.62 & 13.09 & 1,819 & $1,081.8$ & .434 & .135 \\
\hline 162 & 08083240 & Clear Fork Brazos River at Hawley, Texas & $32^{\circ} 35^{\prime} 53^{\prime \prime}$ & $99^{\circ} 48^{\prime} 53^{\prime \prime}$ & $\mathrm{W}$ & 22 & 1,416 & 8.13 & 7.37 & 2,819 & $1,272.7$ & .447 & .162 \\
\hline 163 & 08083245 & Mulberry Creek near Hawley, Texas & $32^{\circ} 34^{\prime} 04^{\prime \prime}$ & $99^{\circ} 47^{\prime} 32^{\prime \prime}$ & $\mathrm{W}$ & 21 & 205 & 12.06 & 17.69 & 1,178 & 422.7 & .231 & .066 \\
\hline 164 & 08083400 & Little Elm Creek near Abilene, Texas & $32^{\circ} 23^{\prime} 29^{\prime \prime}$ & $99^{\circ} 51^{\prime} 08^{\prime \prime}$ & $\mathrm{W}$ & 16 & 39.1 & 4.72 & 32.85 & 744 & 349.9 & .196 & .031 \\
\hline 165 & 08084000 & Clear Fork Brazos River at Nugent, Texas & $32^{\circ} 41^{\prime} 24^{\prime \prime}$ & $99^{\circ} 40^{\prime} 09^{\prime \prime}$ & $\mathrm{W}$ & 16 & 2,199 & 7.42 & 6.78 & 10,569 & $4,782.8$ & .509 & .426 \\
\hline 166 & 08084800 & California Creek near Stamford, Texas & $32^{\circ} 55^{\prime} 51^{\prime \prime}$ & $99^{\circ} 38^{\prime} 32^{\prime \prime}$ & $\mathrm{W}$ & 35 & 478 & 8.81 & 7.58 & 3,564 & $2,078.3$ & .602 & .532 \\
\hline 167 & 08085500 & Clear Fork Brazos River at Fort Griffin, Texas & $32^{\circ} 56^{\prime} 04^{\prime \prime}$ & $99^{\circ} 13^{\prime} 27^{\prime \prime}$ & $\mathrm{w}$ & 16 & 3988 & 12.39 & 5.28 & 11,462 & $4,540.7$ & .360 & .190 \\
\hline 168 & 08086050 & Deep Creek at Moran, Texas & $32^{\circ} 33^{\prime} 33^{\prime \prime}$ & $99^{\circ} 10^{\prime} 11^{\prime \prime}$ & $\mathrm{W}$ & 13 & 228 & 6.42 & 16.47 & 5,119 & $1,520.3$ & -.165 & .188 \\
\hline 169 & 08086100 & Hubbard Creek near Albany, Texas & $32^{\circ} 41^{\prime} 21^{\prime \prime}$ & $99^{\circ} 09^{\prime} 52^{\prime \prime}$ & $\mathrm{W}$ & 14 & 454 & 5.68 & 13.76 & 8,204 & $3,099.7$ & .045 & -.051 \\
\hline 170 & 08086150 & North Fork Hubbard Creek near Albany, Texas & $32^{\circ} 42^{\prime} 27^{\prime \prime}$ & $99^{\circ} 16^{\prime} 29^{\prime \prime}$ & $\mathrm{W}$ & 28 & 39.3 & 4.57 & 36.26 & 6,071 & $5,084.6$ & .825 & .732 \\
\hline 171 & 08086212 & Hubbard Creek below Albany, Texas & $32^{\circ} 43^{\prime} 58^{\prime \prime}$ & $99^{\circ} 08^{\prime} 25^{\prime \prime}$ & $\mathrm{W}$ & 31 & 613 & 5.18 & 12.75 & 20,784 & $15,490.6$ & .718 & .620 \\
\hline
\end{tabular}


$\omega \quad$ Table 1. Streamflow statistics and basin characteristics for 367 USGS streamflow-gaging stations with at least 10 years of annual peak-streamflow data through 1997 (designated as natural by 10-percent criterion)-Continued

\begin{tabular}{|c|c|c|c|c|c|c|c|c|c|c|c|c|c|}
\hline \multirow{2}{*}{$\begin{array}{l}\text { Seq. } \\
\text { no. }\end{array}$} & \multirow{2}{*}{$\begin{array}{c}\text { USGS } \\
\text { station } \\
\text { no. } \\
\text { (pl. 1) }\end{array}$} & \multirow{2}{*}{ USGS station name } & \multirow{2}{*}{ Latitude } & \multirow{2}{*}{ Longitude } & \multirow{2}{*}{ Region } & \multirow{3}{*}{$\begin{array}{c}\begin{array}{c}\text { Years } \\
\text { of } \\
\text { natural } \\
\text { annual } \\
\text { peak } \\
\text { stream- } \\
\text { flow }\end{array} \\
22\end{array}$} & \multirow{3}{*}{$\begin{array}{c}\begin{array}{c}\text { Contri- } \\
\text { buting } \\
\text { drainage } \\
\text { area } \\
\left(\mathbf{m i}^{2}\right)\end{array} \\
280\end{array}$} & \multirow{3}{*}{$\begin{array}{c}\begin{array}{c}\text { Basin } \\
\text { shape } \\
\text { factor } \\
\text { (dimen- } \\
\text { sion- } \\
\text { less) }\end{array} \\
28.10\end{array}$} & \multirow{2}{*}{$\begin{array}{c}\text { Stream } \\
\text { slope } \\
(\mathrm{ft} / \mathrm{mi})\end{array}$} & \multicolumn{4}{|c|}{$\begin{array}{l}\text { L-moment statistics of annual } \\
\text { peak-streamflow data }\end{array}$} \\
\hline & & & & & & & & & & $\begin{array}{l}\text { Mean } \\
\left(\mathrm{ft}^{3} / \mathrm{s}\right)\end{array}$ & $\begin{array}{l}\text { L-scale } \\
\left(\mathrm{ft}^{3} / \mathrm{s}\right)\end{array}$ & L-skew & L-kurtosis \\
\hline 172 & 08086290 & Big Sandy Creek above Breckenridge, Texas & $32^{\circ} 38^{\prime} 54^{\prime \prime}$ & $99^{\circ} 00^{\prime} 15^{\prime \prime}$ & $\mathrm{W}$ & & & & 8.10 & 7,686 & $5,043.5$ & 0.744 & 0.683 \\
\hline 173 & 08086300 & Big Sandy Creek near Breckenridge, Texas & $32^{\circ} 39^{\prime} 52^{\prime \prime}$ & $99^{\circ} 00^{\prime} 01^{\prime \prime}$ & $\mathrm{W}$ & 14 & 298 & 23.90 & 8.63 & 5,086 & $1,200.9$ & .084 & -.017 \\
\hline 174 & 08087300 & Clear Fork Brazos River at Eliasville, Texas & $32^{\circ} 57^{\prime} 36^{\prime \prime}$ & $98^{\circ} 45^{\prime} 59^{\prime \prime}$ & $\mathrm{W}$ & 31 & 5697 & 14.22 & 4.81 & 12,597 & $3,955.4$ & .192 & .147 \\
\hline 175 & 08088000 & Brazos River near South Bend, Texas & $33^{\circ} 01^{\prime} 27^{\prime \prime}$ & $98^{\circ} 38^{\prime} 37^{\prime \prime}$ & $\mathrm{W}$ & 23 & 13,107 & 22.41 & 6.47 & 30,575 & $10,669.8$ & .237 & .178 \\
\hline 176 & 08088100 & Salt Creek at Olney, Texas & $33^{\circ} 22^{\prime} 13^{\prime \prime}$ & $98^{\circ} 44^{\prime} 40^{\prime \prime}$ & $\mathrm{W}$ & 20 & 11.8 & 1.71 & 13.13 & 913 & 550.2 & .645 & .419 \\
\hline 177 & 08088300 & Briar Creek near Graham, Texas & $33^{\circ} 12^{\prime} 43^{\prime \prime}$ & $98^{\circ} 37^{\prime} 06^{\prime \prime}$ & $\mathrm{W}$ & 31 & 24.2 & 6.09 & 15.41 & 1,114 & 573.7 & .387 & .137 \\
\hline 178 & 08088450 & Big Cedar Creek near Ivan, Texas & $32^{\circ} 49^{\prime} 39^{\prime \prime}$ & $98^{\circ} 43^{\prime} 25^{\prime \prime}$ & $\mathrm{W}$ & 25 & 97 & 7.00 & 13.47 & 4,247 & $2,769.3$ & .525 & .367 \\
\hline 179 & 08089000 & Brazos River near Palo Pinto, Texas & $32^{\circ} 51^{\prime} 45^{\prime \prime}$ & $98^{\circ} 18^{\prime} 08^{\prime \prime}$ & $\mathrm{NE}$ & 17 & 14,245 & 27.66 & 5.93 & 41,201 & $11,145.9$ & .176 & .262 \\
\hline 180 & 08090500 & Palo Pinto Creek near Santo, Texas & $32^{\circ} 37^{\prime} 51^{\prime \prime}$ & $98^{\circ} 10^{\prime} 50^{\prime \prime}$ & $\mathrm{NE}$ & 13 & 573 & 4.92 & 10.20 & 15,132 & $6,750.4$ & .357 & .220 \\
\hline 181 & 08091000 & Brazos River near Glen Rose, Texas & $32^{\circ} 16^{\prime} 18^{\prime \prime}$ & $97^{\circ} 39^{\prime} 48^{\prime \prime}$ & $\mathrm{NE}$ & 17 & 16,252 & 37.82 & 5.04 & 41,961 & $11,231.5$ & .186 & .341 \\
\hline 182 & 08091500 & Paluxy River at Glen Rose, Texas & $32^{\circ} 13^{\prime} 53^{\prime \prime}$ & $97^{\circ} 46^{\prime} 37^{\prime \prime}$ & $\mathrm{NE}$ & 35 & 410 & 8.13 & 13.64 & 15,356 & $7,548.9$ & .353 & .110 \\
\hline 183 & 08092000 & Nolan River at Blum, Texas & $32^{\circ} 09^{\prime} 02^{\prime \prime}$ & $97^{\circ} 24^{\prime} 09^{\prime \prime}$ & $\mathrm{NE}$ & 16 & 282 & 3.81 & 13.11 & 12,354 & $3,365.8$ & .195 & .267 \\
\hline 184 & 08093250 & Hackberry Creek at Hillsboro, Texas & $32^{\circ} 00^{\prime} 20^{\prime \prime}$ & $97^{\circ} 08^{\prime} 59^{\prime \prime}$ & NE & 13 & 57.9 & 3.73 & 9.15 & 4,995 & $2,198.9$ & .248 & -.036 \\
\hline 185 & 08093500 & Aquilla Creek near Aquilla, Texas & $31^{\circ} 50^{\prime} 40^{\prime \prime}$ & $97^{\circ} 12^{\prime} 04^{\prime \prime}$ & $\mathrm{NE}$ & 44 & 308 & 4.90 & 8.19 & 12,840 & $5,215.6$ & .369 & .250 \\
\hline 186 & 08094000 & Green Creek Subwatershed No. 1 near McKinney, Texas & $32^{\circ} 09^{\prime} 57^{\prime \prime}$ & $98^{\circ} 20^{\prime} 28^{\prime \prime}$ & $\mathrm{NE}$ & 22 & 4.19 & 1.83 & 41.45 & 1,624 & $1,002.6$ & .610 & .435 \\
\hline 187 & 08095000 & North Bosque River near Clifton, Texas & $31^{\circ} 47^{\prime} 09^{\prime \prime}$ & $97^{\circ} 34^{\prime} 04^{\prime \prime}$ & $\mathrm{NE}$ & 44 & 968 & 9.28 & 9.69 & 22,369 & $7,996.3$ & .176 & .137 \\
\hline 188 & 08095300 & Middle Bosque River near McGregor, Texas & $31^{\circ} 30^{\prime} 33^{\prime \prime}$ & $97^{\circ} 21^{\prime} 56^{\prime \prime}$ & $\mathrm{NE}$ & 38 & 182 & 5.44 & 19.39 & 13,633 & $5,214.8$ & .189 & .112 \\
\hline 189 & 08095400 & Hog Creek near Crawford, Texas & $31^{\circ} 33^{\prime} 20^{\prime \prime}$ & $97^{\circ} 21^{\prime 22 "}$ & $\mathrm{NE}$ & 20 & 78.2 & 15.25 & 17.20 & 7,451 & $2,330.4$ & .043 & .128 \\
\hline 190 & 08096500 & Brazos River at Waco, Texas & $31^{\circ} 32^{\prime} 06^{\prime \prime}$ & $97^{\circ} 04^{\prime} 22^{\prime \prime}$ & $\mathrm{NE}$ & 43 & 20,007 & 39.89 & 4.68 & 65,053 & $26,023.4$ & .335 & .166 \\
\hline 191 & 08096800 & Cow Bayou Subwatershed No. 4 near Bruceville, Texas & $31^{\circ} 19^{\prime} 59^{\prime \prime}$ & $97^{\circ} 16^{\prime} 02^{\prime \prime}$ & $\mathrm{NE}$ & 18 & 5.04 & 3.36 & 43.90 & 1,004 & 414.2 & .050 & -.020 \\
\hline 192 & 08098203 & Brushy Creek Watershed C near Riessel, Texas & $31^{\circ} 31^{\prime} 11^{\prime \prime}$ & $96^{\circ} 53^{\prime} 34^{\prime \prime}$ & $\mathrm{NE}$ & 32 & .90 & 1.60 & 32.83 & 320 & 128.1 & .252 & .114 \\
\hline 193 & 08098206 & Brushy Creek Watershed D near Riessel, Texas & $31^{\circ} 30^{\prime} 38^{\prime \prime}$ & $96^{\circ} 53^{\prime} 32^{\prime \prime}$ & $\mathrm{NE}$ & 15 & 1.73 & 1.97 & 28.44 & 514 & 188.1 & .080 & -.017 \\
\hline 194 & 08098227 & Brushy Creek Watershed Y-2 near Riessel, Texas & $31^{\circ} 28^{\prime} 30^{\prime \prime}$ & $96^{\circ} 52^{\prime} 46^{\prime \prime}$ & $\mathrm{NE}$ & 37 & .21 & .69 & 60.44 & 137 & 70.5 & .307 & .108 \\
\hline 195 & 08098239 & Brushy Creek Watershed Y near Riessel, Texas & $31^{\circ} 28^{\prime} 36^{\prime \prime}$ & $96^{\circ} 52^{\prime} 36^{\prime \prime}$ & $\mathrm{NE}$ & 36 & .48 & .88 & 50.47 & 260 & 134.7 & .280 & .044 \\
\hline 196 & 08098242 & Brushy Creek Watershed G near Riessel, Texas & $31^{\circ} 288^{\prime} 59^{\prime \prime}$ & $96^{\circ} 52^{\prime} 06^{\prime \prime}$ & $\mathrm{NE}$ & 24 & 6.84 & 4.10 & 17.97 & 1,189 & 539.7 & .241 & .116 \\
\hline 197 & 08098263 & Brushy Creek Watershed W-1 near Riessel, Texas & $31^{\circ} 27^{\prime} 27^{\prime \prime}$ & $96^{\circ} 52^{\prime} 48^{\prime \prime}$ & $\mathrm{NE}$ & 38 & .28 & 1.10 & 65.04 & 236 & 106.7 & .188 & .050 \\
\hline 198 & 08098281 & Brushy Creek Watershed W-2 near Riessel, Texas & $31^{\circ} 27^{\prime} 19^{\prime \prime}$ & $96^{\circ} 52^{\prime} 55^{\prime \prime}$ & $\mathrm{NE}$ & 38 & .20 & 3.44 & 56.63 & 149 & 77.2 & .299 & .103 \\
\hline 199 & 08098300 & Little Pond Creek near Burlington, Texas & $31^{\circ} 01^{\prime} 35^{\prime \prime}$ & $96^{\circ} 59^{\prime} 17^{\prime \prime}$ & SE & 20 & 23 & 8.36 & 12.06 & 3,373 & $1,336.0$ & .141 & .097 \\
\hline 200 & 08099300 & Sabana River near De Leon, Texas & $32^{\circ} 06^{\prime} 50^{\prime \prime}$ & $98^{\circ} 36^{\prime} 19^{\prime \prime}$ & $\mathrm{W}$ & 19 & 264 & 11.63 & 12.37 & 5,332 & $1,858.3$ & .057 & -.002 \\
\hline
\end{tabular}


Table 1. Streamflow statistics and basin characteristics for 367 USGS streamflow-gaging stations with at least 10 years of annual peak-streamflow data through 1997 (designated as natural by 10-percent criterion)—Continued

\begin{tabular}{|c|c|c|c|c|c|c|c|c|c|c|c|c|c|}
\hline \multirow{2}{*}{$\begin{array}{l}\text { Seq. } \\
\text { no. }\end{array}$} & \multirow{2}{*}{$\begin{array}{l}\text { USGS } \\
\text { station } \\
\text { no. } \\
\text { (pl. 1) }\end{array}$} & \multirow{2}{*}{ USGS station name } & \multirow{2}{*}{ Latitude } & \multirow{2}{*}{ Longitude } & \multirow{2}{*}{ Region } & \multirow{2}{*}{$\begin{array}{c}\text { Years } \\
\text { of } \\
\text { natural } \\
\text { annual } \\
\text { peak } \\
\text { stream- } \\
\text { flow }\end{array}$} & \multirow{2}{*}{$\begin{array}{l}\text { Contri- } \\
\text { buting } \\
\text { drainage } \\
\text { area } \\
\left(\mathrm{mi}^{2}\right)\end{array}$} & \multirow{2}{*}{$\begin{array}{c}\text { Basin } \\
\text { shape } \\
\text { factor } \\
\text { (dimen- } \\
\text { sion- } \\
\text { less) }\end{array}$} & \multirow{2}{*}{$\begin{array}{l}\text { Stream } \\
\text { slope } \\
\text { (ft/mi) }\end{array}$} & \multicolumn{4}{|c|}{$\begin{array}{l}\text { L-moment statistics of annual } \\
\text { peak-streamflow data }\end{array}$} \\
\hline & & & & & & & & & & $\begin{array}{l}\text { Mean } \\
\left(\mathrm{ft}^{3} / \mathrm{s}\right)\end{array}$ & $\begin{array}{l}\text { L-scale } \\
\left(\mathrm{ft}^{3} / \mathrm{s}\right)\end{array}$ & L-skew & L-kurtosis \\
\hline 201 & 08099500 & Leon River near Hasse, Texas & $31^{\circ} 57^{\prime} 28^{\prime \prime}$ & $98^{\circ} 27^{\prime} 32^{\prime \prime}$ & $\mathrm{W}$ & 15 & 1,261 & 6.79 & 9.33 & 10,781 & $5,295.6$ & 0.256 & 0.086 \\
\hline 202 & 08100500 & Leon River at Gatesville, Texas & $31^{\circ} 25^{\prime} 58^{\prime \prime}$ & $97^{\circ} 45^{\prime} 42^{\prime \prime}$ & $\mathrm{NE}$ & 13 & 2,342 & 19.69 & 5.81 & 14,398 & $8,130.1$ & .443 & .160 \\
\hline 203 & 08101000 & Cowhouse Creek at Pidcoke, Texas & $31^{\circ} 17^{\prime} 05^{\prime \prime}$ & $97^{\circ} 53^{\prime} 05^{\prime \prime}$ & $\mathrm{NE}$ & 46 & 455 & 11.36 & 13.24 & 17,507 & $8,539.3$ & .334 & .178 \\
\hline 204 & 08102500 & Leon River near Belton, Texas & $31^{\circ} 04^{\prime} 12^{\prime \prime}$ & $97^{\circ} 26^{\prime} 28^{\prime \prime}$ & $\mathrm{NE}$ & 31 & 3,542 & 24.76 & 5.09 & 19,754 & $6,704.5$ & .261 & .158 \\
\hline 205 & 08103800 & Lampasas River near Kempner, Texas & $31^{\circ} 04^{\prime} 54^{\prime \prime}$ & $98^{\circ} 00^{\prime} 59^{\prime \prime}$ & $\mathrm{W}$ & 11 & 818 & 5.22 & 13.46 & 19,529 & $8,634.9$ & .414 & .496 \\
\hline 206 & 08103900 & South Fork Rocky Creek near Briggs, Texas & $30^{\circ} 54^{\prime} 41^{\prime \prime}$ & $98^{\circ} 02^{\prime} 12^{\prime \prime}$ & $\mathrm{W}$ & 35 & 33.3 & 3.93 & 36.15 & 4,612 & $2,471.4$ & .419 & .299 \\
\hline 207 & 08104000 & Lampasas River at Youngsport, Texas & $30^{\circ} 57^{\prime} 26^{\prime \prime}$ & $97^{\circ} 42^{\prime} 30^{\prime \prime}$ & $\mathrm{NE}$ & 49 & 1,240 & 8.33 & 10.49 & 21,614 & $9,314.4$ & .370 & .278 \\
\hline 208 & 08104700 & North Fork San Gabriel River near Georgetown, Texas & $30^{\circ} 39^{\prime} 42^{\prime \prime}$ & $97^{\circ} 42^{\prime} 40^{\prime \prime}$ & $\mathrm{W}$ & 11 & 248 & 9.17 & 16.30 & 10,615 & $5,920.7$ & .442 & .268 \\
\hline 209 & 08104900 & South Fork San Gabriel River at Georgetown, Texas & $30^{\circ} 37^{\prime} 32^{\prime \prime}$ & $97^{\circ} 41^{\prime 2} 27^{\prime \prime}$ & $\mathrm{W}$ & 30 & 133 & 10.88 & 19.84 & 9,793 & $4,164.9$ & .277 & .152 \\
\hline 210 & 08105000 & San Gabriel River at Georgetown, Texas & $30^{\circ} 39^{\prime} 14^{\prime \prime}$ & $97^{\circ} 39^{\prime} 18^{\prime \prime}$ & $\mathrm{W}$ & 39 & 405 & 6.73 & 16.02 & 19,983 & $10,164.5$ & .452 & .374 \\
\hline 211 & 08105100 & Berry Creek near Georgetown, Texas & $30^{\circ} 41^{\prime} 28^{\prime \prime}$ & $97^{\circ} 39^{\prime} 21^{\prime \prime}$ & $\mathrm{W}$ & 30 & 83.1 & 9.44 & 16.04 & 5,177 & $2,234.2$ & .203 & .120 \\
\hline 212 & 08105400 & San Gabriel River near Circleville, Texas & $30^{\circ} 37^{\prime} 43^{\prime \prime}$ & $97^{\circ} 28^{\prime} 23^{\prime \prime}$ & $\mathrm{W}$ & 19 & 599 & 7.29 & 14.60 & 22,140 & $9,344.9$ & .223 & .000 \\
\hline 213 & 08105700 & San Gabriel River at Laneport, Texas & $30^{\circ} 41^{\prime} 39^{\prime \prime}$ & $97^{\circ} 16^{\prime} 43^{\prime \prime}$ & $\mathrm{W}$ & 15 & 738 & 9.59 & 12.68 & 15,342 & $5,369.5$ & .074 & .062 \\
\hline 214 & 08106500 & Little River at Cameron, Texas & $30^{\circ} 49^{\prime} 53^{\prime \prime}$ & $96^{\circ} 57^{\prime} 01 "$ & $\mathrm{SE}$ & 36 & 7065 & 20.21 & 4.46 & 56,703 & $34,120.7$ & .603 & .458 \\
\hline 215 & 08108200 & North Elm Creek near Cameron, Texas & $30^{\circ} 55^{\prime} 52^{\prime \prime}$ & $97^{\circ} 01^{\prime} 13^{\prime \prime}$ & SE & 11 & 44.8 & 10.88 & 10.25 & 3,418 & $1,237.5$ & -.025 & .112 \\
\hline 216 & 08109000 & Brazos River near Bryan, Texas & $30^{\circ} 36^{\prime} 52^{\prime \prime}$ & $96^{\circ} 29^{\prime} 10^{\prime \prime}$ & $\mathrm{SE}$ & 20 & 29,949 & 34.50 & 4.30 & 77,530 & $18,775.3$ & .230 & .104 \\
\hline 217 & 08109700 & Middle Yegua Creek near Dime Box, Texas & $30^{\circ} 20^{\prime} 21^{\prime \prime}$ & $96^{\circ} 54^{\prime} 16^{\prime \prime}$ & SE & 35 & 236 & 7.15 & 6.79 & 2,736 & $1,555.0$ & .351 & .160 \\
\hline 218 & 08109800 & East Yegua Creek near Dime Box, Texas & $30^{\circ} 24^{\prime} 26^{\prime \prime}$ & $96^{\circ} 49^{\prime} 02^{\prime \prime}$ & $\mathrm{SE}$ & 35 & 244 & 4.97 & 7.25 & 3,065 & $1,634.1$ & .350 & .196 \\
\hline 219 & 08110000 & Yegua Creek near Somerville, Texas & $30^{\circ} 19^{\prime} 18^{\prime \prime}$ & $96^{\circ} 30^{\prime} 26^{\prime \prime}$ & $\mathrm{SE}$ & 42 & 1,009 & 3.79 & 4.14 & 11,064 & $5,842.9$ & .384 & .226 \\
\hline 220 & 08110100 & Davidson Creek near Lyons, Texas & $30^{\circ} 25^{\prime} 10^{\prime \prime}$ & $96^{\circ} 32 ' 24^{\prime \prime}$ & $\mathrm{SE}$ & 35 & 195 & 8.01 & 8.30 & 5,842 & $3,009.4$ & .377 & .211 \\
\hline 221 & 08110430 & Big Creek near Freestone, Texas & $31^{\circ} 30^{\prime} 25^{\prime \prime}$ & $96^{\circ} 19^{\prime} 31 "$ & $\mathrm{NE}$ & 19 & 57.1 & 6.32 & 7.25 & 3,051 & $1,642.1$ & .545 & .429 \\
\hline 222 & 08110500 & Navasota River near Easterly, Texas & $31^{\circ} 10^{\prime} 12^{\prime \prime}$ & $96^{\circ} 17^{\prime} 51^{\prime \prime}$ & SE & 37 & 968 & 7.58 & 3.83 & 17,774 & $8,421.2$ & .292 & .131 \\
\hline 223 & 08111000 & Navasota River near Bryan, Texas & $30^{\circ} 52^{\prime} 10^{\prime \prime}$ & $96^{\circ} 11^{\prime} 32^{\prime \prime}$ & SE & 11 & 1,454 & 10.12 & 3.30 & 14,655 & $7,355.6$ & .244 & -.069 \\
\hline 224 & 08111700 & Mill Creek near Bellville, Texas & $29^{\circ} 52^{\prime} 51^{\prime \prime}$ & $96^{\circ} 12^{\prime} 18^{\prime \prime}$ & SE & 30 & 376 & 5.30 & 8.01 & 15,413 & $6,257.8$ & .215 & .137 \\
\hline 225 & 08114000 & Brazos River at Richmond, Texas & $29^{\circ} 34^{\prime} 56^{\prime \prime}$ & $95^{\circ} 45^{\prime} 27^{\prime \prime}$ & $\mathrm{SE}$ & 21 & 35,441 & 41.16 & 3.73 & 62,867 & $13,301.0$ & .065 & .122 \\
\hline 226 & 08115000 & Big Creek near Needville, Texas & $29^{\circ} 28^{\prime} 35^{\prime \prime}$ & $95^{\circ} 48^{\prime} 45^{\prime \prime}$ & $\mathrm{SE}$ & 50 & 42.8 & 4.89 & 2.83 & 2,972 & 966.8 & .295 & .226 \\
\hline 227 & 08116400 & Dry Creek near Rosenberg, Texas & $29^{\circ} 30^{\prime} 42^{\prime \prime}$ & $95^{\circ} 44^{\prime} 48^{\prime \prime}$ & $\mathrm{SE}$ & 21 & 8.65 & 3.93 & 3.38 & 816 & 222.7 & .353 & .364 \\
\hline 228 & 08117500 & San Bernard River near Boling, Texas & $29^{\circ} 18^{\prime} 48^{\prime \prime}$ & $95^{\circ} 53^{\prime} 37^{\prime \prime}$ & SE & 43 & 727 & 9.58 & 4.01 & 8,629 & $3,091.1$ & .248 & .071 \\
\hline 229 & 08119000 & Bluff Creek near Ira, Texas & $32^{\circ} 35^{\prime} 29^{\prime \prime}$ & $101^{\circ} 03^{\prime} 02^{\prime \prime}$ & $\mathrm{W}$ & 18 & 42.6 & 8.56 & 18.72 & 1,204 & 480.2 & .371 & .441 \\
\hline
\end{tabular}


ผ Table 1. Streamflow statistics and basin characteristics for 367 USGS streamflow-gaging stations with at least 10 years of annual peak-streamflow data through 1997 (designated as natural by 10-percent criterion)-Continued

\begin{tabular}{|c|c|c|c|c|c|c|c|c|c|c|c|c|c|}
\hline \multirow{2}{*}{$\begin{array}{l}\text { Seq. } \\
\text { no. }\end{array}$} & \multirow{2}{*}{$\begin{array}{c}\text { USGS } \\
\text { station } \\
\text { no. } \\
\text { (pl. 1) }\end{array}$} & \multirow{2}{*}{ USGS station name } & \multirow{2}{*}{ Latitude } & \multirow{2}{*}{ Longitude } & \multirow{2}{*}{ Region } & \multirow{2}{*}{$\begin{array}{c}\text { Years } \\
\text { of } \\
\text { natural } \\
\text { annual } \\
\text { peak } \\
\text { stream- } \\
\text { flow }\end{array}$} & \multirow{2}{*}{$\begin{array}{l}\text { Contri- } \\
\text { buting } \\
\text { drainage } \\
\text { area } \\
\left(\mathrm{mi}^{2}\right)\end{array}$} & \multirow{2}{*}{$\begin{array}{c}\text { Basin } \\
\text { shape } \\
\text { factor } \\
\text { (dimen- } \\
\text { sion- } \\
\text { less) }\end{array}$} & \multirow{2}{*}{$\begin{array}{c}\text { Stream } \\
\text { slope } \\
(\mathrm{ft} / \mathrm{mi})\end{array}$} & \multicolumn{4}{|c|}{$\begin{array}{l}\text { L-moment statistics of annual } \\
\text { peak-streamflow data }\end{array}$} \\
\hline & & & & & & & & & & $\begin{array}{l}\text { Mean } \\
\left(\mathrm{ft}^{3} / \mathrm{s}\right)\end{array}$ & $\begin{array}{c}\text { L-scale } \\
\left(\mathrm{ft}^{3} / \mathrm{s}\right)\end{array}$ & L-skew & L-kurtosis \\
\hline 230 & 08120500 & Deep Creek near Dunn, Texas & $32^{\circ} 34^{\prime} 25^{\prime \prime}$ & $100^{\circ} 54^{\prime} 27^{\prime \prime}$ & $\mathrm{W}$ & 34 & 188 & 10.85 & 14.02 & 3,651 & $1,919.1$ & 0.504 & 0.408 \\
\hline 231 & 08123500 & Champion Creek near Colorado City, Texas & $32^{\circ} 19^{\prime} 01^{\prime \prime}$ & $100^{\circ} 49^{\prime} 28^{\prime \prime}$ & $\mathrm{W}$ & 12 & 177 & 4.58 & 16.82 & 4,633 & $2,020.8$ & .124 & -.150 \\
\hline 232 & 08124000 & Colorado River at Robert Lee, Texas & $31^{\circ} 53^{\prime} 07^{\prime \prime}$ & $100^{\circ} 28^{\prime} 49^{\prime \prime}$ & $\mathrm{W}$ & 14 & 5047 & 4.04 & 4.64 & 20,764 & $4,666.0$ & -.081 & .078 \\
\hline 233 & 08126500 & Colorado River at Ballinger, Texas & $31^{\circ} 43^{\prime} 58^{\prime \prime}$ & $99^{\circ} 57^{\prime} 13^{\prime \prime}$ & $\mathrm{W}$ & 61 & 6160 & 6.23 & 4.30 & 19,320 & $6,507.0$ & .245 & .200 \\
\hline 234 & 08127000 & Elm Creek at Ballinger, Texas & $31^{\circ} 44^{\prime} 57^{\prime \prime}$ & $99^{\circ} 56^{\prime} 51^{\prime \prime}$ & $\mathrm{W}$ & 49 & 450 & 4.29 & 16.49 & 9,533 & $4,869.8$ & .471 & .290 \\
\hline 235 & 08128000 & South Concho River at Christoval, Texas & $31^{\circ} 11^{\prime} 15^{\prime \prime}$ & $100^{\circ} 30^{\prime} 06^{\prime \prime}$ & $\mathrm{w}$ & 67 & 354 & 3.24 & 13.37 & 9,654 & $7,261.9$ & .641 & .434 \\
\hline 236 & 08128400 & Middle Concho River above Tankersley, Texas & $31^{\circ} 25^{\prime} 38^{\prime \prime}$ & $100^{\circ} 42^{\prime} 39^{\prime \prime}$ & $\mathrm{W}$ & 37 & 1,611 & 6.47 & 7.61 & 3,885 & $2,264.4$ & .323 & .065 \\
\hline 237 & 08128500 & Middle Concho River near Tankersley, Texas & $31^{\circ} 22^{\prime} 35^{\prime \prime}$ & $100^{\circ} 36^{\prime} 50^{\prime \prime}$ & $\mathrm{W}$ & 31 & 1,685 & 7.39 & 7.56 & 11,286 & $4,795.0$ & .174 & .019 \\
\hline 238 & 08129300 & Spring Creek above Tankersley, Texas & $31^{\circ} 19^{\prime} 48^{\prime \prime}$ & $100^{\circ} 38^{\prime} 24^{\prime \prime}$ & $\mathrm{W}$ & 37 & 405 & 7.33 & 13.36 & 2,962 & $2,384.7$ & .673 & .405 \\
\hline 239 & 08130500 & Dove Creek at Knickerbocker, Texas & $31^{\circ} 16^{\prime} 24^{\prime \prime}$ & $100^{\circ} 37^{\prime} 45^{\prime \prime}$ & $\mathrm{W}$ & 37 & 198 & 5.74 & 15.66 & 3,883 & $2,586.7$ & .432 & .111 \\
\hline 240 & 08131000 & Spring Creek near Tankersley, Texas & $31^{\circ} 21^{\prime} 30^{\prime \prime}$ & $100^{\circ} 32^{\prime} 05^{\prime \prime}$ & $\mathrm{W}$ & 30 & 671 & 5.73 & 13.17 & 12,925 & $6,812.4$ & .398 & .301 \\
\hline 241 & 08131400 & Pecan Creek near San Angelo, Texas & $31^{\circ} 18^{\prime} 32^{\prime \prime}$ & $100^{\circ} 26^{\prime} 44^{\prime \prime}$ & $\mathrm{W}$ & 25 & 81.1 & 4.82 & 23.24 & 1,907 & $1,602.2$ & .774 & .628 \\
\hline 242 & 08133500 & North Concho River at Sterling City, Texas & $31^{\circ} 49^{\prime} 48^{\prime \prime}$ & $100^{\circ} 59^{\prime} 36^{\prime \prime}$ & $\mathrm{W}$ & 57 & 568 & 4.10 & 10.60 & 2,940 & $1,921.7$ & .497 & .249 \\
\hline 243 & 08134000 & North Concho River near Carlsbad, Texas & $31^{\circ} 35^{\prime} 33^{\prime \prime}$ & $100^{\circ} 38^{\prime} 12^{\prime \prime}$ & $\mathrm{w}$ & 73 & 1,191 & 5.90 & 9.44 & 11,962 & $8,271.4$ & .572 & .315 \\
\hline 244 & 08135000 & North Concho River at San Angelo, Texas & $31^{\circ} 27^{\prime} 57^{\prime \prime}$ & $100^{\circ} 26^{\prime} 51^{\prime \prime}$ & $\mathrm{W}$ & 17 & 1,450 & 7.74 & 8.30 & 14,104 & $8,245.9$ & .389 & .077 \\
\hline 245 & 08136000 & Concho River at San Angelo, Texas & $31^{\circ} 27^{\prime} 16^{\prime \prime}$ & $100^{\circ} 24^{\prime} 37^{\prime \prime}$ & $\mathrm{W}$ & 15 & 4,411 & 3.87 & 7.53 & 25,635 & $12,303.7$ & .349 & .182 \\
\hline 246 & 08136500 & Concho River at Paint Rock, Texas & $31^{\circ} 30^{\prime} 57^{\prime \prime}$ & $99^{\circ} 55^{\prime} 09^{\prime \prime}$ & $\mathrm{W}$ & 15 & 5,443 & 5.46 & 6.87 & 21,979 & $10,227.5$ & .361 & .179 \\
\hline 247 & 08138000 & Colorado River at Winchell, Texas & $31^{\circ} 28^{\prime} 04^{\prime \prime}$ & $99^{\circ} 09^{\prime} 43^{\prime \prime}$ & $\mathrm{W}$ & 24 & 13,788 & 6.41 & 3.97 & 30,194 & $7,388.4$ & .158 & .259 \\
\hline 248 & 08144500 & San Saba River at Menard, Texas & $30^{\circ} 55^{\prime} 08^{\prime \prime}$ & $99^{\circ} 47^{\prime} 07^{\prime \prime}$ & $\mathrm{w}$ & 78 & 1,128 & 3.06 & 9.55 & 16,187 & $11,078.6$ & .536 & .276 \\
\hline 249 & 08144600 & San Saba River near Brady, Texas & $31^{\circ} 00^{\prime} 14^{\prime \prime}$ & $99^{\circ} 16^{\prime} 07^{\prime \prime}$ & $\mathrm{W}$ & 15 & 1,626 & 6.18 & 8.87 & 15,010 & $10,080.3$ & .502 & .245 \\
\hline 250 & 08145000 & Brady Creek at Brady, Texas & $31^{\circ} 08^{\prime} 17^{\prime \prime}$ & $99^{\circ} 20^{\prime} 05^{\prime \prime}$ & $\mathrm{W}$ & 15 & 588 & 6.65 & 10.70 & 7,739 & $4,207.5$ & .568 & .450 \\
\hline 251 & 08146000 & San Saba River at San Saba, Texas & $31^{\circ} 12^{\prime} 47^{\prime \prime}$ & $98^{\circ} 43^{\prime} 09^{\prime \prime}$ & $\mathrm{W}$ & 47 & 3,039 & 8.03 & 8.02 & 22,236 & $13,288.1$ & .532 & .324 \\
\hline 252 & 08147000 & Colorado River near San Saba, Texas & $31^{\circ} 13^{\prime} 04^{\prime \prime}$ & $98^{\circ} 33^{\prime} 51^{\prime \prime}$ & $\mathrm{W}$ & 35 & 19,819 & 7.49 & 3.52 & 46,023 & $21,062.1$ & .562 & .366 \\
\hline 253 & 08148500 & North Llano River near Junction, Texas & $30^{\circ} 31^{\prime} 06^{\prime \prime}$ & $99^{\circ} 48^{\prime} 39^{\prime \prime}$ & $\mathrm{W}$ & 62 & 914 & 3.27 & 11.87 & 21,205 & $13,111.2$ & .401 & .109 \\
\hline 254 & 08150000 & Llano River near Junction, Texas & $30^{\circ} 29^{\prime} 51^{\prime \prime}$ & $99^{\circ} 43^{\prime} 19^{\prime \prime}$ & $\mathrm{W}$ & 80 & 1,851 & 2.98 & 9.98 & 33,902 & $22,555.8$ & .500 & .237 \\
\hline 255 & 08150700 & Llano River near Mason, Texas & $30^{\circ} 39^{\prime} 38^{\prime \prime}$ & $99^{\circ} 06^{\prime} 32^{\prime \prime}$ & $\mathrm{w}$ & 28 & 3,242 & 5.06 & 8.79 & 44,814 & $27,348.7$ & .460 & .268 \\
\hline 256 & 08150800 & Beaver Creek near Mason, Texas & $30^{\circ} 38^{\prime} 36^{\prime \prime}$ & $99^{\circ} 05^{\prime} 44^{\prime \prime}$ & $\mathrm{W}$ & 34 & 215 & 5.35 & 25.74 & 9,849 & $5,920.1$ & .470 & .320 \\
\hline 257 & 08151000 & Llano River near Castell, Texas & $30^{\circ} 43^{\prime} 00^{\prime \prime}$ & $98^{\circ} 53^{\prime} 00^{\prime \prime}$ & $\mathrm{W}$ & 15 & 3,742 & 5.62 & 8.12 & 76,621 & $43,300.6$ & .510 & .361 \\
\hline 258 & 08151500 & Llano River at Llano, Texas & $30^{\circ} 45^{\prime} 04^{\prime \prime}$ & $98^{\circ} 40^{\prime} 10^{\prime \prime}$ & $\mathrm{W}$ & 58 & 4,192 & 6.09 & 8.24 & 51,103 & $29,326.1$ & .417 & .189 \\
\hline
\end{tabular}


Table 1. Streamflow statistics and basin characteristics for 367 USGS streamflow-gaging stations with at least 10 years of annual peak-streamflow data through 1997 (designated as natural by 10-percent criterion)—Continued

\begin{tabular}{|c|c|c|c|c|c|c|c|c|c|c|c|c|c|}
\hline \multirow{2}{*}{$\begin{array}{l}\text { Seq. } \\
\text { no. }\end{array}$} & \multirow{2}{*}{$\begin{array}{l}\text { USGS } \\
\text { station } \\
\text { no. } \\
\text { (pl. 1) }\end{array}$} & \multirow{2}{*}{ USGS station name } & \multirow{2}{*}{ Latitude } & \multirow{2}{*}{ Longitude } & \multirow{2}{*}{ Region } & \multirow{3}{*}{$\begin{array}{c}\begin{array}{c}\text { Years } \\
\text { of } \\
\text { natural } \\
\text { annual } \\
\text { peak } \\
\text { stream- } \\
\text { flow }\end{array} \\
29\end{array}$} & \multirow{3}{*}{$\begin{array}{c}\begin{array}{c}\text { Contri- } \\
\text { buting } \\
\text { drainage } \\
\text { area } \\
\left(\mathbf{m i}^{2}\right)\end{array} \\
346\end{array}$} & \multirow{3}{*}{$\begin{array}{c}\begin{array}{c}\text { Basin } \\
\text { shape } \\
\text { factor } \\
\text { (dimen- } \\
\text { sion- } \\
\text { less) }\end{array} \\
6.75\end{array}$} & \multirow{2}{*}{$\begin{array}{l}\text { Stream } \\
\text { slope } \\
(\mathrm{ft} / \mathrm{mi})\end{array}$} & \multicolumn{4}{|c|}{$\begin{array}{l}\text { L-moment statistics of annual } \\
\text { peak-streamflow data }\end{array}$} \\
\hline & & & & & & & & & & $\begin{array}{l}\text { Mean } \\
\left(\mathrm{ft}^{3} / \mathrm{s}\right)\end{array}$ & $\begin{array}{c}\text { L-scale } \\
\left(\mathrm{ft}^{3} / \mathrm{s}\right)\end{array}$ & L-skew & L-kurtosis \\
\hline 259 & 08152000 & Sandy Creek near Kingsland, Texas & $30^{\circ} 33^{\prime} 30^{\prime \prime}$ & $98^{\circ} 28^{\prime} 19^{\prime \prime}$ & $\mathrm{W}$ & & & & 24.84 & 14,333 & $8,022.8$ & 0.519 & 0.407 \\
\hline 260 & 08152900 & Pedernales River near Fredericksburg, Texas & $30^{\circ} 13^{\prime} 13^{\prime \prime}$ & $98^{\circ} 52^{\prime} 10^{\prime \prime}$ & $\mathrm{W}$ & 17 & 369 & 4.09 & 17.64 & 14,684 & $9,631.9$ & .495 & .228 \\
\hline 261 & 08153500 & Pedernales River near Johnson City, Texas & $30^{\circ} 17^{\prime} 30^{\prime \prime}$ & $98^{\circ} 23^{\prime} 57^{\prime \prime}$ & $\mathrm{W}$ & 58 & 901 & 7.05 & 14.57 & 42,773 & $24,821.7$ & .495 & .321 \\
\hline 262 & 08154000 & Pedernales River near Spicewood, Texas & $30^{\circ} 25^{\prime} 15^{\prime \prime}$ & $98^{\circ} 04^{\prime} 50^{\prime \prime}$ & $\mathrm{W}$ & 15 & 1,294 & 11.31 & 13.26 & 33,841 & $21,878.6$ & .544 & .307 \\
\hline 263 & 08154700 & Bull Creek at Loop 360 near Austin, Texas & $30^{\circ} 22^{\prime} 19^{\prime \prime}$ & $97^{\circ} 47^{\prime} 04^{\prime \prime}$ & $\mathrm{W}$ & 19 & 22.3 & 3.96 & 47.82 & 4,732 & $2,325.7$ & .308 & .156 \\
\hline 264 & 08155200 & Barton Creek at SH 71 near Oak Hill, Texas & $30^{\circ} 17^{\prime} 46^{\prime \prime}$ & $97^{\circ} 55^{\prime} 31^{\prime \prime}$ & $\mathrm{W}$ & 16 & 89.7 & 8.38 & 22.62 & 4,206 & $2,324.7$ & .266 & .060 \\
\hline 265 & 08155300 & Barton Creek at Loop 360 at Austin, Texas & $30^{\circ} 14^{\prime} 40^{\prime \prime}$ & $97^{\circ} 48^{\prime} 07^{\prime \prime}$ & $\mathrm{W}$ & 22 & 116 & 16.33 & 18.84 & 5,419 & $3,128.2$ & .305 & .043 \\
\hline 266 & 08158000 & Colorado River at Austin, Texas & $30^{\circ} 14^{\prime} 40^{\prime \prime}$ & $97^{\circ} 41^{\prime} 39^{\prime \prime}$ & $\mathrm{W}$ & 39 & 27,606 & 11.21 & 3.66 & 76,995 & $33,850.6$ & .499 & .349 \\
\hline 267 & 08158700 & Onion Creek near Driftwood, Texas & $30^{\circ} 04^{\prime} 59^{\prime \prime}$ & $98^{\circ} 00^{\prime} 29^{\prime \prime}$ & $\mathrm{W}$ & 16 & 124 & 8.25 & 15.59 & 4,640 & 2249.1 & .058 & -.143 \\
\hline 268 & 08158810 & Bear Creek below FM 1826 near Driftwood, Texas & $30^{\circ} 09^{\prime} 19^{\prime \prime}$ & $97^{\circ} 56^{\prime} 23^{\prime \prime}$ & $\mathrm{W}$ & 19 & 12.20 & 2.47 & 55.55 & 1,944 & $1,356.4$ & .526 & .275 \\
\hline 269 & 08158840 & Slaughter Creek at FM 1826 near Austin, Texas & $30^{\circ} 12^{\prime} 32^{\prime \prime}$ & $97^{\circ} 54^{\prime} 11^{\prime \prime}$ & $\mathrm{W}$ & 20 & 8.24 & 2.14 & 52.32 & 1,497 & 905.5 & .381 & .114 \\
\hline 270 & 08159150 & Wilbarger Creek near Pflugerville, Texas & $30^{\circ} 27^{\prime} 16^{\prime \prime}$ & $97^{\circ} 36^{\prime} 02^{\prime \prime}$ & $\mathrm{W}$ & 17 & 4.61 & 1.94 & 38.44 & 659 & 203.2 & .258 & .321 \\
\hline 271 & 08160000 & Dry Creek at Buescher Lake near Smithville, Texas & $30^{\circ} 02^{\prime} 32^{\prime \prime}$ & $97^{\circ} 09^{\prime} 34^{\prime \prime}$ & SE & 26 & 1.48 & 2.15 & 69.96 & 489 & 282.9 & .332 & .063 \\
\hline 272 & 08160800 & Redgate Creek near Columbus, Texas & $29^{\circ} 47^{\prime} 56^{\prime \prime}$ & $96^{\circ} 31^{\prime} 55^{\prime \prime}$ & SE & 36 & 17.3 & 3.35 & 18.11 & 2,084 & 711.4 & .168 & .060 \\
\hline 273 & 08161000 & Colorado River at Columbus, Texas & $29^{\circ} 42^{\prime} 22^{\prime \prime}$ & $96^{\circ} 32^{\prime} 12^{\prime \prime}$ & SE & 21 & 30,237 & 16.90 & 3.22 & 59,303 & $21,371.0$ & .334 & .279 \\
\hline 274 & 08162600 & Tres Palacios River near Midfield, Texas & $28^{\circ} 55^{\prime} 40^{\prime \prime}$ & $96^{\circ} 10^{\prime} 15^{\prime \prime}$ & SE & 27 & 145 & 6.44 & 3.33 & 6085 & $1,820.7$ & .225 & .202 \\
\hline 275 & 08163500 & Lavaca River at Hallettsville, Texas & $29^{\circ} 26^{\prime} 35^{\prime \prime}$ & $96^{\circ} 56^{\prime} 39^{\prime \prime}$ & $\mathrm{SE}$ & 58 & 108 & 5.89 & 12.18 & 13,332 & $7,219.7$ & .495 & .345 \\
\hline 276 & 08164000 & Lavaca River near Edna, Texas & $28^{\circ} 57^{\prime} 35^{\prime \prime}$ & $96^{\circ} 41^{\prime} 10^{\prime \prime}$ & SE & 59 & 817 & 8.97 & 5.44 & 17,935 & $8,884.8$ & .484 & .385 \\
\hline 277 & 08164300 & Navidad River near Hallettsville, Texas & $29^{\circ} 28^{\prime} 00^{\prime \prime}$ & $96^{\circ} 48^{\prime} 45^{\prime \prime}$ & SE & 36 & 332 & 4.19 & 8.35 & 12,829 & $5,650.6$ & .276 & .183 \\
\hline 278 & 08164450 & Sandy Creek near Louise, Texas & $29^{\circ} 09^{\prime} 34^{\prime \prime}$ & $96^{\circ} 32^{\prime} 47^{\prime \prime}$ & SE & 20 & 289 & 10.65 & 5.97 & 6,934 & $2,756.2$ & .309 & .214 \\
\hline 279 & 08164500 & Navidad River near Ganado, Texas & $29^{\circ} 01^{\prime} 32^{\prime \prime}$ & $96^{\circ} 33^{\prime} 08^{\prime \prime}$ & SE & 42 & 826 & 10.40 & 4.78 & 18,854 & $8,430.8$ & .430 & .303 \\
\hline 280 & 08164503 & West Mustang Creek near Ganado, Texas & $29^{\circ} 04^{\prime} 17^{\prime \prime}$ & $96^{\circ} 28^{\prime} 01^{\prime \prime}$ & SE & 20 & 178 & 8.56 & 2.77 & 5,383 & $2,249.6$ & .408 & .302 \\
\hline 281 & 08164600 & Garcitas Creek near Inez, Texas & $28^{\circ} 53^{\prime} 28^{\prime \prime}$ & $96^{\circ} 49^{\prime} 08^{\prime \prime}$ & SE & 27 & 91.7 & 6.81 & 6.83 & 6,779 & $3,153.1$ & .312 & .125 \\
\hline 282 & 08164800 & Placedo Creek near Placedo, Texas & $28^{\circ} 43^{\prime} 30^{\prime \prime}$ & $96^{\circ} 46^{\prime} 07^{\prime \prime}$ & $\mathrm{SE}$ & 27 & 68.3 & 6.81 & 4.72 & 7,088 & $2,606.8$ & .187 & .112 \\
\hline 283 & 08165300 & North Fork Guadalupe River near Hunt, Texas & $30^{\circ} 03^{\prime} 36^{\prime \prime}$ & $99^{\circ} 23^{\prime} 40^{\prime \prime}$ & $\mathrm{W}$ & 30 & 168 & 4.53 & 20.35 & 13,842 & $8,687.9$ & .371 & .079 \\
\hline 284 & 08165500 & Guadalupe River at Hunt, Texas & $30^{\circ} 04^{\prime} 08^{\prime \prime}$ & $99^{\circ} 19^{\prime} 23^{\prime \prime}$ & $\mathrm{W}$ & 32 & 288 & 3.98 & 19.10 & 18,402 & $11,605.3$ & .441 & .191 \\
\hline 285 & 08166000 & Johnson Creek near Ingram, Texas & $30^{\circ} 06^{\prime} 00^{\prime \prime}$ & $99^{\circ} 16^{\prime} 58^{\prime \prime}$ & $\mathrm{W}$ & 50 & 114 & 3.44 & 25.03 & 8,663 & $6,880.0$ & .699 & .475 \\
\hline 286 & 08166200 & Guadalupe River at Kerrville, Texas & $30^{\circ} 03^{\prime} 09^{\prime \prime}$ & $99^{\circ} 09^{\prime} 54^{\prime \prime}$ & $\mathrm{W}$ & 12 & 510 & 3.85 & 16.73 & 27,540 & $19,533.4$ & .561 & .291 \\
\hline 287 & 08167000 & Guadalupe River at Comfort, Texas & $29^{\circ} 58^{\prime} 10^{\prime \prime}$ & $98^{\circ} 53^{\prime} 33^{\prime \prime}$ & $\mathrm{W}$ & 68 & 839 & 5.65 & 15.01 & 27,708 & $17,848.0$ & .534 & .310 \\
\hline
\end{tabular}


$\omega_{\infty} \quad$ Table 1. Streamflow statistics and basin characteristics for 367 USGS streamflow-gaging stations with at least 10 years of annual peak-streamflow data through 1997 (designated as natural by 10-percent criterion)-Continued

\begin{tabular}{|c|c|c|c|c|c|c|c|c|c|c|c|c|c|}
\hline \multirow{2}{*}{$\begin{array}{l}\text { Seq. } \\
\text { no. }\end{array}$} & \multirow{2}{*}{$\begin{array}{l}\text { USGS } \\
\text { station } \\
\text { no. } \\
\text { (pl. 1) }\end{array}$} & \multirow{2}{*}{ USGS station name } & \multirow{2}{*}{ Latitude } & \multirow{2}{*}{ Longitude } & \multirow{2}{*}{ Region } & \multirow{3}{*}{$\begin{array}{c}\begin{array}{c}\text { Years } \\
\text { of } \\
\text { natural } \\
\text { annual } \\
\text { peak } \\
\text { stream- } \\
\text { flow }\end{array} \\
75\end{array}$} & \multirow{3}{*}{$\begin{array}{c}\begin{array}{c}\text { Contri- } \\
\text { buting } \\
\text { drainage } \\
\text { area } \\
\left(\mathbf{m i}^{2}\right)\end{array} \\
1,315\end{array}$} & \multirow{3}{*}{$\begin{array}{c}\begin{array}{c}\text { Basin } \\
\text { shape } \\
\text { factor } \\
\text { (dimen- } \\
\text { sion- } \\
\text { less) }\end{array} \\
13.10\end{array}$} & \multirow{2}{*}{$\begin{array}{c}\text { Stream } \\
\text { slope } \\
(\mathrm{ft} / \mathrm{mi})\end{array}$} & \multicolumn{4}{|c|}{$\begin{array}{l}\text { L-moment statistics of annual } \\
\text { peak-streamflow data }\end{array}$} \\
\hline & & & & & & & & & & $\begin{array}{l}\text { Mean } \\
\left(\mathrm{ft}^{3} / \mathrm{s}\right)\end{array}$ & $\begin{array}{c}\text { L-scale } \\
\left(\mathrm{ft}^{3} / \mathrm{s}\right)\end{array}$ & L-skew & L-kurtosis \\
\hline 288 & 08167500 & Guadalupe River near Spring Branch, Texas & $29^{\circ} 51^{\prime} 38^{\prime \prime}$ & $98^{\circ} 22^{\prime} 58^{\prime \prime}$ & $\mathrm{W}$ & & & & 10.85 & 23,625 & $13,781.0$ & 0.515 & 0.289 \\
\hline 289 & 08167600 & Rebecca Creek near Spring Branch, Texas & $29^{\circ} 55^{\prime} 06^{\prime \prime}$ & $98^{\circ} 22^{\prime} 10^{\prime \prime}$ & $\mathrm{W}$ & 14 & 10.9 & 2.84 & 77.79 & 2,920 & $1,737.3$ & .283 & .025 \\
\hline 290 & 08168500 & Guadalupe River above Comal River at New Braunfels, Texas & $29^{\circ} 42^{\prime} 53^{\prime \prime}$ & $98^{\circ} 06^{\prime} 35^{\prime \prime}$ & $\mathrm{W}$ & 35 & 1,518 & 20.81 & 9.86 & 19,564 & $11,732.4$ & .519 & .288 \\
\hline 291 & 08169500 & Guadalupe River at New Braunfels, Texas & $29^{\circ} 41^{\prime} 52^{\prime \prime}$ & $98^{\circ} 06^{\prime} 23^{\prime \prime}$ & $\mathrm{W}$ & 13 & 1,652 & 19.52 & 9.98 & 21,289 & $11,349.1$ & .277 & -.040 \\
\hline 292 & 08171000 & Blanco River at Wimberley, Texas & $29^{\circ} 59^{\prime} 39^{\prime \prime}$ & $98^{\circ} 05^{\prime} 19^{\prime \prime}$ & $\mathrm{w}$ & 70 & 355 & 10.27 & 19.02 & 16,124 & $9,737.7$ & .479 & .295 \\
\hline 293 & 08171300 & Blanco River near Kyle, Texas & $29^{\circ} 58^{\prime} 45^{\prime \prime}$ & $97^{\circ} 54^{\prime} 35^{\prime \prime}$ & $\mathrm{W}$ & 41 & 412 & 14.42 & 17.11 & 18,287 & $10,293.3$ & .373 & .188 \\
\hline 294 & 08172000 & San Marcos River at Luling, Texas & $29^{\circ} 39^{\prime} 54^{\prime \prime}$ & $97^{\circ} 38^{\prime} 59^{\prime \prime}$ & SE & 44 & 838 & 18.43 & 13.07 & 16,318 & $8,049.7$ & .343 & .102 \\
\hline 295 & 08173000 & Plum Creek near Luling, Texas & $29^{\circ} 41^{\prime} 58^{\prime \prime}$ & $97^{\circ} 36^{\prime} 12^{\prime \prime}$ & SE & 34 & 309 & 5.79 & 12.56 & 12,376 & $7,169.8$ & .480 & .235 \\
\hline 296 & 08173500 & San Marcos River at Ottine, Texas & $29^{\circ} 35^{\prime} 36^{\prime \prime}$ & $97^{\circ} 35^{\prime} 22^{\prime \prime}$ & SE & 28 & 1,249 & 15.05 & 12.11 & 32,006 & $20,904.5$ & .626 & .423 \\
\hline 297 & 08174600 & Peach Creek below Dilworth, Texas & $29^{\circ} 28^{\prime} 26^{\prime \prime}$ & $97^{\circ} 18^{\prime} 59^{\prime \prime}$ & SE & 19 & 460 & 5.50 & 7.83 & 13,357 & $7,146.0$ & .528 & .468 \\
\hline 298 & 08175000 & Sandies Creek near Westhoff, Texas & $29^{\circ} 12^{\prime} 54^{\prime \prime}$ & $97^{\circ} 26 ' 57^{\prime \prime}$ & $\mathrm{SE}$ & 41 & 549 & 5.42 & 8.30 & 10,410 & $7,161.0$ & .610 & .396 \\
\hline 299 & 08176000 & Guadalupe River below Cuero, Texas & $29^{\circ} 03^{\prime} 05^{\prime \prime}$ & $97^{\circ} 15^{\prime} 52^{\prime \prime}$ & SE & 23 & 4,923 & 25.22 & 6.37 & 26,933 & $14,414.7$ & .436 & .123 \\
\hline 300 & 08176500 & Guadalupe River at Victoria, Texas & $28^{\circ} 47^{\prime} 34^{\prime \prime}$ & $97^{\circ} 00^{\prime} 46^{\prime \prime}$ & $\mathrm{SE}$ & 27 & 5,198 & 30.15 & 5.83 & 30,832 & $15,600.6$ & .422 & .262 \\
\hline 301 & 08176900 & Coleto Creek at Arnold Road near Schroeder, Texas & $28^{\circ} 51^{\prime} 41^{\prime \prime}$ & $97^{\circ} 13 ' 34 "$ & SE & 19 & 357 & 6.33 & 10.35 & 13,431 & $6,794.2$ & .232 & .096 \\
\hline 302 & 08177000 & Coleto Creek near Schroeder, Texas & $28^{\circ} 49^{\prime} 53^{\prime \prime}$ & $97^{\circ} 11^{\prime} 10^{\prime \prime}$ & SE & 31 & 369 & 7.04 & 8.30 & 18,880 & $10,767.6$ & .432 & .266 \\
\hline 303 & 08177300 & Perdido Creek at FM 622 near Fannin, Texas & $28^{\circ} 45^{\prime} 05^{\prime \prime}$ & $97^{\circ} 19^{\prime} 01^{\prime \prime}$ & SE & 19 & 28 & 2.63 & 17.21 & 6,896 & $4,131.0$ & .375 & .113 \\
\hline 304 & 08177500 & Coleto Creek near Victoria, Texas & $28^{\circ} 43^{\prime} 51^{\prime \prime}$ & $97^{\circ} 08^{\prime} 18^{\prime \prime}$ & SE & 18 & 514 & 7.32 & 7.65 & 17,896 & $10,665.2$ & .503 & .309 \\
\hline 305 & 08178500 & San Pedro Creek at Furnish Street at San Antonio, Texas & $29^{\circ} 24^{\prime} 22^{\prime \prime}$ & $98^{\circ} 30^{\prime} 38^{\prime \prime}$ & $\mathrm{W}$ & 14 & 2.64 & 84.89 & 29.81 & 738 & 273.0 & .190 & .240 \\
\hline 306 & 08178640 & West Elm Creek at San Antonio, Texas & $29^{\circ} 37^{\prime} 23^{\prime \prime}$ & $98^{\circ} 26^{\prime} 29^{\prime \prime}$ & $\mathrm{W}$ & 13 & 2.45 & 2.58 & 82.13 & 387 & 136.1 & .102 & .160 \\
\hline 307 & 08178880 & Medina River at Bandera, Texas & $29^{\circ} 43^{\prime} 26^{\prime \prime}$ & $99^{\circ} 04^{\prime} 13^{\prime \prime}$ & $\mathrm{W}$ & 15 & 427 & 6.28 & 19.83 & 12,918 & $9,259.9$ & .545 & .213 \\
\hline 308 & 08179000 & Medina River near Pipe Creek, Texas & $29^{\circ} 40^{\prime} 31^{\prime \prime}$ & $98^{\circ} 58^{\prime} 33^{\prime \prime}$ & $\mathrm{W}$ & 41 & 474 & 8.73 & 17.49 & 22,775 & $14,585.5$ & .613 & .468 \\
\hline 309 & 08179100 & Red Bluff Creek near Pipe Creek, Texas & $29^{\circ} 40^{\prime} 51^{\prime \prime}$ & $98^{\circ} 57^{\prime} 19^{\prime \prime}$ & $\mathrm{W}$ & 25 & 56.3 & 3.14 & 48.84 & 5506 & 3672.2 & .551 & .394 \\
\hline 310 & 08181000 & Leon Creek trib. at FM 1604 at San Antonio, Texas & $29^{\circ} 35^{\prime} 14^{\prime \prime}$ & $98^{\circ} 37^{\prime} 40^{\prime \prime}$ & $\mathrm{W}$ & 12 & 5.57 & 1.85 & 71.50 & 467 & 318.7 & .431 & .073 \\
\hline 311 & 08181400 & Helotes Creek at Helotes, Texas & $29^{\circ} 34^{\prime} 42^{\prime \prime}$ & $98^{\circ} 41^{\prime 2} 29^{\prime \prime}$ & $\mathrm{W}$ & 29 & 15 & 5.09 & 56.70 & 1,543 & $1,006.5$ & .444 & .185 \\
\hline 312 & 08182400 & Calaveras Creek Subwatershed No. 6 near Elmendorf, Texas & $29^{\circ} 22^{\prime} 49^{\prime \prime}$ & $98^{\circ} 17^{\prime} 33^{\prime \prime}$ & $\mathrm{W}$ & 21 & 7.01 & 2.24 & 32.31 & 1,734 & 869.0 & .286 & .011 \\
\hline 313 & 08183900 & Cibolo Creek near Boerne, Texas & $29^{\circ} 46^{\prime} 26^{\prime \prime}$ & $98^{\circ} 41^{\prime} 50^{\prime \prime}$ & $\mathrm{W}$ & 15 & 68.4 & 4.59 & 35.38 & 8,214 & $4,788.4$ & .431 & .245 \\
\hline 314 & 08184000 & Cibolo Creek near Bulverde, Texas & $29^{\circ} 43^{\prime} 33^{\prime \prime}$ & $98^{\circ} 25^{\prime} 37^{\prime \prime}$ & $\mathrm{W}$ & 20 & 198 & 9.25 & 22.22 & 5,699 & $3,688.5$ & .372 & .035 \\
\hline 315 & 08185000 & Cibolo Creek at Selma, Texas & $29^{\circ} 35^{\prime} 38^{\prime \prime}$ & $98^{\circ} 18^{\prime} 39^{\prime \prime}$ & $\mathrm{W}$ & 35 & 274 & 16.45 & 18.32 & 10,380 & $7,568.6$ & .559 & .279 \\
\hline 316 & 08186000 & Cibolo Creek near Falls City, Texas & $29^{\circ} 00^{\prime} 50^{\prime \prime}$ & $97^{\circ} 55^{\prime} 48^{\prime \prime}$ & SE & 67 & 827 & 24.66 & 11.90 & 9,939 & $4,359.0$ & .303 & .168 \\
\hline
\end{tabular}


Table 1. Streamflow statistics and basin characteristics for 367 USGS streamflow-gaging stations with at least 10 years of annual peak-streamflow data through 1997 (designated as natural by 10-percent criterion)—Continued

\begin{tabular}{|c|c|c|c|c|c|c|c|c|c|c|c|c|c|}
\hline \multirow{2}{*}{$\begin{array}{l}\text { Seq. } \\
\text { no. }\end{array}$} & \multirow{2}{*}{$\begin{array}{c}\text { USGS } \\
\text { station } \\
\text { no. } \\
\text { (pl. 1) }\end{array}$} & \multirow{2}{*}{ USGS station name } & \multirow{2}{*}{ Latitude } & \multirow{2}{*}{ Longitude } & \multirow{2}{*}{ Region } & \multirow{3}{*}{$\begin{array}{c}\begin{array}{c}\text { Years } \\
\text { of } \\
\text { natural } \\
\text { annual } \\
\text { peak }\end{array} \\
\text { stream- } \\
\text { flow }\end{array}$} & \multirow{3}{*}{$\begin{array}{c}\begin{array}{c}\text { Contri- } \\
\text { buting } \\
\text { drainage } \\
\text { area } \\
\left(\mathbf{m i}^{2}\right)\end{array} \\
239\end{array}$} & \multirow{3}{*}{$\begin{array}{c}\begin{array}{c}\text { Basin } \\
\text { shape } \\
\text { factor } \\
\text { (dimen- } \\
\text { sion- } \\
\text { less) }\end{array} \\
13.17\end{array}$} & \multirow{2}{*}{$\begin{array}{l}\text { Stream } \\
\text { slope } \\
(\mathrm{ft} / \mathrm{mi})\end{array}$} & \multicolumn{4}{|c|}{$\begin{array}{l}\text { L-moment statistics of annual } \\
\text { peak-streamflow data }\end{array}$} \\
\hline & & & & & & & & & & $\begin{array}{l}\text { Mean } \\
\left(\mathrm{ft}^{3} / \mathrm{s}\right)\end{array}$ & $\begin{array}{c}\text { L-scale } \\
\left(\mathrm{ft}^{3} / \mathrm{s}\right)\end{array}$ & L-skew & L-kurtosis \\
\hline 317 & 08186500 & Ecleto Creek near Runge, Texas & $28^{\circ} 55^{\prime} 12^{\prime \prime}$ & $97^{\circ} 46^{\prime} 19^{\prime \prime}$ & SE & & & & 7.66 & 7,075 & $5,326.4$ & 0.779 & 0.656 \\
\hline 318 & 08187900 & Escondido Creek Subwatershed No. 11 near Kenedy, Texas & $28^{\circ} 51^{\prime} 39^{\prime \prime}$ & $97^{\circ} 50^{\prime} 39^{\prime \prime}$ & $\mathrm{SE}$ & 20 & 8.43 & 2.10 & 30.39 & 1,760 & $1,374.0$ & .737 & .597 \\
\hline 319 & 08189200 & Copano Creek near Refugio, Texas & $28^{\circ} 18^{\prime} 12^{\prime \prime}$ & $97^{\circ} 06^{\prime} 44^{\prime \prime}$ & $\mathrm{SE}$ & 27 & 87.8 & 8.68 & 4.40 & 1,482 & 764.7 & .341 & .162 \\
\hline 320 & 08189300 & Medio Creek near Beeville, Texas & $28^{\circ} 28^{\prime} 58^{\prime \prime}$ & $97^{\circ} 39^{\prime} 23^{\prime \prime}$ & $\mathrm{SE}$ & 16 & 204 & 7.16 & 8.59 & 8,310 & $7,036.2$ & .894 & .894 \\
\hline 321 & 08189500 & Mission River at Refugio, Texas & $28^{\circ} 17^{\prime} 30^{\prime \prime}$ & $97^{\circ} 16^{\prime} 44^{\prime \prime}$ & $\mathrm{SE}$ & 58 & 690 & 9.31 & 5.98 & 10,026 & $5,925.1$ & .551 & .411 \\
\hline 322 & 08189700 & Aransas River near Skidmore, Texas & $28^{\circ} 16^{\prime} 56^{\prime \prime}$ & $97^{\circ} 37^{\prime} 14^{\prime \prime}$ & SE & 31 & 247 & 4.10 & 9.59 & 9,206 & $6,564.7$ & 688 & .545 \\
\hline 323 & 08189800 & Chiltipin Creek at Sinton, Texas & $28^{\circ} 02^{\prime} 48^{\prime \prime}$ & $97^{\circ} 30^{\prime} 13^{\prime \prime}$ & SE & 21 & 128 & 1.67 & 5.83 & 4,948 & $2,899.2$ & .486 & .368 \\
\hline 324 & 08190000 & Nueces River at Laguna, Texas & $29^{\circ} 25^{\prime} 42^{\prime \prime}$ & $99^{\circ} 59^{\prime} 49^{\prime \prime}$ & $\mathrm{W}$ & 75 & 737 & 5.80 & 17.21 & 33,539 & $24,031.2$ & .595 & .350 \\
\hline 325 & 08190500 & West Nueces River near Brackettville, Texas & $29^{\circ} 28^{\prime} 21^{\prime \prime}$ & $100^{\circ} 14^{\prime} 10^{\prime \prime}$ & $\mathrm{W}$ & 51 & 694 & 6.04 & 15.15 & 24,488 & $18,540.0$ & .611 & .373 \\
\hline 326 & 08192000 & Nueces River below Uvalde, Texas & $29^{\circ} 07^{\prime} 25^{\prime \prime}$ & $99^{\circ} 53^{\prime} 40^{\prime \prime}$ & $\mathrm{W}$ & 70 & 1,861 & 5.12 & 15.62 & 40,501 & $30,428.9$ & .634 & .415 \\
\hline 327 & 08194000 & Nueces River at Cotulla, Texas & $28^{\circ} 25^{\prime} 34^{\prime \prime}$ & $99^{\circ} 14^{\prime} 23^{\prime \prime}$ & $\mathrm{W}$ & 25 & 5,171 & 8.34 & 9.15 & 14,497 & $8,166.8$ & .541 & .337 \\
\hline 328 & 08194200 & San Casimiro Creek near Freer, Texas & $27^{\circ} 57^{\prime} 53^{\prime \prime}$ & $98^{\circ} 58^{\prime} 00^{\prime \prime}$ & $\mathrm{W}$ & 36 & 469 & 3.86 & 9.03 & 7,206 & $5,243.2$ & .730 & .548 \\
\hline 329 & 08194600 & Nueces River at Simmons, Texas & $28^{\circ} 25^{\prime} 16^{\prime \prime}$ & $98^{\circ} 17^{\prime} 03^{\prime \prime}$ & $\mathrm{W}$ & 13 & 8,561 & 10.88 & 7.08 & 15,830 & $9,798.7$ & .561 & .303 \\
\hline 330 & 08195000 & Frio River at Concan, Texas & $29^{\circ} 29^{\prime} 18^{\prime \prime}$ & $99^{\circ} 42^{\prime} 16^{\prime \prime}$ & $\mathrm{W}$ & 72 & 389 & 6.34 & 22.73 & 20,293 & $13,248.8$ & .493 & .266 \\
\hline 331 & 08196000 & Dry Frio River near Reagan Wells, Texas & $29^{\circ} 30^{\prime} 16^{\prime \prime}$ & $99^{\circ} 462^{\prime \prime}$ & $\mathrm{W}$ & 45 & 126 & 10.00 & 26.70 & 10,723 & $7,373.9$ & .558 & .376 \\
\hline 332 & 08197500 & Frio River below Dry Frio River near Uvalde, Texas & $29^{\circ} 14^{\prime} 44^{\prime \prime}$ & $99^{\circ} 40^{\prime} 27^{\prime \prime}$ & $\mathrm{W}$ & 46 & 631 & 7.19 & 20.94 & 18,834 & $12,146.3$ & .455 & .231 \\
\hline 333 & 08198000 & Sabinal River near Sabinal, Texas & $29^{\circ} 29^{\prime} 35^{\prime \prime}$ & $99^{\circ} 29^{\prime} 49^{\prime \prime}$ & $\mathrm{W}$ & 55 & 206 & 6.00 & 28.28 & 9,774 & $5,569.0$ & .389 & .214 \\
\hline 334 & 08198500 & Sabinal River at Sabinal, Texas & $29^{\circ} 18^{\prime} 47^{\prime \prime}$ & $99^{\circ} 28^{\prime} 46^{\prime \prime}$ & $\mathrm{W}$ & 45 & 241 & 12.39 & 23.00 & 12,880 & $8,542.8$ & .480 & .258 \\
\hline 335 & 08200000 & Hondo Creek near Tarpley, Texas & $29^{\circ} 34^{\prime} 10^{\prime \prime}$ & $99^{\circ} 14^{\prime} 47^{\prime \prime}$ & $\mathrm{W}$ & 45 & 95.6 & 3.14 & 41.84 & 12,264 & $7,769.9$ & .531 & .336 \\
\hline 336 & 08200500 & Hondo Creek near Hondo, Texas & $29^{\circ} 27^{\prime} 05^{\prime \prime}$ & $99^{\circ} 11^{\prime} 07^{\prime \prime}$ & $\mathrm{W}$ & 12 & 132 & 6.75 & 30.44 & 12,331 & $8,678.2$ & .582 & .464 \\
\hline 337 & 08200700 & Hondo Creek at King Waterhole near Hondo, Texas & $29^{\circ} 23^{\prime} 26^{\prime \prime}$ & $99^{\circ} 09^{\prime} 04^{\prime \prime}$ & $\mathrm{W}$ & 37 & 149 & 8.67 & 27.47 & 11,618 & $7,507.3$ & .453 & .233 \\
\hline 338 & 08201500 & Seco Creek at Miller Ranch near Utopia, Texas & $29^{\circ} 34^{\prime} 23^{\prime \prime}$ & $99^{\circ} 24^{\prime} 10^{\prime \prime}$ & $\mathrm{W}$ & 35 & 45 & 4.88 & 40.29 & 7,848 & $5,367.0$ & .563 & .369 \\
\hline 339 & 08202500 & Seco Creek near D'Hanis, Texas & $29^{\circ} 29^{\prime} 20^{\prime \prime}$ & $99^{\circ} 23^{\prime} 16^{\prime \prime}$ & $\mathrm{W}$ & 12 & 87.4 & 5.49 & 34.59 & 9,175 & $7,504.0$ & .786 & .665 \\
\hline 340 & 08202700 & Seco Creek at Rowe Ranch near D'Hanis, Texas & $29^{\circ} 21^{\prime} 43^{\prime \prime}$ & $99^{\circ} 17^{\prime} 05^{\prime \prime}$ & $\mathrm{W}$ & 37 & 168 & 9.38 & 24.79 & 8,335 & $5,744.5$ & .486 & .205 \\
\hline 341 & 08205500 & Frio River near Derby, Texas & $28^{\circ} 44^{\prime} 11^{\prime \prime}$ & $99^{\circ} 08^{\prime} 40^{\prime \prime}$ & $\mathrm{W}$ & 82 & 3,429 & 5.74 & 13.22 & 12,324 & $8,399.9$ & .643 & .504 \\
\hline 342 & 08206600 & Frio River at Tilden, Texas & $28^{\circ} 28^{\prime} 02^{\prime \prime}$ & $98^{\circ} 32^{\prime} 50^{\prime \prime}$ & $\mathrm{W}$ & 19 & 4,493 & 9.36 & 10.11 & 6,297 & $3,535.6$ & .358 & .081 \\
\hline 343 & 08206700 & San Miguel Creek near Tilden, Texas & $28^{\circ} 35^{\prime} 14^{\prime \prime}$ & $98^{\circ} 32^{\prime} 44^{\prime \prime}$ & $\mathrm{W}$ & 34 & 783 & 9.27 & 8.05 & 5,766 & $2,786.5$ & .281 & .095 \\
\hline 344 & 08207000 & Frio River at Calliham, Texas & $28^{\circ} 29^{\prime} 31^{\prime \prime}$ & $98^{\circ} 20^{\prime} 47^{\prime \prime}$ & $\mathrm{W}$ & 52 & 5,491 & 8.92 & 9.64 & 11039 & 5626.1 & .486 & .348 \\
\hline 345 & 08208000 & Atascosa River at Whitsett, Texas & $28^{\circ} 37^{\prime} 18^{\prime \prime}$ & $98^{\circ} 17^{\prime} 02^{\prime \prime}$ & $\mathrm{W}$ & 67 & 1,171 & 7.26 & 6.33 & 9289 & 5590.2 & .563 & .398 \\
\hline
\end{tabular}


f Table 1. Streamflow statistics and basin characteristics for 367 USGS streamflow-gaging stations with at least 10 years of annual peak-streamflow data through 1997 (designated as natural by 10-percent criterion)-Continued

\begin{tabular}{|c|c|c|c|c|c|c|c|c|c|c|c|c|c|}
\hline \multirow{2}{*}{$\begin{array}{l}\text { Seq. } \\
\text { no. }\end{array}$} & \multirow{2}{*}{$\begin{array}{c}\text { USGS } \\
\text { station } \\
\text { no. } \\
\text { (pl. 1) }\end{array}$} & \multirow{2}{*}{ USGS station name } & \multirow{2}{*}{ Latitude } & \multirow{2}{*}{ Longitude } & \multirow{2}{*}{ Region } & \multirow{3}{*}{$\begin{array}{c}\begin{array}{c}\text { Years } \\
\text { of } \\
\text { natural } \\
\text { annual } \\
\text { peak } \\
\text { stream- } \\
\text { flow }\end{array} \\
68\end{array}$} & \multirow{3}{*}{$\begin{array}{c}\begin{array}{c}\text { Contri- } \\
\text { buting } \\
\text { drainage } \\
\text { area } \\
\left(\mathrm{mi}^{2}\right)\end{array} \\
15,427\end{array}$} & \multirow{3}{*}{$\begin{array}{r}\begin{array}{c}\text { Basin } \\
\text { shape } \\
\text { factor } \\
\text { (dimen- } \\
\text { sion- } \\
\text { less) }\end{array} \\
6.40\end{array}$} & \multirow{2}{*}{$\begin{array}{c}\text { Stream } \\
\text { slope } \\
\text { (ft/mi) }\end{array}$} & \multicolumn{4}{|c|}{$\begin{array}{l}\text { L-moment statistics of annual } \\
\text { peak-streamflow data }\end{array}$} \\
\hline & & & & & & & & & & $\begin{array}{l}\text { Mean } \\
\left(\mathrm{ft}^{3} / \mathrm{s}\right)\end{array}$ & $\begin{array}{l}\text { L-scale } \\
\left(\mathrm{ft}^{3} / \mathrm{s}\right)\end{array}$ & L-skew & L-kurtosis \\
\hline 346 & 08210000 & Nueces River near Three Rivers, Texas & $28^{\circ} 25^{\prime} 38^{\prime \prime}$ & $98^{\circ} 10^{\prime} 40^{\prime \prime}$ & $\mathrm{W}$ & & & & 6.88 & 20,364 & $9,825.1$ & 0.455 & 0.288 \\
\hline 347 & 08210400 & Lagarto Creek near George West, Texas & $28^{\circ} 03^{\prime} 34^{\prime \prime}$ & $98^{\circ} 05^{\prime} 48^{\prime \prime}$ & $\mathrm{W}$ & 17 & 155 & 8.07 & 12.15 & 664 & 582.5 & .795 & .607 \\
\hline 348 & 08211520 & Oso Creek at Corpus Christi, Texas & $27^{\circ} 42^{\prime} 40^{\prime \prime}$ & $97^{\circ} 30^{\prime} 06^{\prime \prime}$ & $\mathrm{W}$ & 22 & 90.3 & 1.53 & 4.74 & 3,339 & $1,254.0$ & .299 & .287 \\
\hline 349 & 08212400 & Los Olmos Creek near Falfurrias, Texas & $27^{\circ} 15^{\prime} 51^{\prime \prime}$ & $98^{\circ} 08^{\prime} 08^{\prime \prime}$ & $\mathrm{W}$ & 17 & 476 & 8.60 & 11.18 & 1,082 & 755.5 & .543 & .226 \\
\hline 350 & 08365800 & Government Ditch at El Paso, Texas & $31^{\circ} 47^{\prime} 02^{\prime \prime}$ & $106^{\circ} 26^{\prime} 41^{\prime \prime}$ & $\mathrm{W}$ & 20 & 6.40 & 2.13 & 125.91 & 279 & 86.0 & -.007 & .084 \\
\hline 351 & 08374000 & Alamito Creek near Presidio, Texas & $29^{\circ} 31^{\prime} 15^{\prime \prime}$ & $104^{\circ} 17^{\prime} 40^{\prime \prime}$ & $\mathrm{W}$ & 52 & 1,504 & 6.86 & 40.69 & 9,510 & $3,339.8$ & .374 & .292 \\
\hline 352 & 08374500 & Terlingua Creek near Terlingua, Texas & $29^{\circ} 12^{\prime} 00^{\prime \prime}$ & $103^{\circ} 36^{\prime} 15^{\prime \prime}$ & $\mathrm{W}$ & 52 & 1,070 & 9.07 & 29.97 & 14,322 & $4,497.2$ & .117 & .084 \\
\hline 353 & 08376300 & Sanderson Canyon at Sanderson, Texas & $30^{\circ} 07^{\prime} 46^{\prime \prime}$ & $102^{\circ} 23^{\prime} 06^{\prime \prime}$ & $\mathrm{W}$ & 12 & 195 & 8.87 & 46.53 & 10,048 & $5,864.8$ & .393 & .197 \\
\hline 354 & 08408500 & Delaware River near Red Bluff, New Mexico & $32^{\circ} 01^{\prime} 12^{\prime \prime}$ & $104^{\circ} 03^{\prime} 00^{\prime \prime}$ & $\mathrm{W}$ & 60 & 689 & 5.26 & 39.50 & 7,469 & $5,032.2$ & .602 & .388 \\
\hline 355 & 08411500 & Salt Screwbean Draw near Orla, Texas & $31^{\circ} 52^{\prime} 40^{\prime \prime}$ & $103^{\circ} 56^{\prime} 50^{\prime \prime}$ & $\mathrm{W}$ & 15 & 464 & 4.30 & 30.86 & 5,074 & $3,440.8$ & .701 & .682 \\
\hline 356 & 08424500 & Madera Canyon near Toyahvale, Texas & $30^{\circ} 52^{\prime} 04^{\prime \prime}$ & $103^{\circ} 58^{\prime} 09^{\prime \prime}$ & $\mathrm{W}$ & 18 & 53.8 & 14.81 & 104.71 & 1,619 & 802.6 & .282 & .164 \\
\hline 357 & 08431700 & Limpia Creek above Fort Davis, Texas & $30^{\circ} 36^{\prime} 48^{\prime \prime}$ & $104^{\circ} 00^{\prime} 04^{\prime \prime}$ & $\mathrm{W}$ & 21 & 52.4 & 2.48 & 129.62 & 1,282 & 864.2 & .525 & .327 \\
\hline 358 & 08431800 & Limpia Creek below Fort Davis, Texas & $30^{\circ} 40^{\prime} 52^{\prime \prime}$ & $103^{\circ} 47^{\prime} 30^{\prime \prime}$ & $\mathrm{W}$ & 16 & 227 & 4.24 & 70.63 & 2,102 & 767.9 & .194 & .207 \\
\hline 359 & 08435800 & Coyanosa Draw near Fort Stockton, Texas & $31^{\circ} 02^{\prime} 27^{\prime \prime}$ & $103^{\circ} 08^{\prime} 15^{\prime \prime}$ & $\mathrm{W}$ & 14 & 1,182 & 6.73 & 26.90 & 2,052 & $1,459.3$ & .722 & .567 \\
\hline 360 & 08447020 & Independence Creek near Sheffield, Texas & $30^{\circ} 27^{\prime} 07^{\prime \prime}$ & $101^{\circ} 43^{\prime} 58^{\prime \prime}$ & $\mathrm{W}$ & 11 & 763 & 5.10 & 27.10 & 9,557 & $8,266.8$ & .843 & .769 \\
\hline 361 & 08447400 & Pecos River near Shumla, Texas & $29^{\circ} 50^{\prime} 00^{\prime \prime}$ & $101^{\circ} 23^{\prime} 00^{\prime \prime}$ & $\mathrm{W}$ & 55 & 35,162 & 6.81 & 4.09 & 50,081 & $34,579.8$ & .616 & .450 \\
\hline 362 & 08449000 & Devils River near Juno, Texas & $29^{\circ} 57^{\prime} 48^{\prime \prime}$ & $101^{\circ} 08^{\prime} 42^{\prime \prime}$ & $\mathrm{W}$ & 35 & 2,730 & 4.62 & 10.17 & 44,010 & $31,869.1$ & .590 & .363 \\
\hline 363 & 08449400 & Devils River at Pafford Crossing near Comstock, Texas & $29^{\circ} 40^{\prime} 35^{\prime \prime}$ & $101^{\circ} 00^{\prime} 00^{\prime \prime}$ & $\mathrm{W}$ & 16 & 3,961 & 5.38 & 9.95 & 62,838 & $41,090.0$ & .386 & .049 \\
\hline 364 & 08449500 & Devils River near Del Rio, Texas & $29^{\circ} 29^{\prime} 00^{\prime \prime}$ & $101^{\circ} 00^{\prime} 00^{\prime \prime}$ & $\mathrm{W}$ & 46 & 4,185 & 6.64 & 9.90 & 70,036 & $53,594.4$ & .663 & .424 \\
\hline 365 & 08450500 & Devils River near Mouth at Del Rio, Texas & $29^{\circ} 28^{\prime} 10^{\prime \prime}$ & $101^{\circ} 03^{\prime} 25^{\prime \prime}$ & $\mathrm{W}$ & 13 & 4,305 & 6.76 & 9.67 & 43,802 & $22,039.9$ & .198 & .022 \\
\hline 366 & 08453000 & San Felipe Creek near Del Rio, Texas & $29^{\circ} 19^{\prime} 55^{\prime \prime}$ & $100^{\circ} 53^{\prime} 20^{\prime \prime}$ & $\mathrm{W}$ & 29 & 46 & 5.44 & 20.32 & 9,372 & $4,729.9$ & .426 & .338 \\
\hline 367 & 08455000 & Pinto Creek near Del Rio, Texas & $29^{\circ} 08^{\prime} 45^{\prime \prime}$ & $100^{\circ} 43^{\prime} 05^{\prime \prime}$ & W & 54 & 249 & 7.85 & 17.43 & 12,031 & $8,917.1$ & .703 & .557 \\
\hline
\end{tabular}


Table 2. Static periods of regulation suitable for comparative analysis for 96 USGS streamflow-gaging stations with at least 10 years of annual peak-streamflow data through 1997 (designated as regulated by 10-percent criterion)

[Total period of station record analyzed was divided into number of static periods of regulation minus 1. Seq., sequence; mi ${ }^{2}$, square miles; $\mathrm{W}$, west; Max, representative maximum capacity (acre-feet) for corresponding static period of regulation; Norm, representative normal capacity (acre-feet) for corresponding static period of regulation; --, static period not applicable; NE, northeast; SE, southeast]

\begin{tabular}{|c|c|c|c|c|c|c|c|c|c|c|c|}
\hline $\begin{array}{l}\text { Seq. } \\
\text { no. }\end{array}$ & $\begin{array}{l}\text { USGS } \\
\text { station } \\
\text { number } \\
\text { (pl. 1) }\end{array}$ & USGS station name & Latitude & Longitude & Region & $\begin{array}{l}\text { Contri- } \\
\text { buting } \\
\text { drainage } \\
\text { area } \\
\left(\mathrm{mi}^{2}\right)\end{array}$ & $\begin{array}{l}\text { Total } \\
\text { period of } \\
\text { station } \\
\text { record } \\
\text { analyzed }\end{array}$ & $\begin{array}{c}\text { Static or } \\
\text { acceptably } \\
\text { static period } \\
\text { of regulation } \\
\text { no. } 1\end{array}$ & $\begin{array}{c}\text { Static or } \\
\text { acceptably } \\
\text { static period } \\
\text { of regulation } \\
\text { no. } 2\end{array}$ & $\begin{array}{c}\text { Static or } \\
\text { acceptably } \\
\text { static period } \\
\text { of regulation } \\
\text { no. } 3\end{array}$ & $\begin{array}{c}\text { Static or } \\
\text { acceptably } \\
\text { static period } \\
\text { of regulation } \\
\text { no. } 4\end{array}$ \\
\hline 1 & 07228000 & Canadian River near Canadian, Texas & $35^{\circ} 56^{\prime} 06^{\prime \prime}$ & $100^{\circ} 22^{\prime} 13^{\prime \prime}$ & $\mathrm{W}$ & 18,178 & 1938-97 & $\begin{array}{l}\text { Period: } 1938-64 \\
\text { Max: } 15,000 \\
\text { Norm: } 12,000\end{array}$ & $\begin{array}{l}\text { Period: } 1965-97 \\
\text { Max: 2,463,000 } \\
\text { Norm: } 885,000\end{array}$ & -- & -- \\
\hline 2 & 07235000 & Wolf Creek at Lipscomb, Texas & $36^{\circ} 14^{\prime} 19^{\prime \prime}$ & $100^{\circ} 16^{\prime} 31^{\prime \prime}$ & $\mathrm{W}$ & 475 & 1939-97 & $\begin{array}{l}\text { Period: } 1939-69 \\
\text { Max: } 2,792 \\
\text { Norm: } 753\end{array}$ & $\begin{array}{l}\text { Period: } 1970-97 \\
\text { Max: } 3,877 \\
\text { Norm: } 1,342\end{array}$ & -- & -- \\
\hline 3 & 07297500 & $\begin{array}{l}\text { Prairie Dog Town Fork Red River near } \\
\text { Canyon, Texas }\end{array}$ & $35^{\circ} 00^{\prime} 38^{\prime \prime}$ & $101^{\circ} 53 \prime 29^{\prime \prime}$ & $\mathrm{W}$ & 711 & $1939-49$ & $\begin{array}{l}\text { Period: } 1939-49 \\
\text { Max: } 74,360 \\
\text { Norm: 5,199 }\end{array}$ & -- & -- & -- \\
\hline 4 & 07298200 & Tule Creek near Silverton, Texas & $34^{\circ} 32^{\prime} 36^{\prime \prime}$ & $101^{\circ} 25^{\prime} 46^{\prime \prime}$ & $\mathrm{W}$ & 190 & $1965-85$ & $\begin{array}{l}\text { Period: } 1965-73 \\
\text { Max: } 3,230 \\
\text { Norm: } 1,580\end{array}$ & $\begin{array}{l}\text { Period: } 1974-85 \\
\text { Max: } 73,700 \\
\text { Norm: } 48,270\end{array}$ & -- & -- \\
\hline 5 & 07299540 & $\begin{array}{l}\text { Prairie Dog Town Fork Red River near } \\
\text { Childress, Texas }\end{array}$ & $34^{\circ} 34^{\prime} 09^{\prime \prime}$ & $100^{\circ} 11^{\prime} 37^{\prime \prime}$ & $\mathrm{W}$ & 2,956 & 1965-97 & $\begin{array}{l}\text { Period: } 1965-73 \\
\text { Max: } 129,000 \\
\text { Norm: } 32,900\end{array}$ & $\begin{array}{l}\text { Period: 1974-97 } \\
\text { Max: 231,000 } \\
\text { Norm: 85,700 }\end{array}$ & -- & -- \\
\hline 6 & 07300000 & Salt Fork Red River near Wellington, Texas & $34^{\circ} 57^{\prime} 27^{\prime \prime}$ & $100^{\circ} 13^{\prime} 14^{\prime \prime}$ & $\mathrm{W}$ & 1,013 & 1953-97 & $\begin{array}{l}\text { Period: } 1953-67 \\
\text { Max: } 900 \\
\text { Norm: } 430\end{array}$ & $\begin{array}{l}\text { Period: } 1968-97 \\
\text { Max: } 102,000 \\
\text { Norm: } 60,400\end{array}$ & -- & -- \\
\hline 7 & 07301200 & McClellan Creek near McLean, Texas & $35^{\circ} 19^{\prime} 45^{\prime \prime}$ & $100^{\circ} 36^{\prime} 32^{\prime \prime}$ & $\mathrm{W}$ & 460 & 1968-97 & $\begin{array}{l}\text { Period: } 1968-78 \\
\text { Max: } 9,200 \\
\text { Norm: } 3,350\end{array}$ & $\begin{array}{l}\text { Period: } 1979-85 \\
\text { Max: } 33,900 \\
\text { Norm: } 4,760\end{array}$ & $\begin{array}{l}\text { Period: } 1986-97 \\
\text { Max: } 40,500 \\
\text { Norm: } 7,550\end{array}$ & -- \\
\hline 8 & 07314900 & Little Wichita River above Henrietta, Texas & $33^{\circ} 49^{\prime} 36^{\prime \prime}$ & $98^{\circ} 14^{\prime} 23^{\prime \prime}$ & $\mathrm{NE}$ & 1,037 & 1953-97 & $\begin{array}{l}\text { Period: } 1953-65 \\
\text { Max: } 219,000 \\
\text { Norm: } 114,400\end{array}$ & $\begin{array}{l}\text { Period: } 1966-97 \\
\text { Max: } 911,800 \\
\text { Norm: } 379,400\end{array}$ & -- & -- \\
\hline 9 & 07315500 & Red River near Terral, Oklahoma & $33^{\circ} 52^{\prime} 43^{\prime \prime}$ & $97^{\circ} 56^{\prime} 03^{\prime \prime}$ & $\mathrm{NE}$ & 28,723 & 1938-97 & $\begin{array}{l}\text { Period: } 1938-65 \\
\text { Max: } 1,693,800 \\
\text { Norm: } 470,100\end{array}$ & $\begin{array}{l}\text { Period: } 1966-97 \\
\text { Max: } 2,586,500 \\
\text { Norm: } 833,360\end{array}$ & -- & -- \\
\hline 10 & 07316000 & Red River near Gainesville, Texas & $33^{\circ} 43^{\prime} 40^{\prime \prime}$ & $97^{\circ} 09^{\prime} 35^{\prime \prime}$ & $\mathrm{NE}$ & 30,782 & 1936-97 & $\begin{array}{l}\text { Period: } 1936-65 \\
\text { Max: } 1,696,700 \\
\text { Norm: } 471,730\end{array}$ & $\begin{array}{l}\text { Period: } 1966-97 \\
\text { Max: } 2,725,500 \\
\text { Norm: } 887,300\end{array}$ & -- & -- \\
\hline
\end{tabular}


N Table 2. Static periods of regulation suitable for comparative analysis for 96 USGS streamflow-gaging stations with at least 10 years of annual peak-streamflow data through 1997 (designated as regulated by 10-percent criterion)-Continued

\begin{tabular}{|c|c|c|c|c|c|c|c|c|c|c|c|}
\hline $\begin{array}{l}\text { Seq. } \\
\text { no. }\end{array}$ & $\begin{array}{c}\text { USGS } \\
\text { station } \\
\text { number } \\
\text { (pl. 1) }\end{array}$ & USGS station name & Latitude & Longitude & Region & $\begin{array}{l}\text { Contri- } \\
\text { buting } \\
\text { drainage } \\
\text { area } \\
\left(\mathrm{mi}^{2}\right)\end{array}$ & $\begin{array}{l}\text { Total } \\
\text { period of } \\
\text { station } \\
\text { record } \\
\text { analyzed }\end{array}$ & $\begin{array}{c}\text { Static or } \\
\text { acceptably } \\
\text { static period } \\
\text { of regulation } \\
\text { no. } 1\end{array}$ & $\begin{array}{c}\text { Static or } \\
\text { acceptably } \\
\text { static period } \\
\text { of regulation } \\
\text { no. } 2\end{array}$ & $\begin{array}{c}\text { Static or } \\
\text { acceptably } \\
\text { static period } \\
\text { of regulation } \\
\text { no. } 3\end{array}$ & $\begin{array}{c}\text { Static or } \\
\text { acceptably } \\
\text { static period } \\
\text { of regulation } \\
\text { no. } 4\end{array}$ \\
\hline 11 & 07343500 & White Oak Creek near Talco, Texas & $33^{\circ} 19^{\prime} 20^{\prime \prime}$ & $95^{\circ} 05^{\prime} 33^{\prime \prime}$ & NE & 494 & $1950-97$ & $\begin{array}{l}\text { Period: } 1950-72 \\
\text { Max: 5,365 } \\
\text { Norm: } 2,541\end{array}$ & $\begin{array}{l}\text { Period: } 1973-97 \\
\text { Max: } 47,500 \\
\text { Norm: } 23,600\end{array}$ & -- & -- \\
\hline 12 & 07344500 & Big Cypress Creek near Pittsburg, Texas & $33^{\circ} 01^{\prime} 15^{\prime \prime}$ & $94^{\circ} 52^{\prime} 55^{\prime \prime}$ & $\mathrm{NE}$ & 366 & 1943-97 & $\begin{array}{l}\text { Period: } 1943-70 \\
\text { Max: } 8,678 \\
\text { Norm: } 4,831\end{array}$ & $\begin{array}{l}\text { Period: } 1978-97 \\
\text { Max: } 522,700 \\
\text { Norm: } 324,000\end{array}$ & -- & -- \\
\hline 13 & 07346000 & Big Cypress Creek near Jefferson, Texas & $32^{\circ} 44^{\prime} 58^{\prime \prime}$ & $94^{\circ} 29^{\prime} 55^{\prime \prime}$ & $\mathrm{NE}$ & 850 & 1913-97 & $\begin{array}{l}\text { Period: } 1913-58 \\
\text { Max: } 3,980 \\
\text { Norm: } 1,830\end{array}$ & $\begin{array}{l}\text { Period: } 1959-97 \\
\text { Max: 2,198,000 } \\
\text { Norm: 436,500 }\end{array}$ & -- & -- \\
\hline 14 & 08018500 & Sabine River near Mineola, Texas & $32^{\circ} 36^{\prime} 49^{\prime \prime}$ & $95^{\circ} 29^{\prime} 08^{\prime \prime}$ & $\mathrm{NE}$ & 1,357 & 1938-97 & $\begin{array}{l}\text { Period: } 1938-59 \\
\text { Max: } 23,000 \\
\text { Norm: } 14,700\end{array}$ & $\begin{array}{l}\text { Period: } 1960-97 \\
\text { Max: 1,719,900 } \\
\text { Norm: } 957,700\end{array}$ & -- & -- \\
\hline 15 & 08019000 & Lake Fork Creek near Quitman, Texas & $32^{\circ} 45^{\prime} 47^{\prime \prime}$ & $95^{\circ} 27^{\prime} 46^{\prime \prime}$ & $\mathrm{NE}$ & 585 & $1925-97$ & $\begin{array}{l}\text { Period: } 1925-61 \\
\text { Max: } 3,160 \\
\text { Norm: } 1,520\end{array}$ & $\begin{array}{l}\text { Period: } 1962-79 \\
\text { Max: } 65,500 \\
\text { Norm: } 12,500\end{array}$ & $\begin{array}{l}\text { Period: } 1980-97 \\
\text { Max: 1,122,500 } \\
\text { Norm: } 689,400\end{array}$ & -- \\
\hline 16 & 08019500 & Big Sandy Creek near Big Sandy, Texas & $32^{\circ} 36^{\prime} 14^{\prime \prime}$ & $95^{\circ} 05^{\prime} 29^{\prime \prime}$ & $\mathrm{NE}$ & 231 & 1939-97 & $\begin{array}{l}\text { Period: } 1939-61 \\
\text { Max: } 4,096 \\
\text { Norm: } 1,885\end{array}$ & $\begin{array}{l}\text { Period: } 1962-97 \\
\text { Max: } 87,140 \\
\text { Norm: } 29,980\end{array}$ & -- & -- \\
\hline 17 & 08020000 & Sabine River near Gladewater, Texas & $32^{\circ} 31^{\prime} 37^{\prime \prime}$ & $94^{\circ} 57^{\prime} 36^{\prime \prime}$ & NE & 2,791 & 1932-97 & $\begin{array}{l}\text { Period: } 1932-59 \\
\text { Max: } 61,000 \\
\text { Norm: } 34,700\end{array}$ & $\begin{array}{l}\text { Period: } 1960-79 \\
\text { Max: 1,899,500 } \\
\text { Norm: 101,6000 }\end{array}$ & $\begin{array}{l}\text { Period: } 1980-97 \\
\text { Max: } 2,977,000 \\
\text { Norm: } 1,702,000\end{array}$ & -- \\
\hline 18 & 08026000 & Sabine River near Burkeville, Texas & $31^{\circ} 03^{\prime} 50^{\prime \prime}$ & $93^{\circ} 31^{\prime} 10^{\prime \prime}$ & SE & 7,482 & 1956-97 & $\begin{array}{l}\text { Period: } 1968-79 \\
\text { Max: } 7,415,000 \\
\text { Norm: 5,698,000 }\end{array}$ & $\begin{array}{l}\text { Period: } 1980-97 \\
\text { Max: 8,534,000 } \\
\text { Norm: } 6,412,000\end{array}$ & -- & -- \\
\hline 19 & 08028500 & Sabine River near Bon Weir, Texas & $30^{\circ} 44^{\prime} 49^{\prime \prime}$ & $93^{\circ} 36^{\prime} 30^{\prime \prime}$ & $\mathrm{SE}$ & 8,229 & 1924-97 & $\begin{array}{l}\text { Period: } 1924-59 \\
\text { Max: } 148,000 \\
\text { Norm: } 87,300\end{array}$ & $\begin{array}{l}\text { Period: } 1968-79 \\
\text { Max: 7,415,000 } \\
\text { Norm: } 5,698,000\end{array}$ & $\begin{array}{l}\text { Period: } 1980-97 \\
\text { Max: } 8,534,000 \\
\text { Norm: } 6,412,000\end{array}$ & -- \\
\hline 20 & 08030500 & Sabine River near Ruliff, Texas & $30^{\circ} 18^{\prime} 13^{\prime \prime}$ & $93^{\circ} 44^{\prime} 37^{\prime \prime}$ & $\mathrm{SE}$ & 9,329 & 1908-97 & $\begin{array}{l}\text { Period: } 1908-59 \\
\text { Max: } 148,000 \\
\text { Norm: } 87,300\end{array}$ & $\begin{array}{l}\text { Period: } 1968-79 \\
\text { Max: } 7,416,000 \\
\text { Norm: } 5,699,000\end{array}$ & $\begin{array}{l}\text { Period: } 1980-97 \\
\text { Max: 8,535,000 } \\
\text { Norm: } 6,413,000\end{array}$ & -- \\
\hline 21 & 08032000 & Neches River near Neches, Texas & $31^{\circ} 53^{\prime} 32^{\prime \prime}$ & $95^{\circ} 25^{\prime} 50^{\prime \prime}$ & NE & 1,145 & 1939-97 & $\begin{array}{l}\text { Period: } 1939-70 \\
\text { Max: } 29,400 \\
\text { Norm: } 12,400\end{array}$ & $\begin{array}{l}\text { Period: } 1971-97 \\
\text { Max: } 1,146,000 \\
\text { Norm: } 468,700\end{array}$ & -- & -- \\
\hline
\end{tabular}


Table 2. Static periods of regulation suitable for comparative analysis for 96 USGS streamflow-gaging stations with at least 10 years of annual peak-streamflow data through 1997 (designated as regulated by 10-percent criterion)—Continued

\begin{tabular}{|c|c|c|c|c|c|c|c|c|c|c|c|}
\hline $\begin{array}{l}\text { Seq. } \\
\text { no. }\end{array}$ & $\begin{array}{l}\text { USGS } \\
\text { station } \\
\text { number } \\
\text { (pl. 1) }\end{array}$ & USGS station name & Latitude & Longitude & Region & $\begin{array}{l}\text { Contri- } \\
\text { buting } \\
\text { drainage } \\
\text { area } \\
\left(\mathrm{mi}^{2}\right)\end{array}$ & $\begin{array}{l}\text { Total } \\
\text { period of } \\
\text { station } \\
\text { record } \\
\text { analyzed }\end{array}$ & $\begin{array}{c}\text { Static or } \\
\text { acceptably } \\
\text { static period } \\
\text { of regulation } \\
\text { no. } 1\end{array}$ & $\begin{array}{c}\text { Static or } \\
\text { acceptably } \\
\text { static period } \\
\text { of regulation } \\
\text { no. } 2\end{array}$ & $\begin{array}{c}\text { Static or } \\
\text { acceptably } \\
\text { static period } \\
\text { of regulation } \\
\text { no. } 3\end{array}$ & $\begin{array}{c}\text { Static or } \\
\text { acceptably } \\
\text { static period } \\
\text { of regulation } \\
\text { no. } 4\end{array}$ \\
\hline 22 & 08034500 & Mud Creek near Jacksonville, Texas & $31^{\circ} 58^{\prime} 35^{\prime \prime}$ & $95^{\circ} 09^{\prime} 38^{\prime \prime}$ & $\mathrm{NE}$ & 376 & $1939-79$ & $\begin{array}{l}\text { Period: } 1939-48 \\
\text { Max: } 2,985 \\
\text { Norm: } 972\end{array}$ & $\begin{array}{l}\text { Period: } 1949-66 \\
\text { Max: } 88,900 \\
\text { Norm: } 43,500\end{array}$ & $\begin{array}{l}\text { Period: } 1967-79 \\
\text { Max: } 175,600 \\
\text { Norm: } 87,200\end{array}$ & -- \\
\hline 23 & 08037000 & Angelina River near Lufkin, Texas & $31^{\circ} 27^{\prime} 26^{\prime \prime}$ & $94^{\circ} 43^{\prime} 34^{\prime \prime}$ & $\mathrm{NE}$ & 1,600 & $1924-76$ & $\begin{array}{l}\text { Period: } 1924-48 \\
\text { Max: } 7,205 \\
\text { Norm: } 3,293\end{array}$ & $\begin{array}{l}\text { Period: } 1956-66 \\
\text { Max: } 177,400 \\
\text { Norm: } 75,400\end{array}$ & $\begin{array}{l}\text { Period: } 1967-76 \\
\text { Max: } 264,900 \\
\text { Norm: } 119,000\end{array}$ & -- \\
\hline 24 & 08041000 & Neches River at Evadale, Texas & $30^{\circ} 21^{\prime} 20^{\prime \prime}$ & $94^{\circ} 05^{\prime} 35^{\prime \prime}$ & SE & 7,951 & 1905-97 & $\begin{array}{l}\text { Period: } 1905-50 \\
\text { Max: } 24,200 \\
\text { Norm: } 8,669\end{array}$ & $\begin{array}{l}\text { Period: } 1951-64 \\
\text { Max: } 644,600 \\
\text { Norm: } 252,700\end{array}$ & $\begin{array}{l}\text { Period: } 1965-97 \\
\text { Max: } 8,434,000 \\
\text { Norm: } 3,682,000\end{array}$ & -- \\
\hline 25 & 08046000 & Clear Fork Trinity River near Aledo, Texas & $32^{\circ} 38^{\prime} 28^{\prime \prime}$ & $97^{\circ} 333^{\prime} 51^{\prime \prime}$ & $\mathrm{NE}$ & 251 & $1948-75$ & $\begin{array}{l}\text { Period: } 1948-56 \\
\text { Max: } 1,094 \\
\text { Norm: } 548\end{array}$ & $\begin{array}{l}\text { Period: } 1957-75 \\
\text { Max: } 81,260 \\
\text { Norm: } 26,460\end{array}$ & -- & -- \\
\hline 26 & 08047500 & Clear Fork Trinity River at Fort Worth, Texas & $32^{\circ} 43^{\prime} 56^{\prime \prime}$ & $97^{\circ} 21^{\prime} 31^{\prime \prime}$ & $\mathrm{NE}$ & 518 & $1924-97$ & $\begin{array}{l}\text { Period: } 1924-49 \\
\text { Max: } 1,631 \\
\text { Norm: } 973\end{array}$ & $\begin{array}{l}\text { Period: } 1957-97 \\
\text { Max: 500,000 } \\
\text { Norm: } 119,000\end{array}$ & -- & -- \\
\hline 27 & 08048000 & West Fork Trinity River at Fort Worth, Texas & $32^{\circ} 45^{\prime} 39^{\prime \prime}$ & $97^{\circ} 19^{\prime} 56^{\prime \prime}$ & $\mathrm{NE}$ & 2,615 & 1921-97 & $\begin{array}{l}\text { Period: } 1921-49 \\
\text { Max: 1,631 } \\
\text { Norm: } 973\end{array}$ & $\begin{array}{l}\text { Period: } 1957-97 \\
\text { Max: } 505,000 \\
\text { Norm: } 120,000\end{array}$ & -- & -- \\
\hline 28 & 08050100 & Mountain Creek at Grand Prairie, Texas & $32^{\circ} 44^{\prime} 51^{\prime \prime}$ & $96^{\circ} 55^{\prime} 32^{\prime \prime}$ & $\mathrm{NE}$ & 298 & $1961-97$ & $\begin{array}{l}\text { Period: } 1961-85 \\
\text { Max: } 82,010 \\
\text { Norm: } 41,970\end{array}$ & $\begin{array}{l}\text { Period: } 1986-97 \\
\text { Max: } 724,200 \\
\text { Norm: } 218,700\end{array}$ & -- & -- \\
\hline 29 & 08050500 & Elm Fork Trinity River near Sanger, Texas & $33^{\circ} 23^{\prime} 11^{\prime \prime}$ & $97^{\circ} 05^{\prime} 05^{\prime \prime}$ & $\mathrm{NE}$ & 381 & $1963-83$ & $\begin{array}{l}\text { Period: } 1963-83 \\
\text { Max: 44,900 } \\
\text { Norm: } 4,374\end{array}$ & -- & -- & -- \\
\hline 30 & 08051500 & Clear Creek near Sanger, Texas & $33^{\circ} 20^{\prime} 21^{\prime \prime}$ & $97^{\circ} 10^{\prime} 51^{\prime \prime}$ & $\mathrm{NE}$ & 295 & 1949-97 & $\begin{array}{l}\text { Period: 1949-59 } \\
\text { Max: } 0 \\
\text { Norm: } 0\end{array}$ & $\begin{array}{l}\text { Period: } 1970-97 \\
\text { Max: } 76,800 \\
\text { Norm: } 7,600\end{array}$ & -- & -- \\
\hline 31 & 08052700 & Little Elm Creek near Aubrey, Texas & $33^{\circ} 17^{\prime} 00^{\prime \prime}$ & $96^{\circ} 53^{\prime} 33^{\prime \prime}$ & $\mathrm{NE}$ & 75.5 & $1957-97$ & $\begin{array}{l}\text { Period: } 1957-65 \\
\text { Max: } 0 \\
\text { Norm: } 0\end{array}$ & $\begin{array}{l}\text { Period: } 1970-97 \\
\text { Max: } 20,600 \\
\text { Norm: } 2,265\end{array}$ & -- & -- \\
\hline 32 & 08053500 & Denton Creek near Justin, Texas & $33^{\circ} 07^{\prime} 08^{\prime \prime}$ & $97^{\circ} 17^{\prime} 25^{\prime \prime}$ & $\mathrm{NE}$ & 400 & $1950-97$ & $\begin{array}{l}\text { Period: } 1950-66 \\
\text { Max: } 2,451 \\
\text { Norm: } 847\end{array}$ & $\begin{array}{l}\text { Period: } 1972-97 \\
\text { Max: } 87,900 \\
\text { Norm: } 8,396\end{array}$ & -- & -- \\
\hline
\end{tabular}


\$ Table 2. Static periods of regulation suitable for comparative analysis for 96 USGS streamflow-gaging stations with at least 10 years of annual peak-streamflow data through 1997 (designated as regulated by 10-percent criterion)-Continued

\begin{tabular}{|c|c|c|c|c|c|c|c|c|c|c|c|}
\hline $\begin{array}{c}\text { Seq. } \\
\text { no. }\end{array}$ & $\begin{array}{c}\text { USGS } \\
\text { station } \\
\text { number } \\
\text { (pl. 1) }\end{array}$ & USGS station name & Latitude & Longitude & Region & $\begin{array}{c}\text { Contri- } \\
\text { buting } \\
\text { drainage } \\
\text { area } \\
\left(\mathrm{mi}^{2}\right)\end{array}$ & $\begin{array}{l}\text { Total } \\
\text { period of } \\
\text { station } \\
\text { record } \\
\text { analyzed }\end{array}$ & $\begin{array}{c}\text { Static or } \\
\text { acceptably } \\
\text { static period } \\
\text { of regulation } \\
\text { no. } 1\end{array}$ & $\begin{array}{c}\text { Static or } \\
\text { acceptably } \\
\text { static period } \\
\text { of regulation } \\
\text { no. } 2\end{array}$ & $\begin{array}{c}\text { Static or } \\
\text { acceptably } \\
\text { static period } \\
\text { of regulation } \\
\text { no. } 3\end{array}$ & $\begin{array}{c}\text { Static or } \\
\text { acceptably } \\
\text { static period } \\
\text { of regulation } \\
\text { no. } 4\end{array}$ \\
\hline 33 & 08057000 & Trinity River at Dallas, Texas & $32^{\circ} 46^{\prime} 29^{\prime \prime}$ & $96^{\circ} 49^{\prime} 18^{\prime \prime}$ & $\mathrm{NE}$ & 6,106 & 1904-97 & $\begin{array}{l}\text { Period: } 1904-30 \\
\text { Max: } 99,810 \\
\text { Norm: } 70,260\end{array}$ & $\begin{array}{l}\text { Period: } 1931-53 \\
\text { Max: } 1,778,000 \\
\text { Norm: } 688,900\end{array}$ & $\begin{array}{l}\text { Period: } 1954-85 \\
\text { Max: } 5,666,000 \\
\text { Norm: } 1,579,000\end{array}$ & $\begin{array}{l}\text { Period: } 1986-97 \\
\text { Max: } 8,308,000 \\
\text { Norm: } 2,609,000\end{array}$ \\
\hline 34 & 08059000 & East Fork Trinity River near McKinney, Texas & $33^{\circ} 12^{\prime} 13^{\prime \prime}$ & $96^{\circ} 35^{\prime} 44^{\prime \prime}$ & $\mathrm{NE}$ & 190 & $1955-74$ & $\begin{array}{l}\text { Period: } 1955-74 \\
\text { Max: } 23,560 \\
\text { Norm: } 3,644\end{array}$ & -- & -- & -- \\
\hline 35 & 08059500 & Sister Grove Creek near Princeton, Texas & $33^{\circ} 11^{\prime} 35^{\prime \prime}$ & $96^{\circ} 28^{\prime} 32^{\prime \prime}$ & $\mathrm{NE}$ & 113 & $1950-75$ & $\begin{array}{l}\text { Period: } 1960-75 \\
\text { Max: } 31,970 \\
\text { Norm: } 4,600\end{array}$ & -- & -- & -- \\
\hline 36 & 08062500 & Trinity River near Rosser, Texas & $32^{\circ} 25^{\prime} 35^{\prime \prime}$ & $96^{\circ} 27^{\prime} 46^{\prime \prime}$ & $\mathrm{NE}$ & 8,146 & 1939-97 & $\begin{array}{l}\text { Period: } 1939-52 \\
\text { Max: } 1,823,000 \\
\text { Norm: } 701,300\end{array}$ & $\begin{array}{l}\text { Period: } 1954-85 \\
\text { Max: 7,038,000 } \\
\text { Norm: } 230,800\end{array}$ & $\begin{array}{l}\text { Period: } 1986-97 \\
\text { Max: } 10,080,000 \\
\text { Norm: } 3,600,000\end{array}$ & -- \\
\hline 37 & 08062800 & Cedar Creek near Kemp, Texas & $32^{\circ} 30^{\prime} 18^{\prime \prime}$ & $96^{\circ} 06^{\prime} 57^{\prime \prime}$ & NE & 189 & $1963-87$ & $\begin{array}{l}\text { Period: } 1971-87 \\
\text { Max: } 56,080 \\
\text { Norm: } 11,220\end{array}$ & -- & -- & -- \\
\hline 38 & 08063500 & Richland Creek near Richland, Texas & $31^{\circ} 57^{\prime} 02^{\prime \prime}$ & $96^{\circ} 25^{\prime} 16^{\prime \prime}$ & $\mathrm{NE}$ & 734 & 1939-89 & $\begin{array}{l}\text { Period: } 1939-62 \\
\text { Max: } 986 \\
\text { Norm: } 613\end{array}$ & $\begin{array}{l}\text { Period: } 1963-89 \\
\text { Max: } 445,600 \\
\text { Norm: } 74,860\end{array}$ & -- & -- \\
\hline 39 & 08064500 & Chambers Creek near Corsicana, Texas & $32^{\circ} 06^{\prime} 29^{\prime \prime}$ & $96^{\circ} 22^{\prime} 14^{\prime \prime}$ & NE & 963 & 1939-84 & $\begin{array}{l}\text { Period: } 1939-59 \\
\text { Max: } 54,280 \\
\text { Norm: } 25,280\end{array}$ & $\begin{array}{l}\text { Period: } 1965-84 \\
\text { Max: } 495,300 \\
\text { Norm: } 101,800\end{array}$ & -- & -- \\
\hline 40 & 08065000 & Trinity River near Oakwood, Texas & $31^{\circ} 38^{\prime} 54^{\prime \prime}$ & $95^{\circ} 47^{\prime} 21^{\prime \prime}$ & $\mathrm{NE}$ & 12,833 & 1924-97 & $\begin{array}{l}\text { Period: } 1924-53 \\
\text { Max: } 1,886,000 \\
\text { Norm: } 723,000\end{array}$ & $\begin{array}{l}\text { Period: } 1954-65 \\
\text { Max: } 6,810,000 \\
\text { Norm: } 2,111,000\end{array}$ & $\begin{array}{l}\text { Period: } 1966-85 \\
\text { Max: } 9,799,000 \\
\text { Norm: } 3,530,000\end{array}$ & $\begin{array}{l}\text { Period: } 1986-97 \\
\text { Max: } 14,270,000 \\
\text { Norm: } 5,715,000\end{array}$ \\
\hline 41 & 08065500 & Trinity River near Midway, Texas & $31^{\circ} 04^{\prime} 28^{\prime \prime}$ & $95^{\circ} 41^{\prime} 57^{\prime \prime}$ & SE & 14,450 & 1940-70 & $\begin{array}{l}\text { Period: } 1940-53 \\
\text { Max: } 1,898,000 \\
\text { Norm: } 728,400\end{array}$ & $\begin{array}{l}\text { Period: } 1954-70 \\
\text { Max: } 6,907,000 \\
\text { Norm: } 2,127,000\end{array}$ & -- & -- \\
\hline 42 & 08066500 & Trinity River at Romayor, Texas & $30^{\circ} 25^{\prime} 30^{\prime \prime}$ & $94^{\circ} 51^{\prime} 02^{\prime \prime}$ & SE & 17,186 & 1924-97 & $\begin{array}{l}\text { Period: } 1924-53 \\
\text { Max: } 1,893,000 \\
\text { Norm: } 726,100\end{array}$ & $\begin{array}{l}\text { Period: } 1954-68 \\
\text { Max: } 7,310,000 \\
\text { Norm: } 2,205,000\end{array}$ & $\begin{array}{l}\text { Period: } 1969-85 \\
\text { Max: } 12,040,000 \\
\text { Norm: } 5,395,000\end{array}$ & $\begin{array}{l}\text { Period: } 1986-97 \\
\text { Max: } 16,440,000 \\
\text { Norm: } 7,568,000\end{array}$ \\
\hline 43 & 08067000 & Trinity River at Liberty, Texas & $30^{\circ} 03^{\prime} 27^{\prime \prime}$ & $94^{\circ} 49^{\prime} 05^{\prime \prime}$ & SE & 17,468 & 1940-97 & $\begin{array}{l}\text { Period: } 1940-53 \\
\text { Max: } 189,4000 \\
\text { Norm: } 726,700\end{array}$ & $\begin{array}{l}\text { Period: } 1954-68 \\
\text { Max: } 7,312,000 \\
\text { Norm: } 2,206,000\end{array}$ & $\begin{array}{l}\text { Period: } 1969-86 \\
\text { Max: } 12,040,000 \\
\text { Norm: } 5,398,000\end{array}$ & $\begin{array}{l}\text { Period: } 1987-97 \\
\text { Max: } 16,440,000 \\
\text { Norm: } 7,571,000\end{array}$ \\
\hline
\end{tabular}


Table 2. Static periods of regulation suitable for comparative analysis for 96 USGS streamflow-gaging stations with at least 10 years of annual peak-streamflow data through 1997 (designated as regulated by 10-percent criterion)—Continued

\begin{tabular}{|c|c|c|c|c|c|c|c|c|c|c|c|}
\hline $\begin{array}{l}\text { Seq. } \\
\text { no. }\end{array}$ & $\begin{array}{l}\text { USGS } \\
\text { station } \\
\text { number } \\
\text { (pl. 1) }\end{array}$ & USGS station name & Latitude & Longitude & Region & $\begin{array}{l}\text { Contri- } \\
\text { buting } \\
\text { drainage } \\
\text { area } \\
\left(\mathrm{mi}^{2}\right)\end{array}$ & $\begin{array}{l}\text { Total } \\
\text { period of } \\
\text { station } \\
\text { record } \\
\text { analyzed }\end{array}$ & $\begin{array}{c}\text { Static or } \\
\text { acceptably } \\
\text { static period } \\
\text { of regulation } \\
\text { no. } 1\end{array}$ & $\begin{array}{c}\text { Static or } \\
\text { acceptably } \\
\text { static period } \\
\text { of regulation } \\
\text { no. } 2\end{array}$ & $\begin{array}{c}\text { Static or } \\
\text { acceptably } \\
\text { static period } \\
\text { of regulation } \\
\text { no. } 3\end{array}$ & $\begin{array}{c}\text { Static or } \\
\text { acceptably } \\
\text { static period } \\
\text { of regulation } \\
\text { no. } 4\end{array}$ \\
\hline 44 & 08068000 & $\begin{array}{l}\text { West Fork San Jacinto River near Conroe, } \\
\text { Texas }\end{array}$ & $30^{\circ} 14^{\prime} 40^{\prime \prime}$ & $95^{\circ} 27^{\prime} 25^{\prime \prime}$ & SE & 828 & $1924-97$ & $\begin{array}{l}\text { Period: } 1924-72 \\
\text { Max: } 9,845 \\
\text { Norm: } 2,583\end{array}$ & $\begin{array}{l}\text { Period: } 1973-97 \\
\text { Max: 746,100 } \\
\text { Norm: 454,800 }\end{array}$ & -- & -- \\
\hline 45 & 08081000 & Salt Fork Brazos River near Peacock, Texas & $33^{\circ} 12^{\prime} 43^{\prime \prime}$ & $100^{\circ} 25^{\prime} 53^{\prime \prime}$ & $\mathrm{W}$ & 1,985 & $1950-86$ & $\begin{array}{l}\text { Period: } 1963-86 \\
\text { Max: } 137,100 \\
\text { Norm: } 52,790\end{array}$ & -- & -- & -- \\
\hline 46 & 08082000 & Salt Fork Brazos River near Aspermont, Texas & $33^{\circ} 20^{\prime} 02^{\prime \prime}$ & $100^{\circ} 14^{\prime} 16^{\prime \prime}$ & $\mathrm{W}$ & 2,496 & 1924-97 & $\begin{array}{l}\text { Period: } 1924-62 \\
\text { Max: } 1,421 \\
\text { Norm: } 358\end{array}$ & $\begin{array}{l}\text { Period: } 1963-97 \\
\text { Max: } 149,600 \\
\text { Norm: } 60,020\end{array}$ & -- & -- \\
\hline 47 & 08082500 & Brazos River at Seymour, Texas & $33^{\circ} 34^{\prime} 51^{\prime \prime}$ & $99^{\circ} 16^{\prime} 02^{\prime \prime}$ & $\mathrm{W}$ & 5,972 & 1924-97 & $\begin{array}{l}\text { Period: } 1924-62 \\
\text { Max: } 10,600 \\
\text { Norm: } 4,543\end{array}$ & $\begin{array}{l}\text { Period: } 1963-93 \\
\text { Max: } 247,000 \\
\text { Norm: } 88,590\end{array}$ & -- & -- \\
\hline 48 & 08087300 & Clear Fork Brazos River at Eliasville, Texas & $32^{\circ} 57^{\prime} 36^{\prime \prime}$ & $98^{\circ} 45^{\prime} 59^{\prime \prime}$ & $\mathrm{W}$ & 5,697 & $1916-82$ & $\begin{array}{l}\text { Period: } 1916-37 \\
\text { Max: } 105,500 \\
\text { Norm: } 109,800\end{array}$ & $\begin{array}{l}\text { Period: } 1938-61 \\
\text { Max: } 298,900 \\
\text { Norm: } 207,100\end{array}$ & $\begin{array}{l}\text { Period: } 1962-82 \\
\text { Max: } 1,185,000 \\
\text { Norm: } 589,200\end{array}$ & -- \\
\hline 49 & 08088000 & Brazos River near South Bend, Texas & $33^{\circ} 01^{\prime} 27^{\prime \prime}$ & $98^{\circ} 38^{\prime} 37^{\prime \prime}$ & $\mathrm{W}$ & 13,107 & 1939-93 & $\begin{array}{l}\text { Period: } 1939-73 \\
\text { Max: } 52,410 \\
\text { Norm: } 21,330\end{array}$ & $\begin{array}{l}\text { Period: } 1974-93 \\
\text { Max: } 413,100 \\
\text { Norm: } 142,500\end{array}$ & -- & -- \\
\hline 50 & 08090500 & Palo Pinto Creek near Santo, Texas & $32^{\circ} 37^{\prime} 51^{\prime \prime}$ & $98^{\circ} 10^{\prime} 50^{\prime \prime}$ & $\mathrm{NE}$ & 573 & $1951-76$ & $\begin{array}{l}\text { Period: } 1951-63 \\
\text { Max: } 7,242 \\
\text { Norm: } 3,675\end{array}$ & $\begin{array}{l}\text { Period: } 1964-76 \\
\text { Max: } 182,700 \\
\text { Norm: } 49,810\end{array}$ & -- & -- \\
\hline 51 & 08091000 & Brazos River near Glen Rose, Texas & $32^{\circ} 16^{\prime} 18^{\prime \prime}$ & $97^{\circ} 39^{\prime} 48^{\prime \prime}$ & $\mathrm{NE}$ & 16,252 & 1924-97 & $\begin{array}{l}\text { Period: } 1924-39 \\
\text { Max: } 172,600 \\
\text { Norm: } 137,300\end{array}$ & $\begin{array}{l}\text { Period: } 1940-61 \\
\text { Max: } 1,087,000 \\
\text { Norm: } 856,300\end{array}$ & $\begin{array}{l}\text { Period: } 1962-97 \\
\text { Max: } 2,632,000 \\
\text { Norm: } 1,531,000\end{array}$ & -- \\
\hline 52 & 08091500 & Paluxy River at Glen Rose, Texas & $32^{\circ} 13^{\prime} 53^{\prime \prime}$ & $97^{\circ} 46^{\prime} 37^{\prime \prime}$ & $\mathrm{NE}$ & 410 & $1948-97$ & $\begin{array}{l}\text { Period: } 1948-80 \\
\text { Max: } 2,819 \\
\text { Norm: } 1,653\end{array}$ & $\begin{array}{l}\text { Period: } 1981-97 \\
\text { Max: } 49,060 \\
\text { Norm: } 6,854\end{array}$ & -- & -- \\
\hline 53 & 08093500 & Aquilla Creek near Aquilla, Texas & $31^{\circ} 50^{\prime} 40^{\prime \prime}$ & $97^{\circ} 12^{\prime} 04^{\prime \prime}$ & $\mathrm{NE}$ & 308 & $1939-97$ & $\begin{array}{l}\text { Period: 1939-82 } \\
\text { Max: } 0 \\
\text { Norm: } 0\end{array}$ & $\begin{array}{l}\text { Period: } 1983-97 \\
\text { Max: } 374,100 \\
\text { Norm: 53,980 }\end{array}$ & -- & -- \\
\hline 54 & 08093700 & North Bosque River at Stephenville, Texas & $32^{\circ} 12^{\prime} 56^{\prime \prime}$ & $98^{\circ} 11^{\prime} 55^{\prime \prime}$ & $\mathrm{NE}$ & 95.9 & $1959-79$ & $\begin{array}{l}\text { Period: } 1959-79 \\
\text { Max: } 24,410 \\
\text { Norm: } 1,686\end{array}$ & -- & -- & -- \\
\hline
\end{tabular}


म $\quad$ Table 2. Static periods of regulation suitable for comparative analysis for 96 USGS streamflow-gaging stations with at least 10 years of annual peak-streamflow data through 1997 (designated as regulated by 10-percent criterion)—Continued

\begin{tabular}{|c|c|c|c|c|c|c|c|c|c|c|c|}
\hline $\begin{array}{l}\text { Seq. } \\
\text { no. }\end{array}$ & $\begin{array}{l}\text { USGS } \\
\text { station } \\
\text { number } \\
\text { (pl. 1) }\end{array}$ & USGS station name & Latitude & Longitude & Region & $\begin{array}{l}\text { Contri- } \\
\text { buting } \\
\text { drainage } \\
\text { area } \\
\left(\mathrm{mi}^{2}\right)\end{array}$ & $\begin{array}{l}\text { Total } \\
\text { period of } \\
\text { station } \\
\text { record } \\
\text { analyzed }\end{array}$ & $\begin{array}{c}\text { Static or } \\
\text { acceptably } \\
\text { static period } \\
\text { of regulation } \\
\text { no. } 1\end{array}$ & $\begin{array}{c}\text { Static or } \\
\text { acceptably } \\
\text { static period } \\
\text { of regulation } \\
\text { no. } 2\end{array}$ & $\begin{array}{c}\text { Static or } \\
\text { acceptably } \\
\text { static period } \\
\text { of regulation } \\
\text { no. } 3\end{array}$ & $\begin{array}{c}\text { Static or } \\
\text { acceptably } \\
\text { static period } \\
\text { of regulation } \\
\text { no. } 4\end{array}$ \\
\hline 55 & 08094800 & North Bosque River at Hico, Texas & $31^{\circ} 58^{\prime} 41^{\prime \prime}$ & $98^{\circ} 02^{\prime} 04^{\prime \prime}$ & NE & 359 & 1962-97 & $\begin{array}{l}\text { Period: } 1972-97 \\
\text { Max: 112,100 } \\
\text { Norm: 8,248 }\end{array}$ & -- & -- & -- \\
\hline 56 & 08095000 & North Bosque River near Clifton, Texas & $31^{\circ} 47^{\prime} 09^{\prime \prime}$ & $97^{\circ} 34^{\prime} 04^{\prime \prime}$ & $\mathrm{NE}$ & 968 & 1924-97 & $\begin{array}{l}\text { Period: } 1924-66 \\
\text { Max: } 14,410 \\
\text { Norm: } 4,564\end{array}$ & $\begin{array}{l}\text { Period: } 1967-97 \\
\text { Max: } 134,100 \\
\text { Norm: } 14,580\end{array}$ & -- & -- \\
\hline 57 & 08095400 & Hog Creek near Crawford, Texas & $31^{\circ} 33^{\prime} 20^{\prime \prime}$ & $97^{\circ} 21^{\prime 22 "}$ & $\mathrm{NE}$ & 78.2 & 1960-97 & $\begin{array}{l}\text { Period: } 1960-76 \\
\text { Max: } 0 \\
\text { Norm: } 0\end{array}$ & $\begin{array}{l}\text { Period: } 1977-97 \\
\text { Max: } 17,280 \\
\text { Norm: } 936\end{array}$ & -- & -- \\
\hline 58 & 08096500 & Brazos River at Waco, Texas & $31^{\circ} 32^{\prime} 06^{\prime \prime}$ & $97^{\circ} 04^{\prime} 22^{\prime \prime}$ & $\mathrm{NE}$ & 20,007 & 1899-1997 & $\begin{array}{l}\text { Period: } 1899-1949 \\
\text { Max: } 172,600 \\
\text { Norm: } 138,300\end{array}$ & $\begin{array}{l}\text { Period: } 1950-70 \\
\text { Max: } 3,700,000 \\
\text { Norm: 1,701,000 }\end{array}$ & $\begin{array}{l}\text { Period: } 1971-97 \\
\text { Max: 5,501,000 } \\
\text { Norm: 2,424,000 }\end{array}$ & -- \\
\hline 59 & 08099500 & Leon River near Hasse, Texas & $31^{\circ} 57^{\prime} 28^{\prime \prime}$ & $98^{\circ} 27^{\prime} 32^{\prime \prime}$ & $\mathrm{W}$ & 1,261 & 1939-91 & $\begin{array}{l}\text { Period: } 1939-62 \\
\text { Max: 30,330 } \\
\text { Norm: 4,021 }\end{array}$ & $\begin{array}{l}\text { Period: } 1963-91 \\
\text { Max: } 580,000 \\
\text { Norm: } 102,400\end{array}$ & -- & -- \\
\hline 60 & 08100000 & Leon River near Hamilton, Texas & $31^{\circ} 47^{\prime} 19^{\prime \prime}$ & $98^{\circ} 07^{\prime} 16^{\prime \prime}$ & $\mathrm{NE}$ & 1,891 & $1925-97$ & $\begin{array}{l}\text { Period: } 1963-97 \\
\text { Max: } 657,600 \\
\text { Norm: } 113,400\end{array}$ & -- & -- & -- \\
\hline 61 & 08102500 & Leon River near Belton, Texas & $31^{\circ} 04^{\prime} 12^{\prime \prime}$ & $97^{\circ} 26^{\prime} 28^{\prime \prime}$ & NE & 3,542 & 1924-97 & $\begin{array}{l}\text { Period: } 1924-53 \\
\text { Max: } 35,690 \\
\text { Norm: 6,597 }\end{array}$ & $\begin{array}{l}\text { Period: } 1954-97 \\
\text { Max: 2,572,000 } \\
\text { Norm: 577,100 }\end{array}$ & -- & -- \\
\hline 62 & 08104700 & $\begin{array}{l}\text { North Fork San Gabriel River near } \\
\text { Georgetown, Texas }\end{array}$ & $30^{\circ} 39^{\prime} 42^{\prime \prime}$ & $97^{\circ} 42^{\prime} 40^{\prime \prime}$ & $\mathrm{W}$ & 248 & 1969-97 & $\begin{array}{l}\text { Period: } 1969-79 \\
\text { Max: } 72 \\
\text { Norm: } 26\end{array}$ & $\begin{array}{l}\text { Period: } 1980-97 \\
\text { Max: } 220,200 \\
\text { Norm: } 37,130\end{array}$ & -- & -- \\
\hline 63 & 08105700 & San Gabriel River at Laneport, Texas & $30^{\circ} 41^{\prime} 39^{\prime \prime}$ & $97^{\circ} 16^{\prime} 43^{\prime \prime}$ & $\mathrm{W}$ & 738 & $1965-97$ & $\begin{array}{l}\text { Period: } 1965-78 \\
\text { Max: } 8,053 \\
\text { Norm: } 723\end{array}$ & $\begin{array}{l}\text { Period: } 1979-97 \\
\text { Max: 789,600 } \\
\text { Norm: } 103,500\end{array}$ & -- & -- \\
\hline 64 & 08106500 & Little River at Cameron, Texas & $30^{\circ} 49^{\prime} 53^{\prime \prime}$ & $96^{\circ} 57^{\prime} 01^{\prime \prime}$ & SE & 7,065 & 1918-97 & $\begin{array}{l}\text { Period: } 1918-53 \\
\text { Max: } 35,970 \\
\text { Norm: } 6,687\end{array}$ & $\begin{array}{l}\text { Period: } 1954-67 \\
\text { Max: } 2,308,000 \\
\text { Norm: } 535,800\end{array}$ & $\begin{array}{l}\text { Period: } 1968-97 \\
\text { Max: 4,538,000 } \\
\text { Norm: } 930,500\end{array}$ & -- \\
\hline 65 & 08109000 & Brazos River near Bryan, Texas & $30^{\circ} 36^{\prime} 52^{\prime \prime}$ & $96^{\circ} 29^{\prime} 10^{\prime \prime}$ & SE & 29,949 & $1900-95$ & $\begin{array}{l}\text { Period: } 1900-50 \\
\text { Max: } 218,100 \\
\text { Norm: } 151,600\end{array}$ & $\begin{array}{l}\text { Period: } 1951-64 \\
\text { Max: } 5,390,000 \\
\text { Norm: } 2,054,000\end{array}$ & $\begin{array}{l}\text { Period: } 1965-95 \\
\text { Max: } 9,970,000 \\
\text { Norm: } 3,414,000\end{array}$ & -- \\
\hline
\end{tabular}


Table 2. Static periods of regulation suitable for comparative analysis for 96 USGS streamflow-gaging stations with at least 10 years of annual peak-streamflow data through 1997 (designated as regulated by 10-percent criterion)—Continued

\begin{tabular}{|c|c|c|c|c|c|c|c|c|c|c|c|}
\hline $\begin{array}{c}\text { Seq. } \\
\text { no. }\end{array}$ & $\begin{array}{l}\text { USGS } \\
\text { station } \\
\text { number } \\
\text { (pl. 1) }\end{array}$ & USGS station name & Latitude & Longitude & Region & $\begin{array}{l}\text { Contri- } \\
\text { buting } \\
\text { drainage } \\
\text { area } \\
\left(\mathrm{mi}^{2}\right)\end{array}$ & $\begin{array}{l}\text { Total } \\
\text { period of } \\
\text { station } \\
\text { record } \\
\text { analyzed }\end{array}$ & $\begin{array}{c}\text { Static or } \\
\text { acceptably } \\
\text { static period } \\
\text { of regulation } \\
\text { no. } 1\end{array}$ & $\begin{array}{c}\text { Static or } \\
\text { acceptably } \\
\text { static period } \\
\text { of regulation } \\
\text { no. } 2\end{array}$ & $\begin{array}{c}\text { Static or } \\
\text { acceptably } \\
\text { static period } \\
\text { of regulation } \\
\text { no. } 3\end{array}$ & $\begin{array}{c}\text { Static or } \\
\text { acceptably } \\
\text { static period } \\
\text { of regulation } \\
\text { no. } 4\end{array}$ \\
\hline 66 & 08110000 & Yegua Creek near Somerville, Texas & $30^{\circ} 19^{\prime} 18^{\prime \prime}$ & $96^{\circ} 30^{\prime} 26^{\prime \prime}$ & $\mathrm{SE}$ & 1,009 & 1925-91 & $\begin{array}{l}\text { Period: } 1925-66 \\
\text { Max: } 10,000 \\
\text { Norm: } 5,000\end{array}$ & $\begin{array}{l}\text { Period: } 1967-91 \\
\text { Max: } 1,055,000 \\
\text { Norm: } 179,100\end{array}$ & -- & -- \\
\hline 67 & 08111000 & Navasota River near Bryan, Texas & $30^{\circ} 52^{\prime} 10^{\prime \prime}$ & $96^{\circ} 11^{\prime} 32^{\prime \prime}$ & SE & 1,454 & 1951-97 & $\begin{array}{l}\text { Period: } 1951-77 \\
\text { Max: } 50,550 \\
\text { Norm: } 13,250\end{array}$ & $\begin{array}{l}\text { Period: } 1978-97 \\
\text { Max: } 513,300 \\
\text { Norm: } 276,600\end{array}$ & -- & -- \\
\hline 68 & 08111500 & Brazos River near Hempstead, Texas & $30^{\circ} 07^{\prime} 44^{\prime \prime}$ & $96^{\circ} 11^{\prime} 15^{\prime \prime}$ & SE & 34,314 & 1939-97 & $\begin{array}{l}\text { Period: } 1939-50 \\
\text { Max: } 956,000 \\
\text { Norm: } 798,300\end{array}$ & $\begin{array}{l}\text { Period: } 1951-66 \\
\text { Max: } 6,370,000 \\
\text { Norm: } 2,444,000\end{array}$ & $\begin{array}{l}\text { Period: } 1967-97 \\
\text { Max: } 12,100,000 \\
\text { Norm: } 3,936,000\end{array}$ & -- \\
\hline 69 & 08114000 & Brazos River at Richmond, Texas & $29^{\circ} 34^{\prime} 56^{\prime \prime}$ & $95^{\circ} 45^{\prime} 27^{\prime \prime}$ & SE & 35,441 & 1903-97 & $\begin{array}{l}\text { Period: } 1903-50 \\
\text { Max: } 355,100 \\
\text { Norm: } 229,600\end{array}$ & $\begin{array}{l}\text { Period: } 1951-67 \\
\text { Max: } 6,371,000 \\
\text { Norm: } 2,445,000\end{array}$ & $\begin{array}{l}\text { Period: } 1968-97 \\
\text { Max: } 12,100,000 \\
\text { Norm: } 3,938,000\end{array}$ & -- \\
\hline 70 & 08121000 & Colorado River at Colorado City, Texas & $32^{\circ} 23^{\prime} 33^{\prime \prime}$ & $100^{\circ} 52^{\prime} 42^{\prime \prime}$ & $\mathrm{w}$ & 1,585 & 1947-97 & $\begin{array}{l}\text { Period: } 1952-97 \\
\text { Max: } 363,210 \\
\text { Norm: } 205,600\end{array}$ & -- & -- & -- \\
\hline 71 & 08124000 & Colorado River at Robert Lee, Texas & $31^{\circ} 53^{\prime} 07^{\prime \prime}$ & $100^{\circ} 28^{\prime} 49^{\prime \prime}$ & $\mathrm{W}$ & 5,047 & 1924-97 & $\begin{array}{l}\text { Period: } 1924-51 \\
\text { Max: } 10,380 \\
\text { Norm: } 6,446\end{array}$ & $\begin{array}{l}\text { Period: } 1951-68 \\
\text { Max: 535,200 } \\
\text { Norm: 286,500 }\end{array}$ & $\begin{array}{l}\text { Period: } 1969-88 \\
\text { Max: } 1,349,000 \\
\text { Norm: } 777,300\end{array}$ & $\begin{array}{l}\text { Period: } 1989-97 \\
\text { Max: 1,627,000 } \\
\text { Norm: } 880,400\end{array}$ \\
\hline 72 & 08135000 & North Concho River at San Angelo, Texas & $31^{\circ} 27^{\prime} 57^{\prime \prime}$ & $100^{\circ} 26^{\prime} 51^{\prime \prime}$ & $\mathrm{w}$ & 1,450 & $1916-90$ & $\begin{array}{l}\text { Period: } 1916-51 \\
\text { Max: } 80 \\
\text { Norm: } 80\end{array}$ & $\begin{array}{l}\text { Period: } 1952-90 \\
\text { Max: } 696,600 \\
\text { Norm: } 119,300\end{array}$ & -- & -- \\
\hline 73 & 08136000 & Concho River at San Angelo, Texas & $31^{\circ} 27^{\prime} 16^{\prime \prime}$ & $100^{\circ} 24^{\prime} 37^{\prime \prime}$ & $\mathrm{W}$ & 4,411 & $1916-97$ & $\begin{array}{l}\text { Period: } 1916-51 \\
\text { Max: 44,140 } \\
\text { Norm: } 15,530\end{array}$ & $\begin{array}{l}\text { Period: } 1952-62 \\
\text { Max: } 741,100 \\
\text { Norm: } 135,100\end{array}$ & $\begin{array}{l}\text { Period: } 1963-97 \\
\text { Max: 1,829,000 } \\
\text { Norm: } 321,300\end{array}$ & -- \\
\hline 74 & 08136500 & Concho River at Paint Rock, Texas & $31^{\circ} 30^{\prime} 57^{\prime \prime}$ & $99^{\circ} 55^{\prime} 09^{\prime \prime}$ & $\mathrm{w}$ & 5,443 & 1916-97 & $\begin{array}{l}\text { Period: } 1916-51 \\
\text { Max: } 45,050 \\
\text { Norm: } 16,340\end{array}$ & $\begin{array}{l}\text { Period: } 1952-62 \\
\text { Max: } 742,700 \\
\text { Norm: } 136,300\end{array}$ & $\begin{array}{l}\text { Period: } 1963-97 \\
\text { Max: 1,831,000 } \\
\text { Norm: } 322,800\end{array}$ & -- \\
\hline 75 & 08137500 & Mukewater Creek at Trickham, Texas & $31^{\circ} 35^{\prime} 24^{\prime \prime}$ & $99^{\circ} 13^{\prime} 36^{\prime \prime}$ & $\mathrm{W}$ & 70.0 & $1951-73$ & $\begin{array}{l}\text { Period: } 1960-73 \\
\text { Max: } 17,120 \\
\text { Norm: } 1,052\end{array}$ & -- & -- & -- \\
\hline 76 & 08138000 & Colorado River at Winchell, Texas & $31^{\circ} 28^{\prime} 04^{\prime \prime}$ & $99^{\circ} 09^{\prime} 43^{\prime \prime}$ & $\mathrm{w}$ & 13,788 & 1924-97 & $\begin{array}{l}\text { Period: } 1924-50 \\
\text { Max: } 65,730 \\
\text { Norm: } 24,760\end{array}$ & $\begin{array}{l}\text { Period: } 1951-62 \\
\text { Max: } 1,387,000 \\
\text { Norm: } 468,200\end{array}$ & $\begin{array}{l}\text { Period: } 1970-97 \\
\text { Max: } 3,560,000 \\
\text { Norm: } 1,182,000\end{array}$ & -- \\
\hline
\end{tabular}


ळ Table 2. Static periods of regulation suitable for comparative analysis for 96 USGS streamflow-gaging stations with at least 10 years of annual peak-streamflow data through 1997 (designated as regulated by 10-percent criterion)-Continued

\begin{tabular}{|c|c|c|c|c|c|c|c|c|c|c|c|}
\hline $\begin{array}{l}\text { Seq. } \\
\text { no. }\end{array}$ & $\begin{array}{l}\text { USGS } \\
\text { station } \\
\text { number } \\
\text { (pl. 1) }\end{array}$ & USGS station name & Latitude & Longitude & Region & $\begin{array}{l}\text { Contri- } \\
\text { buting } \\
\text { drainage } \\
\text { area } \\
\left(\mathrm{mi}^{2}\right)\end{array}$ & $\begin{array}{l}\text { Total } \\
\text { period of } \\
\text { station } \\
\text { record } \\
\text { analyzed }\end{array}$ & $\begin{array}{c}\text { Static or } \\
\text { acceptably } \\
\text { static period } \\
\text { of regulation } \\
\text { no. } 1\end{array}$ & $\begin{array}{c}\text { Static or } \\
\text { acceptably } \\
\text { static period } \\
\text { of regulation } \\
\text { no. } 2\end{array}$ & $\begin{array}{c}\text { Static or } \\
\text { acceptably } \\
\text { static period } \\
\text { of regulation } \\
\text { no. } 3\end{array}$ & $\begin{array}{c}\text { Static or } \\
\text { acceptably } \\
\text { static period } \\
\text { of regulation } \\
\text { no. } 4\end{array}$ \\
\hline 77 & 08143500 & Pecan Bayou at Brownwood, Texas & $31^{\circ} 43^{\prime} 54^{\prime \prime}$ & $98^{\circ} 58^{\prime} 25^{\prime \prime}$ & $\mathrm{W}$ & 1,660 & $1924-83$ & $\begin{array}{l}\text { Period: } 1933-62 \\
\text { Max: } 457,400 \\
\text { Norm: } 123,300\end{array}$ & $\begin{array}{l}\text { Period: } 1966-83 \\
\text { Max: } 791,400 \\
\text { Norm: } 185,200\end{array}$ & -- & -- \\
\hline 78 & 08145000 & Brady Creek at Brady, Texas & $31^{\circ} 08^{\prime} 17^{\prime \prime}$ & $99^{\circ} 20^{\prime} 05^{\prime \prime}$ & $\mathrm{W}$ & 588 & $1940-85$ & $\begin{array}{l}\text { Period: } 1940-62 \\
\text { Max: } 31,940 \\
\text { Norm: } 1,288\end{array}$ & $\begin{array}{l}\text { Period: } 1963-85 \\
\text { Max: } 355,400 \\
\text { Norm: } 35,210\end{array}$ & -- & -- \\
\hline 79 & 08146000 & San Saba River at San Saba, Texas & $31^{\circ} 12^{\prime} 47^{\prime \prime}$ & $98^{\circ} 43^{\prime} 09^{\prime \prime}$ & $\mathrm{W}$ & 3,039 & 1916-94 & $\begin{array}{l}\text { Period: } 1916-62 \\
\text { Max: } 1,146 \\
\text { Norm: } 733\end{array}$ & $\begin{array}{l}\text { Period: } 1963-94 \\
\text { Max: } 392,600 \\
\text { Norm: } 38,470\end{array}$ & -- & -- \\
\hline 80 & 08147000 & Colorado River near San Saba, Texas & $31^{\circ} 13^{\prime} 04^{\prime \prime}$ & $98^{\circ} 33^{\prime} 51^{\prime \prime}$ & $\mathrm{W}$ & 19,819 & 1916-97 & $\begin{array}{l}\text { Period: } 1916-51 \\
\text { Max: } 290,800 \\
\text { Norm: } 84,080\end{array}$ & $\begin{array}{l}\text { Period: } 1952-62 \\
\text { Max: 1,880,000 } \\
\text { Norm: 563,400 }\end{array}$ & $\begin{array}{l}\text { Period: } 1969-97 \\
\text { Max: 4,902,000 } \\
\text { Norm: } 1,421,000\end{array}$ & -- \\
\hline 81 & 08158000 & Colorado River at Austin, Texas & $30^{\circ} 14^{\prime} 40^{\prime \prime}$ & $97^{\circ} 41^{\prime} 39^{\prime \prime}$ & $\mathrm{W}$ & 27,606 & 1899-1997 & $\begin{array}{l}\text { Period: } 1899-1941 \\
\text { Max: } 69,000 \\
\text { Norm: } 25,300\end{array}$ & $\begin{array}{l}\text { Period: } 1942-62 \\
\text { Max: 5,866,000 } \\
\text { Norm: } 2,604,000\end{array}$ & $\begin{array}{l}\text { Period: } 1963-97 \\
\text { Max: 9,428,000 } \\
\text { Norm: 3,611,000 }\end{array}$ & -- \\
\hline 82 & 08159500 & Colorado River at Smithville, Texas & $30^{\circ} 00^{\prime} 43^{\prime \prime}$ & $97^{\circ} 09^{\prime} 43^{\prime \prime}$ & $\mathrm{SE}$ & 29,058 & $1931-97$ & $\begin{array}{l}\text { Period: } 1931-41 \\
\text { Max: } 518,400 \\
\text { Norm: } 144,900\end{array}$ & $\begin{array}{l}\text { Period: } 1942-62 \\
\text { Max: 5,867,000 } \\
\text { Norm: } 2,605,000\end{array}$ & $\begin{array}{l}\text { Period: } 1963-97 \\
\text { Max: 9,497,000 } \\
\text { Norm: 3,664,000 }\end{array}$ & -- \\
\hline 83 & 08161000 & Colorado River at Columbus, Texas & $29^{\circ} 42^{\prime} 22^{\prime \prime}$ & $96^{\circ} 32^{\prime} 12^{\prime \prime}$ & SE & 30,237 & $1916-97$ & $\begin{array}{l}\text { Period: } 1916-41 \\
\text { Max: } 69,330 \\
\text { Norm: } 25,480\end{array}$ & $\begin{array}{l}\text { Period: } 1942-62 \\
\text { Max: 5,868,000 } \\
\text { Norm: } 2,605,000\end{array}$ & $\begin{array}{l}\text { Period: } 1963-97 \\
\text { Max: } 9,643,000 \\
\text { Norm: } 3,744,000\end{array}$ & -- \\
\hline 84 & 08168500 & $\begin{array}{l}\text { Guadalupe River above Comal River at } \\
\text { New Braunfels, Texas }\end{array}$ & $29^{\circ} 42^{\prime} 53^{\prime \prime}$ & $98^{\circ} 06^{\prime} 35^{\prime \prime}$ & $\mathrm{W}$ & 1,518 & $1928-97$ & $\begin{array}{l}\text { Period: } 1928-63 \\
\text { Max: } 277 \\
\text { Norm: } 184\end{array}$ & $\begin{array}{l}\text { Period: } 1964-97 \\
\text { Max: 1,146,000 } \\
\text { Norm: } 392,200\end{array}$ & -- & -- \\
\hline 85 & 08169500 & Guadalupe River at New Braunfels, Texas & $29^{\circ} 41^{\prime} 52^{\prime \prime}$ & $98^{\circ} 06^{\prime} 23^{\prime \prime}$ & $\mathrm{W}$ & 1,652 & $1915-97$ & $\begin{array}{l}\text { Period: } 1915-27 \\
\text { Max: } 74 \\
\text { Norm: } 74\end{array}$ & $\begin{array}{l}\text { Period: } 1974-97 \\
\text { Max: 1,146,000 } \\
\text { Norm: } 392,300\end{array}$ & -- & -- \\
\hline 86 & 08172000 & San Marcos River at Luling, Texas & $29^{\circ} 39^{\prime} 54^{\prime \prime}$ & $97^{\circ} 38^{\prime} 59^{\prime \prime}$ & $\mathrm{SE}$ & 838 & 1940-97 & $\begin{array}{l}\text { Period: } 1940-82 \\
\text { Max: } 24,260 \\
\text { Norm: } 4,490\end{array}$ & $\begin{array}{l}\text { Period: } 1983-97 \\
\text { Max: } 74,830 \\
\text { Norm: } 7,880\end{array}$ & -- & -- \\
\hline 87 & 08173000 & Plum Creek near Luling, Texas & $29^{\circ} 41^{\prime} 58^{\prime \prime}$ & $97^{\circ} 36^{\prime} 12^{\prime \prime}$ & SE & 309 & $1930-93$ & $\begin{array}{l}\text { Period: } 1930-66 \\
\text { Max: } 0 \\
\text { Norm: } 0\end{array}$ & $\begin{array}{l}\text { Period: } 1967-93 \\
\text { Max: } 59,110 \\
\text { Norm: } 3,921\end{array}$ & -- & -- \\
\hline
\end{tabular}


Table 2. Static periods of regulation suitable for comparative analysis for 96 USGS streamflow-gaging stations with at least 10 years of annual peak-streamflow data through 1997 (designated as regulated by 10-percent criterion)—Continued

\begin{tabular}{|c|c|c|c|c|c|c|c|c|c|c|c|}
\hline $\begin{array}{c}\text { Seq. } \\
\text { no. }\end{array}$ & $\begin{array}{c}\text { USGS } \\
\text { station } \\
\text { number } \\
\text { (pl. 1) }\end{array}$ & USGS station name & Latitude & Longitude & Region & $\begin{array}{l}\text { Contri- } \\
\text { buting } \\
\text { drainage } \\
\text { area } \\
\left(\mathrm{mi}^{2}\right)\end{array}$ & $\begin{array}{l}\text { Total } \\
\text { period of } \\
\text { station } \\
\text { record } \\
\text { analyzed }\end{array}$ & $\begin{array}{c}\text { Static or } \\
\text { acceptably } \\
\text { static period } \\
\text { of regulation } \\
\text { no. } 1\end{array}$ & $\begin{array}{c}\text { Static or } \\
\text { acceptably } \\
\text { static period } \\
\text { of regulation } \\
\text { no. } 2\end{array}$ & $\begin{array}{c}\text { Static or } \\
\text { acceptably } \\
\text { static period } \\
\text { of regulation } \\
\text { no. } 3\end{array}$ & $\begin{array}{c}\text { Static or } \\
\text { acceptably } \\
\text { static period } \\
\text { of regulation } \\
\text { no. } 4\end{array}$ \\
\hline 88 & 08176500 & Guadalupe River at Victoria, Texas & $28^{\circ} 47^{\prime} 34^{\prime \prime}$ & $97^{\circ} 00^{\prime} 46^{\prime \prime}$ & $\mathrm{SE}$ & 5,198 & 1935-97 & $\begin{array}{l}\text { Period: } 1935-63 \\
\text { Max: } 49,710 \\
\text { Norm: } 24,620\end{array}$ & $\begin{array}{l}\text { Period: } 1964-97 \\
\text { Max: } 1,353,000 \\
\text { Norm: } 434,500\end{array}$ & -- & -- \\
\hline 89 & 08177500 & Coleto Creek near Victoria, Texas & $28^{\circ} 43^{\prime} 51^{\prime \prime}$ & $97^{\circ} 08^{\prime} 18^{\prime \prime}$ & SE & 514 & 1939-97 & $\begin{array}{l}\text { Period: } 1939-54 \\
\text { Max: } 0 \\
\text { Norm: } 0\end{array}$ & $\begin{array}{l}\text { Period: } 1980-97 \\
\text { Max: } 169,100 \\
\text { Norm: } 35,160\end{array}$ & -- & -- \\
\hline 90 & 08183500 & San Antonio River near Falls City, Texas & $28^{\circ} 57^{\prime} 05^{\prime \prime}$ & $98^{\circ} 03^{\prime} 50^{\prime \prime}$ & $\mathrm{SE}$ & 2,113 & 1926-97 & $\begin{array}{l}\text { Period: } 1926-68 \\
\text { Max: } 356,900 \\
\text { Norm: } 261,500\end{array}$ & $\begin{array}{l}\text { Period: } 1969-97 \\
\text { Max: } 601,500 \\
\text { Norm: } 361,000\end{array}$ & -- & -- \\
\hline 91 & 08183900 & Cibolo Creek near Boerne, Texas & $29^{\circ} 46^{\prime} 26^{\prime \prime}$ & $98^{\circ} 41^{\prime} 50^{\prime \prime}$ & $\mathrm{w}$ & 68.4 & 1963-94 & $\begin{array}{l}\text { Period: } 1963-77 \\
\text { Max: } 575 \\
\text { Norm: } 332\end{array}$ & $\begin{array}{l}\text { Period: } 1978-94 \\
\text { Max: } 23,330 \\
\text { Norm: } 4,850\end{array}$ & -- & -- \\
\hline 92 & 08185000 & Cibolo Creek at Selma, Texas & $29^{\circ} 35^{\prime} 38^{\prime \prime}$ & $98^{\circ} 18^{\prime} 39^{\prime \prime}$ & $\mathrm{w}$ & 274 & 1946-97 & $\begin{array}{l}\text { Period: } 1946-77 \\
\text { Max: } 575 \\
\text { Norm: } 332\end{array}$ & $\begin{array}{l}\text { Period: } 1978-97 \\
\text { Max: } 23,330 \\
\text { Norm: } 4,850\end{array}$ & -- & -- \\
\hline 93 & 08188500 & San Antonio River at Goliad, Texas & $28^{\circ} 38^{\prime} 58^{\prime \prime}$ & $97^{\circ} 23^{\prime} 04^{\prime \prime}$ & SE & 3,921 & 1924-97 & $\begin{array}{l}\text { Period: } 1924-68 \\
\text { Max: } 375,500 \\
\text { Norm: } 264,200\end{array}$ & $\begin{array}{l}\text { Period: } 1969-97 \\
\text { Max: } 673,500 \\
\text { Norm: } 375,100\end{array}$ & -- & -- \\
\hline 94 & 08193000 & Nueces River near Asherton, Texas & $28^{\circ} 30^{\prime} 00^{\prime \prime}$ & $99^{\circ} 40^{\prime} 54^{\prime \prime}$ & $\mathrm{W}$ & 4,082 & 1940-97 & $\begin{array}{l}\text { Period: } 1940-62 \\
\text { Max: } 22,020 \\
\text { Norm: } 10,030\end{array}$ & $\begin{array}{l}\text { Period: } 1963-97 \\
\text { Max: } 43,070 \\
\text { Norm: } 19,500\end{array}$ & -- & -- \\
\hline 95 & 08194000 & Nueces River at Cotulla, Texas & $28^{\circ} 25^{\prime} 34^{\prime \prime}$ & $99^{\circ} 14^{\prime} 23^{\prime \prime}$ & $\mathrm{W}$ & 5,171 & 1924-97 & $\begin{array}{l}\text { Period: } 1924-46 \\
\text { Max: 8,211 } \\
\text { Norm: 5,343 }\end{array}$ & $\begin{array}{l}\text { Period: } 1947-62 \\
\text { Max: } 35,280 \\
\text { Norm: } 15,210\end{array}$ & $\begin{array}{l}\text { Period: } 1963-97 \\
\text { Max: } 61,870 \\
\text { Norm: } 27,730\end{array}$ & -- \\
\hline 96 & 08194500 & Nueces River near Tilden, Texas & $28^{\circ} 18^{\prime} 31^{\prime \prime}$ & $98^{\circ} 33^{\prime} 25^{\prime \prime}$ & $\mathrm{w}$ & 8,093 & 1942-97 & $\begin{array}{l}\text { Period: } 1947-62 \\
\text { Max: } 47,940 \\
\text { Norm: } 20,890\end{array}$ & $\begin{array}{l}\text { Period: } 1963-97 \\
\text { Max: } 98,710 \\
\text { Norm: } 43,390\end{array}$ & -- & -- \\
\hline
\end{tabular}


Table 3. Texas Natural Resource Conservation Commission permitted dams with maximum capacities in excess

Type of spillway: $\quad$ C, controlled; U, uncontrolled

Purpose of dam: $\quad$ FC, flood control; O, other

Owner name: $\quad$ WID, Water Improvement District; WCID, Water Control and Improvement District; MUD, FWSD, Fresh Water Supply District; WSD, Water Supply District; MWSD, Municipal Water Supply Updated Information: max. dis., maximum discharge; DA, drainage area; SA, surface area; ph. phone; dis. freq., discharge [See text for specific definition of some columns. Seq., sequence; TNRCC, Texas Natural Resource Conservation recurrence interval; PMF, probable maximum flood; SCS, Soil Conservation Service]

\begin{tabular}{|c|c|c|c|c|c|c|c|c|c|}
\hline $\begin{array}{l}\text { Seq. } \\
\text { no. }\end{array}$ & $\begin{array}{c}\text { TNRCC } \\
\text { no. } \\
\text { (pl. 2) }\end{array}$ & $\begin{array}{l}\text { River } \\
\text { basin }\end{array}$ & $\begin{array}{c}\text { Stream } \\
\text { name }\end{array}$ & County & $\begin{array}{c}\text { Reservoir } \\
\text { name }\end{array}$ & Latitude & Longitude & $\begin{array}{c}\text { Year } \\
\text { com- } \\
\text { pleted }\end{array}$ & $\begin{array}{c}\text { Drain- } \\
\text { age } \\
\text { area } \\
\left(\mathrm{mi}^{2}\right)\end{array}$ \\
\hline 1 & TX00018 & San Jacinto & South Mayde Creek & Harris & Addicks Reservoir & $29^{\circ} 47^{\prime} 30$ & $95^{\circ} 37^{\prime} 24^{\prime \prime}$ & 1948 & -- \\
\hline 2 & TX02376 & Brazos & Sandy Creek & Milam & Alcoa Lake & $30^{\circ} 34^{\prime} 30^{\prime \prime}$ & $97^{\circ} 02^{\prime} 54^{\prime \prime}$ & 1952 & -- \\
\hline 3 & TX02296 & Rio Grande & Rio Grande & Val Verde & Amistad Reservoir & $29^{\circ} 27^{\prime} 00^{\prime \prime}$ & $101^{\circ} 03^{\prime} 30^{\prime \prime}$ & 1969 & 123,133 \\
\hline
\end{tabular}

\begin{tabular}{|c|c|c|c|c|c|c|c|c|c|}
\hline 4 & TX08004 & Brazos & Aquilla Creek & Hill & Aquilla Lake & $31^{\circ} 54^{\prime} 48^{\prime \prime}$ & $97^{\circ} 12^{\prime} 30^{\prime \prime}$ & 1983 & -- \\
\hline 5 & TX00015 & Neches & Neches River & Tyler & B.A. Steinhagen Lake & $30^{\circ} 47^{\prime} 00^{\prime \prime}$ & $94^{\circ} 10^{\prime} 00^{\prime \prime}$ & 1951 & -- \\
\hline 6 & TX05952 & Colorado & Valley Creek & Runnels & Ballinger Municipal Lake & $31^{\circ} 43^{\prime} 48^{\prime \prime}$ & $100^{\circ} 02^{\prime} 36^{\prime \prime}$ & 1985 & -- \\
\hline 7 & TX00001 & Trinity & Waxahachie Creek & Ellis & Bardwell Lake & $32^{\circ} 16^{\prime} 00^{\prime \prime}$ & $96^{\circ} 38^{\prime} 00^{\prime \prime}$ & 1965 & -- \\
\hline 8 & TX00019 & San Jacinto & Buffalo Bayou & Harris & Barker Reservoir & $29^{\circ} 46^{\prime} 12^{\prime \prime}$ & $95^{\circ} 38^{\prime} 48^{\prime \prime}$ & 1945 & -- \\
\hline 9 & TX03622 & Red & Baylor Creek & Childress & Baylor Lake & $34^{\circ} 28^{\prime} 36^{\prime \prime}$ & $100^{\circ} 22^{\prime} 18^{\prime \prime}$ & 1950 & 34.2 \\
\hline 10 & TX00002 & Brazos & Leon River & Bell & Belton Lake & $31^{\circ} 06^{\prime} 00^{\prime \prime}$ & $97^{\circ} 29^{\prime} 00^{\prime \prime}$ & 1954 & -- \\
\hline 11 & TX00003 & Trinity & Clear Fork Trinity River & Tarrant & Benbrook Lake & $32^{\circ} 39^{\prime} 00^{\prime \prime}$ & $97^{\circ} 27^{\prime} 00^{\prime \prime}$ & 1950 & -- \\
\hline 12 & TX01659 & Colorado & Brady Creek & McCulloch & Brady Reservoir & $31^{\circ} 08^{\prime} 24^{\prime \prime}$ & $99^{\circ} 23^{\prime} 30^{\prime \prime}$ & 1963 & -- \\
\hline 13 & TX04833 & Sabine & Brandy Branch & Harrison & Brandy Branch Cooling Pond & $32^{\circ} 25^{\prime} 48^{\prime \prime}$ & $94^{\circ} 29^{\prime} 06^{\prime \prime}$ & 1983 & 4.1 \\
\hline 14 & TX01432 & San Antonio & Arroyo Seco & Bexar & Braunig Lake & $29^{\circ} 14^{\prime} 24^{\prime \prime}$ & $98^{\circ} 22^{\prime} 18^{\prime \prime}$ & 1963 & 9.4 \\
\hline 15 & TX02789 & Colorado & Pecan Bayou & Brown & Brownwood Reservoir & $31^{\circ} 50^{\prime} 18^{\prime \prime}$ & $99^{\circ} 00^{\prime} 06^{\prime \prime}$ & 1933 & 2.4 \\
\hline 16 & TX01869 & Brazos & $\begin{array}{l}\text { Trib.-Thompsons and Peak } \\
\text { Creeks }\end{array}$ & Brazos & Bryan Utilities Lake & $30^{\circ} 42^{\prime} 30^{\prime \prime}$ & $96^{\circ} 27^{\prime} 06^{\prime \prime}$ & 1975 & -- \\
\hline 17 & TX00026 & Red & Tierra Blanca Creek & Randall & Buffalo Lake & $34^{\circ} 55^{\prime} 18^{\prime \prime}$ & $102^{\circ} 06^{\prime} 00^{\prime \prime}$ & 1938 & 575 \\
\hline 18 & TX02317 & Nueces & Trib._Los Tablas Creek & Dimmit & Burro Lake & $28^{\circ} 17^{\prime} 36^{\prime \prime}$ & $99^{\circ} 34^{\prime} 30^{\prime \prime}$ & 1947 & 10 \\
\hline 19 & TX01448 & San Antonio & Calaveras Creek & Bexar & Calaveras Lake & $29^{\circ} 16^{\prime} 42^{\prime \prime}$ & $98^{\circ} 18^{\prime} 18^{\prime \prime}$ & 1969 & 65 \\
\hline 20 & TX02137 & Brazos & Camp Creek & Robertson & Camp Creek Lake & $31^{\circ} 03^{\prime} 42^{\prime \prime}$ & $96^{\circ} 17^{\prime} 12^{\prime \prime}$ & 1949 & 40 \\
\hline 21 & TX00004 & Guadalupe & Guadalupe River & Comal & Canyon Lake & $29^{\circ} 52^{\prime} 00^{\prime \prime}$ & $98^{\circ} 12^{\prime} 00^{\prime \prime}$ & 1964 & 1,425 \\
\hline 22 & TX02267 & Rio Grande & Chacon Creek & Webb & Casa Blanca Lake & $27^{\circ} 32^{\prime} 00^{\prime \prime}$ & $99^{\circ} 26^{\prime} 54^{\prime \prime}$ & 1946 & -- \\
\hline 23 & TX04847 & Trinity-San Jacinto & Cedar Bayou & Chambers & Cedar Bayou Cooling Pond & $29^{\circ} 45^{\prime} 18^{\prime \prime}$ & $94^{\circ} 49^{\prime} 06^{\prime \prime}$ & 1972 & -- \\
\hline
\end{tabular}


Municipal Utility District; MIWA, Municipal and Industrial Water Authority; MWA, Municipal Water Authority; District; MWD, Municipal Water District; SWCD, Soil and Water Conservation District frequency; norm. cap., normal capacity; max. cap., maximum capacity; max. outflow, maximum outflow Commission; $\mathrm{mi}^{2}$, square miles; acre-ft, acre-feet; $\mathrm{ft}^{3} / \mathrm{s}$, cubic feet per second; --, not applicable; $\mathrm{T}$, peak discharge for T-year

\begin{tabular}{|c|c|c|c|c|c|c|c|c|c|c|c|}
\hline $\begin{array}{l}\text { Seq. } \\
\text { no. }\end{array}$ & $\begin{array}{c}\text { Maximum } \\
\text { capacity } \\
\text { (acre-ft) }\end{array}$ & $\begin{array}{l}\text { Normal } \\
\text { capacity } \\
\text { (acre-ft) }\end{array}$ & $\begin{array}{l}\text { Surface } \\
\text { area } \\
\text { (acres) }\end{array}$ & $\begin{array}{c}\text { Maximum } \\
\text { discharge } \\
\left(\mathrm{ft}^{3} / \mathrm{s}\right)\end{array}$ & $\begin{array}{l}\text { Type } \\
\text { of } \\
\text { spill- } \\
\text { way }\end{array}$ & $\begin{array}{l}\text { Pur- } \\
\text { pose } \\
\text { of } \\
\text { dam }\end{array}$ & Owner name & Owner address & $\begin{array}{l}\text { Ques- } \\
\text { tion- } \\
\text { aire } \\
\text { returned }\end{array}$ & $\begin{array}{l}\text { Updated } \\
\text { infor- } \\
\text { mation }\end{array}$ & $\begin{array}{l}\text { Outflow peak- } \\
\text { streamflow } \\
\text { frequency } \\
\left(\mathrm{ft}^{3} / \mathrm{s}\right)\end{array}$ \\
\hline 1 & 204,500 & -- & -- & 9,150 & $\mathrm{C}$ & $\mathrm{FC}$ & $\begin{array}{l}\text { U.S. Army Corps of } \\
\text { Engineers }\end{array}$ & $\begin{array}{l}\text { P.O. Box 1229, Galveston, TX } \\
\quad 77553\end{array}$ & -- & -- & -- \\
\hline 2 & 18,968 & 15,650 & -- & 28,706 & $\mathrm{C}$ & $\mathrm{O}$ & Aluminum Co. of America & $\begin{array}{l}\text { Environmental Superintendent, } \\
\text { Point Comfort, TX } 77978\end{array}$ & -- & -- & -- \\
\hline 3 & $5,289,639$ & $3,151,306$ & 64,438 & $1,507,000$ & $\mathrm{C}$ & $\mathrm{FC}, \mathrm{O}$ & $\begin{array}{l}\text { International Boundary and } \\
\text { Water Commission }\end{array}$ & $\begin{array}{l}\text { U.S. Section, } 4110 \text { Rio Bravo, } \\
\text { El Paso, TX } 79902\end{array}$ & $\mathrm{X}$ & $\begin{array}{l}\text { All but max. } \\
\text { dis. }\end{array}$ & $\begin{array}{l}\mathrm{Q}_{2}=9,700 \\
\mathrm{Q}_{5}=13,000 \\
\mathrm{Q}_{10}=21,000 \\
\mathrm{Q}_{25}=52,000 \\
\mathrm{Q}_{50}=73,000 \\
\mathrm{Q}_{100}=99,000 \\
\mathrm{Q}_{250}=120,000 \\
\mathrm{Q}_{500}=144,000\end{array}$ \\
\hline 4 & 359,900 & 524,00 & -- & 126,800 & $\mathrm{U}$ & $\mathrm{FC}, \mathrm{O}$ & $\begin{array}{l}\text { U.S. Army Corps of } \\
\text { Engineers }\end{array}$ & $\begin{array}{l}\text { P.O. Box 17300, Fort Worth, TX } \\
\quad 76102\end{array}$ & -- & -- & -- \\
\hline 5 & 306,400 & 124,700 & -- & 218,300 & $\mathrm{C}$ & $\mathrm{O}$ & $\begin{array}{l}\text { U.S. Army Corps of } \\
\text { Engineers }\end{array}$ & $\begin{array}{l}\text { P.O. Box 17300, Fort Worth, TX } \\
\quad 76102\end{array}$ & -- & -- & -- \\
\hline 6 & 34,353 & 6,050 & -- & 234,034 & $\mathrm{U}$ & $\mathrm{O}$ & City of Ballinger & P.O. Box 497, Ballinger, TX 76821 & -- & -- & -- \\
\hline 7 & 268,400 & 54,900 & -- & 74,300 & $\mathrm{U}$ & $\mathrm{FC}, \mathrm{O}$ & $\begin{array}{l}\text { U.S. Army Corps of } \\
\text { Engineers }\end{array}$ & $\begin{array}{l}\text { P.O. Box 17300, Fort Worth, TX } \\
\quad 76102\end{array}$ & -- & -- & -- \\
\hline 8 & 207,000 & -- & -- & 10,980 & $\mathrm{C}$ & $\mathrm{FC}$ & $\begin{array}{l}\text { U.S. Army Corps of } \\
\text { Engineers }\end{array}$ & $\begin{array}{l}\text { P.O. Box 1229, Galveston, TX } \\
77553\end{array}$ & -- & -- & -- \\
\hline 9 & 15,950 & 10,400 & 699 & 19,978 & $\mathrm{U}$ & $\mathrm{O}$ & City of Childress & P.O. Box 1087, Childress, TX 79201 & -- & -- & -- \\
\hline 10 & $1,876,700$ & 457,600 & -- & 472,500 & $\mathrm{U}$ & $\mathrm{FC}, \mathrm{O}$ & $\begin{array}{l}\text { U.S. Army Corps of } \\
\text { Engineers }\end{array}$ & $\begin{array}{l}\text { P.O. Box 17300, Fort Worth, TX } \\
\quad 76102\end{array}$ & -- & -- & -- \\
\hline 11 & 410,000 & 88,250 & -- & 172,000 & $\mathrm{U}$ & $\mathrm{FC}, \mathrm{O}$ & $\begin{array}{l}\text { U.S. Army Corps of } \\
\text { Engineers }\end{array}$ & $\begin{array}{l}\text { P.O. Box 17300, Fort Worth, TX } \\
\quad 76102\end{array}$ & -- & -- & -- \\
\hline 12 & 212,400 & 30,000 & -- & 368,990 & $\mathrm{U}$ & $\mathrm{O}$ & City of Brady & P.O. Box 351, Brady, TX 76825 & -- & -- & -- \\
\hline 13 & 38,672 & 29,513 & 1,242 & 1,550 & $\mathrm{U}$ & $\mathrm{O}$ & $\begin{array}{l}\text { Southwestern Electric } \\
\text { Power Co. }\end{array}$ & $\begin{array}{l}\text { P.O. Box 21106, Shreveport, LA } \\
\quad 71156\end{array}$ & -- & -- & -- \\
\hline 14 & 32,324 & 26,500 & 1,350 & 16,944 & $\mathrm{C}$ & $\mathrm{O}$ & City of San Antonio & $\begin{array}{l}512 \text { Mission Rd., San Antonio, TX } \\
78210\end{array}$ & -- & -- & -- \\
\hline 15 & 448,200 & 118,900 & 7,300 & 40,000 & $\mathrm{U}$ & $\mathrm{O}$ & Brown County WID No. 1 & $\begin{array}{l}\text { P.O. Box 118, Brownwood, TX } \\
\quad 76801\end{array}$ & -- & -- & -- \\
\hline 16 & 22,000 & 15,227 & 829 & -- & $\mathrm{C}$ & $\mathrm{O}$ & City of Bryan & P.O. Box 1000, Bryan, TX 77805 & -- & -- & -- \\
\hline 17 & 65,000 & -- & 0 & 209,000 & $\mathrm{U}$ & $\mathrm{O}$ & $\begin{array}{l}\text { U.S. Fish and Wildlife } \\
\text { Service }\end{array}$ & $\begin{array}{l}\text { P.O. Box 1306, Albuquerque, NM } \\
\quad 87103\end{array}$ & -- & -- & -- \\
\hline 18 & 10,000 & 3,500 & $25-50$ & 4,000 & $\mathrm{U}$ & $\mathrm{O}$ & R.W. Briggs, Jr. & P.O. Box 1417, Victoria, TX 77902 & -- & -- & -- \\
\hline 19 & 97,441 & 60,484 & 3,391 & 129,914 & $\mathrm{C}$ & $\mathrm{O}$ & City of San Antonio & $\begin{array}{l}512 \text { Mission Rd., San Antonio, TX } \\
78210\end{array}$ & -- & -- & -- \\
\hline 20 & 25,000 & 8,400 & 750 & 14,300 & $\mathrm{U}$ & $\mathrm{O}$ & Camp Creek Water Co. & -- & -- & -- & -- \\
\hline 21 & $1,129,300$ & 386,200 & -- & 502,800 & $\mathrm{U}$ & $\mathrm{FC}, \mathrm{O}$ & $\begin{array}{l}\text { U.S. Army Corps of } \\
\text { Engineers }\end{array}$ & $\begin{array}{l}\text { P.O. Box 17300, Fort Worth, TX } \\
\quad 76102\end{array}$ & -- & -- & -- \\
\hline 22 & 58,600 & 20,000 & -- & 88,373 & $\mathrm{U}$ & $\mathrm{O}$ & Webb County & P.O. Box 29, Laredo, TX 78040 & -- & -- & -- \\
\hline 23 & 30,000 & 19,250 & -- & -- & $\mathrm{C}$ & $\mathrm{O}$ & $\begin{array}{l}\text { Houston Lighting and } \\
\text { Power Co. }\end{array}$ & P.O. Box 1700, Houston, TX 77001 & -- & -- & -- \\
\hline
\end{tabular}


Table 3. Texas Natural Resource Conservation Commission permitted dams with maximum capacities in excess

\begin{tabular}{|c|c|c|c|c|c|c|c|c|c|}
\hline $\begin{array}{c}\text { Seq. } \\
\text { no. }\end{array}$ & $\begin{array}{c}\text { TNRCC } \\
\text { no. } \\
\text { (pl. 2) }\end{array}$ & $\begin{array}{l}\text { River } \\
\text { basin }\end{array}$ & $\begin{array}{c}\text { Stream } \\
\text { name }\end{array}$ & County & $\begin{array}{c}\text { Reservoir } \\
\text { name }\end{array}$ & Latitude & Longitude & $\begin{array}{c}\text { Year } \\
\text { com- } \\
\text { pleted }\end{array}$ & $\begin{array}{l}\text { Drain- } \\
\text { age } \\
\text { area } \\
\left(\mathrm{mi}^{2}\right)\end{array}$ \\
\hline 24 & TX04380 & Colorado & Cedar Creek & Fayette & Cedar Creek Reservoir & $29^{\circ} 54^{\prime} 54^{\prime \prime}$ & $96^{\circ} 44^{\prime} 12^{\prime \prime}$ & 1977 & 6.3 \\
\hline 25 & TX00237 & Trinity & Cedar Creek & Henderson & Cedar Creek Reservoir & $32^{\circ} 10^{\prime} 48^{\prime \prime}$ & $96^{\circ} 04^{\prime} 06^{\prime \prime}$ & 1966 & 1,007 \\
\hline 26 & TX01691 & Colorado & Champion Creek & Mitchell & Champion Creek Reservoir & $32^{\circ} 16 ' 54^{\prime \prime}$ & $100^{\circ} 51^{\prime} 36^{\prime \prime}$ & 1959 & 164 \\
\hline 27 & TX04425 & Nueces & Frio River & Live Oak & Choke Canyon Reservoir & $28^{\circ} 29^{\prime} 06^{\prime \prime}$ & $98^{\circ} 14^{\prime} 36^{\prime \prime}$ & 1982 & -- \\
\hline 28 & TX00419 & Red & Coffee Mill Creek & Fannin & Coffee Mill Lake & $33^{\circ} 44^{\prime} 06^{\prime \prime}$ & $95^{\circ} 58^{\prime} 00^{\prime \prime}$ & 1938 & -- \\
\hline 29 & TX04744 & Guadalupe & Coleto Creek & Victoria & Coleto Creek Cooling Pond & $28^{\circ} 43^{\prime} 24^{\prime \prime}$ & $97^{\circ} 10^{\prime} 00^{\prime \prime}$ & 1980 & 494 \\
\hline 30 & TX08012 & Red & South Sulphur River & Hopkins & Cooper Lake & $33^{\circ} 19^{\prime} 48^{\prime \prime}$ & $95^{\circ} 37^{\prime} 30^{\prime \prime}$ & 1991 & 476 \\
\hline 31 & TX03790 & Nueces-Rio Grande & Off-channel & Hidalgo & Delta Lake 2 & $26^{\circ} 25^{\prime} 48^{\prime \prime}$ & $97^{\circ} 56^{\prime} 12^{\prime \prime}$ & 1939 & 3.01 \\
\hline 32 & TX00779 & Trinity & West Fork Trinity River & Tarrant & Eagle Mountain Reservoir & $32^{\circ} 52^{\prime} 12^{\prime \prime}$ & $97^{\circ} 29^{\prime} 48^{\prime \prime}$ & 1932 & 1,970 \\
\hline 33 & TX04010 & Cypress & Ellison Creek & Morris & Ellison Creek Reservoir & $32^{\circ} 55^{\prime} 06^{\prime \prime}$ & $94^{\circ} 43^{\prime} 30^{\prime \prime}$ & 1943 & 37 \\
\hline 34 & TX03517 & Colorado & Colorado River & Coke & E.V. Spence Reservoir & $31^{\circ} 53^{\prime} 42^{\prime \prime}$ & $100^{\circ} 30^{\prime} 54^{\prime \prime}$ & 1969 & 4,140 \\
\hline 35 & TX00692 & Trinity & Big Brown Creek & Freestone & Fairfield Lake & $31^{\circ} 49^{\prime} 06^{\prime \prime}$ & $96^{\circ} 02^{\prime} 30^{\prime \prime}$ & 1969 & -- \\
\hline 36 & TX04395 & Trinity & Caney Creek & Henderson & Forest Grove Reservoir & $32^{\circ} 13^{\prime} 42^{\prime \prime}$ & $95^{\circ} 57^{\prime} 54^{\prime \prime}$ & 1980 & -- \\
\hline 37 & TX01054 & Brazos & Navasota River & Limestone & Fort Parker State Park Lake & $31^{\circ} 35^{\prime} 18^{\prime \prime}$ & $96^{\circ} 31^{\prime} 36^{\prime \prime}$ & 1939 & -- \\
\hline 38 & TX02483 & Brazos & Big Elm Creek & Jones & Fort Phantom Hill Lake & $32^{\circ} 37^{\prime} 00^{\prime \prime}$ & $99^{\circ} 40^{\prime} 06^{\prime \prime}$ & 1938 & 463 \\
\hline 39 & TX04634 & Brazos & Gibbons Creek & Grimes & Gibbons Creek Reservoir & $30^{\circ} 36^{\prime} 36^{\prime \prime}$ & $96^{\circ} 03^{\prime} 42^{\prime \prime}$ & 1981 & 85 \\
\hline 40 & TX08005 & Brazos & San Gabriel River & Williamson & Granger Lake & $30^{\circ} 42^{\prime} 12^{\prime \prime}$ & $97^{\circ} 19^{\prime} 00^{\prime \prime}$ & 1979 & -- \\
\hline 41 & TX03698 & Red & Salt Fork Red River & Donley & Greenbelt Reservoir & $35^{\circ} 00^{\prime} 06^{\prime \prime}$ & $100^{\circ} 53^{\prime} 36^{\prime \prime}$ & 1968 & 266 \\
\hline 42 & TX01912 & Guadalupe & Guadalupe River & Gonzales & H-4 Lake & $29^{\circ} 29^{\prime} 42^{\prime \prime}$ & $97^{\circ} 37^{\prime} 30^{\prime \prime}$ & 1931 & 1,917 \\
\hline 43 & TX00006 & Colorado & Hords Creek & Coleman & Hords Creek Reservoir & $31^{\circ} 51^{\prime} 00^{\prime \prime}$ & $99^{\circ} 34^{\prime} 00^{\prime \prime}$ & 1948 & -- \\
\hline 44 & TX03268 & Trinity & Little Elkhart Creek & Houston & Houston County Lake & $31^{\circ} 24^{\prime} 24^{\prime \prime}$ & $95^{\circ} 36^{\prime} 18^{\prime \prime}$ & 1966 & 44 \\
\hline 45 & TX03639 & Brazos & Hubbard Creek & Stephens & Hubbard Creek Reservoir & $32^{\circ} 49^{\prime} 42^{\prime \prime}$ & $98^{\circ} 57^{\prime} 48^{\prime \prime}$ & 1962 & 1,107 \\
\hline 46 & TX00579 & Red & Fish Creek & Cooke & Hubert M. Moss Lake & $33^{\circ} 46^{\prime} 24^{\prime \prime}$ & $97^{\circ} 12^{\prime} 48^{\prime \prime}$ & 1966 & -- \\
\hline 47 & TX00988 & Colorado & Colorado River & Burnet & Inks Lake & $30^{\circ} 43^{\prime} 48^{\prime \prime}$ & $98^{\circ} 23^{\prime} 06^{\prime \prime}$ & 1938 & -- \\
\hline 48 & TX00024 & Rio Grande & Rio Grande & Starr & International Falcon Reservoir & $26^{\circ} 33^{\prime} 30^{\prime \prime}$ & $99^{\circ} 09^{\prime} 42^{\prime \prime}$ & 1954 & 159,269 \\
\hline 49 & TX08007 & Trinity & Mountain Creek & Dallas & Joe Pool Lake & $32^{\circ} 38^{\prime} 42^{\prime \prime}$ & $96^{\circ} 59^{\prime} 36^{\prime \prime}$ & 1986 & -- \\
\hline 50 & TX03887 & Cypress & Johnson Creek & Marion & Johnson Creek Reservoir & $32^{\circ} 50^{\prime} 24^{\prime \prime}$ & $94^{\circ} 32^{\prime} 54^{\prime \prime}$ & 1961 & -- \\
\hline 51 & TX00204 & Trinity & Coon Creek & Henderson & Coon Creek Lake & $32^{\circ} 02^{\prime} 18^{\prime \prime}$ & $95^{\circ} 51^{\prime} 30^{\prime \prime}$ & 1934 & -- \\
\hline
\end{tabular}




\begin{tabular}{|c|c|c|c|c|c|c|c|c|c|c|c|}
\hline $\begin{array}{l}\text { Seq. } \\
\text { no. }\end{array}$ & $\begin{array}{l}\text { Maximum } \\
\text { capacity } \\
\text { (acre-ft) }\end{array}$ & $\begin{array}{l}\text { Normal } \\
\text { capacity } \\
\text { (acre-ft) }\end{array}$ & $\begin{array}{l}\text { Surface } \\
\text { area } \\
\text { (acres) }\end{array}$ & $\begin{array}{l}\text { Maximum } \\
\text { discharge } \\
\left(\mathrm{ft}^{3} / \mathrm{s}\right)\end{array}$ & $\begin{array}{l}\text { Type } \\
\text { of } \\
\text { spill- } \\
\text { way }\end{array}$ & $\begin{array}{l}\text { Pur- } \\
\text { pose } \\
\text { of } \\
\text { dam }\end{array}$ & Owner name & Owner address & $\begin{array}{l}\text { Ques- } \\
\text { tion- } \\
\text { aire } \\
\text { returned }\end{array}$ & $\begin{array}{l}\text { Updated } \\
\text { infor- } \\
\text { mation }\end{array}$ & $\begin{array}{l}\text { Outflow peak- } \\
\text { streamflow } \\
\text { frequency } \\
\left(\mathrm{ft}^{3} / \mathrm{s}\right)\end{array}$ \\
\hline 24 & 88,628 & 71,400 & 2,400 & 1,152 & $\mathrm{U}$ & $\mathrm{O}$ & $\begin{array}{l}\text { Lower Colorado River } \\
\text { Authority }\end{array}$ & P.O. Box 220, Austin, TX 78767 & $\mathrm{X}$ & -- & -- \\
\hline 25 & $1,085,000$ & 679,200 & 25,850 & 316,821 & $\mathrm{C}$ & $\mathrm{O}$ & Tarrant County WCID No. 1 & $\begin{array}{l}\text { P.O. Box 4508, Fort Worth, TX } \\
76102\end{array}$ & -- & -- & -- \\
\hline 26 & 90,200 & 42,500 & -- & 85,290 & $\mathrm{U}$ & $\mathrm{O}$ & TU Electric & $\begin{array}{l}\text { Skyway Tower, } 400 \text { N. Olive St., } \\
\text { Dallas, TX } 75201\end{array}$ & -- & -- & -- \\
\hline 27 & $1,083,000$ & 714,000 & -- & 250,584 & $\mathrm{C}$ & $\mathrm{O}$ & Bureau of Reclamation & P.O. Box 1946, Austin, TX 78767 & -- & -- & -- \\
\hline 28 & 24,070 & 8,000 & -- & 3,400 & $\mathrm{U}$ & $\mathrm{O}$ & U.S. Forest Service & P.O. Box 969, Lufkin, TX 75901 & -- & -- & -- \\
\hline 29 & 169,000 & 35,084 & 3,100 & 415,300 & $\mathrm{U}$ & $\mathrm{O}$ & $\begin{array}{l}\text { Guadalupe-Blanco River } \\
\text { Authority }\end{array}$ & P.O. Box 271, Seguin, TX 78155 & $\mathrm{X}$ & DA; SA & -- \\
\hline 30 & 797,300 & 310,000 & 19,280 & 135,600 & $\mathrm{U}$ & $\mathrm{O}$ & $\begin{array}{l}\text { U.S. Army Corps of } \\
\text { Engineers }\end{array}$ & $\begin{array}{l}\text { P.O. Box 17300, Fort Worth, TX } \\
\quad 76102\end{array}$ & -- & -- & -- \\
\hline 31 & 22,545 & 17,788 & 1,227 & -- & -- & $\mathrm{O}$ & $\begin{array}{l}\text { Delta Lake Irrigation } \\
\text { District }\end{array}$ & Rt. 1, Box 225, Edcouch, TX 78538 & -- & -- & -- \\
\hline 32 & 680,335 & 190,460 & 6,160 & 125,100 & $\mathrm{C}$ & $\mathrm{O}$ & Tarrant County WCID No. 1 & $\begin{array}{l}\text { P.O. Box 4508, Fort Worth, TX } \\
\quad 76102\end{array}$ & -- & -- & -- \\
\hline 33 & 36,000 & 24,700 & 1,500 & 39,149 & $\mathrm{U}$ & $\mathrm{O}$ & Lone Star Steel Co. & P.O. Box 1000, Lone Star, TX 75668 & $\mathrm{X}$ & $\begin{array}{l}\text { Address; ph.; } \\
\text { DA; SA }\end{array}$ & -- \\
\hline 34 & 810,000 & 488,760 & 18,000 & 796,007 & $\mathrm{U}$ & $\mathrm{O}$ & Colorado River MUD & P.O. Box 869, Big Spring, TX 79721 & -- & -- & -- \\
\hline 35 & 70,840 & 50,600 & -- & 32,260 & $\mathrm{U}$ & $\mathrm{O}$ & TU Electric & $\begin{array}{l}\text { Skyway Tower, } 400 \text { N. Olive St., } \\
\text { Dallas, TX } 75201\end{array}$ & -- & -- & -- \\
\hline 36 & 54,300 & 20,038 & -- & 104,900 & $\mathrm{U}$ & $\mathrm{O}$ & TU Electric & $\begin{array}{l}\text { Skyway Tower, } 400 \text { N. Olive St., } \\
\text { Dallas, TX } 75201\end{array}$ & -- & -- & -- \\
\hline 37 & 21,274 & 3,100 & -- & 59,929 & $\mathrm{U}$ & $\mathrm{O}$ & $\begin{array}{l}\text { Fort Parker State Recreation } \\
\quad \text { Area }\end{array}$ & Rt. 3, Box 95, Mexia, TX 76667 & -- & -- & -- \\
\hline 38 & 127,000 & 73,960 & 4,246 & 578,139 & $\mathrm{U}$ & $\mathrm{O}$ & City of Abilene & P.O. Box 60, Abilene, TX 79604 & $\mathrm{X}$ & Dis. freq. & $\begin{array}{l}\mathrm{Q}_{2}=3,400 \\
\mathrm{Q}_{5}=7,500 \\
\mathrm{Q}_{10}=11,800 \\
\mathrm{Q}_{25}=10,000 \\
\mathrm{Q}_{50}=21,600 \\
\mathrm{Q}_{500}=578,139\end{array}$ \\
\hline 39 & 81,874 & 32,084 & 2,770 & 26,000 & $\mathrm{U}$ & $\mathrm{O}$ & $\begin{array}{l}\text { Texas Municipal Power } \\
\text { Agency }\end{array}$ & P.O. Box 7000 Bryan, TX 77805 & -- & -- & -- \\
\hline 40 & 561,100 & 65,500 & -- & 342,330 & $\mathrm{U}$ & $\mathrm{FC}, \mathrm{O}$ & $\begin{array}{l}\text { U.S. Army Corps of } \\
\text { Engineers }\end{array}$ & $\begin{array}{l}\text { P.O. Box 17300, Fort Worth, TX } \\
\quad 76102\end{array}$ & -- & -- & -- \\
\hline 41 & 100,500 & 59,800 & 2,020 & 313,545 & $\mathrm{U}$ & $\mathrm{O}$ & Greenbelt MIWA & P.O. Box 665, Clarendon, TX 79226 & -- & -- & -- \\
\hline 42 & 23,520 & 4,620 & 495 & -- & $\mathrm{C}$ & $\mathrm{O}$ & $\begin{array}{l}\text { Guadalupe-Blanco River } \\
\text { Authority }\end{array}$ & P.O. Box 271, Seguin, TX 78155 & $\mathrm{x}$ & $\begin{array}{l}\text { DA; norm. } \\
\text { cap.; SA }\end{array}$ & -- \\
\hline 43 & 49,290 & 8,640 & -- & 61,700 & $\mathrm{U}$ & $\mathrm{FC}, \mathrm{O}$ & $\begin{array}{l}\text { U.S. Army Corps of } \\
\text { Engineers }\end{array}$ & $\begin{array}{l}\text { P.O. Box 17300, Fort Worth, TX } \\
\quad 76102\end{array}$ & -- & -- & -- \\
\hline 44 & 27,000 & 19,500 & 1,498 & 38,150 & $\mathrm{U}$ & $\mathrm{O}$ & $\begin{array}{l}\text { Houston County WCID } \\
\text { No. } 1\end{array}$ & P.O. Box 1246, Crockett, TX 75835 & $\mathrm{X}$ & $\mathrm{DA} ; \mathrm{SA}$ & -- \\
\hline 45 & 720,000 & 317,750 & 15,250 & 480,387 & $\mathrm{U}$ & $\mathrm{O}$ & West Central Texas MUD & P.O. Box 2362, Abilene, TX 79604 & -- & -- & -- \\
\hline 46 & 55,000 & 23,210 & -- & 114858 & $\mathrm{U}$ & $\mathrm{O}$ & City of Gainesville & 200 S. Rusk, Gainesville, TX 76240 & -- & -- & -- \\
\hline 47 & 63,500 & 17,545 & 803 & $1,207,697$ & $\mathrm{U}$ & $\mathrm{O}$ & $\begin{array}{l}\text { Lower Colorado River } \\
\text { Authority }\end{array}$ & P.O. Box 220, Austin, TX 78767 & $\mathrm{x}$ & -- & -- \\
\hline 48 & $3,964,541$ & $2,653,793$ & 87,181 & 490,000 & $\mathrm{C}$ & $\mathrm{FC}, \mathrm{O}$ & $\begin{array}{l}\text { International Boundary and } \\
\text { Water Commission }\end{array}$ & $\begin{array}{l}\text { U.S. Section, } 4110 \text { Rio Bravo, } \\
\text { El Paso, TX } 79902\end{array}$ & $\mathrm{X}$ & $\begin{array}{l}\text { All but max. } \\
\text { dis. }\end{array}$ & $\begin{array}{l}\mathrm{Q}_{2}=13,700 \\
\mathrm{Q}_{5}=16,300 \\
\mathrm{Q}_{10}=22,500 \\
\mathrm{Q}_{25}=55,000 \\
\mathrm{Q}_{50}=80,000 \\
\mathrm{Q}_{100}=110,000 \\
\mathrm{Q}_{250}=165,000 \\
\mathrm{Q}_{500}=200,000\end{array}$ \\
\hline 49 & 642,400 & 176,900 & -- & -- & $\mathrm{U}$ & $\mathrm{FC}, \mathrm{O}$ & $\begin{array}{l}\text { U.S. Army Corps of } \\
\text { Engineers }\end{array}$ & $\begin{array}{l}\text { P.O. Box 17300, Fort Worth, TX } \\
\quad 76102\end{array}$ & -- & -- & -- \\
\hline 50 & 21,716 & 10,038 & -- & 14,639 & $\mathrm{U}$ & $\mathrm{O}$ & $\begin{array}{l}\text { Southwestern Electric } \\
\text { Power Co. }\end{array}$ & $\begin{array}{l}\text { P.O. Box 21106, Shreveport, LA } \\
\quad 71156\end{array}$ & -- & -- & -- \\
\hline 51 & 21,366 & 3,631 & -- & -- & $\mathrm{U}$ & $\mathrm{O}$ & Coon Creek Club & P.O. Box 20297, Dallas, TX 75220 & -- & -- & -- \\
\hline
\end{tabular}


Table 3. Texas Natural Resource Conservation Commission permitted dams with maximum capacities in excess

\begin{tabular}{|c|c|c|c|c|c|c|c|c|c|}
\hline $\begin{array}{l}\text { Seq. } \\
\text { no. }\end{array}$ & $\begin{array}{c}\text { TNRCC } \\
\text { no. } \\
\text { (pl. 2) }\end{array}$ & $\begin{array}{l}\text { River } \\
\text { basin }\end{array}$ & $\begin{array}{l}\text { Stream } \\
\text { name }\end{array}$ & County & $\begin{array}{l}\text { Reservoir } \\
\text { name }\end{array}$ & Latitude & Longitude & $\begin{array}{l}\text { Year } \\
\text { com- } \\
\text { pleted }\end{array}$ & $\begin{array}{l}\text { Drain- } \\
\text { age } \\
\text { area } \\
\left(\mathrm{mi}^{2}\right)\end{array}$ \\
\hline 52 & TX06464 & Brazos & $\begin{array}{l}\text { Double Mountain Fork Brazos } \\
\text { River }\end{array}$ & Garza & Lake Alan Henry & $33^{\circ} 03^{\prime} 41^{\prime \prime}$ & $101^{\circ} 02^{\prime} 30^{\prime \prime}$ & 1994 & 394 \\
\hline 53 & TX00699 & Trinity & Big Sandy Creek & Montague & Lake Amon G. Carter & $33^{\circ} 28^{\prime} 06^{\prime \prime}$ & $97^{\circ} 51^{\prime} 54^{\prime \prime}$ & 1956 & -- \\
\hline 54 & TX09088 & Trinity & Turtle Bayou & Chambers & Lake Anahuac & $29^{\circ} 46^{\prime} 48^{\prime \prime}$ & $94^{\circ} 41^{\prime} 38^{\prime \prime}$ & 1954 & 199 \\
\hline
\end{tabular}

\begin{tabular}{|c|c|c|c|c|c|c|c|c|c|}
\hline 55 & TX00776 & Trinity & Village Creek & Tarrant & Lake Arlington & $32^{\circ} 43^{\prime} 18^{\prime \prime}$ & $97^{\circ} 11^{\prime} 54^{\prime \prime}$ & 1955 & 143 \\
\hline 56 & TX02883 & Red & Little Wichita River & Clay & Lake Arrowhead & $33^{\circ} 45^{\prime} 42^{\prime \prime}$ & $98^{\circ} 22^{\prime} 00^{\prime \prime}$ & 1966 & 1.28 \\
\hline 57 & TX00182 & Neches & Flat Creek & Henderson & Lake Athens & $32^{\circ} 12^{\prime} 18^{\prime \prime}$ & $95^{\circ} 43^{\prime} 24^{\prime \prime}$ & 1963 & 21.1 \\
\hline 58 & TX01086 & Colorado & Colorado River & Travis & Lake Austin & $30^{\circ} 17^{\prime} 42^{\prime \prime}$ & $97^{\circ} 47^{\prime} 12^{\prime \prime}$ & 1939 & -- \\
\hline 59 & TX02718 & Colorado & Spicer Creek & Bastrop & Lake Bastrop & $30^{\circ} 09^{\prime} 18^{\prime \prime}$ & $97^{\circ} 17^{\prime} 30^{\prime \prime}$ & 1964 & 8.7 \\
\hline 60 & TX04785 & Cypress & Big Cypress Creek & Titus & Lake Bob Sandlin & $33^{\circ} 04^{\prime} 30^{\prime \prime}$ & $95^{\circ} 00^{\prime} 06^{\prime \prime}$ & 1978 & 239 \\
\hline 61 & TX00402 & Red & Timber Creek & Fannin & Lake Bonham & $33^{\circ} 39^{\prime} 06^{\prime \prime}$ & $96^{\circ} 07^{\prime} 48^{\prime \prime}$ & 1969 & -- \\
\hline 62 & TX04101 & Brazos & Brazos River & McLennan & Lake Brazos & $31^{\circ} 33^{\prime} 06^{\prime \prime}$ & $97^{\circ} 05^{\prime} 42^{\prime \prime}$ & 1970 & -- \\
\hline 63 & TX01496 & Trinity & West Fork Trinity River & Wise & Lake Bridgeport & $33^{\circ} 13^{\prime} 12^{\prime \prime}$ & $97^{\circ} 49^{\prime} 48^{\prime \prime}$ & 1931 & 1,111 \\
\hline 64 & TX00989 & Colorado & Colorado River & Burnet & Lake Buchanan & $30^{\circ} 45^{\prime} 06^{\prime \prime}$ & $98^{\circ} 25^{\prime} 06^{\prime \prime}$ & 1937 & 50.06 \\
\hline 65 & TX03453 & Sabine & Cherokee Bayou & Gregg & Lake Cherokee & $32^{\circ} 21^{\prime} 42^{\prime \prime}$ & $94^{\circ} 36^{\prime} 18^{\prime \prime}$ & 1948 & 167 \\
\hline 66 & TX01409 & Brazos & Sandy Creek & Eastland & Lake Cisco & $32^{\circ} 26^{\prime} 24^{\prime \prime}$ & $98^{\circ} 59^{\prime} 00^{\prime \prime}$ & 1923 & -- \\
\hline 67 & TX02152 & Colorado & Jim Ned Creek & Coleman & Lake Coleman & $32^{\circ} 01^{\prime} 48^{\prime \prime}$ & $99^{\circ} 27^{\prime} 54^{\prime \prime}$ & 1966 & 299 \\
\hline 68 & TX01693 & Colorado & Morgan Creek & Mitchell & Lake Colorado City & $32^{\circ} 19^{\prime} 06^{\prime \prime}$ & $100^{\circ} 55^{\prime} 00^{\prime \prime}$ & 1949 & 322 \\
\hline 69 & TX03491 & Brazos & Mercer Creek & Comanche & Lake Comanche & $31^{\circ} 48^{\prime} 06^{\prime \prime}$ & $98^{\circ} 34^{\prime} 42^{\prime \prime}$ & 1948 & 37.8 \\
\hline 70 & TX00097 & San Jacinto & West Fork San Jacinto River & Montgomery & Lake Conroe & $30^{\circ} 21^{\prime} 24^{\prime \prime}$ & $95^{\circ} 33^{\prime} 36^{\prime \prime}$ & 1973 & 444 \\
\hline 71 & TX03895 & Nueces & Nueces River & Jim Wells & Lake Corpus Christi & $28^{\circ} 02^{\prime} 30^{\prime \prime}$ & $97^{\circ} 51^{\prime} 54 "$ & 1958 & -- \\
\hline 72 & TX04066 & Brazos & Manos Creek & McLennan & Lake Creek Lake & $31^{\circ} 27^{\prime} 24^{\prime \prime}$ & $96^{\circ} 59^{\prime} 12^{\prime \prime}$ & 1953 & -- \\
\hline 73 & TX01841 & Red & Pine Creek & Lamar & Lake Crook & $33^{\circ} 43^{\prime} 42^{\prime \prime}$ & $95^{\circ} 34^{\prime} 00^{\prime \prime}$ & 1923 & 53.06 \\
\hline 74 & TX03288 & Cypress & Big Cypress Creek & Franklin & Lake Cypress Springs & $33^{\circ} 03^{\prime} 24^{\prime \prime}$ & $95^{\circ} 08^{\prime} 24^{\prime \prime}$ & 1971 & 75 \\
\hline 75 & TX03635 & Brazos & Gonzales Creek & Stephens & Lake Daniel & $32^{\circ} 38^{\prime} 54^{\prime \prime}$ & $98^{\circ} 52^{\prime} 06^{\prime \prime}$ & 1948 & 115 \\
\hline 76 & TX01424 & Brazos & Dutchman Creek & Knox & Lake Davis & $33^{\circ} 31^{\prime} 24^{\prime \prime}$ & $99^{\circ} 44^{\prime} 30^{\prime \prime}$ & 1959 & -- \\
\hline 77 & TX01011 & Red & Wichita River & Archer & Lake Diverson & $33^{\circ} 49^{\prime} 12^{\prime \prime}$ & $98^{\circ} 56^{\prime} 12^{\prime \prime}$ & 1924 & 3.47 \\
\hline 78 & TX03946 & Brazos & Flint Creek & Young & Lake Eddleman & $33^{\circ} 07^{\prime} 48^{\prime \prime}$ & $98^{\circ} 36^{\prime} 30^{\prime \prime}$ & 1929 & -- \\
\hline 79 & TX00966 & Red & Camp Creek & Wilbarger & Lake Electra & $33^{\circ} 58^{\prime} 30^{\prime \prime}$ & $99^{\circ} 01 ' 24 "$ & 1950 & -- \\
\hline 80 & TX04388 & Sabine & Lake Fork Creek & Wood & Lake Fork Reservoir & $32^{\circ} 48^{\prime} 24^{\prime \prime}$ & $95^{\circ} 32^{\prime} 24^{\prime \prime}$ & 1980 & 493 \\
\hline 81 & TX08006 & Brazos & North Fork San Gabriel River & Williamson & Lake Georgetown & $30^{\circ} 40^{\prime} 30^{\prime \prime}$ & $97^{\circ} 43^{\prime} 30^{\prime \prime}$ & 1980 & 246 \\
\hline 82 & TX06485 & Cypress & Kelsey Creek & Upshur & Lake Gilmer & $32^{\circ} 45^{\prime} 48^{\prime \prime}$ & $94^{\circ} 58^{\prime} 54^{\prime \prime}$ & 1996 & 35.6 \\
\hline 83 & TX03725 & Sabine & Glade Creek & Upshur & Lake Gladewater & $32^{\circ} 33^{\prime} 18^{\prime \prime}$ & $94^{\circ} 57^{\prime} 30^{\prime \prime}$ & 1952 & -- \\
\hline
\end{tabular}




\begin{tabular}{|c|c|c|c|c|c|c|c|c|c|c|c|}
\hline $\begin{array}{l}\text { Seq. } \\
\text { no. }\end{array}$ & $\begin{array}{l}\text { Maximum } \\
\text { capacity } \\
\text { (acre-ft) }\end{array}$ & $\begin{array}{l}\text { Normal } \\
\text { capacity } \\
\text { (acre-ft) }\end{array}$ & $\begin{array}{l}\text { Surface } \\
\text { area } \\
\text { (acres) }\end{array}$ & $\begin{array}{l}\text { Maximum } \\
\text { discharge } \\
\left(\mathrm{ft}^{3} / \mathrm{s}\right)\end{array}$ & $\begin{array}{l}\text { Type } \\
\text { of } \\
\text { spill- } \\
\text { way }\end{array}$ & $\begin{array}{l}\text { Pur- } \\
\text { pose } \\
\text { of } \\
\text { dam }\end{array}$ & Owner name & Owner address & $\begin{array}{l}\text { Ques- } \\
\text { tion- } \\
\text { aire } \\
\text { returned }\end{array}$ & $\begin{array}{l}\text { Updated } \\
\text { infor- } \\
\text { mation }\end{array}$ & $\begin{array}{l}\text { Outflow peak- } \\
\text { streamflow } \\
\text { frequency } \\
\left(\mathrm{ft}^{3} / \mathrm{s}\right)\end{array}$ \\
\hline 52 & 354,500 & 115,937 & 2,884 & 360,000 & $\mathrm{U}$ & $\mathrm{O}$ & Brazos River Authority & P.O. Box 7555, Waco, TX 76714 & $\mathrm{X}$ & Max. dis. & -- \\
\hline 53 & 58,050 & 20,050 & -- & 149,460 & $\mathrm{U}$ & $\mathrm{O}$ & City of Bowie & 304 Lindsey St., Bowie, TX 76230 & -- & -- & -- \\
\hline 54 & 60,000 & 35,300 & 5,300 & 17,200 & $\mathrm{U}$ & $\mathrm{O}$ & $\begin{array}{l}\text { Chambers-Liberty Counties } \\
\text { Navigation District }\end{array}$ & P.O. Box 518, Anahuac, TX 77514 & $\mathrm{X}$ & $\begin{array}{l}\text { Gen. Man., } \\
\text { Pudge } \\
\text { Willcox; } \\
\text { DA; yr. } \\
\text { completed; } \\
\text { SA; max } \\
\text { dis. }\end{array}$ & -- \\
\hline 55 & 103,500 & 45,710 & 2,275 & 44,120 & $\mathrm{C}$ & $\mathrm{O}$ & City of Arlington & P.O. Box 231, Arlington, TX 76004 & -- & -- & -- \\
\hline 56 & 685,000 & 262,100 & 16,200 & 270,700 & $\mathrm{U}$ & $\mathrm{O}$ & City of Wichita Falls & $\begin{array}{l}\text { P.O. Box 1431, Wichita Falls, TX } \\
\quad 76307\end{array}$ & -- & -- & -- \\
\hline 57 & 47,000 & 32,840 & 2,432 & 14,291 & $\mathrm{U}$ & $\mathrm{O}$ & Athens MWA & 501 N. Pinkerton, Athens, TX 75751 & -- & -- & -- \\
\hline 58 & 73,100 & 21,000 & -- & $1,382,697$ & $\mathrm{U}$ & $\mathrm{O}$ & City of Austin & P.O. Box 1088, Austin, TX 78767 & $\mathrm{X}$ & -- & -- \\
\hline 59 & 16,962 & 16,590 & 244 & 17,612 & $\mathrm{C}$ & $\mathrm{O}$ & $\begin{array}{l}\text { Lower Colorado River } \\
\text { Authority }\end{array}$ & P.O. Box 220, Austin, TX 78767 & $\mathrm{X}$ & -- & -- \\
\hline 60 & 297,490 & 204,678 & 9,004 & 249,300 & $\mathrm{C}$ & $\mathrm{O}$ & Titus County FWSD No. 1 & $\begin{array}{l}\text { P.O. Box 650, Mount Pleasant, TX } \\
75455\end{array}$ & $\mathrm{x}$ & All but DA & -- \\
\hline 61 & 28,000 & 13,000 & -- & 14,600 & $\mathrm{U}$ & $\mathrm{O}$ & Bonham MWA & $\begin{array}{l}2016 \text { Arbor Bend, Bonham, TX } \\
75418\end{array}$ & -- & -- & -- \\
\hline 62 & 10,000 & 3,537 & -- & 100,000 & $\mathrm{C}$ & $\mathrm{O}$ & City of Waco & P.O. Box 2570, Waco, TX 76702 & -- & -- & -- \\
\hline 63 & 923,814 & 386,539 & 12,940 & 99,600 & $\mathrm{C}$ & $\mathrm{FC}, \mathrm{O}$ & Tarrant County WCID No. 1 & $\begin{array}{l}\text { P.O. Box 4508, Fort Worth, TX } \\
\quad 76102\end{array}$ & -- & -- & -- \\
\hline 64 & 982,000 & 846,303 & 23,060 & $1,339,388$ & $\mathrm{U}$ & $\mathrm{O}$ & $\begin{array}{l}\text { Lower Colorado River } \\
\text { Authority }\end{array}$ & P.O. Box 220, Austin, TX 78767 & $\mathrm{X}$ & -- & -- \\
\hline 65 & 101,041 & 62,400 & 3,083 & 120,000 & $\mathrm{U}$ & $\mathrm{O}$ & Cherokee Water Co. & $\begin{array}{l}\text { NK-21 Lake Cherokee, Longview, } \\
\text { TX } 75603\end{array}$ & $\mathrm{x}$ & DA; SA & -- \\
\hline 66 & 45,000 & 45,000 & -- & 25,600 & $\mathrm{U}$ & $\mathrm{O}$ & City of Cisco & P.O. Box 110, Cisco, TX 76437 & -- & -- & -- \\
\hline 67 & 91,680 & 38,846 & 1,886 & 265,923 & $\mathrm{U}$ & $\mathrm{O}$ & City of Coleman & P.O. Box 592, Coleman, TX 76834 & -- & -- & -- \\
\hline 68 & 70,700 & 31,800 & 1,610 & 150,000 & $\mathrm{U}$ & $\mathrm{O}$ & TU Electric & $\begin{array}{l}\text { Skyway Tower, } 400 \text { N. Olive St., } \\
\text { Dallas, TX } 75201\end{array}$ & -- & -- & -- \\
\hline 69 & 10,634 & 4,800 & 382 & 100,019 & $\mathrm{U}$ & $\mathrm{O}$ & Jimmy E. and Walter J. Gore & P.O. Box 274, Comanche, TX 76442 & -- & -- & -- \\
\hline 70 & 706,970 & 430,260 & 20,985 & 247,247 & $\mathrm{C}$ & $\mathrm{O}$ & San Jacinto River Authority & P.O. Box 329, Conroe, TX 77305 & -- & -- & -- \\
\hline 71 & 531,000 & 300,000 & -- & $1,853,758$ & $\mathrm{C}$ & $\mathrm{O}$ & City of Corpus Christi & P.O. Box 98, Sandia, TX 78383 & -- & -- & -- \\
\hline 72 & 10,580 & 8,500 & -- & 42,000 & $\mathrm{U}$ & $\mathrm{O}$ & TU Electric & $\begin{array}{l}\text { Skyway Tower, } 400 \text { N. Olive St., } \\
\text { Dallas, TX } 75201\end{array}$ & -- & -- & -- \\
\hline 73 & 27,600 & 11,011 & 890 & 30,794 & $\mathrm{U}$ & $\mathrm{O}$ & City of Paris & P.O. Box 9037, Paris, TX 75461 & $\mathrm{x}$ & DA; SA & -- \\
\hline 74 & 164,000 & 72,800 & 4,750 & 69,257 & -- & $\mathrm{O}$ & $\begin{array}{l}\text { Franklin County Water } \\
\text { District }\end{array}$ & $\begin{array}{l}\text { P.O. Box 559, Mount Vernon, TX } \\
75457\end{array}$ & -- & -- & -- \\
\hline 75 & 38,242 & 11,400 & 954 & 222,020 & $\mathrm{U}$ & $\mathrm{O}$ & City of Breckenridge & $\begin{array}{l}209 \text { N. Breckenridge Ave., } \\
\text { Breckenridge, TX } 76424\end{array}$ & -- & -- & -- \\
\hline 76 & 19,000 & 5,395 & -- & -- & $\mathrm{U}$ & $\mathrm{O}$ & Eagle Ranch Inc. & -- & -- & -- & -- \\
\hline 77 & 144,162 & 33,420 & 3,133 & 377,626 & $\mathrm{U}$ & $\mathrm{FC}, \mathrm{O}$ & $\begin{array}{l}\text { Wichita County WCID } \\
\text { No. } 2 \text { and City of Wichita } \\
\text { Falls }\end{array}$ & $\begin{array}{l}402 \text { E. Scott St., Wichita Falls, TX } \\
76301\end{array}$ & -- & -- & -- \\
\hline 78 & 35,000 & 13,386 & -- & -- & $\mathrm{U}$ & $\mathrm{O}$ & City of Graham & P.O. Box 1449, Graham, TX 76450 & -- & -- & -- \\
\hline 79 & 21,370 & 8,730 & -- & 20,687 & $\mathrm{U}$ & $\mathrm{O}$ & City of Electra & 108 N. Main St., Electra, TX 76360 & -- & -- & -- \\
\hline 80 & $1,048,480$ & 675,819 & 27,690 & 115,347 & $\mathrm{C}$ & $\mathrm{O}$ & Sabine River Authority & P.O. Box 579, Orange, TX 77631 & $\mathrm{X}$ & $\begin{array}{l}\text { Op. Mgr., } \\
\text { Donnie } \\
\text { Henson; } \\
\text { DA; SA }\end{array}$ & -- \\
\hline 81 & 220,100 & 37,100 & -- & 284,000 & $\mathrm{U}$ & $\mathrm{FC}, \mathrm{O}$ & $\begin{array}{l}\text { U.S. Army Corps of } \\
\text { Engineers }\end{array}$ & $\begin{array}{l}\text { P.O. Box 17300, Fort Worth, TX } \\
\quad 76102\end{array}$ & -- & -- & -- \\
\hline 82 & 26,000 & 12,720 & 895 & 71,100 & $\mathrm{U}$ & $\mathrm{O}$ & City of Gilmer & P.O. Box 760, Gilmer, TX 75644 & $\mathrm{X}$ & $\begin{array}{r}\text { Owner, Scott } \\
\text { Thompson }\end{array}$ & -- \\
\hline 83 & 14,391 & 6,950 & -- & 49,656 & $\mathrm{U}$ & $\mathrm{O}$ & City of Gladewater & $\begin{array}{l}\text { P.O. Box 551, Gladewater, TX } \\
75647\end{array}$ & -- & -- & -- \\
\hline
\end{tabular}


Table 3. Texas Natural Resource Conservation Commission permitted dams with maximum capacities in excess

\begin{tabular}{|c|c|c|c|c|c|c|c|c|c|}
\hline $\begin{array}{l}\text { Seq. } \\
\text { no. }\end{array}$ & $\begin{array}{c}\text { TNRCC } \\
\text { no. } \\
\text { (pl. 2) }\end{array}$ & $\begin{array}{l}\text { River } \\
\text { basin }\end{array}$ & $\begin{array}{c}\text { Stream } \\
\text { name }\end{array}$ & County & $\begin{array}{c}\text { Reservoir } \\
\text { name }\end{array}$ & Latitude & Longitude & $\begin{array}{c}\text { Year } \\
\text { com- } \\
\text { pleted }\end{array}$ & $\begin{array}{c}\text { Drain- } \\
\text { age } \\
\text { area } \\
\left(\mathrm{mi}^{2}\right)\end{array}$ \\
\hline 84 & TX03945 & Brazos & Salt Creek & Young & Lake Graham & $33^{\circ} 08^{\prime} 00^{\prime \prime}$ & $98^{\circ} 36^{\prime} 48^{\prime \prime}$ & 1958 & -- \\
\hline 85 & TX03956 & Brazos & Brazos River & Hood & Lake Granbury & $32^{\circ} 22^{\prime} 24^{\prime \prime}$ & $97^{\circ} 41^{\prime} 18^{\prime \prime}$ & 1969 & 16,113 \\
\hline 86 & TX00005 & Trinity & Denton Creek & Tarrant & Lake Grapevine & $32^{\circ} 58^{\prime} 00^{\prime \prime}$ & $97^{\circ} 03^{\prime} 00^{\prime \prime}$ & 1952 & -- \\
\hline 87 & TX02568 & Trinity & Elm Creek & Navarro & Lake Halbert & $32^{\circ} 04^{\prime} 36^{\prime \prime}$ & $96^{\circ} 24^{\prime} 12^{\prime \prime}$ & 1921 & -- \\
\hline 88 & TX00920 & Sabine & Little Sandy Creek & Wood & Lake Hawkins & $32^{\circ} 36^{\prime} 42^{\prime \prime}$ & $95^{\circ} 15^{\prime} 06^{\prime \prime}$ & 1962 & -- \\
\hline 89 & TX00936 & Sabine & Keyes Creek & Wood & Lake Holbrook & $32^{\circ} 41^{\prime} 06^{\prime \prime}$ & $95^{\circ} 33^{\prime} 06^{\prime \prime}$ & 1962 & -- \\
\hline 90 & TX03416 & San Jacinto & San Jacinto River & Harris & Lake Houston & $29^{\circ} 55^{\prime} 12^{\prime \prime}$ & $95^{\circ} 07^{\prime} 54^{\prime \prime}$ & 1954 & 2,828 \\
\hline 91 & TX04138 & Colorado & Colorado River & Scurry & Lake J.B. Thomas & $32^{\circ} 35^{\prime} 00^{\prime \prime}$ & $101^{\circ} 08^{\prime} 06^{\prime \prime}$ & 1952 & -- \\
\hline 92 & TX00583 & Neches & Gum Creek & Cherokee & Lake Jacksonville & $31^{\circ} 54^{\prime} 30^{\prime \prime}$ & $95^{\circ} 18^{\prime} 30^{\prime \prime}$ & 1957 & 41 \\
\hline 93 & TX04358 & Red & Big Wichita River & Baylor & Lake Kemp & $33^{\circ} 45^{\prime} 18^{\prime \prime}$ & $99^{\circ} 08^{\prime} 42^{\prime \prime}$ & 1923 & 2,086 \\
\hline
\end{tabular}

\begin{tabular}{|c|c|c|c|c|c|c|c|c|c|}
\hline 94 & TX01010 & Red & North Fork Little Wichita River & Archer & Lake Kickapoo & $33^{\circ} 39^{\prime} 48^{\prime \prime}$ & $98^{\circ} 46^{\prime} 42^{\prime \prime}$ & 1945 & .43 \\
\hline 95 & TX00536 & Trinity & Indina Creek & Cooke & Lake Kiowa & $33^{\circ} 33^{\prime} 12^{\prime \prime}$ & $97^{\circ} 00^{\prime} 42^{\prime \prime}$ & 1970 & -- \\
\hline 96 & TX02703 & Brazos & Cedar Creek & Taylor & Lake Kirby & $32^{\circ} 23^{\prime} 06^{\prime \prime}$ & $99^{\circ} 43^{\prime} 42^{\prime \prime}$ & 1928 & 41.6 \\
\hline 97 & TX00976 & Neches & Trib._-Angelina River & Angelina & Lake Kurth & $31^{\circ} 27^{\prime} 06^{\prime \prime}$ & $94^{\circ} 42^{\prime} 00^{\prime \prime}$ & 1960 & 3.9 \\
\hline 98 & TX01417 & Brazos & Leon River & Eastland & Lake Leon & $32^{\circ} 21^{\prime} 36^{\prime \prime}$ & $98^{\circ} 40^{\prime} 30^{\prime \prime}$ & 1954 & 252 \\
\hline 99 & TX00008 & Trinity & Elm Fork Trinity River & Denton & Lake Lewisville & $33^{\circ} 04^{\prime} 00^{\prime \prime}$ & $96^{\circ} 57^{\prime} 48^{\prime \prime}$ & 1954 & -- \\
\hline 100 & TX04455 & Brazos & Navasota River & Robertson & Lake Limestone & $31^{\circ} 19^{\prime} 30^{\prime \prime}$ & $96^{\circ} 19^{\prime} 12^{\prime \prime}$ & 1978 & 674 \\
\hline 101 & TX00986 & Colorado & Colorado River & Burnet & Lake Lyndon B. Johnson & $30^{\circ} 33^{\prime} 18^{\prime \prime}$ & $98^{\circ} 20^{\prime} 18^{\prime \prime}$ & 1951 & 37.82 \\
\hline 102 & TX00023 & Canadian & Canadian River & Hutchinson & Lake Meredith & $35^{\circ} 43^{\prime} 00^{\prime \prime}$ & $101^{\circ} 33^{\prime} 12^{\prime \prime}$ & 1965 & -- \\
\hline 103 & TX01061 & Brazos & Navasota River & Limestone & Lake Mexia & $31^{\circ} 38^{\prime} 36^{\prime \prime}$ & $96^{\circ} 34^{\prime} 42^{\prime \prime}$ & 1961 & 198 \\
\hline 104 & TX01225 & Brazos & Rock Creek & Parker & Lake Mineral Wells & $32^{\circ} 49^{\prime} 00^{\prime \prime}$ & $98^{\circ} 02^{\prime} 30^{\prime \prime}$ & 1920 & 63 \\
\hline 105 & TX04585 & Neches & Bayou Loco & Nacogdoches & Lake Nacogdoches & $31^{\circ} 35^{\prime} 18^{\prime \prime}$ & $94^{\circ} 49^{\prime} 36^{\prime \prime}$ & 1977 & -- \\
\hline 106 & TX03139 & Colorado & South Concho River & Tom Green & Lake Nasworthy & $31^{\circ} 23^{\prime} 18^{\prime \prime}$ & $100^{\circ} 28^{\prime} 42^{\prime \prime}$ & 1930 & 3,833 \\
\hline 107 & TX00775 & Red & Farmers Creek & Montague & Lake Nocona & $33^{\circ} 53^{\prime} 00^{\prime \prime}$ & $97^{\circ} 39^{\prime} 06^{\prime \prime}$ & 1960 & 94 \\
\hline 108 & TX00020 & Cypress & Cypress Creek & Marion & Lake O' the Pines & $32^{\circ} 45^{\prime} 54^{\prime \prime}$ & $94^{\circ} 29^{\prime} 48^{\prime \prime}$ & 1959 & -- \\
\hline 109 & TX01407 & Brazos & Leon River & Eastland & Lake Olden & $32^{\circ} 22^{\prime} 18^{\prime \prime}$ & $98^{\circ} 46^{\prime} 12^{\prime \prime}$ & 1920 & 207 \\
\hline 110 & TX00170 & Neches & Neches River & Anderson & Lake Palestine & $32^{\circ} 03^{\prime} 18^{\prime \prime}$ & $95^{\circ} 26^{\prime} 18^{\prime \prime}$ & 1971 & -- \\
\hline 111 & TX03845 & Brazos & Palo Pinto Creek & Palo Pinto & Lake Palo Pinto & $32^{\circ} 38^{\prime} 48^{\prime \prime}$ & $98^{\circ} 16^{\prime} 06^{\prime \prime}$ & 1964 & 471 \\
\hline
\end{tabular}




\begin{tabular}{|c|c|c|c|c|c|c|c|c|c|c|c|}
\hline $\begin{array}{l}\text { Seq. } \\
\text { no. }\end{array}$ & $\begin{array}{c}\text { Maximum } \\
\text { capacity } \\
\text { (acre-ft) }\end{array}$ & $\begin{array}{l}\text { Normal } \\
\text { capacity } \\
\text { (acre-ft) }\end{array}$ & $\begin{array}{c}\text { Surface } \\
\text { area } \\
\text { (acres) }\end{array}$ & $\begin{array}{c}\text { Maximum } \\
\text { discharge } \\
\left(\mathrm{ft}^{3} / \mathrm{s}\right)\end{array}$ & $\begin{array}{c}\text { Type } \\
\text { of } \\
\text { spill- } \\
\text { way }\end{array}$ & $\begin{array}{l}\text { Pur- } \\
\text { pose } \\
\text { of } \\
\text { dam }\end{array}$ & Owner name & Owner address & $\begin{array}{l}\text { Ques- } \\
\text { tion- } \\
\text { aire } \\
\text { returned }\end{array}$ & $\begin{array}{l}\text { Updated } \\
\text { infor- } \\
\text { mation }\end{array}$ & $\begin{array}{l}\text { Outflow peak- } \\
\text { streamflow } \\
\text { frequency } \\
\left(\mathrm{ft}^{3} / \mathrm{s}\right)\end{array}$ \\
\hline 84 & 105,000 & 39,000 & -- & -- & $\mathrm{U}$ & $\mathrm{O}$ & City of Graham & P.O. Box 1449, Graham, TX 76450 & -- & -- & -- \\
\hline 85 & 136,823 & 136,823 & 8,700 & 635,000 & $\mathrm{C}$ & $\mathrm{O}$ & Brazos River Authority & P.O. Box 7555, Waco, TX 76714 & $\mathrm{X}$ & $\begin{array}{l}\text { DA; norm. } \\
\text { cap.; max. } \\
\text { cap.; SA }\end{array}$ & -- \\
\hline 86 & 758,800 & 181,100 & -- & 182,500 & $\mathrm{U}$ & $\mathrm{FC}, \mathrm{O}$ & $\begin{array}{l}\text { U.S. Army Corps of } \\
\text { Engineers }\end{array}$ & $\begin{array}{l}\text { P.O. Box 17300, Fort Worth, TX } \\
\quad 76102\end{array}$ & -- & -- & -- \\
\hline 87 & 14,400 & 7,357 & -- & -- & $\mathrm{U}$ & $\mathrm{O}$ & City of Corsicana & $\begin{array}{l}200 \text { N. 12th St., Corsicana, TX } \\
75110\end{array}$ & -- & -- & -- \\
\hline 88 & 40,200 & 11,890 & -- & 29,259 & $\mathrm{U}$ & $\mathrm{FC}, \mathrm{O}$ & Wood County & $\begin{array}{l}\text { County Judge, Wood County } \\
\quad \text { Courthouse, Quitman, TX } 75763\end{array}$ & -- & -- & -- \\
\hline 89 & 15,980 & 7,990 & -- & -- & $\mathrm{U}$ & $\mathrm{FC}, \mathrm{O}$ & Wood County & $\begin{array}{l}\text { County Judge, Wood County } \\
\text { Courthouse, Quitman, TX } 75763\end{array}$ & -- & -- & -- \\
\hline 90 & 281,800 & 146,700 & 12,240 & 840,000 & $\mathrm{C}$ & $\mathrm{O}$ & City of Houston & $\begin{array}{l}\text { Public Works Dept., P.O. Box 1562, } \\
\text { Houston, TX } 77251\end{array}$ & -- & -- & -- \\
\hline 91 & 360,000 & 204,000 & -- & 161,000 & $\mathrm{U}$ & $\mathrm{O}$ & Colorado River MUD & P.O. Box 869, Big Spring, TX 79721 & -- & -- & -- \\
\hline 92 & 56,083 & 30,500 & 1,325 & 26,588 & $\mathrm{U}$ & $\mathrm{O}$ & City of Jacksonville & $\begin{array}{l}\text { P.O. Box 1390, Jacksonville, TX } \\
75766\end{array}$ & $\mathrm{X}$ & -- & -- \\
\hline 93 & $1,097,200$ & 268,100 & 15,590 & 527,500 & $\mathrm{U}$ & $\mathrm{FC}, \mathrm{O}$ & $\begin{array}{l}\text { U.S. Army Corps of } \\
\text { Engineers }\end{array}$ & P.O. Box 61, Tulsa, OK 74121 & $\mathrm{X}$ & All statistics & $\begin{array}{l}\mathrm{Q}_{2}=5,830 \\
\mathrm{Q}_{5}=5,830 \\
\mathrm{Q}_{10}=5,830 \\
\mathrm{Q}_{25}=5,830 \\
\mathrm{Q}_{50}=5,830 \\
\mathrm{Q}_{100}=7,100\end{array}$ \\
\hline 94 & 202,000 & 106,000 & 6,200 & 82,500 & $\mathrm{U}$ & $\mathrm{O}$ & City of Wichita Falls & $\begin{array}{l}\text { P.O. Box 1431, Wichita Falls, TX } \\
76307\end{array}$ & -- & -- & -- \\
\hline 95 & 23,520 & 7,000 & -- & 12,400 & $\mathrm{U}$ & $\mathrm{O}$ & $\begin{array}{l}\text { Lake Kiowa Property } \\
\text { Owners Association }\end{array}$ & $\begin{array}{l}905 \text { Kiowa Dr. W., Lake Kiowa, TX } \\
76240\end{array}$ & -- & -- & -- \\
\hline 96 & 17,811 & 7,620 & 780 & 104,000 & $\mathrm{U}$ & $\mathrm{O}$ & City of Abilene & P.O. Box 60, Abilene, TX 79604 & $\mathrm{X}$ & No info. & -- \\
\hline 97 & 27,360 & 16,200 & 800 & 118 & $\mathrm{U}$ & $\mathrm{O}$ & Champion Paper Co. & $\begin{array}{l}\text { Power and Utilities, P.O. Box 149, } \\
\text { Lufkin, TX } 75901\end{array}$ & $\mathrm{X}$ & SA & -- \\
\hline 98 & 72,250 & 27,290 & 1,590 & 230,367 & $\mathrm{U}$ & $\mathrm{O}$ & Eastland County WSD & Rt. 1, Box 157, Ranger, TX 76470 & -- & -- & -- \\
\hline 99 & $2,082,800$ & 464,500 & -- & 216,800 & $\mathrm{U}$ & $\mathrm{FC}, \mathrm{O}$ & $\begin{array}{l}\text { U.S. Army Corps of } \\
\text { Engineers }\end{array}$ & $\begin{array}{l}\text { P.O. Box 17300, Fort Worth, TX } \\
\quad 76102\end{array}$ & -- & -- & -- \\
\hline 100 & 333,048 & 215,751 & 13,379 & 150,725 & $\mathrm{U}$ & $\mathrm{O}$ & Brazos River Authority & P.O. Box 7555, Waco, TX 76714 & $\mathrm{X}$ & $\begin{array}{l}\text { Norm. cap.; } \\
\text { max. cap.; } \\
\text { SA; max. } \\
\text { dis. }\end{array}$ & -- \\
\hline 101 & 227,000 & 138,000 & 6,375 & $1,633,409$ & $\mathrm{C}$ & $\mathrm{O}$ & $\begin{array}{l}\text { Lower Colorado River } \\
\text { Authority }\end{array}$ & P.O. Box 220, Austin, TX 78767 & $\mathrm{X}$ & -- & -- \\
\hline 102 & $2,434,215$ & 864,400 & -- & 61,100 & $\mathrm{U}$ & $\mathrm{FC}, \mathrm{O}$ & Canadian River MWA & P.O. Box 99, Sanford, TX 79078 & -- & -- & -- \\
\hline 103 & 45,000 & 10,000 & -- & 161,456 & $\mathrm{U}$ & $\mathrm{O}$ & Bistone MWSD & P.O. Box 145, Mexia, TX 76667 & -- & -- & -- \\
\hline 104 & 16,356 & 7,065 & 640 & 122,427 & $\mathrm{U}$ & $\mathrm{O}$ & City of Mineral Wells & $\begin{array}{l}\text { P.O. Box 339, Mineral Wells, TX } \\
\quad 76068\end{array}$ & $\mathrm{X}$ & DA; SA & -- \\
\hline 105 & 122,000 & 42,318 & -- & 50,160 & $\mathrm{U}$ & $\mathrm{O}$ & City of Nacogdoches & $\begin{array}{l}\text { P.O. Box 630648, Nacogdoches, TX } \\
\quad 75963\end{array}$ & -- & -- & -- \\
\hline 106 & 42,500 & 13,990 & 1,210 & 659,064 & $\mathrm{C}$ & $\mathrm{O}$ & City of San Angelo & $\begin{array}{l}\text { P.O. Box 1751, San Angelo, TX } \\
\quad 76902\end{array}$ & $\mathrm{X}$ & $\mathrm{DA} ; \mathrm{SA}$ & -- \\
\hline 107 & 59,688 & 25,389 & 1,470 & 115,597 & $\mathrm{U}$ & $\mathrm{O}$ & $\begin{array}{l}\text { North Montague County } \\
\text { WSD }\end{array}$ & 101 Cook St., Nocona, TX 76255 & $\mathrm{X}$ & $\begin{array}{l}\text { Phone; max. } \\
\text { dis.; dis. } \\
\text { freq. }\end{array}$ & $\begin{array}{l}\mathrm{Q}_{5}=4,539 \\
\mathrm{Q}_{10}=6,683 \\
\mathrm{Q}_{25}=9,420 \\
\mathrm{Q}_{50}=12,038 \\
\mathrm{Q}_{100}=15,000\end{array}$ \\
\hline 108 & $1,856,500$ & 254,900 & -- & 68,200 & $\mathrm{U}$ & $\mathrm{FC}, \mathrm{O}$ & $\begin{array}{l}\text { U.S. Army Corps of } \\
\text { Engineers }\end{array}$ & $\begin{array}{l}\text { P.O. Box 17300, Fort Worth, TX } \\
\quad 76102\end{array}$ & -- & -- & -- \\
\hline 109 & 21,700 & 1,607 & 130 & 241,379 & $\mathrm{C}$ & $\mathrm{O}$ & $\begin{array}{l}\text { Eastland Industrial } \\
\text { Foundation }\end{array}$ & 104 N. Lamar, Eastland, TX 76448 & -- & -- & -- \\
\hline 110 & $1,045,000$ & 411,840 & 25,500 & 187,056 & $\mathrm{U}$ & $\mathrm{O}$ & Upper Neches River MWA & P.O. Drawer Y, Palestine, TX 75801 & $\mathrm{X}$ & SA & -- \\
\hline 111 & 170,735 & 44,100 & 2,661 & 313,757 & $\mathrm{U}$ & $\mathrm{O}$ & $\begin{array}{l}\text { Palo Pinto County MWD } \\
\text { No. } 1\end{array}$ & $\begin{array}{l}\text { P.O. Box 387, Mineral Wells, TX } \\
\quad 76067\end{array}$ & -- & -- & -- \\
\hline
\end{tabular}


Table 3. Texas Natural Resource Conservation Commission permitted dams with maximum capacities in excess

\begin{tabular}{|c|c|c|c|c|c|c|c|c|c|}
\hline $\begin{array}{l}\text { Seq. } \\
\text { no. }\end{array}$ & $\begin{array}{c}\text { TNRCC } \\
\text { no. } \\
\text { (pl. 2) }\end{array}$ & $\begin{array}{l}\text { River } \\
\text { basin }\end{array}$ & $\begin{array}{c}\text { Stream } \\
\text { name }\end{array}$ & County & $\begin{array}{c}\text { Reservoir } \\
\text { name }\end{array}$ & Latitude & Longitude & $\begin{array}{c}\text { Year } \\
\text { com- } \\
\text { pleted }\end{array}$ & $\begin{array}{c}\text { Drain- } \\
\text { age } \\
\text { area } \\
\left(\mathrm{mi}^{2}\right)\end{array}$ \\
\hline
\end{tabular}

\begin{tabular}{|c|c|c|c|c|c|c|c|c|c|}
\hline 113 & TX03814 & Red & Wanderers Creek & Hardeman & Lake Pauline & $34^{\circ} 15^{\prime} 00^{\prime \prime}$ & $99^{\circ} 40^{\prime} 18^{\prime \prime}$ & 1905 & 42.6 \\
\hline 114 & TX00950 & Sabine & Dry Creek & Wood & Lake Quitman & $32^{\circ} 51^{\prime} 30^{\prime \prime}$ & $95^{\circ} 27^{\prime} 00^{\prime \prime}$ & 1962 & -- \\
\hline 115 & TX00837 & Trinity & East Fork Trinity River & Kaufman & Lake Ray Hubbard & $32^{\circ} 48^{\prime} 06^{\prime \prime}$ & $96^{\circ} 30^{\prime} 24^{\prime \prime}$ & 1969 & -- \\
\hline 116 & TX00013 & Brazos & Yegua Creek & Burleson & Lake Somerville & $30^{\circ} 20^{\prime} 00^{\prime \prime}$ & $96^{\circ} 32^{\prime} 00^{\prime \prime}$ & 1967 & 1.57 \\
\hline 117 & TX03778 & Brazos & Paint Creek & Haskell & Lake Stamford & $33^{\circ} 04^{\prime} 18^{\prime \prime}$ & $99^{\circ} 33^{\prime} 36^{\prime \prime}$ & 1953 & -- \\
\hline 118 & TX03549 & Neches & Striker Creek & Rusk & Lake Striker & $31^{\circ} 56^{\prime} 06^{\prime \prime}$ & $94^{\circ} 58^{\prime} 30^{\prime \prime}$ & 1956 & -- \\
\hline 119 & TX04356 & Sulphur & White Oak Creek & Hopkins & Lake Sulphur Springs & $33^{\circ} 10^{\prime} 24^{\prime \prime}$ & $95^{\circ} 36^{\prime} 36^{\prime \prime}$ & 1973 & -- \\
\hline 120 & TX02735 & Brazos & Bitter Creek & Nolan & Lake Sweetwater & $32^{\circ} 26^{\prime} 18^{\prime \prime}$ & $100^{\circ} 18^{\prime} 06^{\prime \prime}$ & 1930 & 104 \\
\hline 121 & TX03587 & Red & $\begin{array}{l}\text { Prairie Dog Town Fork Red } \\
\text { River }\end{array}$ & Randall & Lake Tanglewood & $35^{\circ} 02^{\prime} 24^{\prime \prime}$ & $101^{\circ} 46^{\prime} 12^{\prime \prime}$ & 1965 & 832.7 \\
\hline 122 & TX00491 & Sabine & Sabine River & Rains & Lake Tawakoni & $32^{\circ} 48^{\prime} 42^{\prime \prime}$ & $95^{\circ} 55^{\prime} 00^{\prime \prime}$ & 1960 & 752 \\
\hline 123 & TX04779 & Lavaca & Navidad River & Jackson & Lake Texana & $28^{\circ} 53^{\prime} 24^{\prime \prime}$ & $96^{\circ} 34^{\prime} 42^{\prime \prime}$ & 1981 & 1,392 \\
\hline 124 & OK10317 & Red & Red River & Grayson & Lake Texoma & $33^{\circ} 49^{\prime} 06^{\prime \prime}$ & $96^{\circ} 34^{\prime} 12^{\prime \prime}$ & 1944 & 39,719 \\
\hline 125 & TX00315 & Neches & Blackwater Creek & Shelby & Lake Timpson & $31^{\circ} 50^{\prime} 42^{\prime \prime}$ & $94^{\circ} 25^{\prime} 48^{\prime \prime}$ & 1956 & -- \\
\hline 126 & TX01087 & Colorado & Colorado River & Travis & Lake Travis & $30^{\circ} 23^{\prime} 30^{\prime \prime}$ & $97^{\circ} 54^{\prime} 24^{\prime \prime}$ & 1942 & -- \\
\hline 127 & TX00245 & Neches & Prairie Creek & Smith & Lake Tyler & $32^{\circ} 12^{\prime} 42^{\prime \prime}$ & $95^{\circ} 10^{\prime} 18^{\prime \prime}$ & 1949 & 42 \\
\hline 128 & TX00244 & Neches & Mud Creek & Smith & Lake Tyler East & $32^{\circ} 12^{\prime} 36^{\prime \prime}$ & $95^{\circ} 08^{\prime} 42^{\prime \prime}$ & 1967 & 65 \\
\hline 129 & TX00016 & Brazos & Bosque River & McLennan & Lake Waco & $31^{\circ} 36^{\prime} 00^{\prime \prime}$ & $97^{\circ} 13^{\prime} 00^{\prime \prime}$ & 1965 & -- \\
\hline 130 & TX01089 & Colorado & Decker Creek & Travis & Lake Walter E. Long & $30^{\circ} 17^{\prime} 06^{\prime \prime}$ & $97^{\circ} 35^{\prime} 48^{\prime \prime}$ & 1967 & 9.2 \\
\hline 131 & TX01255 & Trinity & South Prong Waxahachie Creek & Ellis & Lake Waxahachie & $32^{\circ} 20^{\prime} 30^{\prime \prime}$ & $96^{\circ} 48^{\prime} 18^{\prime \prime}$ & 1956 & 31,000 \\
\hline 132 & TX01222 & Trinity & Clear Fork Trinity River & Parker & Lake Weatherford & $32^{\circ} 46^{\prime} 18^{\prime \prime}$ & $97^{\circ} 40^{\prime} 30^{\prime \prime}$ & 1957 & 109 \\
\hline 133 & TX00017 & Brazos & Brazos River & Bosque & Lake Whitney & $31^{\circ} 52^{\prime} 00^{\prime \prime}$ & $97^{\circ} 22^{\prime} 12^{\prime \prime}$ & 1951 & 27.59 \\
\hline 134 & TX01017 & Red & Holliday Creek & Wichita & Lake Wichita & $33^{\circ} 50^{\prime} 42^{\prime \prime}$ & $98^{\circ} 32^{\prime} 18^{\prime \prime}$ & 1901 & 143 \\
\hline 135 & TX00940 & Sabine & Big Sandy Creek & Wood & Lake Winnsboro & $32^{\circ} 53^{\prime} 12^{\prime \prime}$ & $95^{\circ} 20^{\prime} 42^{\prime \prime}$ & 1962 & -- \\
\hline 136 & TX00785 & Trinity & West Fork Trinity River & Tarrant & Lake Worth & $32^{\circ} 47^{\prime} 30^{\prime \prime}$ & $97^{\circ} 24^{\prime} 54^{\prime \prime}$ & 1914 & 2,064 \\
\hline 137 & TX00007 & Trinity & East Fork Trinity River & Collin & Lavon Reservoir & $33^{\circ} 02^{\prime} 00^{\prime \prime}$ & $96^{\circ} 29^{\prime} 00^{\prime \prime}$ & 1953 & -- \\
\hline 138 & TX00119 & San Jacinto & Lewis Creek & Montgomery & Lewis Creek Reservoir & $30^{\circ} 25^{\prime} 48^{\prime \prime}$ & $95^{\circ} 32^{\prime} 36^{\prime \prime}$ & 1969 & -- \\
\hline
\end{tabular}




\begin{tabular}{|c|c|c|c|c|c|c|c|c|c|c|c|}
\hline $\begin{array}{l}\text { Seq. } \\
\text { no. }\end{array}$ & $\begin{array}{l}\text { Maximum } \\
\text { capacity } \\
\text { (acre-ft) }\end{array}$ & $\begin{array}{l}\text { Normal } \\
\text { capacity } \\
\text { (acre-ft) }\end{array}$ & $\begin{array}{l}\text { Surface } \\
\text { area } \\
\text { (acres) }\end{array}$ & $\begin{array}{l}\text { Maximum } \\
\text { discharge } \\
\left(\mathrm{ft}^{3} / \mathrm{s}\right)\end{array}$ & $\begin{array}{l}\text { Type } \\
\text { of } \\
\text { spill- } \\
\text { way }\end{array}$ & $\begin{array}{l}\text { Pur- } \\
\text { pose } \\
\text { of } \\
\text { dam }\end{array}$ & Owner name & Owner address & $\begin{array}{l}\text { Ques- } \\
\text { tion- } \\
\text { aire } \\
\text { returned }\end{array}$ & $\begin{array}{l}\text { Updated } \\
\text { infor- } \\
\text { mation }\end{array}$ & $\begin{array}{l}\text { Outflow peak- } \\
\text { streamflow } \\
\text { frequency } \\
\left(\mathrm{ft}^{3} / \mathrm{s}\right)\end{array}$ \\
\hline 112 & 667,00 & 25,560 & 1,550 & 99,580 & $\mathrm{U}$ & $\mathrm{O}$ & City of Cleburne & P.O. Box 657, Cleburne, TX 76033 & $\mathrm{X}$ & $\begin{array}{l}\text { DA; norm. } \\
\text { cap.; SA; } \\
\text { dis. freq. }\end{array}$ & $\begin{array}{l}\mathrm{Q}_{2}=4,000 \\
\mathrm{Q}_{5}=9,000 \\
\mathrm{Q}_{10}=13,500 \\
\mathrm{Q}_{25}=20,750 \\
\mathrm{Q}_{50}=26,750\end{array}$ \\
\hline 113 & 16,000 & 7,000 & 609 & -- & $\mathrm{U}$ & $\mathrm{O}$ & West Texas Utilities Co. & P.O. Box 841, Abilene, TX 79604 & $\mathrm{X}$ & -- & -- \\
\hline 114 & 29,200 & 7,440 & -- & 34,400 & $\mathrm{U}$ & $\mathrm{FC}, \mathrm{O}$ & Wood County & $\begin{array}{l}\text { County Judge, Wood County } \\
\text { Courthouse, Quitman, TX } 75763\end{array}$ & -- & -- & -- \\
\hline 115 & 583,600 & 490,000 & -- & 357,112 & $\mathrm{C}$ & $\mathrm{O}$ & City of Dallas & City Hall, Dallas, TX 75201 & -- & -- & -- \\
\hline 116 & $1,028,800$ & 160,100 & 11,460 & 286,000 & $\mathrm{U}$ & $\mathrm{FC}, \mathrm{O}$ & $\begin{array}{l}\text { U.S. Army Corps of } \\
\text { Engineers }\end{array}$ & $\begin{array}{l}\text { P.O. Box 17300, Fort Worth, TX } \\
\quad 76102\end{array}$ & -- & -- & -- \\
\hline 117 & 150,000 & 57,927 & -- & 134,500 & $\mathrm{U}$ & $\mathrm{O}$ & City of Stamford & $\begin{array}{l}\text { P.O. Drawer 191, Stamford, TX } \\
79553\end{array}$ & -- & -- & -- \\
\hline 118 & 80,000 & 26,960 & -- & 130,000 & -- & $\mathrm{O}$ & $\begin{array}{l}\text { Angelina-Nacogdoches } \\
\text { County WCID }\end{array}$ & Rt. 1, Box 75, Reklaw, TX 75784 & -- & -- & -- \\
\hline 119 & 34,700 & 17,838 & -- & 49,800 & $\mathrm{U}$ & $\mathrm{O}$ & City of Sulphur Springs & $\begin{array}{l}125 \text { S. Davis St., Sulphur Springs, } \\
\text { TX } 75482\end{array}$ & -- & -- & -- \\
\hline 120 & 19,340 & 2,544 & 220.5 & 208,954 & $\mathrm{U}$ & $\mathrm{O}$ & City of Sweetwater & $\begin{array}{l}\text { P.O. Box 450, Sweetwater, TX } \\
\quad 79556\end{array}$ & -- & -- & -- \\
\hline 121 & 13,275 & 4,897 & 258 & 194,426 & $\mathrm{U}$ & $\mathrm{FC}, \mathrm{O}$ & Lake Tanglewood Inc. & $\begin{array}{l}\text { Rt. 8, Box 1000, Amarillo, TX } \\
\quad 79118\end{array}$ & $\mathrm{X}$ & -- & -- \\
\hline 122 & $1,660,000$ & 927,440 & 36,700 & 131,500 & $\mathrm{U}$ & $\mathrm{O}$ & Sabine River Authority & P.O. Box 579, Orange, TX 77631 & $\mathrm{x}$ & $\begin{array}{l}\text { Op. Mgr., } \\
\text { Donnie } \\
\text { Henson; } \\
\text { DA; norm. } \\
\text { cap.; SA }\end{array}$ & -- \\
\hline 123 & 197,099 & 163,506 & 10,134 & 176,000 & $\mathrm{C}$ & $\mathrm{O}$ & $\begin{array}{l}\text { Lavaca-Navidad River } \\
\quad \text { Authority }\end{array}$ & P.O. Box 429, Edna, TX 77957 & $\mathrm{X}$ & $\begin{array}{l}\text { DA; norm } \\
\text { cap.; max. } \\
\text { cap.; SA; } \\
\text { max. dis. }\end{array}$ & -- \\
\hline 124 & $8,600,000$ & $2,580,400$ & 86,910 & $1,125,000$ & $\mathrm{U}$ & $\mathrm{FC}, \mathrm{O}$ & $\begin{array}{l}\text { U.S. Army Corps of } \\
\text { Engineers }\end{array}$ & P.O. Box 61, Tulsa, OK 74121 & $\mathrm{X}$ & $\begin{array}{l}\text { All but max. } \\
\text { cap. }\end{array}$ & $\begin{array}{l}\mathrm{Q}_{2}=40,000 \\
\mathrm{Q}_{5}=45,000 \\
\mathrm{Q}_{10}=50,000 \\
\mathrm{Q}_{25}=60,000 \\
\mathrm{Q}_{50}=121,550 \\
\mathrm{Q}_{100}=175,000\end{array}$ \\
\hline 125 & 60,148 & 1,881 & 250 & 12,773 & $\mathrm{U}$ & $\mathrm{O}$ & Shelby County FWSD No. 1 & P.O. Box 106, Timpson, TX 75975 & $\mathrm{X}$ & SA & -- \\
\hline 126 & $3,223,000$ & $1,172,600$ & 18,929 & 572,000 & $\mathrm{U}$ & $\mathrm{FC}, \mathrm{O}$ & $\begin{array}{l}\text { Lower Colorado River } \\
\text { Authority }\end{array}$ & P.O. Box 220, Austin, TX 78767 & $\mathrm{x}$ & -- & -- \\
\hline 127 & 85,810 & 42,500 & 2,365 & 32,666 & $\mathrm{U}$ & $\mathrm{O}$ & City of Tyler & P.O. Box 2039, Tyler, TX 75710 & $\mathrm{X}$ & DA; SA; ph. & -- \\
\hline 128 & 85,010 & 43,000 & 2,507 & 31,000 & $\mathrm{U}$ & $\mathrm{O}$ & City of Tyler & P.O. Box 2039, Tyler, TX 75710 & $\mathrm{X}$ & $\mathrm{DA} ; \mathrm{SA} ; \mathrm{ph}$. & -- \\
\hline 129 & 828,300 & 152,500 & -- & 563,300 & $\mathrm{C}$ & $\mathrm{FC}, \mathrm{O}$ & $\begin{array}{l}\text { U.S. Army Corps of } \\
\text { Engineers }\end{array}$ & $\begin{array}{l}\text { P.O. Box 17300, Fort Worth, TX } \\
76102\end{array}$ & -- & -- & -- \\
\hline 130 & 45,200 & 33,940 & 1,280 & 34,467 & $\mathrm{C}$ & $\mathrm{O}$ & City of Austin & P.O. Box 1088, Austin, TX 78767 & $\mathrm{X}$ & $\mathrm{DA} ; \mathrm{SA}$ & -- \\
\hline 131 & 23,663 & 13,500 & 690 & 56,521 & $\mathrm{U}$ & $\mathrm{O}$ & Ellis County WCID No. 1 & $\begin{array}{l}\text { P.O. Box 757, Waxahachie, TX } \\
\quad 75165\end{array}$ & -- & -- & -- \\
\hline 132 & 37,520 & 19,866 & 1,090 & 68,800 & $\mathrm{U}$ & $\mathrm{O}$ & City of Weatherford & $\begin{array}{l}\text { P.O. Box 255, Weatherford, TX } \\
76086\end{array}$ & $\mathrm{X}$ & -- & -- \\
\hline 133 & $2,100,400$ & 627,100 & 23,560 & 684,000 & $\mathrm{C}$ & $\mathrm{FC}, \mathrm{O}$ & $\begin{array}{l}\text { U.S. Army Corps of } \\
\text { Engineers }\end{array}$ & $\begin{array}{l}\text { P.O. Box 17300, Fort Worth, TX } \\
76102\end{array}$ & -- & -- & -- \\
\hline 134 & 57,280 & 5620 & 1,310 & 76,300 & $\mathrm{U}$ & $\mathrm{FC}, \mathrm{O}$ & $\begin{array}{l}\text { Wichita County WCID } \\
\text { No. } 2 \text { and City of Wichita } \\
\text { Falls }\end{array}$ & $\begin{array}{l}402 \text { E. Scott St., Wichita Falls, TX } \\
76301\end{array}$ & -- & -- & -- \\
\hline 135 & 30,500 & 8100 & -- & -- & $\mathrm{U}$ & $\mathrm{FC}, \mathrm{O}$ & Wood County & $\begin{array}{l}\text { County Judge, Wood County } \\
\text { Courthouse, Quitman, TX } 75763\end{array}$ & -- & -- & -- \\
\hline 136 & 96,225 & 38,130 & 3,560 & 101,760 & $\mathrm{U}$ & $\mathrm{O}$ & City of Fort Worth & $\begin{array}{l}1000 \text { Throckmorton St., Fort Worth, } \\
\text { TX } 76102\end{array}$ & -- & -- & -- \\
\hline 137 & 921,200 & 456,500 & -- & 357,700 & $\mathrm{C}$ & $\mathrm{FC}, \mathrm{O}$ & $\begin{array}{l}\text { U.S. Army Corps of } \\
\text { Engineers }\end{array}$ & $\begin{array}{l}\text { P.O. Box 17300, Fort Worth, TX } \\
\quad 76102\end{array}$ & -- & -- & -- \\
\hline 138 & 18,586 & 16,600 & -- & 11,232 & $\mathrm{C}$ & $\mathrm{O}$ & Gulf States Utilities Co. & P.O. Box 435, Willis, TX 77379 & -- & -- & -- \\
\hline
\end{tabular}


Table 3. Texas Natural Resource Conservation Commission permitted dams with maximum capacities in excess

\begin{tabular}{cccccccc}
\hline $\begin{array}{c}\text { Seq. } \\
\text { no. }\end{array}$ & $\begin{array}{c}\text { TNRCC } \\
\text { no. } \\
\text { (pl. 2) }\end{array}$ & $\begin{array}{c}\text { River } \\
\text { basin }\end{array}$ & $\begin{array}{c}\text { Stream } \\
\text { name }\end{array}$ & County & & $\begin{array}{c}\text { Reservoir } \\
\text { name }\end{array}$ & $\begin{array}{c}\text { Year } \\
\text { age } \\
\text { area } \\
\left(\mathbf{m i}^{2}\right)\end{array}$ \\
\hline 139 & TX03823 & Trinity & Trinity River & San Jacinto & Livingston Reservoir & $\begin{array}{c}\text { Latitude } \\
\text { pleted }\end{array}$ & $\begin{array}{c}\text { Longitude } \\
16,583\end{array}$
\end{tabular}

Baylor

Millers Creek Reservoir $33^{\circ} 25^{\prime} 24^{\prime \prime} \quad 99^{\circ} 22^{\prime} 06^{\prime \prime} \quad 1974 \quad 241$

$\begin{array}{lll}145 & \text { TX06420 } & \text { Colorado } \\ 146 & \text { TX04013 } & \text { Cypress } \\ 147 & \text { TX00827 } & \text { Trinity } \\ & & \\ 148 & \text { TX00330 } & \text { Sabine }\end{array}$

Trib.-Beals Creek

Mitchell

Mitchell County Reservoir

32॰14'24" $101^{\circ} 06^{\prime} 18^{\prime \prime} \quad 1991$

Dallas

Mountain Creek Lake

Murvaul Bayou

Panola

Murvaul Bayou Reservoir

$33^{\circ} 04^{\prime} 48^{\prime \prime} \quad 95^{\circ} 02^{\prime} 36^{\prime \prime} \quad 1973$

$32^{\circ} 43^{\prime} 54^{\prime \prime} \quad 96^{\circ} 56^{\prime} 36^{\prime \prime} \quad 1937 \quad 295$

$32^{\circ} 02^{\prime} 00^{\prime \prime} \quad 94^{\circ} 25^{\prime} 12^{\prime \prime} \quad 1956$

Sulphur Springs Draw

Howard

Natural Dam Lake

Richland Creek

Navarro

Navarro Mills Lake

North Fork Buffalo Creek

Wichita

North Fork Buffalo Creek Reservoir

South Fork Grapevine Creek

Dallas

North Lake

Concho River

Tom Green

O.C. Fisher Lake

Coke

Oak Creek Reservoir

Runnels

Old Lake Winters

Elm Creek

156 TX04313 San Antonio

Olmos Creek

Bexar

Olmos Reservoir

Palo Duro Creek

Hansford

Palo Duro Reservoir

Lamar

Pat Mayse Lake

32 13'06" 101'37'30" 1989

31 ${ }^{\circ} 57^{\prime} 00^{\prime \prime} \quad 96^{\circ} 42^{\prime} 00^{\prime \prime} \quad 1963$

$33^{\circ} 59^{\prime} 12^{\prime \prime} \quad 98^{\circ} 45^{\prime} 06^{\prime \prime} \quad 1964 \quad 33.4$

$32^{\circ} 56^{\prime} 48^{\prime \prime} \quad 96^{\circ} 58^{\prime} 12^{\prime \prime} \quad 1957 \quad 2.7$

$31^{\circ} 28^{\prime} 00^{\prime \prime} \quad 100^{\circ} 29^{\prime} 00^{\prime \prime} \quad 1952$

$32^{\circ} 02^{\prime} 24^{\prime \prime} \quad 100^{\circ} 16^{\prime} 00^{\prime \prime} \quad 1950 \quad 244$

31 $57^{\circ} 06^{\prime \prime} \quad 99^{\circ} 52^{\prime 24 " ~} 1945$

29॰28'24" 98²8'24" 1926 


\begin{tabular}{|c|c|c|c|c|c|c|c|c|c|c|c|}
\hline $\begin{array}{l}\text { Seq. } \\
\text { no. }\end{array}$ & $\begin{array}{c}\text { Maximum } \\
\text { capacity } \\
\text { (acre-ft) }\end{array}$ & $\begin{array}{l}\text { Normal } \\
\text { capacity } \\
\text { (acre-ft) }\end{array}$ & $\begin{array}{l}\text { Surface } \\
\text { area } \\
\text { (acres) }\end{array}$ & $\begin{array}{c}\text { Maximum } \\
\text { discharge } \\
\left(\mathrm{ft}^{3} / \mathrm{s}\right)\end{array}$ & $\begin{array}{l}\text { Type } \\
\text { of } \\
\text { spill- } \\
\text { way }\end{array}$ & $\begin{array}{l}\text { Pur- } \\
\text { pose } \\
\text { of } \\
\text { dam }\end{array}$ & Owner name & Owner address & $\begin{array}{l}\text { Ques- } \\
\text { tion- } \\
\text { aire } \\
\text { returned }\end{array}$ & $\begin{array}{l}\text { Updated } \\
\text { infor- } \\
\text { mation }\end{array}$ & $\begin{array}{l}\text { Outflow peak- } \\
\text { streamflow } \\
\text { frequency } \\
\left(\mathrm{ft}^{3} / \mathrm{s}\right)\end{array}$ \\
\hline 139 & $2,045,000$ & $1,788,000$ & 82,600 & 311,000 & $\mathrm{C}$ & $\mathrm{O}$ & Trinity River Authority & P.O. Box 360, Livingston, TX 77351 & $\mathrm{X}$ & $\begin{array}{l}\text { Max. dis.; dis. } \\
\text { freq.; } \\
\text { Project } \\
\text { Mgr., Bill } \\
\text { Holder }\end{array}$ & $\begin{array}{l}\mathrm{Q}_{2}=40,000 \\
\mathrm{Q}_{5}=70,000 \\
\mathrm{Q}_{10}=85,000 \\
\mathrm{Q}_{25}=107,000 \\
\mathrm{Q}_{50}=122,000 \\
\mathrm{Q}_{100}=137,000 \\
\mathrm{Q}_{250}=152,000 \\
\mathrm{Q}_{500}=167,000\end{array}$ \\
\hline 140 & 21,831 & 11,900 & 413 & $\begin{array}{l}84,062 \\
\text { (PMF) }\end{array}$ & $\mathrm{U}$ & $\mathrm{O}$ & City of Jacksboro & 111 E. Archer, Jacksboro, TX 76458 & $\mathrm{X}$ & $\begin{array}{l}\text { DA; SA; max. } \\
\text { dis; dis. } \\
\text { freq. }\end{array}$ & $\begin{array}{l}\mathrm{Q}_{5}=799 \\
\mathrm{Q}_{10}=884 \\
\mathrm{Q}_{25}=891 \\
\mathrm{Q}_{50}=4,729 \\
\mathrm{Q}_{100}=8,416 \\
\mathrm{Q}_{\mathrm{PMF}}=84,062\end{array}$ \\
\hline 141 & 16,491 & 3,774 & 209 & 22,336 & $\mathrm{U}$ & $\mathrm{FC}$ & Tarrant County WCID No. 1 & $\begin{array}{l}\text { P.O. Box 4508, Fort Worth, TX } \\
\quad 76102\end{array}$ & -- & -- & -- \\
\hline 142 & 182,300 & 77,619 & 5,440 & 45,300 & $\mathrm{C}$ & $\mathrm{O}$ & TU Electric & $\begin{array}{l}\text { Skyway Tower, } 400 \text { N. Olive St., } \\
\text { Dallas, TX } 75201\end{array}$ & -- & -- & -- \\
\hline 143 & 327,250 & 254,000 & 5,575 & 658,346 & $\mathrm{U}$ & $\mathrm{O}$ & $\begin{array}{l}\text { Bexar-Medina-Atascosa } \\
\text { Counties WCID } 1\end{array}$ & P.O. Box 170, Natalia, TX 78059 & -- & -- & -- \\
\hline 144 & 131,000 & 38,800 & 2,882 & 313,211 & $\mathrm{U}$ & $\mathrm{O}$ & North Central Texas MWD & P.O. Box 36, Munday, TX 76371 & $\mathrm{X}$ & $\begin{array}{l}\text { Owner, Dolan } \\
\text { Moore; DA; } \\
\max \\
\text { outflow }\end{array}$ & -- \\
\hline 145 & 50,241 & 32,000 & 1,603 & 19,608 & $\mathrm{U}$ & $\mathrm{O}$ & Colorado River MUD & P.O. Box 869, Big Spring, TX 79721 & -- & -- & -- \\
\hline 146 & 47,600 & 40,100 & -- & 38,000 & $\mathrm{U}$ & $\mathrm{O}$ & TU Electric & $\begin{array}{l}\text { Skyway Tower, } 400 \text { N. Olive St., } \\
\text { Dallas, TX } 75201\end{array}$ & -- & -- & -- \\
\hline 147 & 70,880 & 40,000 & 2,490 & 135,274 & $\mathrm{C}$ & $\mathrm{O}$ & TU Electric & $\begin{array}{l}\text { Skyway Tower, } 400 \text { N. Olive St., } \\
\text { Dallas, TX } 75201\end{array}$ & -- & -- & -- \\
\hline 148 & 92,000 & 44,650 & 3,890 & 26,700 & $\mathrm{U}$ & $\mathrm{O}$ & Panola County FWSD No. 1 & P.O. Box 331, Carthage, TX 75633 & $\mathrm{X}$ & $\begin{array}{l}\text { Chairman, J.R. } \\
\text { Jacks; SA; } \\
\text { max dis. }\end{array}$ & -- \\
\hline 149 & 207,265 & 54,560 & -- & -- & $\mathrm{U}$ & $\mathrm{FC}, \mathrm{O}$ & Colorado River MUD & P.O. Box 869, Big Spring, TX 79721 & -- & -- & -- \\
\hline 150 & 335,800 & 63,300 & -- & 224,000 & $\mathrm{C}$ & $\mathrm{FC}, \mathrm{O}$ & $\begin{array}{l}\text { U.S. Army Corps of } \\
\text { Engineers }\end{array}$ & $\begin{array}{l}\text { P.O. Box 17300, Fort Worth, TX } \\
76102\end{array}$ & -- & -- & -- \\
\hline 151 & 32,250 & 15,400 & -- & 34,271 & $\mathrm{U}$ & $\mathrm{O}$ & City of Iowa Park & P.O. Box 190, Iowa Park, TX 76367 & -- & -- & -- \\
\hline 152 & 24,000 & 16,000 & 806 & -- & $\mathrm{U}$ & $\mathrm{O}$ & TU Electric & $\begin{array}{l}\text { Skyway Tower, } 400 \text { N. Olive St., } \\
\text { Dallas, TX } 75201\end{array}$ & -- & -- & -- \\
\hline 153 & 696,300 & 119,200 & -- & 356,200 & $\mathrm{U}$ & $\mathrm{FC}, \mathrm{O}$ & $\begin{array}{l}\text { U.S. Army Corps of } \\
\text { Engineers }\end{array}$ & $\begin{array}{l}\text { P.O. Box 17300, Fort Worth, TX } \\
\quad 76102\end{array}$ & -- & -- & -- \\
\hline 154 & 79,336 & 39,360 & 2,375 & 112,300 & $\mathrm{U}$ & $\mathrm{O}$ & City of Sweetwater & $\begin{array}{l}\text { P.O. Box 450, Sweetwater, TX } \\
79556\end{array}$ & -- & -- & -- \\
\hline 155 & 10,032 & 2,447 & -- & -- & $\mathrm{U}$ & $\mathrm{O}$ & City of Winters & 310 S. Main, Winters, TX 79567 & $\mathrm{X}$ & -- & -- \\
\hline 156 & 14,240 & -- & 0 & -- & -- & FC & City of San Antonio & $\begin{array}{l}512 \text { Mission Rd., San Antonio, TX } \\
78210\end{array}$ & -- & -- & -- \\
\hline 157 & 278,500 & 60,897 & 2,413 & 237,500 & $\mathrm{U}$ & $\mathrm{O}$ & Palo Duro River Authority & P.O. Box 99, Spearman, TX 79081 & $\mathrm{X}$ & $\begin{array}{l}\text { DA; norm. } \\
\text { cap.; SA; } \\
\text { dis. freq. }\end{array}$ & $\begin{array}{l}\mathrm{Q}_{50}=2,911.4 \\
\mathrm{Q}_{100}=2,915.6\end{array}$ \\
\hline 158 & 457,800 & 118,110 & 5,940 & 5,720 & $\mathrm{U}$ & $\mathrm{FC}, \mathrm{O}$ & $\begin{array}{l}\text { U.S. Army Corps of } \\
\text { Engineers }\end{array}$ & P.O. Box 61, Tulsa, OK 74121 & $\mathrm{X}$ & All statistics & $\begin{array}{l}\mathrm{Q}_{2}=870 \\
\mathrm{Q}_{5}=915 \\
\mathrm{Q}_{10}=940 \\
\mathrm{Q}_{25}=950 \\
\mathrm{Q}_{50}=960\end{array}$ \\
\hline 159 & 13,336 & 6,853 & 176 & 2,537 & $\mathrm{U}$ & $\mathrm{O}$ & Lone Star Steel Co. & P.O. Box 1000, Lone Star, TX 75668 & $\mathrm{X}$ & $\begin{array}{l}\text { Address; ph.; } \\
\text { DA; norm. } \\
\text { cap.; SA; } \\
\text { max. cap. }\end{array}$ & -- \\
\hline 160 & 13,500 & 7,380 & -- & 5,620 & $\mathrm{U}$ & $\mathrm{O}$ & City of Center & P.O. Box 311, Center, TX 75935 & -- & -- & -- \\
\hline 161 & 556,220 & 556,220 & 17,700 & 500,600 & $\mathrm{C}$ & $\mathrm{O}$ & Brazos River Authority & P.O. Box 7555, Waco, TX 76714 & $\mathrm{X}$ & $\begin{array}{l}\text { DA; norm. } \\
\text { cap.; max. } \\
\text { cap.; max. } \\
\text { dis. }\end{array}$ & -- \\
\hline
\end{tabular}


Table 3. Texas Natural Resource Conservation Commission permitted dams with maximum capacities in excess

\begin{tabular}{|c|c|c|c|c|c|c|c|c|c|}
\hline $\begin{array}{l}\text { Seq. } \\
\text { no. }\end{array}$ & $\begin{array}{c}\text { TNRCC } \\
\text { no. } \\
\text { (pl. 2) }\end{array}$ & $\begin{array}{l}\text { River } \\
\text { basin }\end{array}$ & $\begin{array}{c}\text { Stream } \\
\text { name }\end{array}$ & County & $\begin{array}{c}\text { Reservoir } \\
\text { name }\end{array}$ & Latitude & Longitude & $\begin{array}{l}\text { Year } \\
\text { com- } \\
\text { pleted }\end{array}$ & $\begin{array}{l}\text { Drain- } \\
\text { age } \\
\text { area } \\
\left(\mathrm{mi}^{2}\right)\end{array}$ \\
\hline 162 & TX00010 & Brazos & Leon River & Comanche & Proctor Lake & $31^{\circ} 58^{\prime} 18^{\prime \prime}$ & $98^{\circ} 28^{\prime} 36^{\prime \prime}$ & 1963 & -- \\
\hline 163 & TX02250 & Rio Grande & Becerra Creek & Webb & Rancho Blanco Lake & $27^{\circ} 17^{\prime} 36^{\prime \prime}$ & $99^{\circ} 28^{\prime} 54^{\prime \prime}$ & 1943 & -- \\
\hline 164 & TX08008 & Trinity & Elm Fork & Denton & Ray Roberts Lake & $33^{\circ} 21^{\prime} 24^{\prime \prime}$ & $97^{\circ} 02^{\prime} 12^{\prime \prime}$ & 1986 & 692 \\
\hline 165 & TX02312 & Rio Grande & Pecos River & Reeves & Red Bluff Reservoir & $31^{\circ} 54^{\prime} 06^{\prime \prime}$ & $103^{\circ} 54^{\prime} 36^{\prime \prime}$ & 1936 & 20,760 \\
\hline 166 & TX02465 & Nueces-Rio Grande & Tranquitas Creek & Kleberg & Reservoir No. 6s & $27^{\circ} 33^{\prime} 30^{\prime \prime}$ & $97^{\circ} 56^{\prime} 00^{\prime \prime}$ & 1938 & -- \\
\hline 167 & TX03793 & Nueces-Rio Grande & Off-channel & Hidalgo & Retama Reservoir & $26^{\circ} 23^{\prime} 00^{\prime \prime}$ & $98^{\circ} 09^{\prime} 42^{\prime \prime}$ & 1935 & -- \\
\hline 168 & TX06316 & Trinity & Richland Creek & Freestone & Richland Creek Reservoir & $31^{\circ} 58^{\prime} 00^{\prime \prime}$ & $96^{\circ} 08^{\prime} 30^{\prime \prime}$ & 1987 & 1,957 \\
\hline 169 & TX03746 & Canadian & Rita Blanca Creek & Hartley & Rita Blanca Lake & $36^{\circ} 01^{\prime} 30^{\prime \prime}$ & $102^{\circ} 29^{\prime} 54^{\prime \prime}$ & 1940 & -- \\
\hline 170 & TX00011 & Neches & Angelina River & Jasper & Sam Rayburn Reservoir & $31^{\circ} 04^{\prime} 00^{\prime \prime}$ & $94^{\circ} 05^{\prime} 00^{\prime \prime}$ & 1965 & 3,449 \\
\hline 171 & TX00965 & Red & Beaver Creek & Wilbarger & Santa Rosa Lake & $33^{\circ} 56^{\prime} 24^{\prime \prime}$ & $99^{\circ} 15^{\prime} 36^{\prime \prime}$ & 1929 & 327.8 \\
\hline 172 & TX01384 & Brazos & Duck Creek & Dickens & SCS Lake 1 & $33^{\circ} 38^{\prime} 42^{\prime \prime}$ & $100^{\circ} 54^{\prime} 06^{\prime \prime}$ & 1968 & 20.49 \\
\hline 173 & TX04602 & Brazos & Hog Creek & Bosque & SCS Site 1 & $31^{\circ} 39^{\prime} 18^{\prime \prime}$ & $97^{\circ} 38^{\prime} 12^{\prime \prime}$ & 1977 & -- \\
\hline 174 & TX70111 & Brazos & Running Water Draw & Parmer & SCS Site 1 & 34--'--" & $103^{\circ---^{\prime}-{ }^{\prime}}$ & 1974 & 128.24 \\
\hline 175 & TX05945 & Guadalupe & Sink Creek & Hays & SCS Site 1 & $29^{\circ} 55^{\prime} 06^{\prime \prime}$ & $97^{\circ} 58^{\prime} 24^{\prime \prime}$ & 1983 & 33.6 \\
\hline 176 & TX02368 & Rio Grande & Johnson Draw & Crockett & SCS Site 1 & $30^{\circ} 52^{\prime} 24^{\prime \prime}$ & $101^{\circ} 12^{\prime} 42^{\prime \prime}$ & 1958 & 17 \\
\hline 177 & TX06717 & Rio Grande & Cornudas Draw & Hudspeth & SCS Site 1 & $31^{\circ} 58^{\prime} 44^{\prime \prime}$ & $105^{\circ} 16^{\prime} 32^{\prime \prime}$ & 1982 & 97.1 \\
\hline 178 & TX04481 & San Antonio & Cibolo Creek & Kendall & SCS Site 1 & $29^{\circ} 49^{\prime} 18^{\prime \prime}$ & $98^{\circ} 46^{\prime} 00^{\prime \prime}$ & 1978 & 19.8 \\
\hline 179 & TX04716 & San Antonio & Salado Creek & Bexar & SCS Site 1 & $29^{\circ} 39^{\prime} 48^{\prime \prime}$ & $98^{\circ} 36^{\prime} 00^{\prime \prime}$ & 1975 & 11.3 \\
\hline 180 & TX01677 & Colorado & South Brady Creek & McCulloch & SCS Site 17 & $31^{\circ} 08^{\prime} 48^{\prime \prime}$ & $99^{\circ} 35^{\prime} 48^{\prime \prime}$ & 1962 & 28.8 \\
\hline 181 & TX04747 & Trinity & Brushy Elm Creek & Cooke & SCS Site 19 & $33^{\circ} 39^{\prime} 24^{\prime \prime}$ & $97^{\circ} 23^{\prime} 54^{\prime \prime}$ & -- & -- \\
\hline 182 & TX00207 & Trinity & Turkey Creek & Henderson & SCS Site 2 & $32^{\circ} 05^{\prime} 54^{\prime \prime}$ & $95^{\circ} 59^{\prime} 48^{\prime \prime}$ & 1955 & 19.8 \\
\hline 183 & TX00846 & Brazos & Gilmore Creek & Erath & SCS Site 27 & $31^{\circ} 59^{\prime} 00^{\prime \prime}$ & $98^{\circ} 06^{\prime} 42^{\prime \prime}$ & 1973 & 20.7 \\
\hline 184 & TX01626 & Colorado & Fitzgerald Creek & Concho & SCS Site 28 & $31^{\circ} 08^{\prime} 54^{\prime \prime}$ & $99^{\circ} 52^{\prime} 48^{\prime \prime}$ & 1957 & 21.88 \\
\hline 185 & TX02367 & Rio Grande & Garrett Draw & Crockett & SCS Site 3 & $30^{\circ} 50^{\prime} 00^{\prime \prime}$ & $101^{\circ} 16^{\prime} 06^{\prime \prime}$ & 1958 & 16.7 \\
\hline 186 & TХ02299 & Rio Grande & Dry Devils River & Sutton & SCS Site 3 & $30^{\circ} 41^{\prime} 42^{\prime \prime}$ & $100^{\circ} 41^{\prime} 00^{\prime \prime}$ & 1961 & 23.93 \\
\hline 187 & TX04827 & Brazos & Running Water Draw & Hale & SCS Site 3 & $34^{\circ} 15^{\prime} 00^{\prime \prime}$ & $101^{\circ} 53^{\prime} 36^{\prime \prime}$ & 1982 & 390.2 \\
\hline 188 & TX04715 & Brazos & Running Water Draw & Parmer & SCS Site 3 & $34^{\circ} 24^{\prime} 12^{\prime \prime}$ & $102^{\circ} 31^{\prime} 42^{\prime \prime}$ & 1979 & 123.9 \\
\hline 189 & TX01625 & Colorado & Brady Creek & Concho & SCS Site 31 & $31^{\circ} 10^{\prime} 06^{\prime \prime}$ & $99^{\circ} 58^{\prime} 30^{\prime \prime}$ & 1958 & 22.5 \\
\hline 190 & TX04530 & Nueces-Rio Grande & Chiltipin Creek & Jim Wells & SCS Site 4 & $27^{\circ} 53^{\prime} 36^{\prime \prime}$ & $98^{\circ} 10^{\prime} 00^{\prime \prime}$ & 1974 & 37.6 \\
\hline 191 & TX01468 & San Antonio & Panther Springs Creek & Bexar & SCS Site 4 & $29^{\circ} 37^{\prime} 24^{\prime \prime}$ & $98^{\circ} 31^{\prime} 12^{\prime \prime}$ & 1972 & 5.5 \\
\hline 192 & TX03612 & Trinity & Turkey Creek & Johnson & SCS Site 42 & $32^{\circ} 22^{\prime} 18^{\prime \prime}$ & $97^{\circ} 13^{\prime} 54^{\prime \prime}$ & 1966 & 15.3 \\
\hline 193 & TX03350 & Trinity & Big Cottonwood Creek & Kaufman & SCS Site 60 & $32^{\circ} 35^{\prime} 06^{\prime \prime}$ & $96^{\circ} 15^{\prime} 36^{\prime \prime}$ & 1962 & 8.32 \\
\hline 194 & TX02940 & Colorado & North Prong Pecan Bayou & Callahan & SCS Site 7 & $32^{\circ} 18^{\prime} 48^{\prime \prime}$ & $99^{\circ} 28^{\prime} 12^{\prime \prime}$ & 1970 & 37.9 \\
\hline 195 & TX03341 & Trinity & Muddy Cedar Creek & Kaufman & SCS Site $87 \mathrm{~A}$ & $32^{\circ} 43^{\prime} 42^{\prime \prime}$ & $96^{\circ} 10^{\prime} 24^{\prime \prime}$ & 1955 & 14.33 \\
\hline
\end{tabular}




\section{of 10,000 acre-feet-Continued}

\begin{tabular}{|c|c|c|c|c|c|c|c|c|c|c|c|}
\hline $\begin{array}{l}\text { Seq. } \\
\text { no. }\end{array}$ & $\begin{array}{c}\text { Maximum } \\
\text { capacity } \\
\text { (acre-ft) }\end{array}$ & $\begin{array}{l}\text { Normal } \\
\text { capacity } \\
\text { (acre-ft) }\end{array}$ & $\begin{array}{l}\text { Surface } \\
\text { area } \\
\text { (acres) }\end{array}$ & $\begin{array}{c}\text { Maximum } \\
\text { discharge } \\
\left(\mathrm{ft}^{3} / \mathrm{s}\right)\end{array}$ & $\begin{array}{l}\text { Type } \\
\text { of } \\
\text { spill- } \\
\text { way }\end{array}$ & $\begin{array}{l}\text { Pur- } \\
\text { pose } \\
\text { of } \\
\text { dam }\end{array}$ & Owner name & Owner address & $\begin{array}{l}\text { Ques- } \\
\text { tion- } \\
\text { aire } \\
\text { returned }\end{array}$ & $\begin{array}{l}\text { Updated } \\
\text { infor- } \\
\text { mation }\end{array}$ & $\begin{array}{l}\text { Outflow peak- } \\
\text { streamflow } \\
\text { frequency } \\
\left(\mathrm{ft}^{3} / \mathrm{s}\right)\end{array}$ \\
\hline 162 & 433,000 & 59,400 & -- & 431,800 & $\mathrm{C}$ & $\mathrm{FC}, \mathrm{O}$ & $\begin{array}{l}\text { U.S. Army Corps of } \\
\text { Engineers }\end{array}$ & $\begin{array}{l}\text { P.O. Box 17300, Fort Worth, TX } \\
\quad 76102\end{array}$ & -- & -- & -- \\
\hline 163 & 21,500 & 1,730 & -- & -- & $\mathrm{U}$ & $\mathrm{O}$ & Rancho Blanco & $\begin{array}{l}\text { P.O. Box 21130, San Antonio, TX } \\
\quad 78221\end{array}$ & -- & -- & -- \\
\hline 164 & $1,931,900$ & 799,600 & 29,350 & 21,600 & $\mathrm{U}$ & $\mathrm{FC}, \mathrm{O}$ & $\begin{array}{l}\text { U.S. Army Corps of } \\
\text { Engineers }\end{array}$ & $\begin{array}{l}\text { P.O. Box 17300, Fort Worth, TX } \\
\quad 76102\end{array}$ & -- & -- & -- \\
\hline 165 & 518,950 & 308,180 & 7,507 & 389,749 & $\mathrm{U}$ & $\mathrm{O}$ & $\begin{array}{l}\text { Red Bluff Water Power } \\
\text { District }\end{array}$ & 111 W. Second St., Pecos, TX 79772 & -- & -- & -- \\
\hline 166 & 18,316 & 1,147 & -- & 22,681 & $\mathrm{U}$ & $\mathrm{O}$ & King Ranch, Inc. & $\begin{array}{l}\text { P.O. Box 1090, Kingsville, TX } \\
78364\end{array}$ & -- & -- & -- \\
\hline 167 & 13,500 & 8,864 & -- & -- & -- & $\mathrm{O}$ & $\begin{array}{l}\text { Santa Cruz Irrigation } \\
\text { District No. } 15\end{array}$ & P.O. Box 599, Edinburg, TX 78540 & -- & -- & -- \\
\hline 168 & $1,743,000$ & $1,135,000$ & 44,752 & 600,000 & $\mathrm{C}$ & $\mathrm{O}$ & Tarrant County WCID No. 1 & $\begin{array}{l}\text { P.O. Box } 4508 \text {, Fort Worth, TX } \\
\quad 76102\end{array}$ & -- & -- & -- \\
\hline 169 & 13,600 & 11,507 & -- & -- & $\mathrm{U}$ & $\mathrm{O}$ & Hartley-Dallam Counties & $\begin{array}{l}\text { Hartley County Courthouse, } \\
\text { Channing, TX } 79018\end{array}$ & -- & -- & -- \\
\hline 170 & $6,520,245$ & $2,898,200$ & -- & 125,300 & $\mathrm{U}$ & $\mathrm{FC}, \mathrm{O}$ & $\begin{array}{l}\text { U.S. Army Corps of } \\
\text { Engineers }\end{array}$ & $\begin{array}{l}\text { P.O. Box 17300, Fort Worth, TX } \\
76102\end{array}$ & -- & -- & -- \\
\hline 171 & 28,792 & 9,561 & 1,582 & 165,541 & $\mathrm{U}$ & $\mathrm{O}$ & W.T. Waggoner Estate & P.O. Box 2130, Vernon, TX 76384 & $\mathrm{X}$ & SA & -- \\
\hline 172 & 10,750 & 634 & 79.4 & 23,110 & $\mathrm{U}$ & $\mathrm{FC}$ & $\begin{array}{l}\text { Dickens County WCID } \\
\text { No. } 1\end{array}$ & 312 Willara Ave., Spur, TX 79370 & -- & -- & -- \\
\hline 173 & 12,119 & 740 & 74 & 19,893 & $\mathrm{U}$ & $\mathrm{FC}, \mathrm{O}$ & Bosque SWCD & Rt. 2, Box 82, Bluff Dale, TX 76433 & -- & -- & -- \\
\hline 174 & 25,150 & 2,170 & 235 & 40,173 & $\mathrm{U}$ & $\mathrm{FC}$ & Central Curry SWCD & -- & -- & -- & -- \\
\hline 175 & 18,399 & 107 & 25 & 76,527 & $\mathrm{U}$ & $\mathrm{FC}$ & $\begin{array}{l}\text { Comal-Hays-Guadalupe } \\
\text { SWCD }\end{array}$ & P.O. Box 992, Seguin, TX 78155 & -- & -- & -- \\
\hline 176 & 11,550 & 166 & 51 & 20,059 & $\mathrm{U}$ & $\mathrm{FC}$ & Crockett SWCD & P.O. Box 39, Ozona, TX 76943 & $\mathrm{X}$ & -- & -- \\
\hline 177 & 11,757 & 575 & 143 & 16,119 & $\mathrm{U}$ & $\mathrm{FC}$ & El Paso-Hudspeth SWCD & $\begin{array}{l}11930 \text { Vista del Sol, Ste. B, El Paso, } \\
\text { TX } 79936\end{array}$ & -- & -- & -- \\
\hline 178 & 15,668 & 4,043 & 189 & 43,756 & $\mathrm{U}$ & $\mathrm{FC}, \mathrm{O}$ & Kendall SWCD & $\begin{array}{l}\text { Rt. 2, Box 2486C, Boerne, TX } \\
\quad 78006\end{array}$ & -- & -- & -- \\
\hline 179 & $2,800,108$ & 199 & 25 & $2,800,108$ & $\mathrm{U}$ & $\mathrm{FC}$ & $\begin{array}{l}\text { San Antonio River } \\
\text { Authority }\end{array}$ & $\begin{array}{l}\text { P.O. Box 830027, San Antonio, TX } \\
\quad 78282\end{array}$ & -- & -- & -- \\
\hline 180 & 13,511 & 277 & 76 & 13,850 & $\mathrm{U}$ & $\mathrm{FC}$ & McCulloch SWCD & 200A E. 11th St., Brady, TX 76825 & -- & -- & -- \\
\hline 181 & 11,700 & 4,700 & -- & 12,353 & $\mathrm{U}$ & $\mathrm{FC}, \mathrm{O}$ & Upper Elm-Red SWCD & $\begin{array}{l}\text { Rt. 2, Box 34-8, Gainsville, TX } \\
\quad 75090\end{array}$ & $\mathrm{X}$ & Address; ph. & -- \\
\hline 182 & 10,500 & 433 & 121 & -- & $\mathrm{U}$ & $\mathrm{FC}$ & Trinity-Neches SWCD & 209 Dallas Hwy., Athens, TX 75751 & -- & -- & -- \\
\hline 183 & 10,169 & 1,227 & 125 & 53,649 & $\mathrm{U}$ & $\mathrm{FC}, \mathrm{O}$ & $\begin{array}{l}\text { Erath County } \\
\text { Commissioners Court }\end{array}$ & $\begin{array}{l}\text { Erath County Courthouse, } \\
\text { Stephenville, TX } 76401\end{array}$ & -- & -- & -- \\
\hline 184 & 13,042 & 200 & 67 & -- & $\mathrm{U}$ & $\mathrm{FC}$ & Concho SWCD & P.O. Box 392, Eden, TX 76837 & -- & -- & -- \\
\hline 185 & 10,723 & 174 & 50 & 19,277 & $\mathrm{U}$ & $\mathrm{FC}$ & Crockett SWCD & P.O. Box 39, Ozona, TX 76943 & $\mathrm{X}$ & -- & -- \\
\hline 186 & 10,643 & 200 & 50 & 84,775 & $\mathrm{U}$ & $\mathrm{FC}$ & Edwards Plateau SWCD & $\begin{array}{l}\text { 301 S.E. Crockett, Sonora, TX } \\
76950\end{array}$ & $\mathrm{X}$ & No info. & -- \\
\hline 187 & 14,312 & 8,213 & 54 & 8,875 & $\mathrm{U}$ & $\mathrm{FC}$ & Hale County SWCD & P.O. Box 312, Plainview, TX 79072 & -- & -- & -- \\
\hline 188 & 18,499 & 4,427 & 233 & 8,920 & $\mathrm{U}$ & $\mathrm{FC}, \mathrm{O}$ & Parmer County SWCD & $\begin{array}{l}1306 \text { W. Ninth St., Friona, TX } \\
79035\end{array}$ & $\mathrm{X}$ & -- & -- \\
\hline 189 & 11,155 & 189 & -- & 8,250 & $\mathrm{U}$ & $\mathrm{FC}$ & Concho SWCD & P.O. Box 392, Eden, TX 76837 & -- & -- & -- \\
\hline 190 & 17,023 & 200 & 65 & 41,186 & $\mathrm{U}$ & $\mathrm{FC}$ & $\begin{array}{l}\text { Nueces-Jim Wells-Kleberg- } \\
\text { Kenedy SWCD }\end{array}$ & $\begin{array}{l}548 \text { SH 77, Ste. B, Robstown, TX } \\
78380\end{array}$ & $\mathrm{X}$ & -- & -- \\
\hline 191 & 30,798 & 85 & 16 & 230,208 & $\mathrm{U}$ & FC & $\begin{array}{l}\text { San Antonio River } \\
\text { Authority }\end{array}$ & $\begin{array}{l}\text { P.O. Box 830027, San Antonio, TX } \\
\quad 78282\end{array}$ & -- & -- & -- \\
\hline 192 & 14,107 & 4,757 & 503 & 48,244 & $\mathrm{U}$ & $\mathrm{FC}, \mathrm{O}$ & Johnson County SWCD & P.O. Box 293, Cleburne, TX 76033 & $\mathrm{X}$ & -- & -- \\
\hline 193 & 16,800 & 1,834 & 330.3 & -- & $\mathrm{U}$ & $\mathrm{FC}, \mathrm{O}$ & $\begin{array}{l}\text { Kaufman-Van Zandt County } \\
\text { SWCD }\end{array}$ & $\begin{array}{l}105 \text { E. Sixth St., Kaufman, TX } \\
75142\end{array}$ & -- & -- & -- \\
\hline 194 & 16,550 & 5,748 & 449 & 21,870 & $\mathrm{U}$ & $\mathrm{O}$ & Callahan Divide SWCD & $\begin{array}{l}\text { Rt. 1, Box 160-B, Cross Plains, TX } \\
\quad 76443\end{array}$ & $\mathrm{X}$ & -- & -- \\
\hline 195 & 20,147 & 8,712 & 840 & 22,468 & $\mathrm{U}$ & $\mathrm{FC}, \mathrm{O}$ & $\begin{array}{l}\text { Kaufman-Van Zandt County } \\
\text { SWCD }\end{array}$ & $\begin{array}{l}105 \text { E. Sixth St., Kaufman, TX } \\
75142\end{array}$ & -- & -- & -- \\
\hline
\end{tabular}


Table 3. Texas Natural Resource Conservation Commission permitted dams with maximum capacities in excess

\begin{tabular}{|c|c|c|c|c|c|c|c|c|c|}
\hline $\begin{array}{l}\text { Seq. } \\
\text { no. }\end{array}$ & $\begin{array}{c}\text { TNRCC } \\
\text { no. } \\
\text { (pl. 2) }\end{array}$ & $\begin{array}{l}\text { River } \\
\text { basin }\end{array}$ & $\begin{array}{l}\text { Stream } \\
\text { name }\end{array}$ & County & $\begin{array}{l}\text { Reservoir } \\
\text { name }\end{array}$ & Latitude & Longitude & $\begin{array}{c}\text { Year } \\
\text { com- } \\
\text { pleted }\end{array}$ & $\begin{array}{l}\text { Drain- } \\
\text { age } \\
\text { area } \\
\left(\mathrm{mi}^{2}\right)\end{array}$ \\
\hline 196 & TX04788 & Guadalupe & Dry Comal Creek & Comal & SCS Site 2 & $29^{\circ} 40^{\prime} 30^{\prime \prime}$ & $98^{\circ} 15^{\prime} 06^{\prime \prime}$ & 1981 & 30.1 \\
\hline 197 & TX03415 & San Jacinto & Carpenters Bayou & Harris & Sheldon Reservoir & $29^{\circ} 51^{\prime} 12^{\prime \prime}$ & $95^{\circ} 10^{\prime} 00^{\prime \prime}$ & 1944 & 6.6 \\
\hline 198 & TX01564 & Brazos & Dry Creek & Fort Bend & Smithers Lake & $29^{\circ} 28^{\prime} 48^{\prime \prime}$ & $95^{\circ} 37^{\prime} 48^{\prime \prime}$ & 1957 & -- \\
\hline 199 & TX04627 & Brazos & Squaw Creek & Somervell & Squaw Creek Reservoir & $32^{\circ} 17^{\prime} 18^{\prime \prime}$ & $97^{\circ} 45^{\prime} 36^{\prime \prime}$ & 1977 & 64 \\
\hline 200 & TX00014 & Brazos & Lampasas River & Bell & Stillhouse Hollow Reservoir & $31^{\circ} 02^{\prime} 00^{\prime \prime}$ & $97^{\circ} 32^{\prime} 00^{\prime \prime}$ & 1968 & -- \\
\hline 201 & TX06482 & Colorado & Sulphur Springs Draw & Martin & Sulphur Springs Draw Storage Reservoir & $32^{\circ} 19^{\prime} 18^{\prime \prime}$ & $101^{\circ} 44^{\prime} 56^{\prime \prime}$ & 1993 & 258 \\
\hline 202 & LA00030 & Sabine & Sabine River & Newton & Toledo Bend Reservoir & $31^{\circ} 10^{\prime} 42^{\prime \prime}$ & $93^{\circ} 34^{\prime} 00^{\prime \prime}$ & 1968 & -- \\
\hline 203 & TX04110 & Brazos & Tradinghouse Creek & McLennan & Tradinghouse Creek Reservoir & $31^{\circ} 33^{\prime} 12^{\prime \prime}$ & $96^{\circ} 58^{\prime} 48^{\prime \prime}$ & 1968 & -- \\
\hline 204 & TX05996 & Red & Bluff Creek & Knox & Truscott Brine Lake & $33^{\circ} 47^{\prime} 54^{\prime \prime}$ & $99^{\circ} 50^{\prime} 12^{\prime \prime}$ & 1982 & 26.2 \\
\hline 205 & TX04316 & Red & Tule Creek & Briscoe & Tule Creek Lake & $34^{\circ} 32^{\prime} 36^{\prime \prime}$ & $101^{\circ} 26^{\prime} 12^{\prime \prime}$ & 1974 & 188 \\
\hline 206 & TX00022 & Colorado & $\begin{array}{l}\text { Middle and South Concho } \\
\text { Rivers }\end{array}$ & Tom Green & Twin Buttes Reservoir & $31^{\circ} 22^{\prime} 36^{\prime \prime}$ & $100^{\circ} 32^{\prime} 00^{\prime \prime}$ & 1963 & 2,980 \\
\hline 207 & TX04453 & Brazos & Duck Creek & Robertson & Twin Oak Reservoir & $31^{\circ} 12^{\prime} 00^{\prime \prime}$ & $96^{\circ} 27^{\prime} 48^{\prime \prime}$ & 1982 & -- \\
\hline 208 & TX06588 & Brazos & Big Elm Creek & Bell & $\begin{array}{l}\text { Unnamed trib.-Elm Creek Watershed SCS } \\
\text { Site } 1 \text { Dam }\end{array}$ & $31^{\circ} 14^{\prime} 30^{\prime \prime}$ & $97^{\circ} 18^{\prime} 48^{\prime \prime}$ & 1984 & 21.12 \\
\hline 209 & TX06458 & Nueces & Leona River & Uvalde & $\begin{array}{l}\text { Unnamed trib.-Leona River Watershed } \\
\text { SCS Site } 3\end{array}$ & $29^{\circ} 17^{\prime} 42^{\prime \prime}$ & $99^{\circ} 45^{\prime} 54^{\prime \prime}$ & 1983 & 37 \\
\hline 210 & TX06780 & Rio Grande & Sanderson Canyon & Pecos & $\begin{array}{l}\text { Unnamed trib.-Sanderson Canyon } \\
\text { Watershed SCS Site } 2\end{array}$ & $30^{\circ} 09^{\prime} 36^{\prime \prime}$ & $102^{\circ} 40^{\prime} 48^{\prime \prime}$ & 1986 & 53.23 \\
\hline 211 & TX01802 & Nueces & Nueces River & Zavala & Upper Nueces Lake & $28^{\circ} 46^{\prime} 42^{\prime \prime}$ & $99^{\circ} 49^{\prime} 42^{\prime \prime}$ & 1947 & 350 \\
\hline 212 & TX00418 & Red & Brushy Creek & Fannin & Valley Lake & $33^{\circ} 38^{\prime} 42^{\prime \prime}$ & $96^{\circ} 21^{\prime} 30^{\prime \prime}$ & 1961 & -- \\
\hline 213 & TX04357 & Cypress & Swauno Creek & Titus & Welsh Reservoir & $33^{\circ} 02^{\prime} 36^{\prime \prime}$ & $94^{\circ} 50^{\prime} 00^{\prime \prime}$ & 1975 & -- \\
\hline 214 & TX02143 & Brazos & White River & Crosby & White River Lake & $33^{\circ} 27^{\prime} 24^{\prime \prime}$ & $101^{\circ} 05^{\prime} 00^{\prime \prime}$ & 1963 & -- \\
\hline 215 & TX00840 & Trinity & White Rock Creek & Dallas & White Rock Lake & $32^{\circ} 48^{\prime} 54^{\prime \prime}$ & $96^{\circ} 43^{\prime} 30^{\prime \prime}$ & 1911 & -- \\
\hline 216 & TX05776 & Colorado & Elm Creek & Runnels & Winters Elm Creek Reservoir & $31^{\circ} 56^{\prime} 18^{\prime \prime}$ & $99^{\circ} 52^{\prime} 06^{\prime \prime}$ & 1983 & 65.5 \\
\hline 217 & TX00021 & Sulphur & Sulphur River & Bowie & Wright Patman Lake & $33^{\circ} 18^{\prime} 18^{\prime \prime}$ & $94^{\circ} 09^{\prime} 36^{\prime \prime}$ & 1957 & 5.38 \\
\hline
\end{tabular}




\section{of 10,000 acre-feet-Continued}

\begin{tabular}{|c|c|c|c|c|c|c|c|c|c|c|c|}
\hline $\begin{array}{l}\text { Seq. } \\
\text { no. }\end{array}$ & $\begin{array}{l}\text { Maximum } \\
\text { capacity } \\
\text { (acre-ft) }\end{array}$ & $\begin{array}{l}\text { Normal } \\
\text { capacity } \\
\text { (acre-ft) }\end{array}$ & $\begin{array}{c}\text { Surface } \\
\text { area } \\
\text { (acres) }\end{array}$ & $\begin{array}{c}\text { Maximum } \\
\text { discharge } \\
\left(\mathrm{ft}^{3} / \mathrm{s}\right)\end{array}$ & $\begin{array}{l}\text { Type } \\
\text { of } \\
\text { spill- } \\
\text { way }\end{array}$ & $\begin{array}{l}\text { Pur- } \\
\text { pose } \\
\text { of } \\
\text { dam }\end{array}$ & Owner name & Owner address & $\begin{array}{l}\text { Ques- } \\
\text { tion- } \\
\text { aire } \\
\text { returned }\end{array}$ & $\begin{array}{l}\text { Updated } \\
\text { infor- } \\
\text { mation }\end{array}$ & $\begin{array}{l}\text { Outflow peak- } \\
\text { streamflow } \\
\text { frequency } \\
\left(\mathrm{ft}^{3} / \mathrm{s}\right)\end{array}$ \\
\hline 196 & 19,024 & 177 & 32 & 83,171 & $\mathrm{U}$ & $\mathrm{FC}, \mathrm{O}$ & $\begin{array}{l}\text { Comal County } \\
\text { Commissioners Court }\end{array}$ & $\begin{array}{l}150 \text { N. Seguin, Ste. 301, } \\
\text { New Braunfels, TX } 78130\end{array}$ & -- & -- & -- \\
\hline 197 & 21,138 & 4,224 & 1,244 & 4,033 & $\mathrm{U}$ & $\mathrm{O}$ & $\begin{array}{l}\text { Texas Parks and Wildlife } \\
\text { Department }\end{array}$ & $\begin{array}{l}4200 \text { Smith School Rd., Austin, TX } \\
78744\end{array}$ & $\mathrm{X}$ & -- & -- \\
\hline 198 & 18,750 & 18,750 & -- & -- & $\mathrm{C}$ & $\mathrm{FC}, \mathrm{O}$ & $\begin{array}{l}\text { Houston Lighting and } \\
\text { Power Co. }\end{array}$ & P.O. Box 1700, Houston, TX 77001 & -- & -- & -- \\
\hline 199 & 199,427 & 151,047 & 3,228 & 87,128 & $\mathrm{U}$ & $\mathrm{O}$ & TU Electric & $\begin{array}{l}\text { Skyway Tower, } 400 \text { N. Olive St., } \\
\text { Dallas, TX } 75201\end{array}$ & -- & -- & -- \\
\hline 200 & $1,013,300$ & 235,700 & -- & 673,500 & $\mathrm{U}$ & $\mathrm{FC}, \mathrm{O}$ & $\begin{array}{l}\text { U.S. Army Corps of } \\
\text { Engineers }\end{array}$ & $\begin{array}{l}\text { P.O. Box 17300, Fort Worth, TX } \\
\quad 76102\end{array}$ & -- & -- & -- \\
\hline 201 & 20,692 & 7,997 & 970 & 210,870 & $\mathrm{U}$ & $\mathrm{O}$ & Colorado River MUD & P.O. Box 869, Big Spring, TX 79721 & -- & -- & -- \\
\hline 202 & $5,100,000$ & $4,477,000$ & -- & 286,000 & $\mathrm{C}$ & $\mathrm{O}$ & $\begin{array}{l}\text { Sabine River Authorities of } \\
\text { Texas and Louisiana }\end{array}$ & $\begin{array}{l}\text { Toledo Bend Project Joint } \\
\text { Operation, Rt. 1, Box 270, } \\
\text { Burkeville, TX } 75932\end{array}$ & -- & -- & -- \\
\hline 203 & 57,032 & 37,814 & -- & 64,629 & $\mathrm{U}$ & $\mathrm{O}$ & TU Electric & $\begin{array}{l}\text { Skyway Tower, } 400 \text { N. Olive St., } \\
\text { Dallas, TX } 75201\end{array}$ & -- & -- & -- \\
\hline 204 & 135,000 & 111,147 & 3,146 & 35,400 & $\mathrm{U}$ & $\mathrm{O}$ & $\begin{array}{l}\text { U.S. Army Corps of } \\
\text { Engineers }\end{array}$ & P.O. Box 61, Tulsa, OK 74121 & $\mathrm{X}$ & $\begin{array}{l}\text { Yr. com- } \\
\text { pleted; } \\
\text { norm. cap.; } \\
\text { max. cap.; } \\
\text { max. dis.; } \\
\text { dis. freq. }\end{array}$ & -- \\
\hline 205 & 69,249 & 46,077 & 896 & 77,900 & $\mathrm{U}$ & $\mathrm{O}$ & Mackenzie MWA & Rt. 1, Box 14, Silverton, TX 79257 & $\mathrm{X}$ & $\begin{array}{l}\text { Owner, Roy F. } \\
\text { Garris; DA }\end{array}$ & -- \\
\hline 206 & $1,088,000$ & 186,200 & 9,080 & 47,300 & $\mathrm{U}$ & $\mathrm{FC}, \mathrm{O}$ & Bureau of Reclamation & P.O. Box 1946, Austin, TX 78767 & -- & -- & -- \\
\hline 207 & 70,885 & 30,319 & -- & 48,800 & $\mathrm{U}$ & $\mathrm{O}$ & TU Electric & $\begin{array}{l}\text { Skyway Tower, } 400 \text { N. Olive St., } \\
\text { Dallas, TX } 75201\end{array}$ & -- & -- & -- \\
\hline 208 & 18,300 & 1,867 & 32 & 32,800 & $\mathrm{U}$ & $\mathrm{FC}$ & Central Texas SWCD & 202 S. Main, Temple, TX 76501 & -- & -- & -- \\
\hline 209 & 21,306 & 185 & -- & -- & $\mathrm{U}$ & $\mathrm{FC}$ & Nueces-Frio-Sabinal SWCD & P.O. Box 146, Sabinal, TX 78802 & -- & -- & -- \\
\hline 210 & 11,789 & 1,874 & 51 & 35,102 & $\mathrm{U}$ & FC & $\begin{array}{l}\text { Rio Grande-Pecos River } \\
\text { SWCD }\end{array}$ & P.O. Box 140, Sanderson, TX 79848 & -- & -- & -- \\
\hline 211 & - & 4,010 & 25 & 100 & $\mathrm{U}$ & $\mathrm{O}$ & $\begin{array}{l}\text { Zavala-Dimmit Counties } \\
\text { WID No. } 1\end{array}$ & $\begin{array}{l}\text { P.O. Box 729, Crystal City, TX } \\
\quad 78839\end{array}$ & $\mathrm{X}$ & $\begin{array}{l}\text { DA; norm. } \\
\text { cap.; max. } \\
\text { cap.; SA; } \\
\text { max. dis. }\end{array}$ & -- \\
\hline 212 & 20,000 & 16,400 & -- & 4,600 & $\mathrm{U}$ & $\mathrm{O}$ & TU Electric & $\begin{array}{l}\text { Skyway Tower, } 400 \text { N. Olive St., } \\
\text { Dallas, TX } 75201\end{array}$ & -- & -- & -- \\
\hline 213 & 50,300 & 23,587 & -- & 21,840 & $\mathrm{U}$ & $\mathrm{O}$ & $\begin{array}{l}\text { Southwestern Electric } \\
\text { Power Co. }\end{array}$ & $\begin{array}{l}\text { P.O. Box 21106, Shreveport, LA } \\
\quad 71156\end{array}$ & -- & -- & -- \\
\hline 214 & 80,000 & 44,897 & -- & 91,120 & $\mathrm{U}$ & $\mathrm{O}$ & White River MUD & Star Rt. 2, Spur, TX 79370 & -- & -- & -- \\
\hline 215 & 39,400 & 10,743 & -- & 190,808 & $\mathrm{U}$ & $\mathrm{O}$ & City of Dallas & City Hall, Dallas, TX 75201 & -- & -- & -- \\
\hline 216 & 33,500 & 8,374 & 643 & 102,236 & $\mathrm{U}$ & $\mathrm{O}$ & City of Winters & 310 S. Main, Winters, TX 79567 & $\mathrm{X}$ & -- & -- \\
\hline 217 & $6,505,020$ & 145,300 & 20,300 & 63,200 & $\mathrm{U}$ & $\mathrm{FC}, \mathrm{O}$ & $\begin{array}{l}\text { U.S. Army Corps of } \\
\text { Engineers }\end{array}$ & $\begin{array}{l}\text { P.O. Box 17300, Fort Worth, TX } \\
\quad 76102\end{array}$ & -- & -- & -- \\
\hline
\end{tabular}


Table 4. Regression equations for estimation of L-moments and L-moment ratios of annual peak streamflow for three regions in Texas

[CV, coefficient of variation; A, contributing drainage area in square miles $\left(\mathrm{mi}^{2}\right)$; $\mathrm{SH}$, basin shape factor-ratio of length of longest mapped channel (stream

$\underset{\Rightarrow}{*}$ length) squared to contributing drainage area (dimensionless); SL, stream slope in feet per mile (ft/mi) —ratio of change in elevation of (1) longest mapped channel from site (or station) to headwaters to (2) length of longest mapped channel; --, not applicable]

\begin{tabular}{|c|c|c|c|c|c|c|c|c|}
\hline $\begin{array}{c}\text { Region and } \\
\text { L-moment } \\
\text { statistic }\end{array}$ & $\begin{array}{l}\text { Weighted least-squares } \\
\text { regression equation for } \\
\text { corresponding L-moment } \\
\text { statistic }\end{array}$ & $\begin{array}{l}\text { Regional mean (and } \\
\text { standard deviation) } \\
\text { of corresponding } \\
\text { L-moment statistic } \\
\text { (log } \log _{10} \text { or dimensionless) }\end{array}$ & $\begin{array}{l}\text { Range of indicated } \\
\text { independent } \\
\text { variables in } \\
\text { corresponding } \\
\text { region } \\
\text { (units as noted) }\end{array}$ & $\begin{array}{c}\text { Adjusted } \\
\text { R-squared }^{1}\end{array}$ & $\begin{array}{l}\text { Weighted } \\
\text { standard } \\
\text { error of } \\
\text { estimate } \\
\text { (percent) }\end{array}$ & $\begin{array}{c}\text { Weighted } \\
\text { standard error } \\
\text { of estimate } \\
\text { ( } \log _{10} \text { or } \\
\text { dimensionless) }\end{array}$ & $\begin{array}{l}\text { Percent change } \\
\text { from regional } \\
\text { standard } \\
\text { deviation to } \\
\text { standard error } \\
\text { of estimate }\end{array}$ & $\begin{array}{c}\text { No. of } \\
\text { stations in } \\
\text { analysis for } \\
\text { equation }\end{array}$ \\
\hline \multicolumn{9}{|c|}{ Northeast region } \\
\hline Mean & $\mu=135.8 \mathrm{~A}^{0.631} \mathrm{SH}^{-0.0911} \mathrm{SL}^{0.370}$ & $3.87(0.578) \log _{10}$ & A: $0.20-38,600 \mathrm{mi}^{2}$ & 0.884 & 50 & $0.204 \log _{10}$ & -64.7 & 99 \\
\hline $\mathrm{L}-\mathrm{CV}$ & $\tau_{2}=\log _{10}\left(2.979 \mathrm{~A}^{-0.0168}\right)$ & $.434(0.0949)$ & SH: 0.182-39.9 & .034 & 21 & .0885 & -6.74 & 99 \\
\hline L-skew & $\tau_{3}=0.315$ & $.315(0.153)$ & SL: $1.48-65.0 \mathrm{ft} / \mathrm{mi}$ & -- & -- & -- & -- & 99 \\
\hline L-kurtosis & $\tau_{4}=0.203$ & $.203(0.143)$ & & -- & -- & -- & -- & 99 \\
\hline \multicolumn{9}{|c|}{ Southeast region } \\
\hline Mean & $\mu=182.7 \mathrm{~A}^{0.654} \mathrm{SH}^{-0.299} \mathrm{SL}^{0.339}$ & $3.90(0.497) \log _{10}$ & A: $1.48-35,400 \mathrm{mi}^{2}$ & .845 & 44 & $.181 \log _{10}$ & -63.6 & 92 \\
\hline $\mathrm{L}-\mathrm{CV}$ & $\tau_{2}=\log _{10}\left(1.359 \mathrm{~A}^{0.0685} \mathrm{SH}^{-0.107} \mathrm{SL}^{0.336}\right)$ & $.462(0.140)$ & SH: $1.67-41.2$ & .421 & 23 & .100 & -28.6 & 92 \\
\hline L-skew & $\tau_{3}=\log _{10}\left(1.229 \mathrm{~A}^{0.0395} \mathrm{SL}^{0.262}\right)$ & $.374(0.184)$ & $\mathrm{SL}: 1.22-70.0 \mathrm{ft} / \mathrm{mi}$ & .149 & 37 & .154 & -16.3 & 92 \\
\hline L-kurtosis & $\tau_{4}=0.249$ & $.249(0.173)$ & & - & -- & - & - & 92 \\
\hline \multicolumn{9}{|l|}{ West region } \\
\hline Mean & $\mu=104.7 \mathrm{~A}^{0.582} \mathrm{SH}^{-0.143} \mathrm{SL}^{0.425}$ & $3.90(0.522) \log _{10}$ & A: $2.45-35,200 \mathrm{mi}^{2}$ & .547 & 85 & $.321 \log _{10}$ & -38.5 & 176 \\
\hline $\mathrm{L}-\mathrm{CV}$ & $\tau_{2}=\log _{10}\left(5.488 \mathrm{~A}^{-0.0218} \mathrm{SH}^{-0.124}\right)$ & $.551(0.146)$ & SH: $1.53-84.9$ & .090 & 31 & .131 & -10.3 & 176 \\
\hline L-skew & $\tau_{3}=\log _{10}\left(3.530 \mathrm{SH}^{-0.109}\right)$ & $.424(0.192)$ & $\mathrm{SL}: 3.52-130 \mathrm{ft} / \mathrm{mi}$ & .031 & 40 & .166 & -13.5 & 176 \\
\hline L-kurtosis & $\tau_{4}=0.262$ & $.262(0.175)$ & & -- & -- & -- & -- & 176 \\
\hline
\end{tabular}

${ }^{1}$ Adjusted R-squared presented so equations based on different numbers of stations and using different independent variables—contributing drainage area, basin shape factor, and stream slope—can be compared. 